\title{
Reconstitution of SNARE Mediated Membrane Fusion
}

\section{Alex John Boudreau Kreutzberger}

B.S. Chemistry, University of North Carolina at Wilmington, 2011

\section{A dissertation presented to the Graduate Faculty of the University of Virginia} in Candidacy for the Degree of Doctor of Philosophy

\section{Department of Molecular Physiology and Biological Physics}

Center for Membrane and Cell Physiology

University of Virginia

December 2016

Lukas K. Tamm

J. David Castle

David Cafiso

Chris Stroupe 


\section{Abstract}

Regulated exocytosis is a process by which cells release neurotransmitters, peptides, proteins, and small molecules in response to a stimulus. This process is necessary for cell-cell communication in multi-cellular organisms. The final step of regulated exocytosis is membrane fusion, where the membrane of a secretory vesicle merges with the plasma membrane, opening a pore releasing soluble cargo. The molecular machinery that controls fusion is coupled to respond to intracellular calcium. The proteins involved include the SNARE proteins (the fusion machinery), the calcium sensor synaptotagmin, and the regulatory proteins complexin, Munc18, Munc13, and CAPS.

I will show the use of planar supported bilayers as a model system to study membrane fusion of single vesicles (Chapter 3). This assay was used to investigate the activity of different plasma membrane target SNARE complexes (Chapter 4), the effect of membrane curvature (Chapter 5) and cholesterol (Chapter 6) on the fusion process, how complexin-1 interacts with the plasma membrane SNARE proteins (Chapter 7), and how the fusion process is coupled to calcium (Chapter 8). These results have led to a new model of membrane fusion. In the absence of calcium, secretory vesicle fusion is arrested in the presence of the SNARE proteins, Munc18, and complexin, but will fuse readily in the presence of calcium. Meanwhile, the protein CAPS or Munc13 act as a kinetic factors controlling the rate of fusion in response to a calcium stimulus. 


\section{Table of Contents}

$\begin{array}{ll}\text { Abstract } & \text { i }\end{array}$

$\begin{array}{ll}\text { Table of Contents } & \text { ii }\end{array}$

Acknowledgments $\quad$ V

$\begin{array}{ll}\text { Chapter 1: Introduction } & 1\end{array}$

1.1 Membrane fusion $\quad 2$

$\begin{array}{ll}1.2 \text { Regulated exocytosis } & 3\end{array}$

$\begin{array}{ll}1.3 \text { The SNARE complex } & 4\end{array}$

Chapter 2: Materials and Methods

2.1 Protein expression and purification $\quad 8$

2.2 Proteoliposome preparations 11

2.3 Formation of planar supported bilayers 11

2.4 Co-floatation assay $\quad 13$

2.5 Plasmids and shRNA constructs 13

$\begin{array}{ll}2.6 \text { Cell culture } & 16\end{array}$

$\begin{array}{ll}2.7 \text { DCV purification } & 17\end{array}$

2.8 Total internal reflection fluorescence microscopy 18

2.9 Single proteoliposome fusion assay $\quad 19$

$\begin{array}{ll}2.10 \text { Proteoliposome docking assay } & 20\end{array}$

2.11 Single DCV docking and fusion assay $\quad 20$

2.12 Single DCV Ca ${ }^{2+}$ triggered assay 22

2.13 Western blotting $\quad 22$

2.14 Ensemble lipid mixing assay $\quad 22$ 
2.15 Cryo-electron microscopy 23

$\begin{array}{ll}2.16 \text { Phosphate assay } & 23\end{array}$

$\begin{array}{ll}2.17 \text { Protein assay } & 23\end{array}$

$\begin{array}{ll}2.18 \text { EPR measurements } & 24\end{array}$

$\begin{array}{ll}2.19 \text { Fluoresce anisotropy measurements } & 26\end{array}$

$\begin{array}{ll}2.20 \text { NMR spectroscopy } & 26\end{array}$

Chapter 3: Single Vesicle Fusion with Planar Supported Bilayers

$\begin{array}{ll}3.1 \text { Summary } & 28\end{array}$

3.2 Introduction $\quad 29$

3.3 Formation of planar supported bilayers $\quad 30$

3.4 SNARE-mediated single vesicle fusion 31

3.5 Single vesicle fusion with physiological vesicles 39

$\begin{array}{ll}\text { 3.6 Discussion } & 46\end{array}$

Chapter 4: Assembly and comparison of plasma membrane SNARE complexes

$\begin{array}{ll}\text { 4.1 Summary } & 47\end{array}$

$\begin{array}{ll}4.2 \text { Introduction } & 48\end{array}$

$\begin{array}{ll}4.3 \text { Results } & 50\end{array}$

4.4 Discussion $\quad 57$

Chapter 5: The role of membrane curvature and SNARE mediated fusion

$\begin{array}{ll}5.1 \text { Summary } & 59\end{array}$

$\begin{array}{ll}5.2 \text { Introduction } & 60\end{array}$

$\begin{array}{ll}5.3 \text { Results } & 61\end{array}$

$\begin{array}{ll}5.4 \text { Discussion } & 83\end{array}$ 
Chapter 6: High cholesterol obviates a prolonged hemifusion intermediate in SNAREmediated membrane fusion

$\begin{array}{lr}\text { 6.1 Summary } & 88\end{array}$

$\begin{array}{lr}\text { 6.2 Introduction } & 89\end{array}$

$\begin{array}{ll}6.3 \text { Results } & 92\end{array}$

$\begin{array}{ll}\text { 6.4 Discussion } & 104\end{array}$

Chapter 7: Complexin-1 interaction with plasma membrane SNAREs

$\begin{array}{ll}\text { 7.1 Summary } & 109\end{array}$

$\begin{array}{ll}7.2 \text { Introduction } & 110\end{array}$

$\begin{array}{ll}7.3 \text { Results } & 116\end{array}$

$\begin{array}{ll}\text { 7.4 Discussion } & 141\end{array}$

Chapter 8: Reconstitution of calcium dependent fusion

$\begin{array}{ll}8.1 \text { Summary } & 146\end{array}$

$\begin{array}{ll}8.2 \text { Introduction } & 147\end{array}$

$\begin{array}{ll}8.3 \text { Results } & 148\end{array}$

$\begin{array}{ll}\text { 8.4 Discussion } & 177\end{array}$

Chapter 9: Conclusions

9.1 Summary and Outlook 182

Appendix

A.1 Membrane labeling of physiological vesicles 184

A.2 Asymmetric PE Distribution Controls Fusion Pore Lifetime and Probability 190

A.3 Examining fusion pores with electron microscopy 195

A.4 Imaging whole cell exocytosis by fluorescence microscopy 199

$\begin{array}{ll}\text { References } & 202\end{array}$ 


\section{Acknowledgments}

I would like to thank Dr. Lukas Tamm and Dr. David Castle for providing me with the opportunity to conduct research in their laboratories. Dr. David Cafiso and Dr. Chris Stroupe for being on my committee and always being willing to engage me in scientific discussion and provide guidance and time as I conducted my dissertation research. I would also like to thank my family and many friends who have supported me as I have moved through my scientific career. I would also like to especially thank Volker Kiessling and Binyong Liang for assisting me in my research throughout my graduate work. 


\section{Chapter 1: Introduction}

Regulated exocytosis is a highly-coordinated process where specialized secretory cells release soluble contents in response to an external stimulus. Highly studied examples of exocytosis include the release of neurotransmitters from neurons, secretion of epinephrine, norepinephrine and dopamine from chromaffin cells, and the release of insulin from $\beta$-cells (Hanson, et al., 1997; Bader, et al., 2002; Thorn, et al., 2016) Common principles exist between all exocytocytotic secretion events. Secretory contents are stored in vesicles such as synaptic vesicles or large dense core vesicles also commonly referred to as granules (Gondre-Lewis, et al., 2012). These secretory vesicles are targeted to the plasma membrane, where they are stimulated by calcium to release their contents (Gustavsson, et al., 2012). The release of secretory contents occurs via membrane fusion, where the secretory vesicle membrane merges with the plasma membrane and release cargo through a fusion pore (Vardjahn, et al., 2013). The SNARE proteins drive the fusion process while the protein synaptotagmin is responsible for the calcium response. Other proteins (such as Munc18, Munc13, and complexin) are involved in regulating this fusion process in a manner that couples it with calcium (Söllner, 2003; Jahn \& Fasshauer, 2012; Südhof, 2013), while the composition of the vesicle and plasma membrane are also thought to contribute to the fusion process (Martin, 2012; Chasserot-Golaz, et al., 2010). In this thesis, the use of planar supported bilayers will be disccused as a tool to investigate membrane fusion with a specific emphasis on using this system to investigate how calcium is coupled to the regulated exocytosis machinery and what role membrane lipid composition plays in this process. 


\subsection{Membrane Fusion}

The merging of two separate lipid bilayers into one is known as membrane fusion, which is a fundamental biological process that allows membrane components and soluble contents of cell compartments to exchange. Processes such as exocytosis, membrane trafficking, viral entry, and fertilization are dependent on a membrane fusion event. Biologically relevant membrane fusion is catalyzed by fusion proteins that pull the membranes together providing energy in the form of a mechanical force. The two most studied classes of fusion proteins are the SNARE proteins and viral envelope fusion proteins. The first function in intracellular trafficking and exocytosis and the latter facilitate viral infection (Martens \& McMahon, 2008; Tamm, et al., 2003).

All membrane fusion goes through similar steps and intermediates leading to the formation of a fusion pore. These intermediates include tethering and docking of the two membranes to one another, initiation of fusion where a point like protrusion occurs in one of the bilayers, hemifusion stalk formation which may progress into an extended hemifusion diaphragm and then to a fusion pore, Figure 1.1 (Martens \& McMahon, 2008; Chernomordik \& Kozlov, 2008). In vitro fusion assays also appear to have a dead end off pathway stable hemifusion intermediate that does not lead to the opening of a fusion pore (Diao, et al., 2012; Kreutzberger, et al., 2015; Chlanda, et al., 2016). 


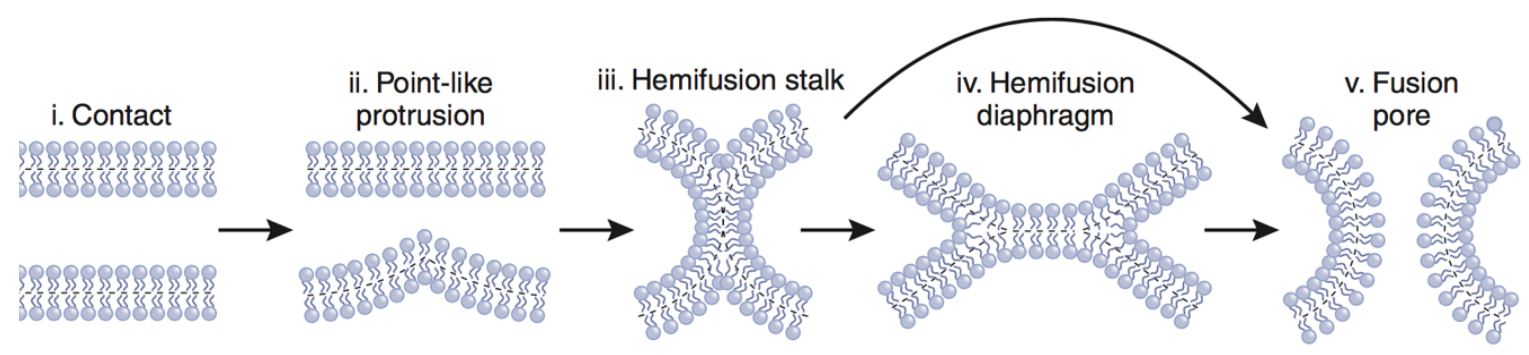

Figure 1.1: Fusion-through hemifusion pathway of lipid bilayer fusion. (i) Pre-fusion contact. (ii) A point-like membrane protrusion minimizes the energy of the hydration repulsion between the proximal leaflets of the membranes coming into immediate contact. (iii) A hemifusion stalk with proximal leaflets fused and distal leaflets unfused. (iv) Optional stalk expansion yields the hemifusion diaphragm. (v) A fusion pore forms either in the hemifusion diaphragm bilayer or directly from the stalk. Dashed lines show the boundaries of the hydrophobic surfaces of monolayers. Figure is adapted from (Chernomordik \& Kozlov, 2008).

\subsection{Regulated exocytosis}

Specialized secretory cells release soluble contents in response to external stimulus that is essential for cell-cell communication in multi-cellular organisms (Jahn \& Fasshauer, 2012; Südhof, 2013). The most rapid and highly coordinated form of regulated exocytosis occurs in neurons. A similar mechanism occurs in neuroendocrine cells (such as chromaffin cells) and endocrine cells (such as $\beta$-cells) but with a slower response time to calcium than that observed for neurons (Martin, 2003).

Neurons, neuroendocrine cells, and endocrine cells are all electrically excitable cells that transmit signals through electrical and chemical signals (Hodgkin, et al., 1952; Dean \& Matthews, 1968; Neher \& Marty, 1982). Ion pumps and channels in the cells maintain chemical 
gradients of sodium, potassium, chloride, and calcium that create a voltage gradient across the membrane. Depolarization of this voltage gradient leads to the opening of calcium channels and the triggering of exocytosis (Katz, et al., 1982; Zbili, et al., 2016).

A similar core protein machinery drives exocytosis in all three cell types. The SNARE proteins provide the energy for membrane fusion, while other proteins regulate the SNARE proteins function and couple their fusion to stimulation by calcium (Jahn \& Fasshauer, 2012; Südhof, 2013). The calcium sensor synaptotagmin binds anionic membranes after binding calcium. Munc18 is an essential protein that regulates syntaxin-1a by opening or closing its regulatory domain. Complexin-1 is a small $\alpha$-helical protein the inhibits spontaneous fusion and coordinates a synchronized response in neurons. There are also a set of proteins that prime exocytosis to occur in a kinetically fast and coordinated manner in response to calcium. These proteins include Munc13-1, Munc13-2, Munc13-4, and CAPS-1 and CAPS-2 (Hanson, et al., 1997; Jahn \& Fasshauer, 2012; Jahn \& Scheller, 2006; James \& Martin, 2013; Südhof, 2013).

\subsection{SNARE Complex}

SNARE proteins are transmembrane or lipid anchored membrane proteins that contain an $\alpha$ helical SNARE motif of 60-70 amino acids arranged in heptad repeats (Fasshauer, 2003). These proteins occur in sets of four corresponding SNARE motifs with at least one protein present the lipid bilayer of each membrane undergoing fusion (Tamm, et al., 2003; Jahn \& Fasshauer, 2012). A SNARE motif is an $\alpha$-helix forming sequence which assembles into an extended coiled-coil 
when 4 SNARE motifs come together (Sutton, et al., 1998). The central layer of the assembled SNARE complex contains SNARE domains where 3 helixes contain a glutamine (termed Qa, Qb, and Qc SNAREs) and the fourth helix contains an arginine (termed R SNARE). These 4 residues serve as a template to align the SNARE motifs (Fasshauer, et al., 1998; Kloepper, et al., 2007). The assembly of the SNAREs into the 4 helical bundle releases free energy ( $\sim 36 \mathrm{kBT})$ that provides a mechanical force to drive the fusion of the two membranes (Fasshauer, et al., 2002; Gao, et al., 2012).

The SNARE proteins for exocytosis are the most frequently studied SNARE complex were there are three proteins possessing the 4 SNARE motifs. The SNARE proteins syntaxin-1a and SNAP-25 are present in the plasma membrane (frequently noted as target (t-) SNAREs) and synaptobrevin-2 is in the secretory vesicle membrane (frequently noted as vesicle (v-) SNARE) (Jahn \& Scheller, 2006; Jahn \& Fasshauer, 2012). In syntaxin-1a (Qa SNARE) and synaptobrevin-2 (R SNARE) (as well as most other SNAREs) the SNARE motifs are connected by a short linker to a C-terminal transmembrane region. However, SNAP-25 possesses two SNARE motifs ( $\mathrm{Qb}$ and $\mathrm{Qc})$ and that are connected by a linker that is palmitoylated, which anchors it in the plasma membrane (Jahn \& Scheller, 2006; Jahn \& Fasshauer, 2012). Syntaxin1a also possess an N-terminal regulatory domain that contains an antiparallel three-helix bundle (termed the Habc domain) that is connected to the SNARE domain by a flexible linker (Fernandez, et al., 1998; Lerman, et al., 2000). Upon contact, the SNARE motifs begin to assemble at the N-terminal ends in trans with syntaxin-1a and SNAP-25 anchored in the plasma membrane and synaptobrevin- 2 anchored in the secretory vesicle membrane forming the tight SNARE bundle (Sutton, et al., 1998; Stein, et al., 2009). They then zipper in the N- to C-terminal 
direction (Hanson, et al., 1997; Pobbati, et al., 2006). This assembly releases the energy necessary for fusion to occur (Li, et al., 2007; Wiederhold \& Fasshauer, 2009; Gao, et al., 2012).

After assembly of the SNARE complex and membrane fusion, the SNARE complex is disassembled by the AAA+ATPase NSF and the protein $\alpha$-SNAP (Ryu, et al., 2016). Four $\alpha$ SNAP molecules bind to one SNARE complex, which is then bound by NSF in the ATP bound state which undergoes one round of ATP hydrolysis of six ATP molecules to disassemble the SNARE complex in spring loaded mechanism (Zhao, et al., 2015; Ryu, et al., 2015). This disassembly of the complex allows the SNAREs to be used in repeated cycles of fusion followed by disassembly (Jahn \& Scheller, 2006).

SNARE proteins are the minimum requirement to drive fusion in vitro (Weber, et al., 1998), but other proteins in the whole machinery are necessary to couple fusion to respond to calcium in a coordinated manner (Jahn \& Fasshauer, 2012; Sudhof, 2014; Sudhof \& Rothman, 2009). In the work presented here a minimal (SNARE only) single particle fusion assay will be described. Then, a description of incorporating physiological vesicles with the molecular machinery necessary to respond to calcium will be shown, which then allows the incorporation of other proteins necessary to regulate fusion to only occur in the presence of calcium. 


\section{Chapter 2: Materials and Methods}

The following materials were purchased and used without further purification:

Porcine brain L- $\alpha$-phosphatidylcholine (bPC), porcine brain L- $\alpha$-phosphatidylethanolamine (bPE), porcine brain L- $\alpha$-phosphatidylserine (bPS), 1,2-dioleoyl-sn-glycero-3-

phosphoethanolamine-N-(lissamine rhodamine B sulfonyl) (Rh-DOPE), and 1-palmitoyl-2oleoylphosphatidylcholine (POPC), 1-palmitoyl-2-oleoyl-phosphatidylserine (POPS) (Avanti Polar Lipids, Alabaster, Al).

1,1'-dioctadecyl-3,3,3',3'-tetramethyl-indodicarbocyanine perchlorate (DiD) and sulforhodamine b (Life Technologies, Frederick, MD).

Cholesterol, octyl- $\beta$-D-glucopyranoside ( $\beta O G)$, sodium cholate, 2,2',2",2"'-(ethane-1,2diyldinitrilo)tetra acetic acid (EDTA), and glycerol (Sigma, St Louis, MO).

3-[(3-cholamidopropyl)dimethylammonio]-1-propanesulfonate (CHAPS) (Anatrace, Maumee, $\mathrm{OH})$. 
2-[4-(2-hydroxyethyl)piperazin-1-yl]ethanesulfonic acid (HEPES), potassium chloride (KCl) (Research Products International, MountProspect, Il).

Chloroform, ethanol, Contrad detergent, all inorganic acids, bases, and hydrogen peroxide (Fisher Scientific, Fair Lawn, NJ).

Water was purified first with deionizing and organic-free 4filters(Virginia Water Systems, Richmond, VA) and then with a NANOpure system (Barnstead, Dubuque, Ia).

\subsection{Protein expression and purification}

Protein Expression and Purification

Synaptobrevin-2, syntaxin-1a, SNAP-25, Munc18, and complexin-1 from Rattus norvegicus in pET28a vector were expressed in Escherichia coli (E. coli) and purified as described in (Fasshauer, et al., 2002; Fasshauer \& Margittai, 2004). Constructs of synaptobrevin-2 included residues 49-96, 1-96, and 1-117. The cysteine-free variant of SNAP-25A consisting of residues 1-206 was used for preparing the $\Delta \mathrm{N}$ and binary complex. Syntaxin-1a constructs consisted of

residues 183-288 and 1-288. Synaptobrevin-2 and SNAP-25 were purified by $\mathrm{Ni}^{2+}$-NTA affinity chromatography followed by ion exchange chromatography using a MonoQ or MonoS columns in the presence of $15 \mathrm{mM}$ CHAPS. Syntaxin-1a for the $\Delta \mathrm{N}$ complex was purified in CHAPS and in DPC for the binary complex. Munc13 expression was carried out in BL21(DE3) cells by IPTG 
induction. Harvested cell pellets were re-suspended then lysed in the extraction buffer $(20 \mathrm{mM}$ HEPES, pH7.4, $500 \mathrm{mM} \mathrm{NaCl}, 8 \mathrm{mM}$ imidazole). Supernatant of lysed cells was collected and subjected to binding of Ni-NTA beads. After extensive wash of protein-bound Ni-NTA beads with the wash buffer (20 mM HEPES, pH7.4, $500 \mathrm{mM} \mathrm{NaCl}, 20 \mathrm{mM}$ imidazole, 10\% glycerol), Munc13 was eluted from Ni-NTA with elution buffer (20 mM HEPES, pH 7.4, $400 \mathrm{mM}$ imidazole). After the Histag cleavage by bovine thrombin, Munc13 was further purified by subsequent MonoQ ion-exchange chromatography. Purities of all proteins were verified by SDSPAGE.

Syntaxin-1a(183-288):SNAP-25:Synaptobrevin-2 (49-96)(1:1:1): The " $\Delta N$ complex"

The $\Delta \mathrm{N}$ complex was prepared by one of two procedures. The first was purifying all the proteins separately and mix them 1:1:1 in the presence of CHAPS, then purify the complex on a MonoQ ion exchange column. The second procedure was using the pET28a vector for SNAP-25A and the pETDuet-1 vector for syntaxin-1a (183-288) and synaptobrevin-2 (49-96) (Stein, et al., 2007).

\section{Monomeric syntaxin-1a}

Monomeric syntaxin-1a (either 183-288 or 1-288) was purified as described above but in the presence of DPC detergent which keeps syntaxin-1a strictly monomeric 
Syntaxin-1a:SNAP-25 (1:1): The "binary complex"

Monomeric syntaxin-1a and SNAP-25 are mixed 1-1 in the presence of DPC overnight then purified on a MonoQ ion exchange column.

\section{Wild-type SNAP-25 alkylation with dodecyl chains}

Following purification on a Ni-NTA column and cleavage of the histidine tag, wild-type SNAP25a was diluted with $20 \mathrm{mM}$ HEPES, $\mathrm{pH} 7.1,500 \mathrm{mM} \mathrm{NaCl}, 0.1 \%$ DPC and concentrated to remove excess imidazole and to introduce DPC detergent. A 20-fold molar excess (over Cys, 80fold over protein) of DTT was added for 2 hours at room temperature followed by desalting on a PD10 column (GE Healthcare). The fractions containing SNAP-25A were incubated overnight with a 10-fold molar excess (over Cys, 40-fold over protein) of DdMTS, which was added from a $250 \mathrm{mM}$ stock solution in acetonitrile. The resulting d-SNAP-25a sample was purified on a MonoQ column in the presence of $0.1 \%$ DPC. Samples were analyzed by MALDI-TOF mass spectrometry for the number of added dodecyl chains. MALDI-TOF was performed in the W.M. Keck Biomolecular Mass Spectrometry Laboratory at the University of Virginia School of Medicine. 


\subsection{Proteoliposome preparations}

\section{Content- and lipid-labeled v-SNARE proteoliposomes}

Synaptobrevin-2 proteoliposomes containing $100 \mathrm{mM}$ sulforhodamine B and lipid compositions as indicated in the text. The desired lipids mixed in organic solvents were evaporated under a stream of $\mathrm{N}_{2}$ gas and placed under vacuum for 1 hour. The dried lipid films were dissolved with $118 \mu \mathrm{L}$ of $110 \mathrm{mM} \beta \mathrm{OG}$ in reaction buffer (20 mM HEPES, $150 \mathrm{mM} \mathrm{KCl,} \mathrm{pH} \mathrm{7.4)} \mathrm{containing}$ $100 \mathrm{mM}$ sulforhodamine followed by the addition of appropriate volumes of synaptobrevin-2 to reach final volumes of $\sim 180 \mu \mathrm{L}$ and the desired lipid-to-protein ratio of 400 . After 1 hour of equilibration, the mixture was diluted to a final volume of $550 \mu \mathrm{L}$ and a $\beta \mathrm{OG}$ concentration of $\sim 24 \mathrm{mM}$ with buffer containing $100 \mathrm{mM}$ sulforhodamine B. This lipid/detergent/protein mixture was then loaded on a G-50 superfine Sephadex (GE Healthcare, Piscataway, NJ) column to remove free detergent and the labeled proteoliposomes were collected and used within 48 hours. For non-content labeled liposomes sulforhodamine B dye was not included in buffers and dialysis was used to remove the free detergent instead of column.

\section{Proteoliposome reconstitution with plasma membrane SNARE proteins}

Proteoliposomes with $\Delta \mathrm{N}$ complex, the binary complex, or monomeric syntaxin-1a and dodecylSNAP-25 were formed by rapid dilution and dialysis of sodium cholate from the respective proteins in detergent and lipid indicated in text (Domanska, et al., 2009; Wagner \& Tamm, 2001). The lipid to protein rations are indicated in the text. Lipids were mixed in chloroform and 
dried on the bottom of glass text tubes under a stream of nitrogen. The dried lipid films were dissolved with sodium cholate in reaction buffer followed by addition of the appropriate concentrations of protein and detergent-free buffer to obtain a solution of $25 \mathrm{mM}$ sodium chelated in a final volume of $180 \mu \mathrm{L}$. The lipid, protein, and detergent mixtures were equilibrated at room temperature for 1 hour and then diluted by addition of reaction buffer to a final volume of $550 \mu \mathrm{L}$ to a concentration below the critical micellar concentration. Samples were then dialyzed overnight against $500 \mathrm{~mL}$ of reaction buffer at $4^{\circ} \mathrm{C}$ with one buffer change.

\subsection{Formation of planar supported bilayers}

Planar supported bilayers with reconstituted plasma membrane SNARE proteins were prepared by the Langmuir-Blodgett/vesicle fusion technique as described in previous studies (Domanska, et al., 2009; Wagner \& Tamm, 2001; Kalb, et al., 1992). Quartz slides were cleaned by boiling in Contrad detergent for 10 minutes, then is placed in a bath-sonicator for 20 minutes, and rinsing thoroughly with deionized water. The slides were then cleaned by dipping 3:1 sulfuric acid: hydrogen peroxide for 10 minutes then rinsed thoroughly in water. The first leaflet of the bilayer was prepared by Langmuir-Blodgett transfer directly onto the quartz slide. A lipid monolayer of the desired lipid composition as indicated in the text was prepared on a pure water surface in a Nima 611 Langmuir-Blodgett trough (Nima, Conventry, UK) by applying the lipid mixture from a chloroform solution. After allowing the solvent to evaporate for 10 minutes, the monolayer was compressed at a rate of $10 \mathrm{~cm}^{2} /$ minute to reach a surface pressure of $32 \mathrm{mN} / \mathrm{m}$. After equilibration for 5 to 10 minutes, a clean quartz slide was rapidly $(200 \mathrm{~mm} / \mathrm{min})$ dipped into the trough and slowly $(5 \mathrm{~mm} / \mathrm{min})$ withdrawn, while a computer maintained a constant surface 
pressure and monitored the transfer of lipids with head groups down onto the hydrophilic substrate. Proteoliposomes containing plasma membrane SNARE proteins ( $77 \mu \mathrm{M}$ total lipid in $1.3 \mathrm{~mL}$ ) were added and incubated at room temperature for 2 hour to introduce the protein complex and form the second leaflet of the supported bilayer. Excess unfused proteoliposomes were then removed by perfusion with $10 \mathrm{~mL}$ of reaction buffer with EDTA or calcium as indicated in the text. If not noted $100 \mu \mathrm{M}$ EDTA was used in all buffers not containing calcium.

\subsection{Co-floatation assay}

The efficiency of protein insertion into liposomes was checked by a procedure similar to that described in (Hernandez, et al., 2012). Liposomes containing plasma membrane SNARE (50 $\mu \mathrm{L})$ were mixed with $80 \%$ Nicene (Axis Shield, Dundee, Scotland) in a $250 \mu \mathrm{L}$ centrifuge insert to make a $40 \%$ Nycodenz solution. The $50 \mu \mathrm{L}$ of $30 \%$ Nycodenz was layered onto the $40 \%$ Nycodenz layer followed by a layer of $50 \mu \mathrm{L}$ reaction buffer on top. The density gradient was centrifuged for 1.5 hours in a Beckman TL-100 ultracentrifuge with a TLS55 rotor at 197,000 g and $4^{\circ} \mathrm{C}$. Upon completion, $20 \mu \mathrm{L}$ aliquots were carefully taken from the top of the gradient and western blots were used to detect the protein.

\subsection{Plasmids and shRNA constructs}


pEGFP-N1-NPY (a kind gift from Wolf Almers) was used to sub-clone NPY into a pmCherryN1 and a pmRuby vector. Results from NPY-mRuby were slightly brighter in our experimental setup and used in all subsequent experiments, but initial experiments revealed no differences in results obtained with NPY-mCherry or NPY-mRuby.

For simultaneous shRNA knockdown of multiple synaptotagmin isoforms, we created a modular vector platform similar to (Xu, et al., 2009) based on pLKO.5. pLKO.5 (Sigma-Aldrich) was digested with EcoRI and PpuMI, blunted with T4 DNA polymerase, and re-circularized. To obtain the empty vector plasmid pLKO.P-emtpy, EcoRI and BstZ17I sites were introduced by site-directed mutagenesis (Zheng, et al., 2004) using primer pair 1\&2 (Table 2.1). shRNA expression cassettes targeting syt1 (TRCN0000093258) and syt9 (TRCN0000379591) were amplified by PCR from Mission shRNA plasmids (Sigma-Aldrich) using primer pairs $3 \& 5$ or 4\&5, respectively, and subsequently digested with MfeI and BstZ17I. These digested fragments were sequentially ligated into pLKO.P-empty digested with EcoRI and BstZ17I, eventually yielding the double knockdown plasmid pLKO.P-syt9-syt1.

For the CAPS1 knockdown, oligonucleotide 6 (Kabachinski, et al., 2016) was assembled into pLKO.5 digested with KpnI and EcoRI using the NEBuilder HiFi DNA Assembly reaction (NEB). 
In order to express shRNA-resistant syt1, pHluorin was first removed from pCI-pHluorin-syt1 (a kind gift of Arun Anantharam described in (Rao, et al., 2004) by overlap extension PCR cloning (van den Ent \& Lowe, 2006) using primer pair 7\&8. Subsequently, five silent mutations were introduced into the shRNA target region of syt1 by site-directed mutagenesis using primer pair $9 \& 10$.

For the CAPS1 rescue, pmKate2-resCAPS1 containing eight silent mutations in the shRNA target site (a kind gift of Thomas Martin described in (Kabachinski, et al., 2016)was digested with BamHI and NotI, blunted with T4 DNA polymerase and re-circularized in order to remove mKate2.

All plasmid sequences were verified by Sanger DNA sequencing (Genewiz).

\begin{tabular}{|c|c|c|}
\hline \# & Name & Sequence $\left(5^{\prime}->3^{\prime}\right)$ \\
\hline 1 & ins_BstZ17I_fw & AGATCTTGAGACAAATGGCAGTATACATCCACAATTTTAAAAG \\
\hline 2 & ins_EcoRI_ry & TGCCATTTGTCTCAAGATCTAGAATTCTCOCCTOGGGGTTG \\
\hline 3 & TRC1.5_shRNA_Mfel_fw & TATTCAATTGTTCACCGAGGGCCTATTTC \\
\hline 4 & TRC2_shRNA_Mfel_f $w$ & TATT CAATTGGGCCTATTTCOCATGATTCC \\
\hline 5 & TRC_shRNA_BstZ17I_rv & AGCTAAGTATACGGATGAATACTGCCATTTGTC \\
\hline 6 & CAPS1_shRNA & $\begin{array}{l}\text { TTGTGGAAAGGACGAGGTACCGGTCGTCTTCTTCATCTTOCTOGTCACT } \\
\text { GTGAAGCTTGATAGTGATGAGGAGGATGAGGAAGACGACTATTTTTTG } \\
\text { AATTCTAGATCTTGAGACAAATGG }\end{array}$ \\
\hline 7 & syt1_del_pHluorin_fw & СTACTCTTGTGCCAGGGTGTGGTCTCCTCAGGCGGAAGCGGAGGC \\
\hline 8 & syt1_del_pHluorin_rv & CTTCTTGACAGCCAGCATGGCATC \\
\hline 9 & syt1_shRNA-resist_fw & TCGAACAGATTCAGAAGGTGCAGGTGGTGGTAACTG \\
\hline 10 & syt1_shRNA-resist_rv & ACCACCTGCACCTTCTGAATCT GTTCGAACGGAACTTC \\
\hline
\end{tabular}

Table 2.1: Table of primers described in plasmids section of Materials and Methods. Mismatches/mutations are highlighted in bold and restriction sites are underlined. 


\subsection{Cell culture}

Pheochromocytoma cells (PC12) were cultured on $10 \mathrm{~cm}$ plastic cell culture plates at $37^{\circ} \mathrm{C}$ in $10 \% \mathrm{CO}_{2}$ in Dulbecco's Modified Eagle Medium (DMEM) High Glucose 1 X Gibco supplemented with 10\% horse serum (Cellgro), 10\% calf serum $\left(\mathrm{Fe}^{+}\right)$(Hyclone), and 1\% penicillin/streptomycin mix. Medium was changed every 2-3 days and cells were passed after reaching $90 \%$ confluency by incubating $5 \mathrm{~min}$ in HBSS and re-plating in fresh medium. Cells were transfected by electroporation using an Electro Square Porator ECM 830 (BTX). After harvesting and sedimentation, cells were suspended in a small volume of sterile cytomix electroporation buffer (van den Hoff, et al., 1992) (120 mM KCl, $10 \mathrm{mM} \mathrm{KH}_{2} \mathrm{PO}_{4}, 0.15 \mathrm{mM}$ $\mathrm{CaCl}_{2}, 2 \mathrm{mM}$ EGTA, $25 \mathrm{mM}$ HEPES-KOH, $5 \mathrm{mM} \mathrm{MgCl}_{2}, 2 \mathrm{mM}$ ATP, and $5 \mathrm{mM}$ glutathione, $\mathrm{pH}$ 7.6) and then counted and diluted to $\sim 14 \times 10^{6}$ cells $/ \mathrm{mL} .700 \mu \mathrm{L}$ of cell suspension $\left(\sim 10 \times 10^{6}\right.$ cells) and $30 \mu \mathrm{g}$ of DNA were placed in an electroporation cuvette with $4 \mathrm{~mm}$ gap and two $255 \mathrm{~V}, 8 \mathrm{mms}$ electroporation pulses were applied. Cells were then transferred to a $10 \mathrm{~cm}$ cell culture dish with $10 \mathrm{~mL}$ of normal growth medium. NPY-Ruby transfected cells were cultured under normal conditions for 3 days after transfection and then used for fractionation. Transient knockdown cell lines were generated by transfecting shRNA as described above and maintaining cells under $2 \mu \mathrm{g} / \mathrm{mL}$ puromycin selection beginning 24 hours after transfection. Cells were later transfected with NPY-Ruby and, when relevant, shRNA resistant plasmids 3 days prior to fractionation. 


\subsection{DCV purification}

DCVs were purified using iso-osmotic media as follows. PC12 cells (15-30 10-cm plates depending on experiments) were scraped into PBS, pelleted by centrifugation, re-suspended and washed once in homogenization medium (0.26 M sucrose, $5 \mathrm{mM}$ MOPS, and $0.2 \mathrm{mM}$ EDTA). Following resuspension in $(3 \mathrm{ml})$ homogenization medium containing protease inhibitor (Roche Diagnostics), the cells were cracked open using a ball bearing homogenizer with a 0.2507 -inch bore and 0.2496-inch diameter ball. The homogenate was then spun at $4000 \mathrm{rpm}(1000 \mathrm{xg}), 10$ min at $4^{\circ} \mathrm{C}$ in fixed-angle micro centrifuge to pellet nuclei and larger debris. The post nuclear supernatant (PNS) was collected and spun at $11,000 \mathrm{rpm}(8000 \mathrm{x} \mathrm{g}), 15 \mathrm{~min}$ at $4^{\circ} \mathrm{C}$ to pellet mitochondria. The post mitochondrial supernatant (PMS) was then collected, adjusted to $5 \mathrm{mM}$ EDTA, and incubated 10 min on ice. A working solution of 50\% Optiprep (iodixanol) (5 volumes 60\% Optiprep: 1 volumes $0.26 \mathrm{M}$ sucrose, $30 \mathrm{mM}$ MOPS, $1 \mathrm{mM}$ EDTA) and homogenization medium were mixed to prepare solutions for discontinuous gradients in Beckman SW55 tubes: $0.5 \mathrm{~mL}$ of $30 \%$ iodixanol on the bottom and $3.8 \mathrm{~mL}$ of $14.5 \%$ iodixanol, above which 1.2 ml EDTA-adjusted PMS was layered. Samples were spun at 45,000 rpm $\left(190,000 \times g_{a v}\right), 5 \mathrm{~h}$. A clear white band at the interface between the $30 \%$ iodixanol and the $14.5 \%$ iodixanol was collected as the DCV sample (fraction 9 of Western blot shown in Fig. 1A). The DCV sample was then extensively dialyzed in a cassette with $10,000 \mathrm{kD}$ molecular weight cutoff (24-48 h, $3 \times 5 \mathrm{~L})$ into the fusion assay buffer (120 mM potassium glutamate, $20 \mathrm{mM}$ potassium acetate, $20 \mathrm{mM}$ HEPES, $\mathrm{pH}$ 7.4). 


\subsection{Total internal reflection fluorescence microscopy}

Experiments were carried out on one of two microscopes. One was a Zeiss Axiovert 35 fluorescence microscope (Carl Zeiss, Thornwood, NY), equipped with a 63x water immersion objective $($ Zeiss; N.A. $=0.95)$ and a prism-based TIRF illumination. The light source was an OBIS 532 or 488 LS laser from Coherent Inc (Santa Clara Ca). Fluorescence was observed through a $610 \mathrm{~nm}$ band pass filter (D610/60; Chroma Battleboro, VT) by an electron multiplying CCD (DU-860E; Andor Technologies). The EMCCD was cooled to $-70^{\circ} \mathrm{C}$, and the gain was set between 200-220. The prism-quartz interface was lubricated with glycerol to allow easy translocation of the sample cell on the microscopy stage. The beam was totally internally reflected at an angel of $72^{\circ}$ from the surface normal, resulting in an evanescent wave that decays exponentially with a characteristic penetration depth of $\sim 100 \mathrm{~nm}$. An elliptical area of $250 \times 65$ $\mu \mathrm{m}$ was illuminated. The lase intensity, shutter, and camera were controlled by a homemade program written in LabVIEW (National Instruments, Austin, TX). The second set up was a Zeiss Axiovert 200 fluorescence microscope (Carl Zeiss, Thornwood, NY), with objective and TIRF set ups as described above. The light source was a $514 \mathrm{~nm}$ beam line of an argon ion laser (Innova 90C, Coherent, Palo Alto, CA), controlled through an acousto-optic modulator (Isomet, Springfield, VA), and a diode laser (Cube 640, Coherent) emitting light at $640 \mathrm{~nm}$. The characteristic penetration depth was $\sim 102 \mathrm{~nm}$ and $\sim 130 \mathrm{~nm}$ for the 514 and $640 \mathrm{~nm}$ lasers, respectively. An OptoSplit (Andor-Technologies, South Windsor, CT) was used to separate the fluorescence of different fluorophores. Fluorescence signals were recorded by an electron- 
multiplying charge-coupled device camera (iXon DV887ESC-BV, Andor, Belfast, UK). The EMCCD camera was cooled to $-70{ }^{\circ} \mathrm{C}$ and the electron gain factor was set between 200 and 240.

\subsection{Single proteoliposome fusion assay}

Acceptor plasma membrane SNARE complex containing planar supported bilayers were perfused with $3 \mathrm{~mL}$ of synaptobrevin-2 proteoliposomes $(\sim 0.6 \mu \mathrm{M}$ lipid) containing lipid and content labels. The fluorescence from the proteoliposomes was recorded with the 514 and 640 $\mathrm{nm}$ lasers using a EMCCD camera. After focusing the microscope in the first $60 \mathrm{~s}$ after injecting the synaptobrevin-2 proteoliposomes, images were recorded at a defined exposure time and were taken and spooled directly to the hard drive. Multiple spooling sets were taken for each bilayer and the averages are from the combination of all spooling data.

Single-vesicle fusion data were analyzed using a homemade program written in LabView (National Instruments). Stacks of images were filtered by a moving average filter. The intensity maximum for each pixel over the whole stack was projected on a single image. Vesicles were located in this image by a single-particle detection algorithm described in (Kiessling, et al., 2006). The peak (central pixel) and mean fluorescence intensities of a $5 \times 5$ pixel $^{2}$ area around each identified center of mass were plotted as a function of time for all particles in the image series. The exact time points of docking and fusion were determined from the central pixel (Domanska, et al., 2009). Cumulative distributions were determined from the time of docking to the time of fusion for individual fusion events and the fusion efficiency was determined from the 
number of vesicles that underwent fusion compared with the total number of vesicles that docked.

\subsection{Proteoliposome docking assay}

Different amount of proteoliposomes containing synaptobrevin-2 containing a lipid labeled fluorescent dye were injected into the planar supported bilayer chamber and the total amount of fluorescence in the TIRF field was monitored over time. Early images were analyzed to determine the average fluorescence signal per liposomes and this was used to convert fluorescence signal into the number of liposomes bound.

\subsection{Single DCV docking and fusion assay}

Acceptor t-SNARE protein containing planar supported bilayers were washed with fusion buffer

containing EDTA or divalent metal $\left(\mathrm{Ca}^{2+}\right.$ or $\left.\mathrm{Mg}^{2+}\right)$ as indicated in text. They were then perfused with DCV (50-100 $\mu \mathrm{L}$ depending on preparation) diluted into $2 \mathrm{~mL}$ of fusion buffer $(120 \mathrm{mM}$ potassium glutamate, $20 \mathrm{mM}$ potassium acetate, $20 \mathrm{mM}$ HEPES, $\mathrm{pH}$ 7.4) with additions to buffer as indicated in text. The fluorescence from DCVs was recorded the $532 \mathrm{~nm}$ laser using a EMCCD camera gain of 200-220. After injection of the DCV sample, the microscope was focused within no more than 30 seconds and then 5000 images were taken with 200 ms exposure times and spooled directly to the hard drive. One spooling set was taken for each bilayer. 
Single-vesicle fusion data were analyzed using a homemade program written in LabView (National Instruments). Stacks of images were filtered by a moving average filter. The maximum intensity for each pixel over the whole stack was projected on a single image. Vesicles were located in this image by a single-particle detection algorithm described in Kiessling et al. (Kiessling, et al., 2006). The peak (central pixel) and mean fluorescence intensities of a $5 \times 5$ pixel $^{2}$ area around each identified center of mass were plotted as a function of time for all particles in the image series. The exact time points of docking and fusion were determined from the central pixel similarly to pervious work (Domanska, et al., 2009). Cumulative distributions were determined from the time of docking to the time of fusion for individual fusion events and the fusion efficiency was determined from the number of vesicles that underwent fusion compared with the total number of vesicles that docked within 15 seconds of DCV docking.

DCV docking was normalized from each DCV preparation by running two bilayers in $100 \mu \mathrm{M}$ EDTA with syxtaxin-1a (183-288):SNAP-25 and using the average number of docked events in these experiments to normalize all other data collected for that preparation. Docking values from preparations of DCVs from wild-type, knockdown, and RNAi rescue cell lines cannot be compared to each other directly because normalizations were done within each preparation. However, trends of calcium acceleration are comparable. Experiments to inhibit proteins by antibody binding showed no major effect on docking in the absence of calcium, but an effect of synaptotagmin or CAPS knockdown on docking in the absence of calcium cannot be completely ruled out. 


\subsection{Single DCV Ca ${ }^{2+}$ triggered assay}

Acceptor t-SNARE containing planar supported bilayers were washed with fusion buffer containing $100 \mu \mathrm{M}$ EDTA and then incubated for 30 min with $0.5 \mu \mathrm{M}$ Munc18, $2 \mu \mathrm{M}$ complexin-1, and $100 \mu \mathrm{M}$ EDTA. DCV sample was then perfused in while keeping the concentrations of Munc18 and complexin-1 constant. After $30 \mathrm{~min}$, fusion buffer containing the indicated $\left[\mathrm{Ca}^{2+}\right]$ and $0.5 \mu \mathrm{M}$ soluble $\mathrm{Cy} 5$ dye was injected and fusion was monitored. The presence or absence of Munc18 and complexin-1 in the fusion buffer containing $\mathrm{Ca}^{2+}$ had no effect on the triggered fusion results.

\subsection{Western blotting}

Samples were separated by SDS-PAGE and transferred to nitrocellulose filters (Invitrogen, Grand Island, NY); membranes were washed with phosphate-buffered saline (PBS) and blocked with Odyssey Blocking Buffer (PBS). Primary antibodies were incubated in Odyssey Blocking Buffer with $0.1 \%(\mathrm{v} / \mathrm{v})$ Tween-20. The appropriate secondary antibody coupled with IRDye 800 was incubated in a dilution of 1 to 10,000 with the nitrocellulose filter. After extensive washing with PBS 0.1\% Tween-20 blots were imaged on an Odyssey Licore Imaging system (Lincoln, $\mathrm{NE}$ ).

\subsection{Ensemble lipid mixing assay}

Proteoliposomes containing SNAREs (syntaxin-1 and SNAP-25 or synaptobrevin-2) with FRET paired lipid probes $\left(1.5 \%\right.$ each of Rh-DOPE and NBD-DOPE) were incubated at $37{ }^{\circ} \mathrm{C}$ in fusion 
buffer with either EDTA or divalent metal $\left(\mathrm{Ca}^{2+}\right.$ or $\left.\mathrm{Mg}^{2+}\right)$ at indicated concentration and with any additional protein as indicated in text. After 10 minutes of incubation fluorimeter recording was started and DCV $(25-50 \mu \mathrm{L})$ or corresponding unlabeled SNARE proteoliposomes were added and NBD-dequenching was monitored. At the end of each experiment $0.1 \%$ Triton-X was added and the maximum NBD-dequenching was recorded and used to normalize each experiment.

\subsection{Cryoelectron microscopy}

Samples were applied to either c-flat or quantifoil holey carbon grids, blotted to near dryness, and plunged into a slurry of liquid ethane. Images were recorded at magnifications of $11,000 \mathrm{x}$ or $30,000 \mathrm{x}$ under low electron dose conditions $\left(\sim 20 \mathrm{e}^{-} / \AA^{2}\right)$ using a 4k x 4x CCD camera (Gatan, Pleasanton, CA) fitted to a Tecnai F20 electron microscope.

\subsection{Phosphate assay}

Lipid concentrations were assayed by the Bartlett phosphate method (Bartlett, 1959) modified as described in (Pokorny, et al., 2002).

\subsection{Protein assay}

The Pierce BCA protein assay kit from ThermoScientific was used according to manufacturer's directions. 


\subsection{EPR measurements}

\section{Protein spin-labeling and CW-EPR measurements}

These measurements were performed by Rafal Zdanowicz in the laboratory of David Cafiso on the protein complexin. All complexin-1 variants were labeled by addition of 10-fold molar excess of the thiol specific spin label, MTSL ((1-oxy-2,2,5,5-tetramethylpyrrolinyl-3-

methyl)methanethiosulfonate) and incubated overnight at $4^{\circ} \mathrm{C}$. Excess spin label was removed by desalting in physiological buffer (139 mM KCl, $12 \mathrm{mM} \mathrm{NaCl}, 20 \mathrm{MM} \mathrm{MOPS}, \mathrm{pH}=7.4)$ using the HiPrep 26/10 column (GE Healthcare Life Sciences). For sample preparation, protein or lipid aliquots were mixed in the specified molar ratios. Continuous-wave EPR measurements were performed on $6 \mu \mathrm{L}$ of sample loaded with Hamilton syringe into the $0.60 \mathrm{~mm} \times 0.84 \mathrm{~mm}$ (inner diameter $\mathrm{x}$ outer diameter) borosilicate glass capillaries (VitronCom). Spectra were recorded on a Bruker EMX X-band EPR spectrometer at $2 \mathrm{~mW}$ incident microwave swept through $100 \mathrm{G}$ and up to 30 scans were performed to increase the signal/noise ratio. Spectra were then processed using LabVIEW programs provided by Christian Alembic (University of California, Los Angeles, CA), normalized and plotted in OriginPro 7.5 (OriginLab Corporation).

\section{Binding affinities determined by EPR spectroscopy}

The binding affinity of spin-labeled complexin mutants to membranes or SNARE containing proteoliposomes was determined by EPR spectroscopy essentially as described previously (Victor \& Cafiso, 2001). In the present case, the normalized amplitudes of the EPR spectra, rather than the absolute amplitudes, were used to generate a plot of the fraction of membrane or SNARE bound complexin ( $\mathrm{fb}$ ) as a function of accessible lipid concentration. The data were fit 
to the expression: $\mathrm{fb}=\mathrm{K}[\mathrm{L}] /(1+\mathrm{K}[\mathrm{L}])$, where $[\mathrm{L}]$ is the accessible lipid concentration and $\mathrm{K}$ is the reciprocal molar partition coefficient.

\section{Power Saturation EPR}

Power Saturation EPR experiments were conducted at room temperature on a Bruker EMX Xband EPR spectrometer. $75 \mu \mathrm{M}$ complexin-1 was incubated with sonicated POPC:POPS small unilamellar vesicles and the sample was loaded into a gas=permeable TPX capillary. The microwave power was varied from 0.25 to $100 \mathrm{~mW}$ and 30 scans of the central peak were averaged for each power step. The applied magnetic field was swept through10 G with a modulation amplitude of $1 \mathrm{G}$ and frequency of $100 \mathrm{kHz}$. The power saturation was conducted on spin-labeled complexin-1 in the presence of air $\left(20 \% \mathrm{O}_{2}\right), \mathrm{N}_{2}$, or $\mathrm{N}_{2}$ with $10 \mathrm{mM} \mathrm{NiEDDA}$ (Victor \& Cafiso, 2001). In each of these conditions and for every power step, the amplitude of the central peak was measured and the $\mathrm{P}_{1 / 2}$ value was extracted using a LabVIEW program provided by Christian Altenbach (UCLA). The values of $\Delta \mathrm{P}_{1 / 2}\left(\mathrm{O}_{2}\right)$ or $\Delta \mathrm{P}_{1 / 2}(\mathrm{NiEDDA})$ were then determined from the difference in $\mathrm{P}_{1 / 2}$ values in the presence and absence of either $\mathrm{O}_{2}$ or NiEDDA, respectively. A depth parameter, $\Phi$, related to the local concentrations of $\mathrm{O}_{2}$ and NiEDDA, which vary as a function of depth in the lipid bilayer (Altenbach, et al., 1994), was determined from the values of $\Delta \mathrm{P}_{1 / 2}$. Based on a defined calibration curve, the obtained values of depth parameter were applied in the hyperbolic tangent function that describes behavior of $\Phi$ relative to distance from the membrane surface (Frazier, et al., 2002). 


\subsection{Fluorescence anisotropy measurements}

Native cysteine C105 of complexin-1 was fluorophore-labeled with Alexa546. Experiments were performed on a FluoroMax-3 spectrofluorometer (Horiba Scientific). Fluorescence anisotropy of complexin was measured in a $1.2 \mathrm{~mL}$ volume containing $50 \mathrm{nM}$ complexin before and after addition of proteoliposomes with reconstituted proteins as indicated in text at lipid: protein of 400. Protein-free vesicles were used as a control sample, where the same lipid concentration as the proteoliposomes was added.

\subsection{NMR spectroscopy}

All NMR experiments presented in the manuscript were performed by Binyong Liang. TROSYversions of 3D backbone experiments (Salzmann, et al., 1998) [HNCA, HN(CA)CB, HNCO, $\mathrm{HN}(\mathrm{CA}) \mathrm{CO}]$ were performed on ${ }^{2} \mathrm{H},{ }^{13} \mathrm{C},{ }^{15} \mathrm{~N}$-labeled full-length complexin in buffer $(10 \mathrm{mM}$ each HEPES, MES < and Acetate $\mathrm{pH} 6,150 \mathrm{mM} \mathrm{NaCl}, 1 \mathrm{mM}$ EDTA) or in DPC (the same buffer with the addition of $150 \mathrm{mM}$ DPC), and ${ }^{13} \mathrm{C},{ }^{15} \mathrm{~N}$-labeled complexin (26-83) in buffer (10 mM each HEPES, MES and Acetate $\mathrm{pH}$ 6, $150 \mathrm{mM} \mathrm{NaCl}, 1 \mathrm{mM}$ EDTA). Data were collected on

a Bruker Advance $800 \mathrm{MHz}$ spectrometer at $25^{\circ} \mathrm{C} .{ }^{15} \mathrm{~N}$ labeled complexin samples were employed in the study with Nano disc and vesicle interactions. ${ }^{15} \mathrm{~N}$-labeled complexin (26-83) samples were employed in interaction studies with the soluble binary complex. Protein-free nanodiscs were assembled with POPC and three different membrane scaffold proteins (MSP1D1, MSP1D1- $\Delta \mathrm{H} 5$, and MSP1D1- $\Delta \mathrm{H} 4 \Delta \mathrm{H} 5)$, and subsequently purified according to published protocols (Hagn, et al., 2013). Protein-free nanodiscs were added to complexin samples in NMR buffer to make final NMR samples with protein:MSP:lipid ratios of 1:2:120. All three different 
MSP samples resulted in very similar NMR spectra, and the data from MSP1D1 nanodisc sample was used in data shown here. Small POPC:POPG (90:10) vesicles were formed by freeze/thaw//sonicate cycles, and added to a complexin NMR sample in buffer to make a final sample with a protein:lipid ratio of 1:50. These 2D spectra were collected on either the $800 \mathrm{MHz}$ or a $600 \mathrm{MHz}$ Bruker Advance spectrometer. All spectra were processed and analyzed with NM Pipe (Delaglio, et al., 1995) and Sparky (Goddard \& Kneller, n.d.). 


\section{Chapter 3: Single Vesicle Fusion with a Planar}

\section{Supported Bilayer}

Kreutzberger, A.J.B., V. Kiessling, and L.K. Tamm (2015) Biophysical Journal, 109:319-329.

Kreutzberger, A.J.B., V. Kiessling, B. Liang, P. Seelheim, S. Jakhanwal, R. Jahn, J.D. Castle, and L.K. Tamm (submitted).

Mathematical models for diffusion of sulforhodamine B and for NPY-Ruby were derived with the assistance of Volker Kiessling and are published in (Kreutzberger, et al., 2015) and (Kreutzberger, et al., Submitted) respectively.

\subsection{Summary}

Membrane fusion has been reconstituted using both ensemble and single particle fusion assays. Single vesicle methods allow docking and fusion to be separate while distinguishing between hemi- and full-fusion. An asymmetric planar supported bilayer containing t-SNAREs can be formed using the Langmuir-Blodgett/vesicle fusion method. This results in oriented SNARE proteins that are laterally mobile which can fuse with syb2 proteoliposomes in a SNARE specific manner. The incorporation of a content fluorescent dye shows that full fusion events result in a fusion pore with a characteristic 2-dimensional (2D) diffusion of the dye under the cleft of the supported membrane. The membrane dye allows the observation of both the full fusion events as 
well as hemifusion events by indicating if the outer leaflet or both leaflets of the syb2 proteoliposome merge with the planar supported bilayer. Purified dense core vesicles from cell culture were also shown to be capable of fusion with the planar supported bilayer which could be monitored using neuro-peptide Y tagged to a fluorescent protein (NPY-Ruby). In this chapter methods of planar supported bilayer formation, syb2 proteoliposome fusion, and DCV fusion will be described and characterized.

\subsection{Introduction}

Planar supported bilayers have been used to as a model system to study membrane fusion since 1994 when it was used to reconstitute fusion mediated by the hemagglutinin protein from influenza (Hinterdorfer, et al., 1994). Since then, planar bilayers have been used to study the mechanism of viral and SNARE mediated membrane fusion. To incorporate SNARE proteins into planar supported bilayers two distinct methods of bilayer formation have been employed. The first method allows proteoliposomes to directly rupture on a surface to form the planar bilayer (Fix, et al., 2004). The second is a two-step method with the first step being for formation of a lipid monolayer using a Langmuir-Blodgett trough followed by incubation of proteoliposomes that spontaneously form the outer leaflet of the planar bilayer (Domanska, et al., 2009; Hinterdorfer, et al., 1994; Wagner \& Tamm, 2001), which will be referred to as the monolayer fusion method. The monolayer fusion method is currently the only method in the SNARE mediated planar supported bilayer fusion field that results in an asymmetric membrane (Crane, et al., 2005) with oriented proteins (Liang, et al., 2013; Hinterdorfer, et al., 1994), that are laterally mobile (Wagner \& Tamm, 2001; Domanska, et al., 2009; Hinterdorfer, et al., 1994), 
and results in fusion event where the content is transferred through pore in the planar bilayer and then diffuses laterally in the cleft under the planar bilayer (Kreutzberger, et al., 2015).

\subsection{Formation of Planar Supported Bilayers}

Several methods for forming a planar supported bilayer on a hydrophilic surface have been developed. These include consecutively deposited two monolayers onto a hydrophilic substrate (Tamm \& McConnell, 1985), the direct fusion method when lipid vesicles are ruptured on a hydrophilic substrate (Brian \& McConnell, 1984), and the monolayer-fusion technique where a Langmuir-Blodgett monolayer is deposited to form the inner leaflet of the bilayer and then lipid vesicles are incubated on this surface and spontaneously rupture to form the outer leaflet of the planar bilayer (Kalb, et al., 1992). The monolayer fusion method has the benefit in allowing the composition of the inner and outer leaflet to be controlled and appears to be able to orient proteins with larger domains away from the substrate (Hinterdorfer, et al., 1994; Liang, et al., 2013). Both the vesicle fusion method and the monolayer fusion method have been used to study SNARE mediated membrane fusion. 


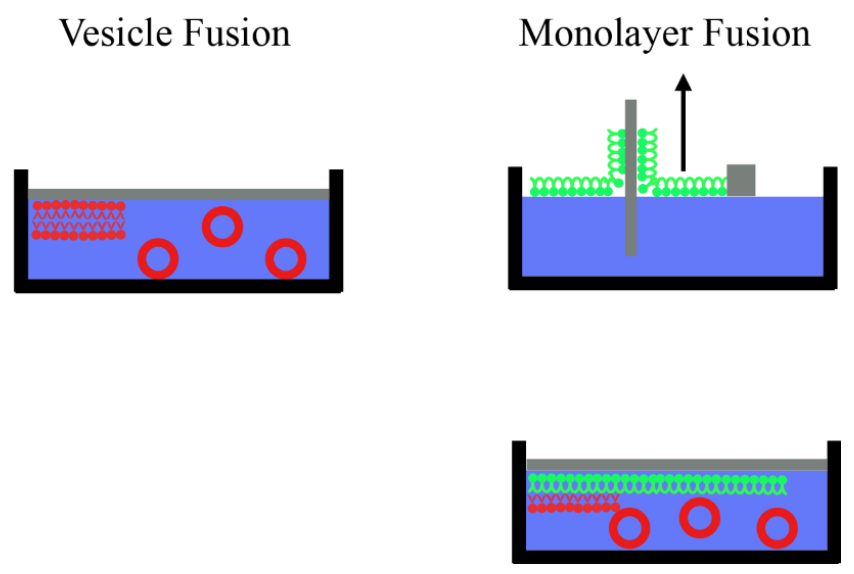

Figure 3.1: Methods for preparing planar supported bilayers for SNARE mediated fusion assays. The vesicle fusion method is when lipid vesicles are ruptured directly onto a surface. The monolayer fusion method of depositing a monolayer onto a surface then forming the outer leaflet of the bilayer by the spontaneous rupture of lipid vesicles.

\subsection{SNARE-Mediated Single Vesicle Fusion with Planar Supported Bilayer}

SNARE proteins have been incorporated into planar supported bilayers using both the direct fusion method (Fix, et al., 2004) and the monolayer-fusion technique (Wagner \& Tamm, 2001), Figure 3.1. The direct fusion method was shown to have several problems which include that SNAP-25 was not needed for docking or fusion, fusion was induced by the heating of the sample by the laser, and fusion required divalent ions (Bowen, et al., 2004; Fix, et al., 2004). Some of these problems were overcome; but studies using content dye revealed that a SNARE dependent rupturing event was occurring instead of the expect fusion event (Wang, et al., 2009). The direct fusion method was shown to lack mobile proteins (Bowen, et al., 2004) which is likely an indicator of planar supported bilayer quality. 
Reconstitution of SNARE proteins using the monolayer-fusion method results in mobile proteins (Domanska, et al., 2009; Wagner \& Tamm, 2001) that had SNARE dependent docking and fusion (Domanska, et al., 2009; Kreutzberger, et al., 2016). Proteoliposomes containing syb2 dock to planar supported bilayers containing t-SNAREs reconstituted using the monolayer-fusion method, Figure 3.2 (A and B). Single syb2 proteoliposomes can undergo four characteristic types of events being docking, fusion, hemifusion, and a two-step hemi- to full- fusion, Figure 3.3. 
a)

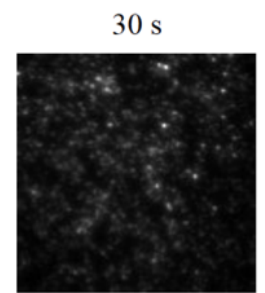

$990 \mathrm{~s}$
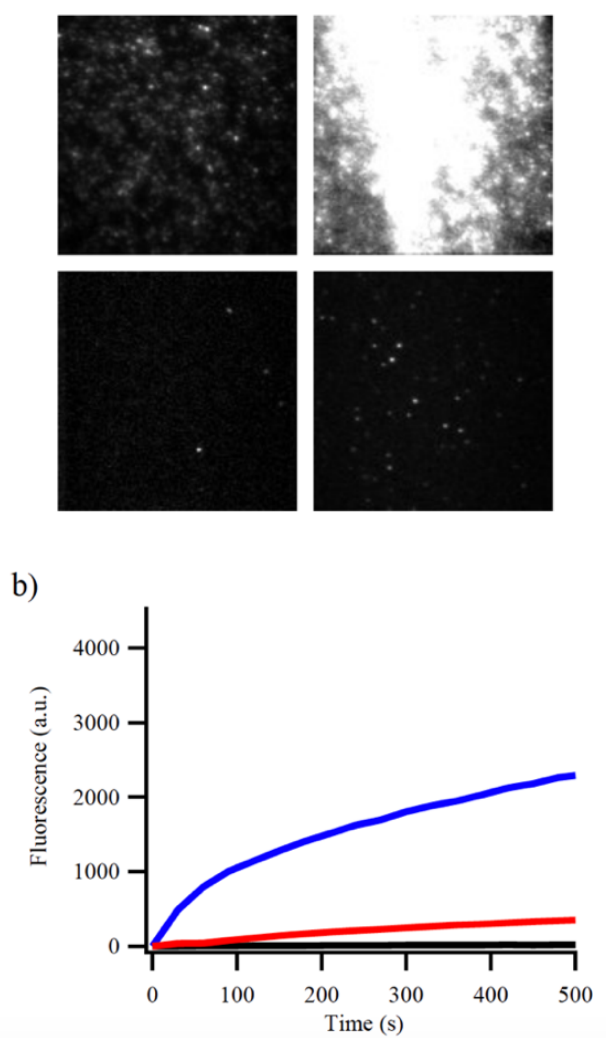

Figure 3.2: Docking of syb2 proteoliposomes ( $5 \mu \mathrm{M}$ lipid) to a planar supported bilayer. Conditions shown are for planar bilayer (with bPC lipids) with $\triangle \mathrm{N}$ complex t-SNAREs (lipid:protein of 1000) and proteoliposomes containing syb2 (lipid:protein 400, with a lipid composition of 54:20:5:20:1 bPC:bPE:bPS:chol:Rh-DOPE). (A) Representative images of docking 30 seconds after assay has begun (left column) and well after saturation has occurred (right column). High docking is observed (top row) in normal conditions and no docking is observed when the syb96 inhibitor peptide $(2 \mu \mathrm{M})$ was added as a control (bottom row). (B) Fluorescence in the TIRF field over time indicating syb2 proteoliposomes interacting with the planar supported bilayer. High syb2 proteoliposome docking is observed when both t-SNARE and v-SNAREs are present (blue), but very little docking is observed in the absence of t-SNAREs (red) or in the presence of the syb96 peptide (black). Figure is adapted from (Kreutzberger, et al., 2015). 
I.

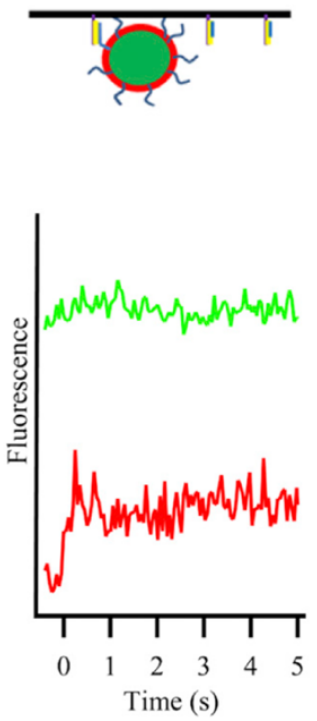

II.
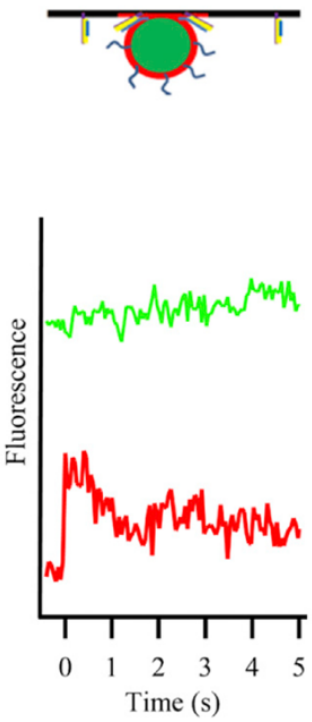

III.
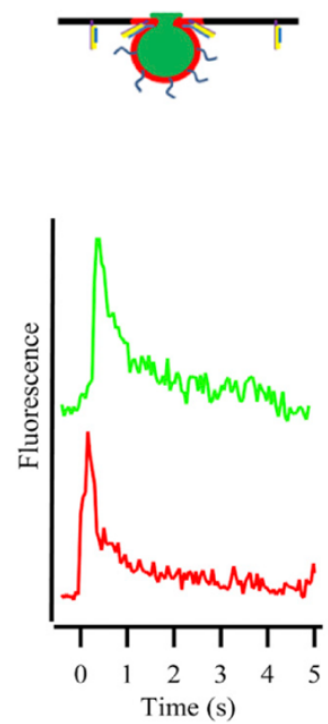

IV.
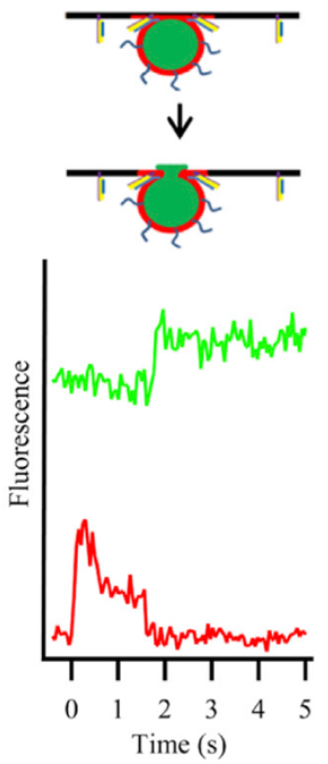

Figure 3.3: Four characteristic types of events observed in a single-vesicle fusion assay. The first row is a representative illustration of each type of event. The green (upper) traces are fluorescence intensity traces of the sulforhodamine B content dye and the red (lower) traces are fluorescence intensity traces of the DiD membrane dye. (I) Docking of syb2 proteoliposomes is characterized by an increase in the membrane dye fluorescence caused by the liposome entering the TIRF field. This trace remains constant with no changes after the initial docking. Only a low background signal is observed for the content dye because sulforhodamine $B$ is included at self-quenching concentrations in these experiments. (II) hemifusion of syb2 proteoliposomes is characterized by a decrease of the membrane dye fluorescence to approximately half of the docked fluorescence signal. The content dye does not dequench in these events. (III) Direct full fusion of syb2 proteoliposomes is characterized by a decrease of the membrane dye fluorescence to baseline levels. The decrease of the membrane dye signal is paralleled by an increase of the content dye fluorescence that is attributable to dequenching, followed by a decrease attributable to diffusion of the dye into the cleft between the supported membrane and support. (IV) Occasionally, after a hemifusion event has been observed and the membrane dye fluorescence has leveled at approximately half-full intensity, a second decrease of the membrane dye fluorescence back to the background level occurs. This second drop of the signal is paralleled by a dequenching of the content dye. The four shown events are not plotted to scale attributable to differences in individual liposomes sizes, their positions in the no uniformly illuminated TIRF field and other optical and dye variations. This figure is adapted from (Kreutzberger, et al., 2015). 
Upon fusion of syb2 proteoliposomes with the planar bilayer an encapsulated content dye dequenches and then the fluorescence decays, Figure 3.3. The decay of the content dye follows the excepted decay of a 2D diffusion model, Figure 3.4. Content transfer from a vesicle into the cleft between a planar supported bilayer and its substrate can be modeled by two-dimensional diffusion from a point source by calculating the absolute integrated fluorescence intensity within a cylindrical volume of radium $R$ centered on the release point and height $h$ in a cylindrical coordinates:

$$
F(t)=A \int_{0}^{R} 2 \pi \rho d \rho \int_{0}^{h} C(\rho, z, t) \phi_{\text {rel }}(\rho, z, t) I_{\text {laser }}(z) d z
$$

The radial coordinate centered on the $\mathrm{z}$ axis is $\rho$ and $\mathrm{z}=0$ is defined as the substrate surface, $\mathrm{C}$ is the concentration of the dye as a function of position and time, and A is an unknown proportionality constant. $I_{\text {laser }}(\mathrm{z})$ is the intensity profile from TIRF excitation which is $I_{\text {laser }}(\mathrm{z})=$ $I_{0} \exp \left(-z / z_{0}\right)$, where $\mathrm{z}_{0}$ is the characteristic decay length of the evanescent field, i.e., $102 \mathrm{~nm}$ in our case. $\phi_{r e l}$ is the relative concentration dependent quantum yield of the fluorophore, which is determined by measuring the fluorescence intensity per molecule across a range of dye concentratiopns on the TIRF microscope (Wang, et al., 2009; Rawle, et al., 2011). The fit of the exponential decay of sulforhodamine B on our set up was $\phi_{r e l}=e^{-74 C(\rho, z, t)}$. The model for two-dimensional diffusion of the dye in the cleft is

$$
C(\rho, t)=\frac{C_{0} V_{v e s}}{4 \pi h D t} e^{-\rho^{2} / 4 D t}
$$

When a vesicle burst, the dye diffuses in 3D into a half-space above the vesicle and the corresponding model for three-dimensional diffusion

$$
C(\rho, z, t)=\frac{2 C_{0} V_{v e s}}{(4 \pi D t)^{3 / 2}} e^{-\left(\rho^{2}+z^{2}\right) / 4 D t}
$$


needs to be inserted into eq. 3.1. Our initial concentrations $\mathrm{C}_{0}$ of sulforhodamine $\mathrm{B}$ in the vesicle were $100 \mathrm{mM}$ in both cases assuming that $100 \%$ of the dye is encapsulated in vesicles of volume $\mathrm{V}_{\mathrm{ves}}=3.88 \times 10^{-10} \mathrm{~m}^{2} / \mathrm{s}$ (Gendron, et al., n.d.).

As done previously (Rawle, et al., 2011), the fluorescence intensities were normalized and the decay profiles were used to distinguish between two- and three-dimensional diffusion. Mathematica was used to perform the numerical integrations of the normalized fluorescence intensity for each diffusion model. The integration in $\rho$ went from 0 to $R=625 \mathrm{~nm}$. The integration in $\mathrm{h}$ went from 0 to $2 \mathrm{~nm}$ for $2 \mathrm{D}$ diffusion in the cleft and from $6 \mathrm{~nm}$ to $2 \mu \mathrm{m}$ for $3 \mathrm{D}$ diffusion into the half-space above the vesicle, respectively. The solid lines are simulations using these models with $100 \mathrm{mM}$ and $50 \mathrm{mM}$ sulforhodamine $\mathrm{B}$ as indicated. The reason that the fit using $\mathrm{C}_{0}=50 \mathrm{mM}$ is better may be rationalized by the fact that the dye is not fully encapsulated during preparation on the gel filtration column. 


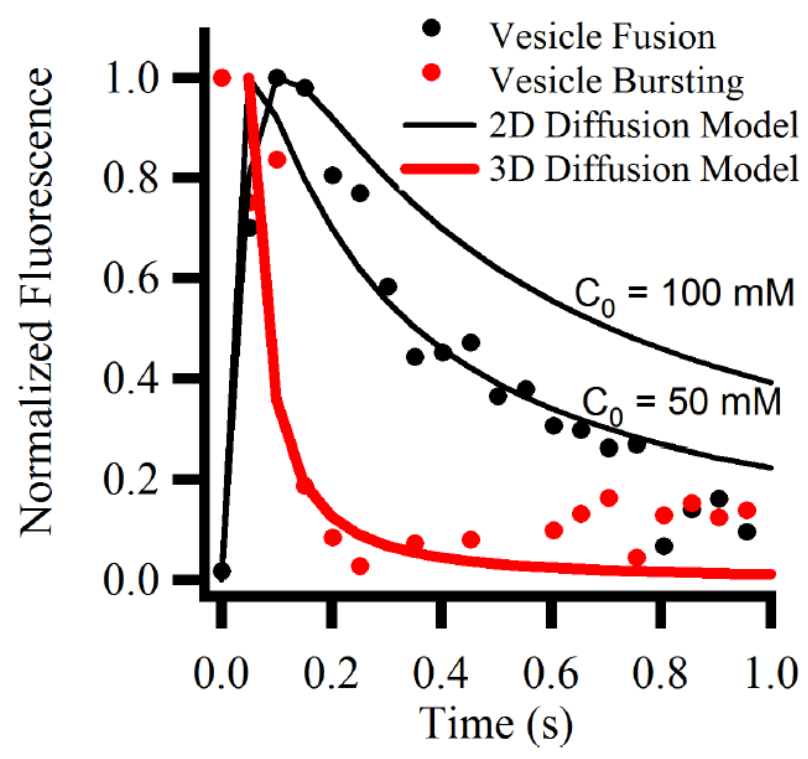

Figure 3.4: Decay of sulforhodamine B fluorescence from a single vesicle due to diffusion into the cleft between the supported membrane and the quartz substrate after controlled fusion of the vesicle with the supported membrane (black data points) and from a single vesicle into the surrounding buffer after bursting of the vesicle on a lipid monolayer of bPC:chol (80:20) (red data points). The solid lines are simulations of a 2D dye diffusion model (black lines) and a 3D dye diffusion model (red line) according to the theory of (Wang, et al., 2009) with a typical diffusion coefficients as described below. This figure is adapted from (Kreutzberger, et al., 2015).

While the content dye indicates full fusion the membrane dye allows for hemifusion to be distinguished, Figure 3.3. The decrease in the membrane dye (DiD) was measured and revealed that the dye decreased by about $60 \%$ for hemifusion indicating the outer leaflet of the syb 2 proteoliposome merged with the planar bilayer, Figure 3.5. The total amount of dye diffusion was measured for several conditions of different concentrations of cholesterol and the correlation between measurements utilizing the membrane dye and the content dye showed that the membrane dye is sufficient to distinguish between both hemi- and full-fusion, Figure 3.5. 

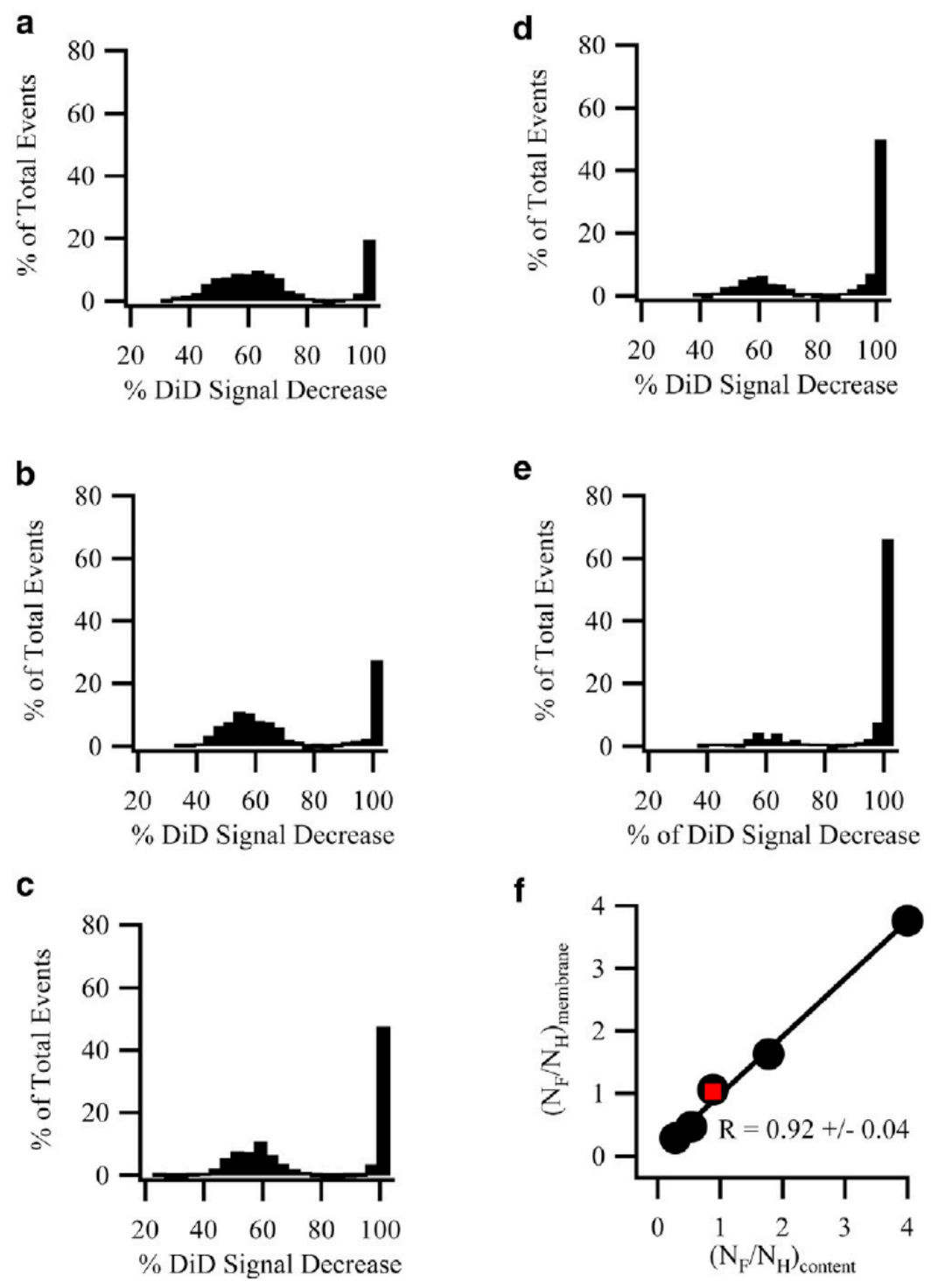

Figure 3.5: Histograms of DiD label diffusing into the planar target membrane after single syb2 proteoliposome fusion events with (A) 0 , (B) 10, (C) 20, (D) 30, and (E) $40 \mathrm{~mol} \%$ cholesterol in the planar target membranes. Fluorescence intensities of each fusion event were measured before syb2 proteoliposomes docking, after docking, and after fusion. (F) Correlation of the percent membrane dye diffusion into the planar membrane. The correlation coefficient is $0.92 \pm 0.04$. The red square represents an independent experiment using $20 \%$ cholesterol where only a rhodamine-DOPE membrane label was used instead of the DiD membrane and sulforhodamine B content labels, indicating that the DiD signal is not contaminated by bleed-through from sulforhodamine $B$ fluorescence. This figure is adapted from (Kreutzberger, et al., 2015). 


\subsection{Single vesicle fusion with physiological vesicles}

Fusion of physiological vesicles has been shown for purified synaptic vesicles (SV) from rat brains (Holt, et al., 2008; Park, et al., 2012; Kiessling, et al., 2013) and purified dense core vesicles (DCVs) from bovine chromaffin cells (Park, et al., 2012; Park, et al., 2015). SV and DCVs exhibit accelerated lipid mixing in the presence of calcium (Holt, et al., 2008; Park, et al., 2012) and this was shown to be caused by an increase and acceleration of fusion when SV were observed in the single vesicle planar supported bilayer fusion assay (Kiessling, et al., 2013). These procedures are limited in that they cannot include a content label in the physiological vesicle and the protein level of these vesicles cannot be manipulated easily because of being prepared from animal sources.

Preparing physiological vesicles from an immortalized cell line allows these advantages to be overcome. An iso-osmotic fractionation of DCVs was developed from PC12 cells, described in materials and methods section 2.7 , which results in a DCV sample with minimal contamination, Figure 3.6, that is active in an ensemble lipid mixing assay containing reconstituted t-SNAREs, Figure 3.7. Transfection of DCV with neuro-peptide Y tagged with the fluorescent protein mRuby (NPY-mRuby) allows fusion of DCVs to be observed with the planar supported bilayer, Figure 3.8. 


\section{Fraction Number \\ $\begin{array}{lllllllllll}\text { PNS PMS } & 1 & 2 & 3 & 4 & 5 & 6 & 7 & 8 & 9 & 10\end{array}$

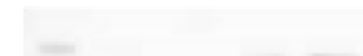

SQR

Secretogranin

Calnexin

Synaptotagmin-1

Synaptotagmin-9

CAPS-1

CAPS-2
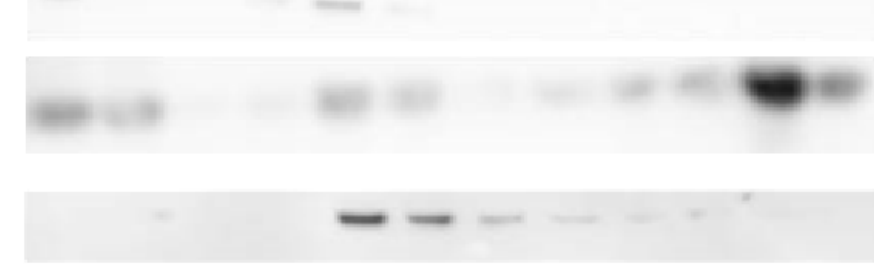

Figure 3.6: Western blots of post nuclear supernatant (PNS), post mitochondrial supernatant (PMS) and individual fractions of the iodixanol gradient. Fraction 9 containing the DCV content marker secretogranin was collected as DCVs. DCVs are also enriched in the calcium interacting proteins synaptotagmin-1, synaptotagmin-9, CAPS-1, and CAPS-2. Contamination by mitochondria (SQR, succinate-ubiquinone oxidoreductase) and endoplasmic reticulum (calnexin) is minimally detected in the DCV fraction. This figure is adapted from (Kreutzberger, et al., Submitted). 


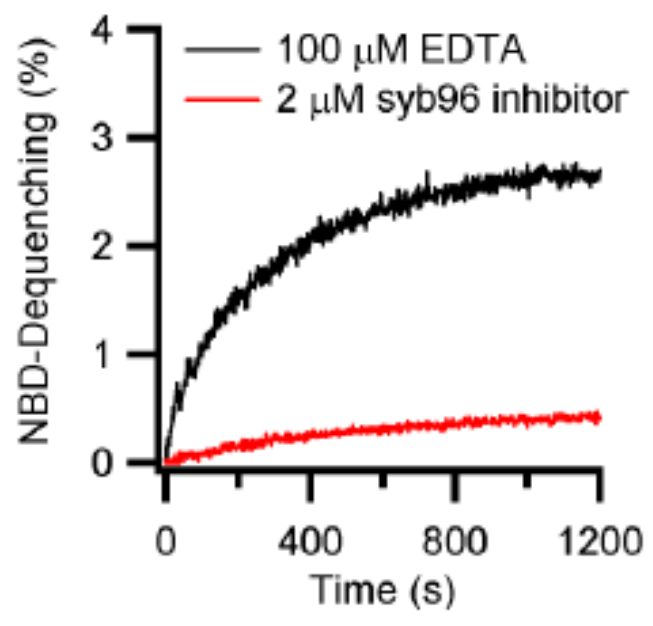

Figure 3.7: Fusion of purified DCVs with liposomes containing syntaxin-1a (residues 183-288): SNAP-25 at a lipid:protein ratio of 500 (black) and a lipid composition of bPC:bPE:bPS:Chol: PI:PI4,5P ${ }_{2}:$ Rh-DOPE:NBD-DOPE (23.5:23.5:15:30:4:1:1.5:1.5). Lipid mixing (NBD dequenching) traces shown are averages of 4 repeated experiments. The presence of synaptobrevin-2 (residues 196) inhibitor peptide ( $2 \mu \mathrm{M})$ abolishes lipid mixing (red). This figure is adapted from (Kreutzberger, et al., Submitted).

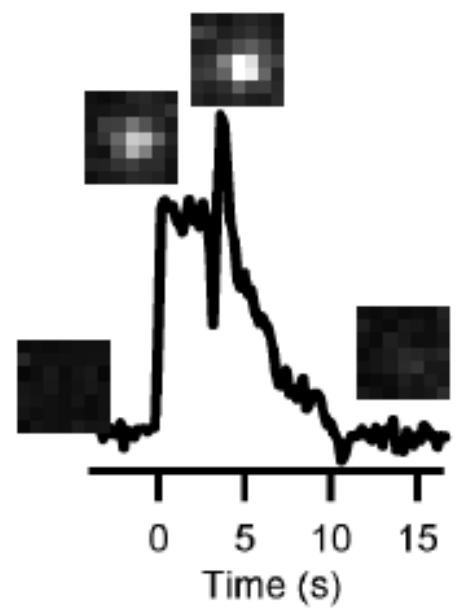

Figure 3.8: Single fusion event of NPY-Ruby labeled DCV with a planar supported bilayer containing syntaxin-1a (residues 183-288):SNAP-25 (lipid:protein ratio of 3000). This figure is adapted from (Kreutzberger, et al., Submitted). 
The fluorescence signal originating from DCVs during fusion with the planar bilayer follows a characteristic line shape, Figure 3.8 and 3.9. In the following paragraphs, we describe a simple 2-step fusion/diffusion model that reproduces the basic features of the signal.

At each time the fluorescence originating from the fluorophore mRuby is determined by the sum of the fluorophore fraction located in the lumen of the DCV at a concentration $\mathrm{C}_{\mathrm{DCV}}$ and the fraction in the small cleft between supported membrane and substrate at concentration $\mathrm{C}_{\mathrm{CLEFT}}$ :

$$
I=I_{D C V}\left(C_{D C V}(t, x, y), \lambda\right)+I_{C L E F T}\left(C_{C L E F T}(t, x, y, D) \lambda\right)
$$

The model starts with a DCV of diameter $d_{D C V}=200 \mathrm{~nm}$ (Zhang, et al., 2011) docked at the supported lipid bilayer at distance $\mathrm{z}_{0}=8 \mathrm{~nm}$ (Kiessling \& Tamm, 2003) from the substrate and at $\mathrm{x}, \mathrm{y}=0$. For the observed intensities, we take into account the $2 \mathrm{D}$ point spread function at $\lambda=$ $600 \mathrm{~nm}$ and the decay of the evanescent wave with a characteristic penetration depth of $\mathrm{d}_{\mathrm{p}}=100$ nm:

$$
I_{0}=I_{D C V}\left(t<t_{1}\right)=P S F(\lambda) \cdot \int_{z_{0}}^{z_{0}+d_{D C V}} C_{D C V}(x, y, z) e^{\frac{-z}{d_{p}}} d z
$$

At time $t_{1}$ a fusion pore opens and content from the DCV gets released through the supported membrane into the cleft with a characteristic rate $\mathrm{k}_{\mathrm{r}}$ at $\mathrm{x}, \mathrm{y}=0$ :

$$
r\left(t-t_{1}\right) e^{-\frac{\left(t-t_{1}\right)}{k_{r}}}
$$


Fluorescent content in the cleft is located at an average distance $\mathrm{z}_{\mathrm{CLEFT}}=2 \mathrm{~nm}$ (Kiessling \& Tamm, 2003) and spreads laterally in the $\mathrm{x}, \mathrm{y}$ plane by free diffusion characterized by a diffusion coefficient $\mathrm{D}_{1}$.

$$
\begin{aligned}
& d C_{C L E F T}\left(t_{1}<t<t_{2}\right)=\left[d r+D_{1} \Delta C_{C L E F T}\right] d t \\
& I_{C L E F T}\left(t_{1}<t<t_{2}\right)=P S F(\lambda) \cdot C_{C L E F T}(x, y, z, t) e^{\frac{-z_{C L E F T}}{d p}}
\end{aligned}
$$

During the life time of the fusion pore $\left(t_{1}<t<t_{2}\right)$ the shape of the DCV stays intact and content gets released from membrane proximal areas first (Figure 3.9). A simpler model in which the distribution of content inside the DCV stays homogenous did not fit the data sufficiently. The fluorescence intensity originating from the DCV during this phase becomes:

$$
I_{D C V}\left(t_{1}<t<t_{2}\right)=P S F(\lambda) \cdot \int_{z_{1}}^{z_{0}+d_{D C V}} C_{D C V}(x, y, z) e^{\frac{-z}{d_{p}}} d z
$$

with $z_{1}(t)$ changing over time as more and more content gets released.

At time $t_{2}$ the DCV with its remaining content in the distal region from the supported membrane collapses into the supported lipid bilayer and diffuses together with the already released content laterally within the cleft with a diffusion coefficient $\mathrm{D}_{2}$.

$$
\begin{aligned}
& C_{C L E F T}\left(t=t_{2}\right)=C_{C L E F T}\left(x, y, z, t_{2}\right)+C_{D C V}\left(x, y, z, t_{2}\right) \\
& d C_{C L E F T}\left(t>t_{2}\right)=D_{2} \Delta C_{C L E F T} d t
\end{aligned}
$$


At the time of collapse $\left(\mathrm{t}_{2}\right)$ we assume the remaining content to collapse into a plane corresponding to the surface area of the original DCV instantaneously. The total observable intensity which now originates only from the cleft becomes:

$$
I_{C L E F T}\left(t>t_{2}\right)=\operatorname{PSF}(\lambda) \cdot C_{C L E F T}(x, y, z, t) e^{\frac{-z C L E F T}{d p}}
$$

We simulated the fluorescence intensity of the central pixel centered at a DCV using the above parameters and adjusting the length of the time period $t_{2}-t_{1}$, the release rate $k_{r}$ and the diffusion coefficients $\mathrm{D}_{1 / 2}$. DCVs, releasing fluorescent content at a rate of $0.7 \mathrm{~s}^{-1}$ for $700 \mathrm{~ms}$ that diffuses with a rate of $D_{1}=5 \mu \mathrm{m}^{2} / \mathrm{s}$ and then collapses into the supported membrane after which the remaining content diffuses with a specific diffusion coefficient of $\mathrm{D}_{2}=0.05 \mu \mathrm{m}^{2} / \mathrm{s}$ were used to compute traces for TIRF microscopy $\left(d_{p}=100 \mathrm{~nm}\right)$ in Figure 3.9A and epi-fluorescence microscopy $\left(d_{p}=\right.$ in) in Figure 3.9B. Despite the simplifications in the model the curves reproduce the basic characteristics of the recorded traces very well. Both diffusion coefficients are significantly smaller than the reported diffusion coefficient for GFP in solution $(\mathrm{D} \approx 80$ $\mu \mathrm{m}^{2} / \mathrm{s}$, (Zareh, et al., 2012)) indicating that the content indeed diffuses in the cleft between supported lipid bilayer and substrate where the molecular mobility is known to be impaired (Fromherz, et al., 1999; Kiessling, et al., 2000). The slower diffusion observed after the collapse of the DCV might be due to the high density of (protein-) material at the fusion site. The epifluorescence recording in Figure 3.9B shows that the characteristic peak observed in the TIRF recording, Figure 3.9A, is indeed caused by the movement of fluorescent content closer to the surface within the evanescent field. As mentioned above, it was necessary to preserve the overall structure and shape of the DCV during the release phase to model the data sufficiently. 
This observation agrees well with the explanation for the amperometric foot signals during exocytotic events in chromaffin cells (Albillos, et al., 1997).
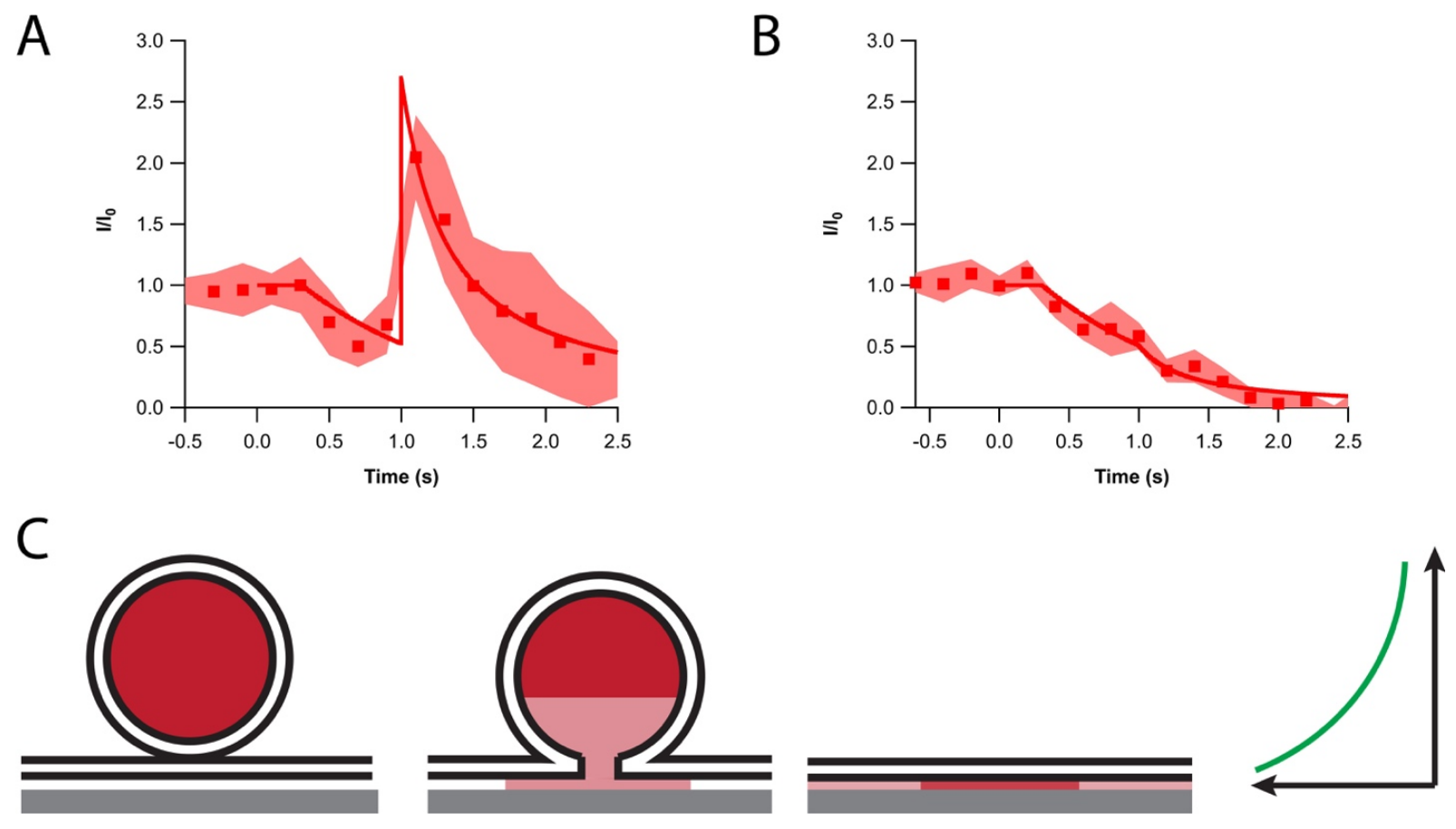

Figure 3.9: Line shape of intensity traces from single DCV fusion events. (A) Average peak pixel intensities (squares, with standard deviations shown as shades) from 10 individual DCV fusion events. The signals were aligned at the characteristic peak at $t=1 \mathrm{~s}$. DCV fusion was observed by TIRF microscopy as described in the Methods section. (B) Averaged peak pixel intensities (squares, with standard deviations shown as shades) from 5 individual DCV fusion events observed on an EPI fluorescence microscope. In this case, DCVs were docked under conditions of syntaxin1a (1-288):SNAP-25 (lipid:protein of 3000) incubated with $0.5 \mu \mathrm{M}$ Munc18 and $2 \mu \mathrm{M}$ complexin-1. The undocked DCVs were washed out and fusion was triggered with $\mathrm{Ca}^{2+}$ and monitored using an EPI fluorescence microscope. (C) 2-step fusion/diffusion model to simulate the single DCV fluorescence data which is described in detail in the Supplemental Text. The results of the simulations are shown in (A) and (B) as solid lines. This figure is adapted from (Kreutzberger, et al., Submitted). 


\subsection{Discussion}

The characteristics of protein mobility, protein orientation, requirements of cognate SNARE partners, the transfer of a membrane dye into the planar supported bilayer from the syb2 proteoliposomes, and the release of a content dye from the syb2 proteoliposome under the plane of the supported bilayer indicate that planar supported bilayers made by the monolayer fusion method are an excellent model system to study SNARE mediated exocytosis. This assay has successfully been adapted for fusion with synaptic vesicles (Kiessling, et al., 2013) and here we present fusion of DCVs with fluorescently tagged proteins labeling the secretory vesicle interior. This planar supported bilayer is used with reconstituted syb2 proteoliposomes and DCVs to characterize t-SNARE purification procedures (Chapter 4), membrane curvature on fusion (Chapter 5), cholesterol role in membrane fusion (Chapter 6), the mechanism of complexin-1 inhibition of spontaneous fusion (Chapter 7), and the role of accessory proteins in regulated calcium triggered fusion (Chapter 8). 


\section{Chapter 4: Assembly and comparison of plasma membrane SNARE acceptor complexes}

Kreutzberger, A.J.B., B. Liang, V. Kiessling, and L.K. Tamm, (2016) Biophysical Journal $110: 2147-2150$

Monomeric syntaxin-1a purification was developed by Binyong Liang, who purified and assembled all proteins used in this study. All fusion experiments were performed by Alex Kreutzberger.

\subsection{Summary}

Neuronal exocytotic membrane fusion occurs on a fast timescale and is dependent on interactions between the vesicle SNARE synaptobrevin-2 and the plasma membrane SNAREs syntaxin-1a and SNAP-25 with a 1:1:1 stoichiometry. Reproducing fast fusion rates as observed in cells by reconstitution in vitro has been hindered by the spontaneous assembly of a $2: 1$ syntaxin-1a:SNAP-25 complex on target membranes that kinetically alters the binding of synaptobrevin-2. Previously, an artificial SNARE acceptor complex consisting of 1:1:1 syntaxin-1a (residues 183-288):SNAP-25:syb(residues 49-96) was found to greatly accelerate the rates of lipid mixing of reconstituted target and vesicle SNARE proteoliposomes. Here two new procedures to assemble membrane-bound 1:1 SNARE acceptor complexes that produce fast and efficient fusion without the need of the synaptobrevin-2 (49-96) peptide. In the first procedure, syntaxin-1a is purified in a strictly monomeric form and subsequently assembled with SNAP-25 
in detergent with the correct 1:1 stoichiometry. In the second procedure, monomeric syntaxin-1a and dodecylated (d)-SNAP-25 are separately reconstituted into proteoliposomes and subsequently assembled in the plane of merged target lipid bilayers. Examining single particle fusion between synaptobrevin-2 proteoliposomes and planar-supported bilayers containing the two different SNARE acceptor complexes revealed similar fast rates of fusion. Changing the stoichiometry of syntaxin-1a and d-SNAP-25 in the target bilayer had significant effects on docking, but little effect on the rates of synaptobrevin-2 proteoliposome fusion.

\subsection{Introduction}

The plasma membrane SNARE proteins syntaxin-1a and SNAP-25 can form unproductive 2:1 complexes that slow down the rate of synaptobrevin-2 binding (Fasshauer, et al., 1997) and account for the slow rates of traditional SNARE-mediated in vitro fusion assays (Schuette, et al., 2004; Weber, et al., 1998). Preventing the formation of a 2:1 complex by utilizing a 1:1:1 acceptor complex of syntaxin-1a, SNAP-25, and a short synaptobrevin-2 peptide (residues 4996), termed the $\Delta \mathrm{N}$ complex, greatly accelerates reconstituted fusion assays (Pobbati, et al., 2006) and was necessary in the initial SNARE mediated planar supported bilayer fusion assay that used the monolayer-fusion method to form the planar bilayer surface (Domanska, et al., 2009).

The $\Delta \mathrm{N}$ complex has been instrumental in studying membrane composition and curvature on fusion (Domanska, et al., 2009; Hernandez, et al., 2014; Kreutzberger, et al., 2015), but not 
resorting to an artificial SNARE complex when attempting to understand how fusion occurs in cells is of great interest. Traditionally, there are two methods for SNARE protein reconstitution. The first is when syntaxin-1a is purified in octyl- $\beta$-D-glucoside or sodium cholate and assembled into an acceptor complex with soluble SNAP-25a during co-expression in Escherichia coli or post-expression in cholate, 3-[(3-cholamidopropyl)di methylammonio]-1-propanesulfonate, or octyl- $\beta$-D-glucoside (CHAPS). Depending on input stoichiometry and other conditions, this results, to different degrees, in the formation of a 2:1 syntaxin-1a:SNAP-25a complex that does not readily bind to synaptobrevin-2 (Domanska, et al., 2009; Pobbati, et al., 2006). The oligomeric state of syntaxin-1a is dependent on the purification and assembly detergent and an exclusively monomeric syntaxin-1a can be produced in dodecylphosphocholine (DPC) (Liang, et al., 2013). Here two new procedures will be shown to form SNARE acceptor complexes capable of fast in vitro fusion. The first procedure, monomeric syntaxin-1a is mixed with soluble SNAP25 and purified as a complex by anionic exchange in DPC. The second procedure monomeric syntaxin-1 is reconstituted into proteoliposomes and mixed to form a planar-supported bilayer with liposomes containing quadrupally dodecylated SNAP-25 (d-SNAP-25), which then spontaneously assemble in the plane of the membrane to form a fusion-competent SNARE acceptor complex. 


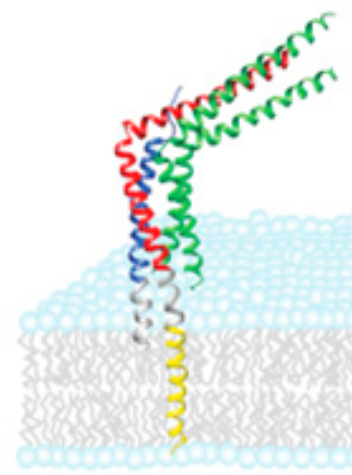

$\Delta \mathrm{N}$ complex

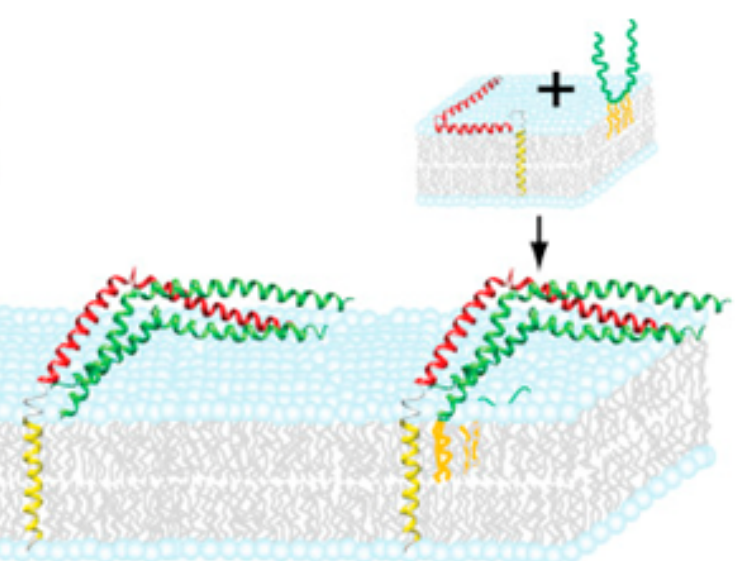

1:1 SyxH3:SN25 (DPC)
SyxH3:dSN25

Figure 4.1: Active plasma membrane SNARE acceptor complexes that exhibit fast reconstituted fusion are the $\Delta N$ complex, 1:1 syntaxin-1a:SNAP-25 purified by anionic exchange in the presence of DPC, and monomeric syntaxin-1a and d-SNAP-25 allowed to spontaneously assemble when formed into the planar supported bilayer. Figure adapted from (Kreutzberger, et al., 2016).

\subsection{Results}

Syntaxin-1a (residues 183-288) was prepared in DPC as previously described in Liang et al. (Liang, et al., 2013) which results in a monomeric syntaxin-1a while purification in CHAPS results in aggregation, Figure 4.2. Combining syntaxin-1a with soluble SNAP-25 in the presence of detergent followed by purification by anionic exchange results in a 1:1 complex in the presence of DPC and a 2:1 complex in the presence of CHAPS, Figure 4.3. Alternatively, we expressed wild-type SNAP-25 and used alkylation with dodecyl chains to the four native cysteines, Figure 4.4. This form of dodecyl-SNAP-25 (d-SNAP-25) closely resembles palmitoylated SNAP-25. SNARE acceptor complexes were formed between monomeric 
syntaxin-1a (purified in DPC) and d-SNAP-25 by letting them spontaneously assemble in the membrane.

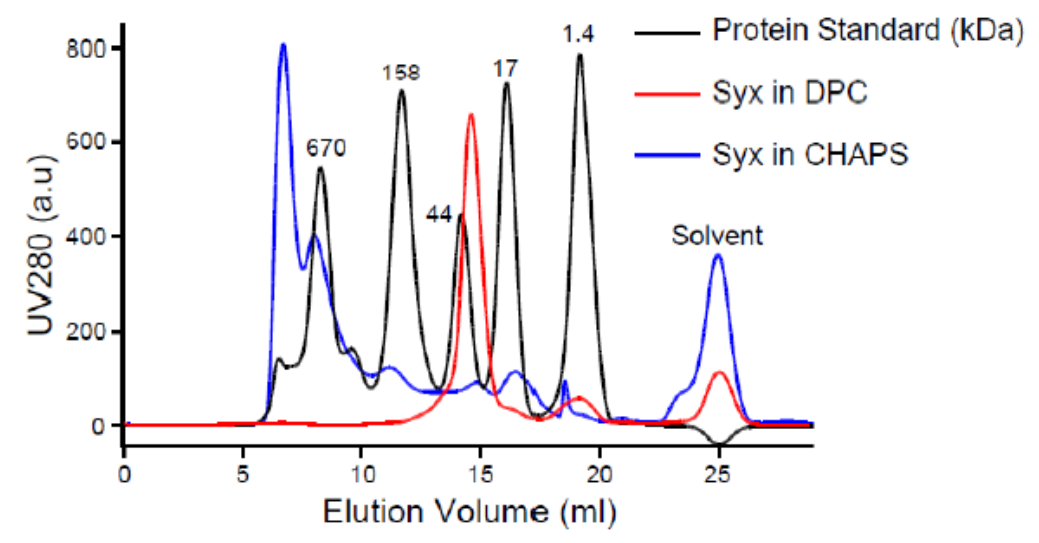

Figure 4.2: Size exclusion chromatography of syntaxin-1a purified in DPC and CHAPS indicating an oligomeric protein in CHAPS (blue) and a monomeric protein in DPC (red). The elution peak of syntaxin-1a in DPC corresponds to the $\sim 12 \mathrm{kDa}$ fragment of syntaxin-1a in a micelle with $\sim 70$ DPC molecules. The figure is adapted (Kreutzberger, et al., 2016; Liang, et al., 2013). 

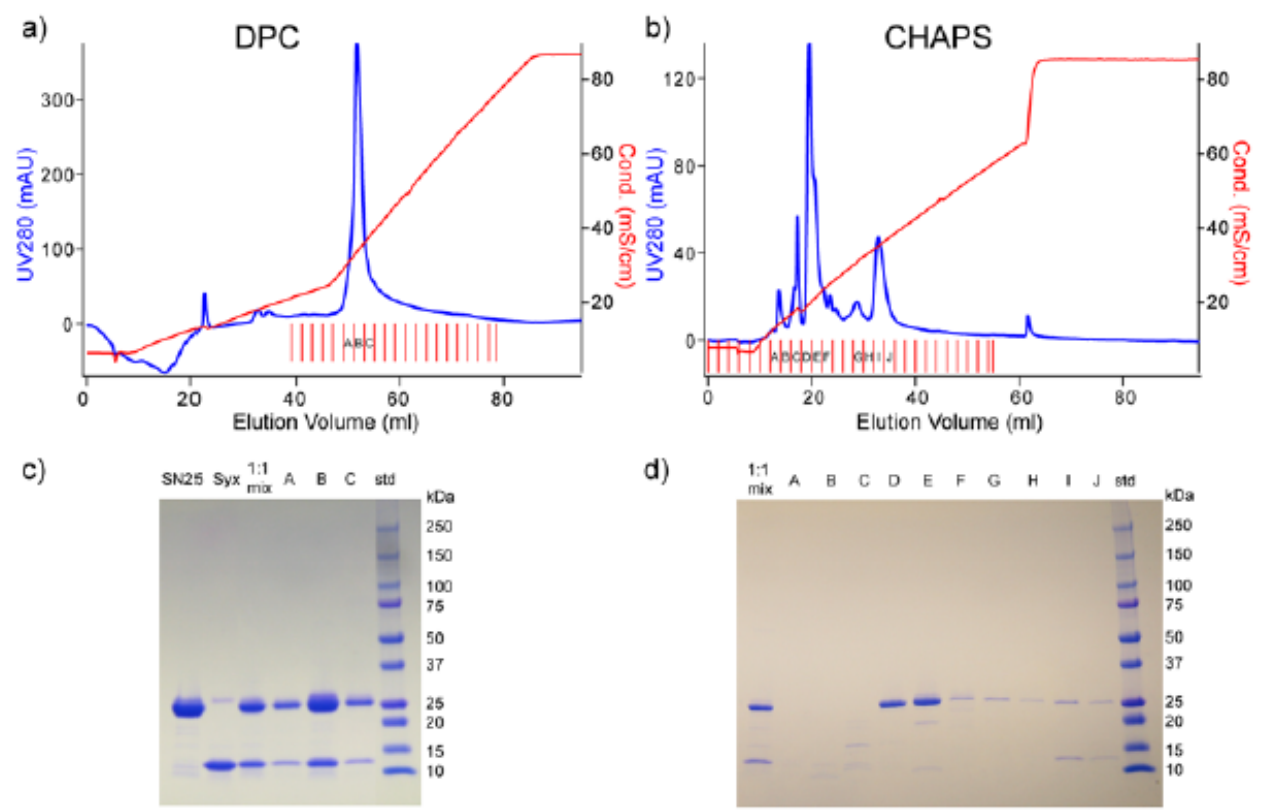

Figure 4.3: (a) MonoQ column elution profiles and SDS-PAGE gels demonstrating the formation of a 1:1 syntaxin-1a(183-288):SNAP-25 complex in DPC, (b) but 2:1 syntaxin-1a(183-288):SNAP-25 complex in CHAPS. The blue traces show UV absorptions (left axes) and red traces show the eluted buffer conductivities (right axes). The red perpendicular lines at the bottom denote collected fractions and the factions run on SDS-PAGE gels are labeled with corresponding capital letters. In these examples, fractions $A, B$, and $C$ in (a) and fractions $I$ and $J$ in (b) are the final purified complexes used in subsequent experiments. (c, d): SDS-PAGE gels of complexes purified in (c) DPC and (d) CHAPS. In both detergents, equal amounts of syntaxin-1a and SNAP-25 were mixed and incubated overnight before MonoQ purification. Since SNAP-25 is about twice the molecular mass of syntaxin-1a(183-288), the SNAP-25 band is twice as strong as the syntaxin-1a band when they are in molar ratio of 1:1 (c). However, in panel d (lanes $I$ and $J$ ) the syntaxin-1a and SNAP-25 bands exhibit about equal stain intensity, indicating the formation of a 2:1 syntaxin-1a:SNAP-25 complex in CHAPS. Figure taken from (Kreutzberger, et al., 2016). 
Bulk ensemble lipid mixing assays were used to compare 1:1 syntaxin-1a(183-288):SNAP-25 co-purified in DPC, 2:1 complex co-purified in CHAPS, the 1:1:1 syntaxin-1a(183-288):SNAP25:synaptobrevin-2(49-96) ( $\Delta \mathrm{N}$ complex), and co-reconstituted syntaxin-1a (183-288) and dSNAP-25. The 2:1 complex had substantially reduced rates of lipid mixing, while the rates of the 1:1 co-purified complex and the 1:1 syntaxin-1a:d-SNAP-25 co-reconstituted complex had lipid mixing that approached the $\Delta \mathrm{N}$ complex, Figure 4.5.
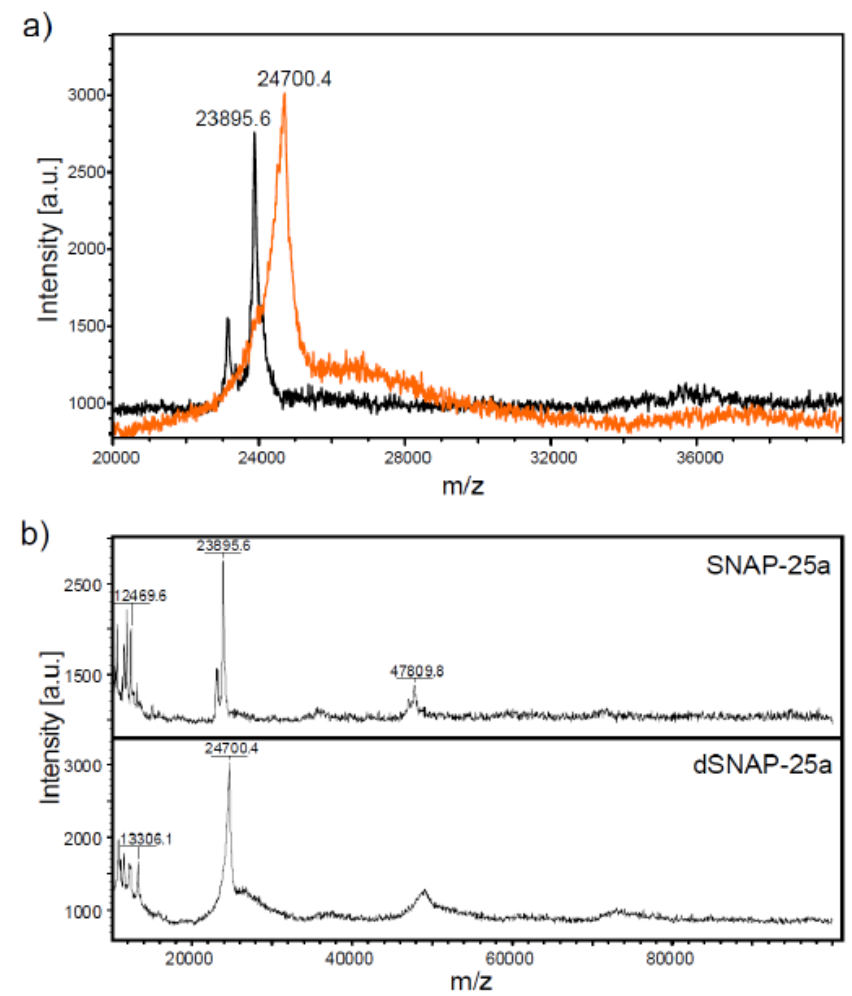

Figure 4.4: MALDI-TOF mass spectra of wild-type SNAP-25a and dodecylated SNAP-25a (dSNAP25a). (a) Spectral overlay of the 20-40kDa range of SNAP-25a (black) and dSNAP-25a (orange). (b) The whole spectral window as collected (10-100 kDa) for SNAP-25a (top) and dSNAP-25a (bottom). The theoretical molecular masses of unmodified and quadruple dodecylated SNAP-25a are 23907 and 24707, respectively. Mass-spec sample preparation was performed by Binyong Liang. This figure was adapted from (Kreutzberger, et al., 2016). 
The bulk assay reports the convolution of the docking and fusion kinetics without distinguishing between them (Hernandez, et al., 2014). The planar supported bilayer single vesicle fusion assay was used to separate the docking and fusion steps of the overall fusion reaction. The different acceptor complex assemblies were reconstituted in the planar bilayer using the monolayer fusion method to incorporate the acceptor complex into the bilayer when forming the outer leaflet of the membrane. The complex of syntaxin-1a and d-SNAP- 25 was formed by reconstituting them into separate proteoliposome and mixing these liposomes 1 to 1 when forming the planar bilayer and letting the complex spontaneously assemble in the membrane. Comparing the docking of synaptobrevin-2 proteoliposomes to different plasma membrane SNARE complexes revealed differences between them, Figure 4.6.

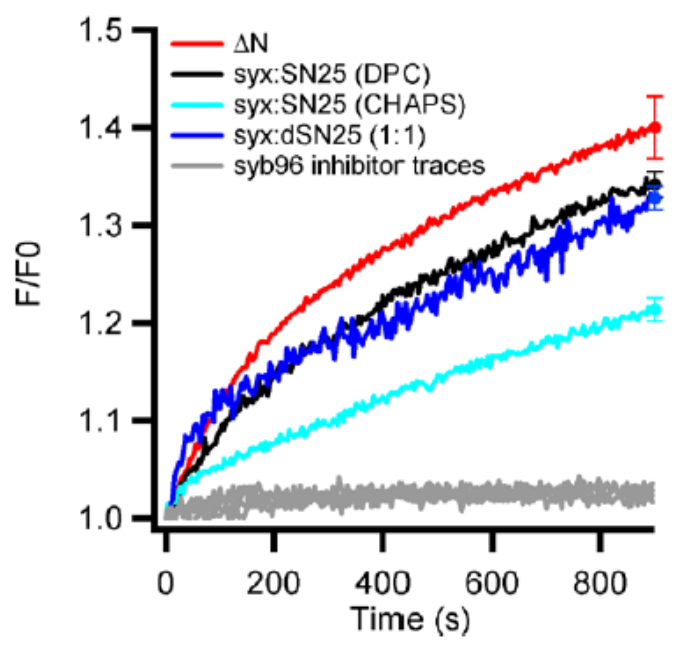

Figure 4.5: Bulk ensemble lipid mixing of synaptobrevin-2 proteoliposomes with proteoliposomes containing different SNARE acceptor complexes purified and assembled in different detergents: pre-assembled $\Delta \mathrm{N}$ complex (red), syntaxin-1a:SNAP-25 preassembled in DPC (black), and syntaxin-1a:SNAP-25 preassembled in CHAPS (cyan), and 1:1 syntaxin-1a:d-SNAP-25 assembled in the proteoliposome (blue). Each curve is an average trace of 4-6 lipid mixing experiments with error bars of the last point shown. Inhibition of each complex with a soluble synaptobrevin-2 peptide (residues 1-96) is shown in grey. This figure was adapted from (Kreutzberger, et al., 2016). 
a)

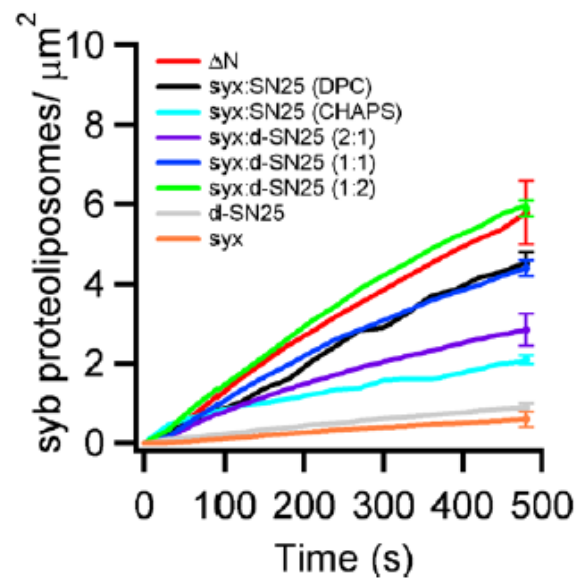

b)

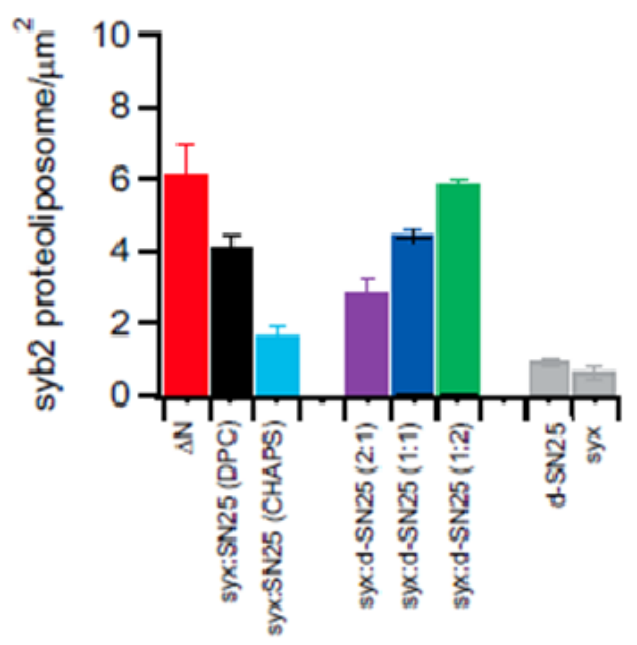

Figure 4.6: (a) Docking of synaptobrevin-2 proteoliposomes to different SNARE acceptor complexes or single SNAREs reconstituted in planar supported bilayers. Traces are averages of 3-6 bilayers with error bars at the end that represent the standard errors after 8 minutes of docking. (b) Saturation of docking of synaptobrevin-2 proteoliposomes to different SNARE acceptor complexes reconstituted in planar supported bilayers. This figure was adapted from (Kreutzberger, et al., 2016). 
a)

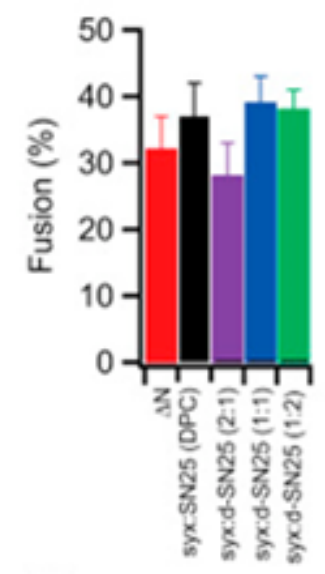

b)

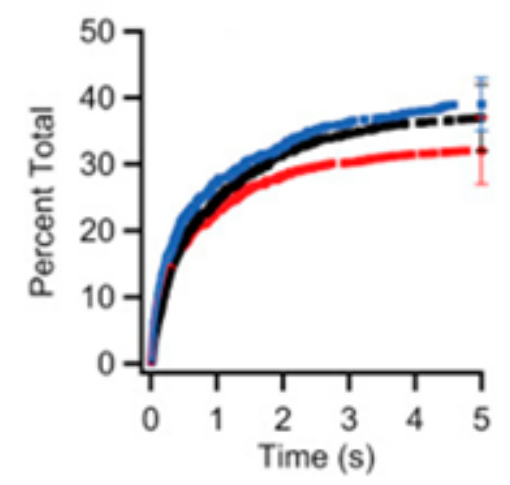

c)

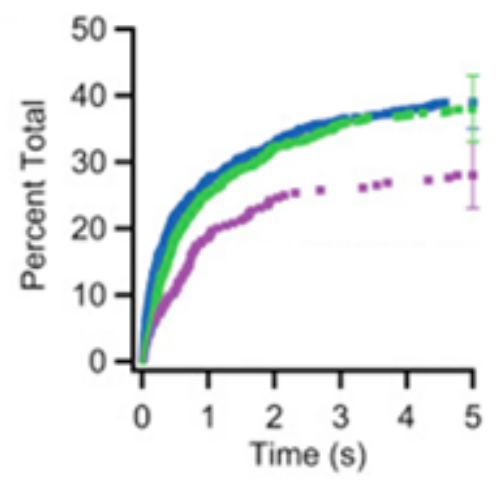

Figure 4.7: (a) Fusion efficiencies (\% fused of all docked) of synaptobrevin-2 proteoliposomes with different SNARE complexes. (b and c) Fusion kinetics of synaptobrevin-2 proteoliposomes with colors as indicated in bar graphs. The kinetic curves are cumulative distribution functions derived from hundreds of single vesicle fusion events. Statistics are given in Table 4.1. This figure was adapted from (Kreutzberger, et al., 2016).

\begin{tabular}{cccc}
\hline $\begin{array}{c}\text { SNARE acceptor } \\
\text { complex }\end{array}$ & Number of bilayers & Total number of events & Total fusion events \\
\hline$\Delta$ N & 9 & 1781 & 549 \\
syx:SN25 (DPC) & 10 & 1437 & 506 \\
syx:d-SN25 (2:1) & 4 & 134 & 46 \\
syx:d-SN25 (1:1) & 9 & 1135 & 442 \\
syx:d-SN25 (1:2) & 3 & 641 & 218 \\
\hline
\end{tabular}

Table 4.1: Summary of single liposome fusion events with planar supported bilayers containing different SNARE acceptor complexes. This table is adapted from (Kreutzberger, et al., 2016). 


\subsection{Discussion}

Millisecond timescale fusion of synaptobrevin-2 proteoliposomes was observed with SNARE acceptor complexes that no longer required a non-physiological peptide to ensure stoichiometric 1:1 syntaxin-1a:SNAP-25 acceptor complex formation. The efficiency and rate of fusion of the 1:1 syntaxin-1a:SNAP-25 and the 1:1 syntaxin-1a:dSNAP-25 complexes closely match those of the previously employed $\Delta \mathrm{N}$ acceptor complex. The use of DPC as the purifying and reconstitution detergent for syntaxin-1a appears to be the key ingredient to maintain the monomeric form of syntaxin-1a, presumably because of its tight association with DPC micelles (Liang, et al., 2013) . This form of syntaxin-1a can be assembled either with a soluble form of SNAP-25 in DPC or with a lipidated form of SNAP-25 in lipid bilayers. Although SNAP-25 is multiply palmitoylated in eukaryotic cells (Viet, et al., 1996), this posttranslational modification has not usually been included in previous attempts to reconstitute SNARE-mediated fusion in vitro. The exact number of palmitates that are attached to each SNAP- 25 may be less than four, and may vary depending on physiological conditions; our procedure quantitatively attaches four dodecyl chains, which, are shorter by four carbons than the native 16 carbon palmitates.

The similarity of the fusion efficacies and kinetics of all fusion competent complexes that were investigated in this study suggest that none of the preparations were limited by a minimal concentration of SNAREs in the membrane that is necessary for fusion (Hernandez, et al., 2014). The results also suggest that fusion operates at the intrinsic efficiency of this particular SNARE system, and that this is not controlled by any accessory proteins or calcium. The most significant differences between different SNARE acceptor complex preparations are observed in their different docking probabilities. These likely reflect the concentration of active SNARE 
complexes with a 1:1 syntaxin-1a:SNAP-25 stoichiometry and support the notion that previous reports of limited efficiencies of overall bulk fusion reactions are the result of inactive oligomeric syntaxin-1a products that are produced by the most common previous SNARE reconstitution procedures. The methodological advancer of SNARE acceptor complex preparation with the highest possible activities is a major advance that allows further investigation of SNARE proteins without the artificial syb49 peptide that was previously required to assemble a highly active complex. Subsequent work (in chapter 7 and 8) will demonstrate the use of this active 1:1 syntaxin-1:SNAP-25 complex as novel interaction with complexin-1 binding and that the addition of other accessory proteins with these SNARE complexes can be used to reconstitute a calcium dependent fusion process that mimics cellular exocytosis. 


\section{Chapter 5: The role of membrane curvature in}

\section{SNARE cooperativity}

Hernandez, J.M., A.J.B. Kreutzberger, V. Kiessling, L.K. Tamm, and R. Jahn (2014) Proc. Nat. Acad. Sci. U.S.A., 111, 12037-12042.

These experiments were performed in collaboration with Matias Hernandez from the laboratory of Reinhart Jahn in the Department of Neurobiology, Max Planck Institute for Biophysical Chemistry, Göttingen, Germany. Planar supported bilayer docking and fusion assays were performed by Alex Kreutzberger. Lipid mixing experiments and cooperativity model were designed and conducted by Matias Hernandez.

\subsection{Summary}

Whether and how SNAREs cooperate to mediate fusion has been a subject of intense study, with estimates ranging from a single SNARE complex to 15 . Here we show that there is no universally conserved number of SNARE complexes involved as revealed by our observation that this varies greatly depending on membrane curvature. When docking rates of small ( $\sim 40$ $\mathrm{nm})$ and large $(\sim 100 \mathrm{~nm})$ proteoliposomes reconstituted with different syb2 densities are taken into account, the lipid mixing efficiency was maximal with small liposomes with only one syb2, whereas 23-30 syb2 were necessary for efficient lipid mixing in large liposomes. Our results can be rationalized in terms of strong and weak cooperative coupling of SNARE complex assembly 
where each mode implicates different intermediate states of fusion that have been previously identified by electron microscopy (Hernandez, et al., 2012). The plasticity of SNAREs to engage in different coupling modes is an important feature of the biological ubiquitous SNAREmediated fusion reactions.

\subsection{Introduction}

The question of whether and how SNAREs cooperate to mediate fusion has received substantial attention. Although some studies have left open the possibility that the number of SNARE complexes that cooperate during fusion is variable (Domanska, et al., 2010; Mohrmann, et al., 2010), much attention has been given to the notion of a preferred number of SNARE complexes, with estimates varying from a single SNARE complex (van den Bogaart, et al., 2010) to 15 (Montecucco, et al., 2005), although more recent estimates vary between two and eight (Mohrmann, et al., 2010; Sinha, et al., 2011; Shi, et al., 2012; Karatekin, et al., 2010; Domanska, et al., 2009). Unfortunately, this large disparity in results has not been appropriately explained, and it remains unclear whether the differences are a result of inherent properties of the particular set of SNAREs involved or rather originate from the biophysical characteristics of the fusing vesicle.

Here we investigate how the density of SNAREs affects fusion of liposomes to obtain mechanistic information on cooperativity. This was done by varying the SNARE density of syb2. The formation of t-SNARE proteins into "off-pathway" complexes can compromise kinetic 
analysis (Fasshauer, et al., 2002; Xiao, et al., 2001) to avoid this the $\Delta \mathrm{N}$ complex was used in the following work (Pobbati, et al., 2006).

\subsection{Results}

Lipid mixing with SNARE liposomes (with the NBD/Rh FRET pair in the $\Delta \mathrm{N}$ membrane) was used to investigate fusion (Weber, et al., 1998; Chen, et al., 2006; Ji, et al., 2010). Content mixing assays were found to be too variable for the purpose of our strategy presented here. Regardless of the chosen fusion indicator, an often neglected aspect of these bulk assays is that they are rate-limited by docking (Smith \& Weisshaar, 2011). Observed changes in the kinetics of lipid mixing may have been misleadingly attributed to fusion when they actually were the result of preceding docking steps.

One way around this is to accelerate the rate of docking and thus enable the kinetics of lipid mixing to directly report the effect that SNARE density has on fusion. In principle, this could be done by exploiting the bimolecular dependence of liposome concentration on the rate of docking and increase its concentration. However, many studies already use high amounts of liposomes with lipid concentrations in the 0.1-3 mM range (Weber, et al., 1998; Ji, et al., 2010; Shen, et al., 2007; Parlati, et al., 1999; Ma, et al., 2013) suggesting that slow docking is mainly the result of the inefficiency of the assembly of the first trans SNARE complex. To overcome this, SNARE complex formation can be enhanced by using the $\Delta \mathrm{N}$ complex, which accelerates this process by at least 30-fold (when compared to traditional methods of t-SNARE purification) (Pobbati, et al., 
2006). In a dilute liposome regime (0.02-0.1 $\mathrm{mM}$ total lipid) we have previously found that with the $\Delta \mathrm{N}$ complex the rate of docking is accelerated to an extent similar to the rate of fusion, a kinetic condition known as partial-rate limiting (Hernandez, et al., 2012; Cypionka, et al., 2009).

Based on the above considerations, we designed experiments to measure the cooperativity of the actual fusion step in liposome-based lipid mixing assays. Our approach is centered on the principle of leveled docking rates across a series of reactions with different SNARE densities under partial rate-limiting conditions, thus allowing both docking and fusion to be kinetically resolved. To introduce this approach, we consider the rate equation for the generation of docked liposome [D]:

$$
\frac{d[D]}{d t}=k_{d}(s y b, \Delta N)\left[L_{s y b}\right]\left[L_{\Delta N}\right]
$$

where $\left[L_{\mathrm{syb}}\right]$ and $\left[L_{\Delta N}\right]$ are the concentrations of syb and $\Delta \mathrm{N}$ complex liposomes, respectively, and $k_{d}(s y b, \Delta N)$ is the bimolecular docking constant, which is a function of both synaptobrevin2 and $\Delta \mathrm{N}$ complex density. To exclude that docking itself is not cooperative with regard to SNARE density, we monitored docking using the planar supported bilayer docking assay with proteoliposomes of different syb2 densities, Figure 5.1A. This revealed a linear, noncooperative relation between the initial docking rate and SNARE density, Figure 5.1B. Having established non-cooperative docking, Eq. 1 states that a decreased rate of docking resulting from a reduced SNARE density [a lower $\mathrm{k}_{\mathrm{d}}(\mathrm{syb}, \Delta \mathrm{N})$ ] can be compensated by proportionally increasing the concentration of liposomes (a higher product of $\left[\mathrm{L}_{\mathrm{syb}}\right]\left[\mathrm{L}_{\Delta \mathrm{N}}\right]$ ), thus leveling the original docking rate. Although it may initially seem counterintuitive, an analysis of Eq. 5.1 shows that the amount by which concentrations of individual SNARE-liposomes should be 
increased to level docking rates is proportional to the change in $\mathrm{k}_{\mathrm{d}}(\mathrm{syb}, \Delta \mathrm{N})$. If a new rate constant $\mathrm{k}_{\mathrm{d}}(\mathrm{syb}, \Delta \mathrm{N})$, which is altered by a change in SNARE density on one of the SNAREliposomes by $\mathrm{n}$, where $\mathrm{n}$ is any real number greater than zero. We relate this new rate constant to the reference rate constant $\mathrm{k}_{\mathrm{d}}(\mathrm{syb}, \Delta \mathrm{N})$ by

$$
k_{d}(s y b, \Delta N)=n \cdot k_{d}^{\prime}(\operatorname{syb}, \Delta N)
$$



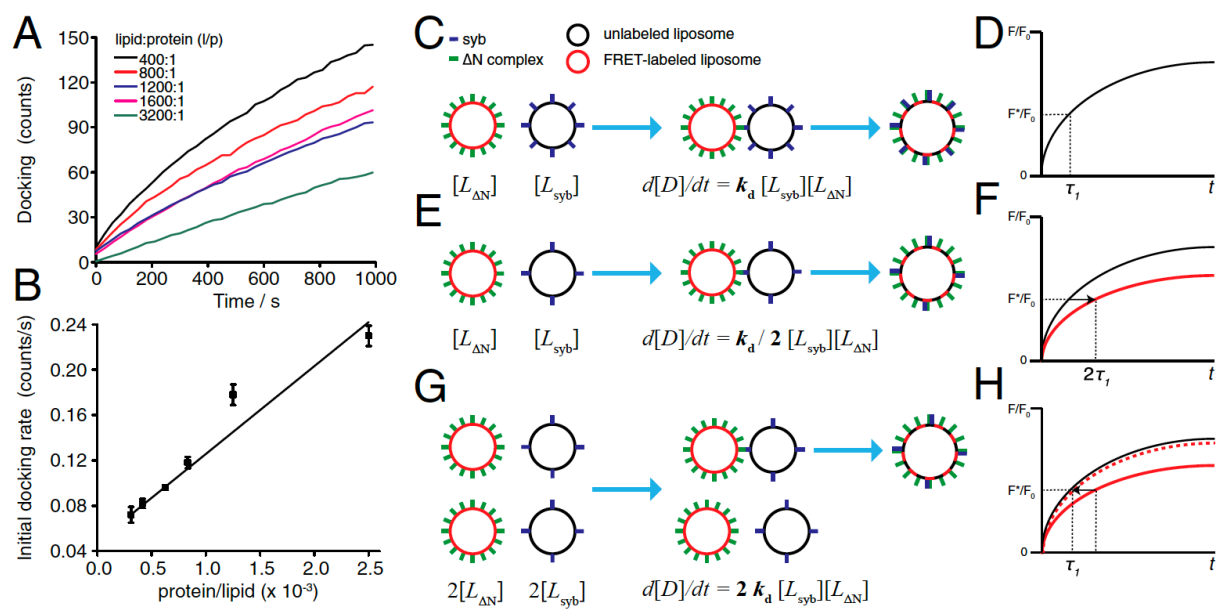

Figure 5.1: Schematic explanation of the principle of leveling docking for investigating SNAREmediated cooperativity in fusion. (A) Small $(\sim 40 \mathrm{~nm})$ proteoliposomes labeled with rhodamineDOPE and containing syb2 at the indicated densities were added to a planar supported bilayer reconstituted with the $\Delta \mathrm{N}$ complex (lipid:protein of 1000). Docking was measured by the increase in total fluorescence intensities (counts) using the planar supported bilayer. Lines of best fit are presented. (B) Initial docking rates obtained by TIRF microscopy in A as a function of synaptbrevin-2 density, showing that docking is non-cooperative within the range of lipid:protein of 400 to 3,200. Solid line: line of best fit (adjusted $R^{2}=0.94$ ). Data are rates from fits of docking traces in A with error bars originating from fitting function. (C) Liposomes dock with a rate that depends on the concentrations of $\Delta \mathrm{N}$ complex and synaptobrevin-2 liposomes $\left(\left[\mathrm{L}_{\Delta \mathrm{N}}\right]\right.$ and $\left[\mathrm{L}_{\mathrm{syb}}\right]$, respectively), as well as a kinetic constant $k_{d}$ that itself depends on SNARE density. (D) If the total reaction rate is limited by docking, the recorded fluorescence signal $F * / F_{0}$ for lipid mixing increases with the docking time constant $\tau_{1}$ (schematic trace shown). (E) Reducing the synaptobrevin-2 density by one-half will also reduce the docking rate by one-half of its original value. (F) Given that the docking reaction is still rate-limiting, the fluorescence signal $F^{*} / F_{0}$ now rises with a time constant of $2 \tau_{1}$, slowing down lipid mixing as schematically depicted in red. (G) This slowing down can be reversed by doubling both liposome concentrations (assuming halving the density does not affect fusion). The docking rate is now twice as fast as in $\mathrm{C}$; however, because there are twice as many liposomes, the relative rate is now leveled to the original docking rate in $\mathrm{C}$. (H) Thus, the relative lipid mixing normalized to the initial fluorescence $F_{0}$ is unaffected, reestablishing the signal $F^{*} / F_{0}$ to the reference trace (schematically shown as a dashed red trace). The pattern of reducing synaptobrevin-2 density while increasing both liposome concentrations is continued iteratively until lipid mixing is no longer recovered from leveling docking rates, which would indicate reduced cooperativity with respect to fusion. Kinetic model was created by Matias Hernandez. Figure is adapted from (Hernandez, et al., 2014). 
If liposome concentrations remain constant, the new docking rate will change by a factor of $n$ according to the rate equation

$$
\frac{d[D]}{d t}=n \cdot k_{d}^{\prime}(s y b, \Delta N)\left[L_{s y b}\right]\left[L_{\Delta N}\right]
$$

The goal is to find a way to prevent the change in the docking rate as a result of variations in the docking efficiency [i.e., changes in $k_{d}(s y b, \Delta N)$ ]. According to eq. 5.1 and eq. 5.3, this can be done by altering the concentration of one or both sets of liposome $\mathrm{L}_{\mathrm{syb}}$ and $\mathrm{L}_{\Delta \mathrm{N}}$ in a way that counteracts the change in $k_{d}(s y b, \Delta N)$. To test this formally, we introduced the following variable substitutions so that both liposome concentrations (and correspondingly the concentration of docked liposomes) are changed simultaneously by a factor of $1 / \mathrm{n}$ :

$$
\begin{aligned}
& {\left[L_{s y b}\right]=\left[L_{s y b}^{\prime}\right] / n} \\
& {\left[L_{\Delta N}\right]=\left[L_{\Delta N}^{\prime}\right] / n} \\
& {[D]=\left[D^{\prime}\right] / n}
\end{aligned}
$$

Combining and rearranging equations. 5.3-5.6 the following rate equation is obtained:

$$
\frac{d\left[D_{\prime}\right]}{d t}=k_{d}(s y b, \Delta N)\left[L_{s y b}^{\prime}\right]\left[L_{\Delta N}^{\prime}\right]=\frac{d[D]}{d t} / n
$$

Although the original and new docking rates differ by a factor of $1 / \mathrm{n}$, eq. 5.7 has the exact form of that of the reference rate eq. 5.1. In relative terms (i.e., normalized to $1 / n$ ), it can be seen that a loss (or gain) in docking efficiency can be counter balanced by proportionally increasing (or decreasing) the concentration of both liposomes Therefore, all lipid mixing traces were normalized to their initial fluorescence value $\mathrm{F}_{0}$ to allow quantitative comparison of the different density reactions. Simply speaking, this means that if the density of syb2 is halved the concentrations of both liposomes need to be doubled to compensate for the change in liposome docking. Therefore, any observed changes in lipid mixing in docking leveled reactions will indicate differences in fusion. 
Using this principle of leveled docking rates, we prepared a set of synaptobrevin-2 liposomes over a wide range of synaptobrevin- 2 densities and let them fuse with liposomes containing a fixed and excess amount of $\Delta \mathrm{N}$ complex as schematically portrayed in Figure 5.1C-H. Because $\Delta \mathrm{N}$ complex is in excess, we will assume for now that all synaptobrevin-2s within the vicinity of the first SNARE complex formed will readily find a binding partner and will initiate trans SNARE complex assembly.

Small proteoliposomes were prepared with four different syb2 densities ranging from a lipid/protein ratio of 400:1-3,200:1 and $\Delta \mathrm{N}$ complex in relative excess (lipid/protein $=300: 1)$. We note that these densities are nominal and that the orientation of reconstituted synaptobrevin-2 is random whereas $\Delta \mathrm{N}$ complex is predominantly right side out (van den Bogaart, et al., 2010). Regardless of the absolute effective concentrations, it is essential that the synaptobrevin- 2 concentrations are linearly scaled whereas the liposome concentrations of the different preparations are constant to quantitatively level docking rates. Quantification of syb2 by Western blot and of membrane lipid by assaying for liposome phosphate content confirm these requirements, Figure 5.2A. We then measured lipid mixing in bulk for all densities as done conventionally by maintaining liposome concentrations unchanged. As expected, the lipid mixing levels decreased as the syb2 density was reduced, Figure 2B, and the rate of lipid mixing was slowed as revealed by normalizing the traces to the final value of the reference reaction, Figure 2C. 

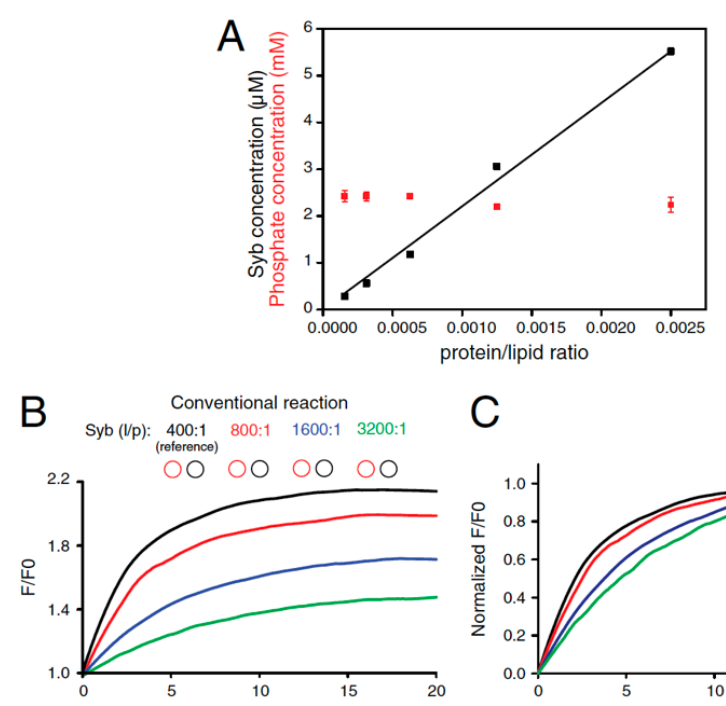

C
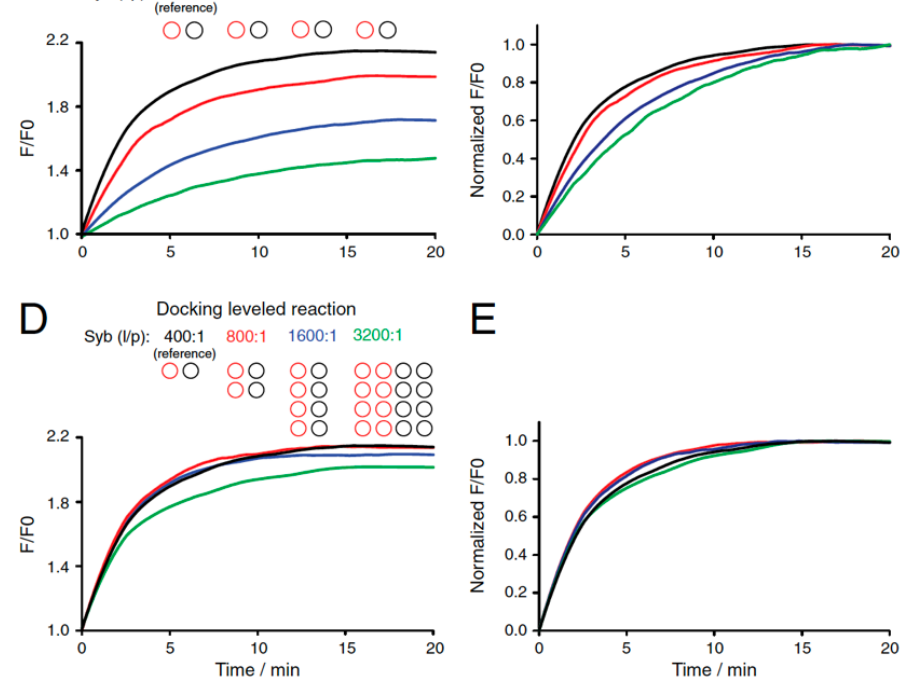

Figure 5.2: Leveled docking on small $(40 \mathrm{~nm})$ SNARE liposomes reveals negligible effect of SNARE density on lipid mixing. (A) A series of small synaptobrevin-2 liposomes were prepared at different synaptobrevin-2 densities and their synaptobrevin-2 protein (black) and phospholipid (red) contents were quantified by Western blot and organic phosphate determination (Rouser, et al., 1970) (B) Four small liposomes (unlabeled, black circles) with the depicted synaptobrevin-2 densities were mixed to $\Delta \mathrm{N}$ complex liposomes (FRET-labeled, red circles, $1 / \mathbf{p}=300: 1$ ) at constant liposome concentration (conventional assay), showing a decline of lipid mixing as the synaptobrevin-2 density was decreased.. (C) Same as $B$ but normalized to the final $F / F_{0}$ of the reference (black) trace after $20 \mathrm{~min}$, showing that the lipid mixing rate is also reduced. (D) When liposome concentrations are increased in the relative amounts depicted, the leveling of docking causes almost all lipid mixing traces to converge to the reference level, indicating no effect of density on fusion. Only at nominal $1 / p=3,200: 1$ is lipid mixing not fully recovered, suggesting a minor subpopulation of liposomes not having enough SNAREs for efficient fusion begins to emerge. (E) Normalization of traces in $D$ to the final $F / F_{0}$ of the reference trace (black) after 20 min reveals that the lipid mixing speeds are essentially identical. Data was collected by Matias Hernandez. Figure is adapted from (Hernandez, et al., 2014). 
When the same liposomes were mixed at increased leveled concentrations while SNARE densities were reduced, we observed the lipid mixing trace of the syb2 800:1 liposomes converge to the reference reaction of the syb2 400:1 liposomes, Figure 2D. Thus, halving the syb2 density had no effect on lipid mixing, a behavior we confirmed with fusion measurements of single syb2 liposomes in the planar supported bilayer fusion assay, Figure 5.3. Similarly, a fourfold reduction of syb2 density also had no effect on the efficiency of lipid mixing, further suggesting the reduction in lipid mixing in the conventional, not docking-leveled reaction (Figure 2B) is due to an effect on docking. However, when the syb2 density was reduced eightfold to lipid/protein $=3,200: 1$, we observed a slight decline in efficiency as inferred from a partial and incomplete recovery of lipid mixing to the reference level, Figure 2D. Interestingly, the lipid mixing speeds were the same for all reactions once docking rates were leveled, suggesting that efficiency but not speed begins to decrease at lipid/protein 3,200:1, Figure 2E.
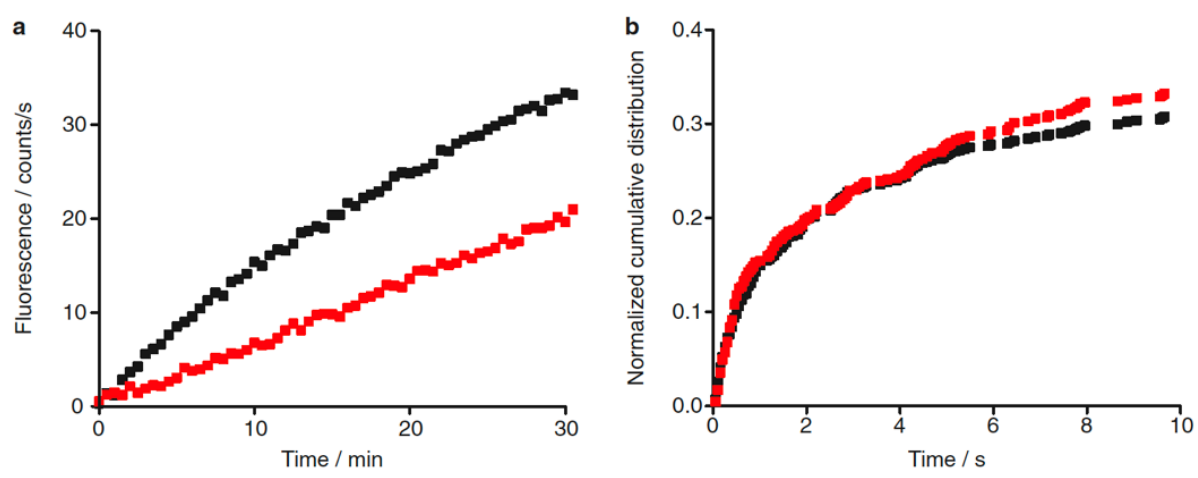

Figure 5.3: Single liposome fusion events measured by TIRF microscopy confirm no effect on fusion when the synaptobrevin-2 density is halved. (A) Docking kinetics of a small synaptobrevin-2 liposome with $1 / p=300: 1$ (black) and $1 / p=600: 1$ (red) to a planar supported bilayer reconstituted with $\Delta \mathrm{N}$ complex $(1 / p=1,000: 1)$ determine by total fluorescence using TIRF microscopy. As expected, the docking rate is reduced at a lower $1 / p$ ratio. (B) Comparative cumulative distributions of single liposome fusion events to a planar supported bilayer using TIRF of the same small liposomes in A. The kinetics of fusion are essentially the same. Figure is adapted from (Hernandez, et al., 2014). 
We next asked whether membrane curvature stress has an impact on the effect of SNARE density on lipid mixing. We prepared large ( $>80-\mathrm{nm}$ diameter) liposomes that have approximately twofold less curvature than the small liposomes used previously (Hernandez, et al., 2012). To rigorously compared the effect of curvature on fusion, we first recorded single fusion events in the planar supported bilayer fusion assay with small and large syb2 liposomes. This allowed direct evaluation of the effect of curvature on fusion. Figure 5.4A shows that large syb2 liposomes had a lower efficiency (two- to threefold) and speed (approximately six fold) than small syb2 liposomes with similar lipid/protein ratios. Encouraged by this results, we conducted an extensive analysis of bulk liposomes fusion measurements with large liposomes with different SNARE densities, similar to those described with small SNARE liposomes.

As with small liposomes, the syb2 concentrations scaled linearly with density and the lipid phosphate concentrations remained constant over a wide range of SNARE densities, Figure 5.4B. Liposome lipid mixing reactions performed in the conventional way (Figure 5.4C, D) and with leveled docking rates (Figure 5.4E, F) show that the lipid mixing levels were enhanced by increased syb2 density, but almost all cases the enhancement did not restore lipid mixing to the reference level of syb2 400:1. This shows that the relation between lipid mixing and SNARE density is distinct, with these larger liposomes and that SNARE densities become more limiting at much higher densities. 

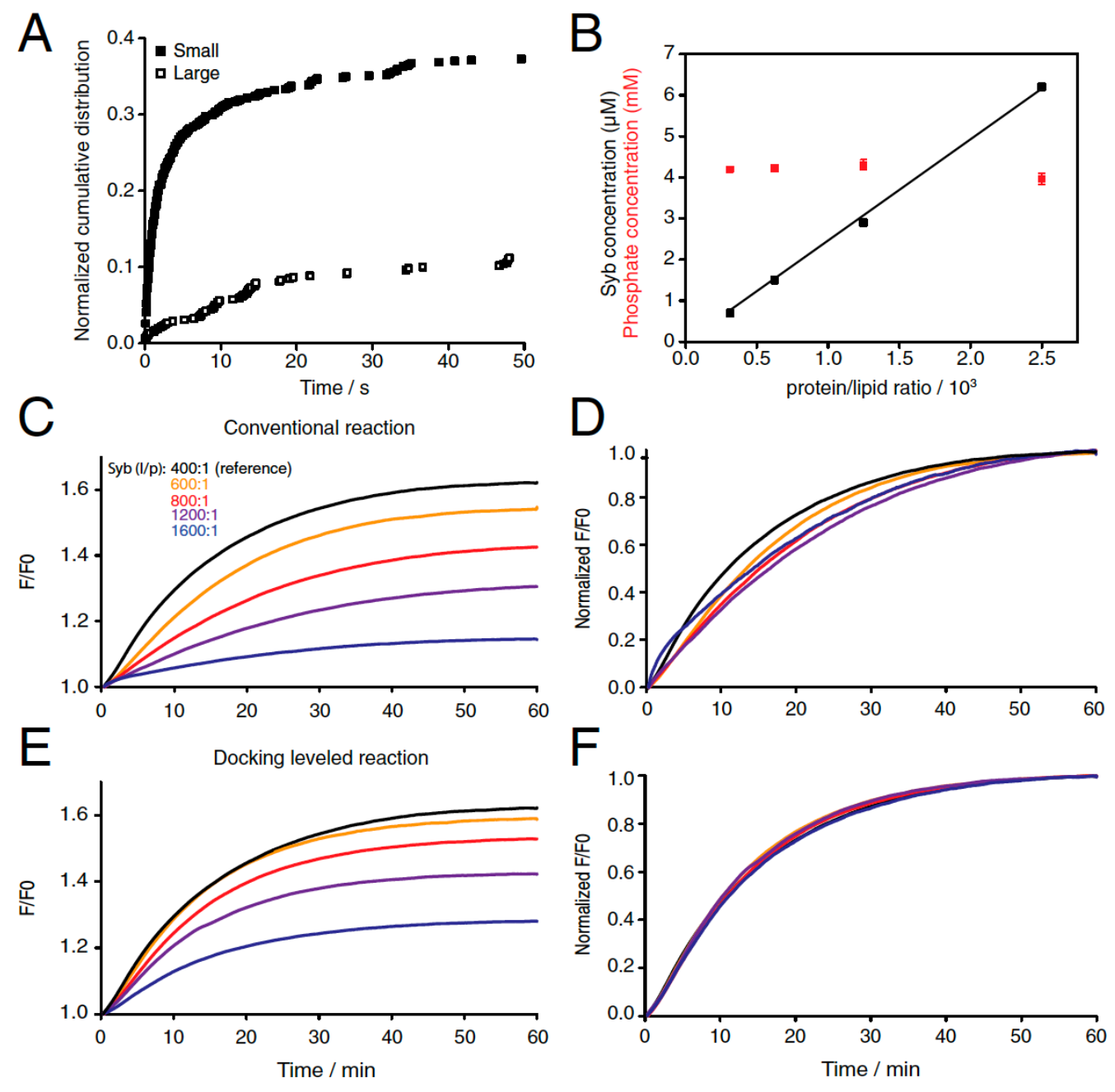

Figure 5.4: Lipid mixing on large ( $\sim 90 \mathrm{~nm})$ SNARE liposomes is more sensitive to changes in SNARE density, indicating a higher requirement for SNARE cooperativity. (A) Cumulative distribution function obtained by TIRF microscopy of single fusion events showing small liposomes fuse more efficiently and rapidly than large synaptobrevin-2 liposomes (both at nominal $1 / \mathbf{p}=$ 300:1) with a supported planar bilayer containing $\Delta N$ complex at $1 / p=1,000: 1$. Lipid compositions of liposomes used for TIRF microscopy were quantitatively different from those used for bulk experiments. (B) Synaptobrevin-2 protein concentration measured by Western blot (black) and phospholipid concentration (red) of four large liposome samples between the nominal ranges $1 / \mathbf{p}=$ 400:1 and 3,200:1. (C) Lipid mixing of large labeled $\Delta N$ complex liposomes $(1 / p=300: 1)$ reacted with large synaptobrevin-2 liposomes at five different SNARE densities at constant liposomes concentration (conventional assay). (D) Normalization of lipid mixing traces from $\mathrm{C}$ to the final $F / F_{0}$ after 60 min of the reference reaction (black). (E) Synapotbrevin-2 and $\Delta \mathrm{N}$ complex liposome were reacted at same protein densities as in $\mathrm{C}$, but liposome concentrations were increased proportionally to level docking rates revealing a partial recovery of lipid mixing. (F) The same traces as in $\mathbf{E}$ normalized to the final $F / F_{0}$ after 60 minutes of the reference trace (black), indicating that the lipid mixing speeds are identical after docking is leveled. Data in panels B-F were collected by Matias Hernandez. Figure is adapted from (Hernandez, et al., 2014). 
We repeated lipid mixing measurements for both small and large SNARE liposomes and plotted the final lipid mixing levels normalized to the lipid mixing of the reference density on a syb2/lipid and synaptobrevin-2/liposome basis (Figure 5.5 A, B). The number of synaptobrevin2 per liposome was calculated by taking the average diameters from representative small and large liposomes samples previously determined by field-flow-fractionation coupled to multiangle laser light scattering (FFF-MALLS) (Hernandez, et al., 2012). These were 40 and $90 \mathrm{~nm}$ for small and large liposomes, respectively, and were used to estimate the number of synaptobrevin-2s per liposome in Figure 5.5B. To calculate the number of lipids per liposome, we used the formula

$$
\frac{V_{\text {outer }}-V_{\text {inner }}}{L_{V}}=\frac{\frac{4}{3} \pi\left(r^{3}-(r-b)^{3}\right)}{L_{V}}
$$

where $\mathrm{V}_{\text {outer }}$ is the spherical volume defined by the outer radius of the liposome, $\mathrm{V}_{\text {inner }}$ is the spherical volume defined by the inner radius, $\mathrm{r}$ is the liposome radii, $\mathrm{b}$ is the bilayer thickness, and $\mathrm{L}_{\mathrm{v}}$ is the molecular volume of a lipid. Unless stated otherwise, we assumed a membrane bilayer thickness of $4 \mathrm{~nm}$ and used a molecular volume of a lipid of $1.25 \mathrm{~nm}^{3}$, which is close to the volume of 1-palmitoyl-2oleoyl-sn-glycero-3-phosphocholine in a membrane containing 10\% cholesterol at $30^{\circ} \mathrm{C}$ (Greenwood, et al., 2006). With the number of total lipids and the corrected number of synaptobrevin-2 per lipid from Figure 5.5A, we can determine the average number of synaptobrevinj-2 per liposome via the following calculation:

$$
\frac{s y b}{\text { liposome }}=\frac{s y b}{\text { lipid }} \times \frac{\frac{4}{3} \pi\left(r^{3}-(r-b)^{3}\right)}{L_{V}}
$$

These plots reveal the critical SNARE densities in both types of liposomes, below which the reactions can no longer be docking- rate leveled (i.e., the thresholds where SNARE densities 
become limiting for the fusion step in the overall reaction). The values in these plots are all corrected for total lipid and protein concentrations as well as for the random orientation of syb2 on the liposomes. Therefore, syb2/lipid or liposome values no longer match exactly between small and large liposomes. For both liposome sizes, we noted two clearly distinguishable regions: a constant region at the higher SNARE densities where data points align closely to the dashed red line representing full lipid mixing efficiency and where lipid mixing is unaffected by changes in SNARE density and a sharply increasing linear region where lipid mixing decreases with increasing SNARE density up to the full efficiency level. The intersection between the two lines corresponds to the SNARE density below which lipid mixing efficiency is compromised. We refer to it as the lipid mixing efficiency threshold.

We observed that large liposomes require 1.5-3 times more synaptobrevin-2 per lipid, that is, about 1 syb2 per 2,000 $\mathrm{nm}^{2}$ or 500 synaptobrevin-2 per square micrometer, to reach the lipid mixing level of small liposomes. This is evident from the differences in both the slope of the linear regions and the lipid mixing efficiency threshold. When the same comparison is made on a synaptobrevin-2-per-liposome basis, the differences are even greater: Approximately three synaptobrevin-2 are required to reach maximal lipid mixing efficiency of small liposomes, but 23-30 synaptobrevin-2 are required to reach this threshold with large liposomes (Figure 5.5B). These results strongly suggest that a greater number of SNAREs is needed to overcome the higher energy barrier for efficient fusion of the larger liposomes. 

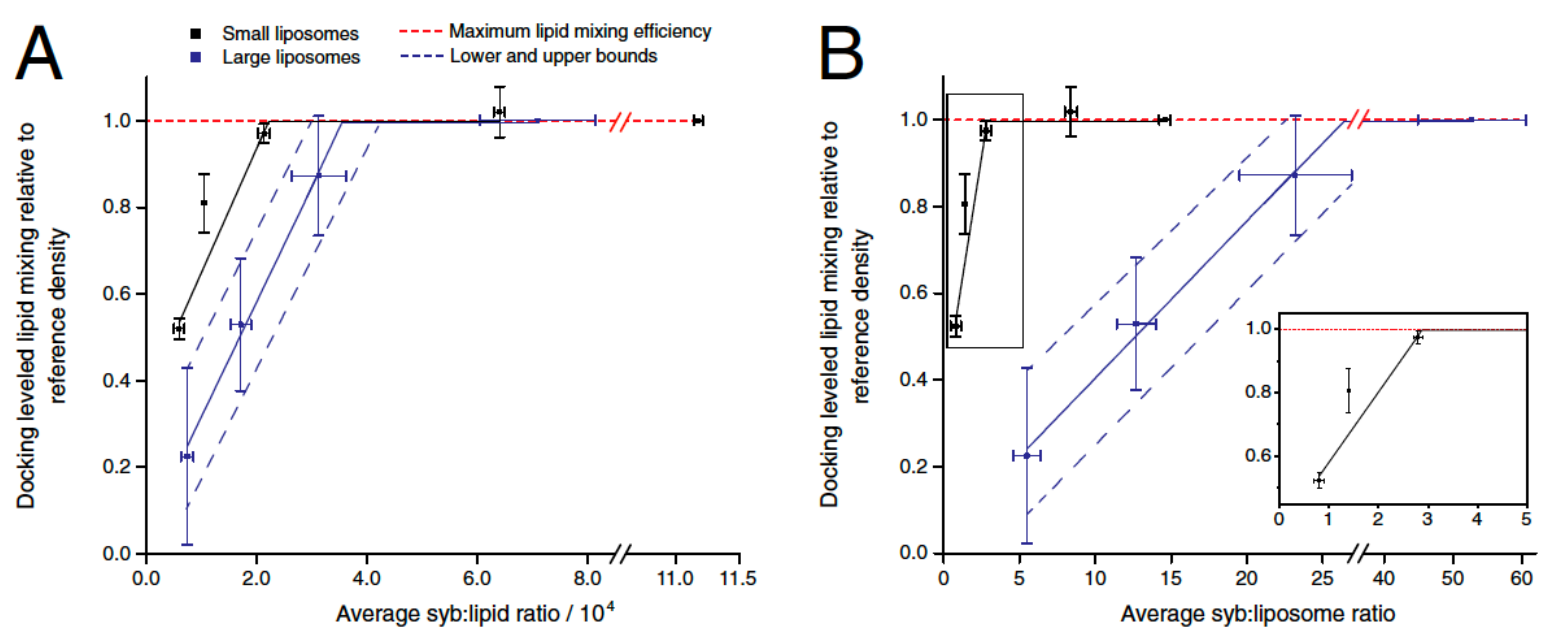

Figure 5.5: Quantitative comparison of lipid mixing of small and large liposomes provides insights on SNARE cooperativity. (A) The final lipid mixing value of all reactions normalize to the maximum lipid mixing efficiency (red dashed line) is plotted versus the corrected synaptobrevin:lipid ratio (densities were converted from the nominal $1 / p$ and from independently measured synaptobrevin-2 concentrations and phospholipid contents). Lines of best fit (solid lines) are shown for linearly increasing lipid mixing regions, with the intersection between the linear fit and the maximum efficiency (dashed horizontal line) denoting the lipid mixing threshold, below which fusion efficiency begins to be suboptimal. The threshold for large liposomes was treated as a range given the relatively high variability, with the dashed blue lines marking approximate upper and lower bounds. (B) The data of A replotted on a per-liposome basis to evaluate how lipid mixing varies when the total numbers of synaptobrevin-2 per liposomes are taken into account. For calculation, we assumed a bilayer thickness of $4 \mathrm{~nm}$ and diameter of $40 \mathrm{~nm}$ for small liposomes and $90 \mathrm{~nm}$ for large liposomes obtained by light scattering (Hernandez, et al., 2012). (Inset) A close-up view of the marked rectangular area showing the lipid mixing efficiency threshold on small liposomes. Ordinate error bars represent SDs from three to four independent experiments, and abscissa error bars represent SDs from two to three organic phosphate determinations. Synaptobrevin-2 protein concentrations were measured by quantitative Western blot from one full series of SNARE liposomes and divided by 2 to account for the random orientation of synaptobrevin-2 across the membrane (Hernandez, et al., 2012; van den Bogaart, et al., 2010). Data was collected by Matias Hernandez. Figure is adapted from (Hernandez, et al., 2014). 
Our results so far uncover a substantial difference in the ability of SNAREs to lipid mixing on small and large liposomes, a heavier we attribute to the rapid and nonlinear reduction in curvature stress as liposome size is decreased (Hernandez, et al., 2012). Still, we did not anticipate the curvature dependency of SNARE density threshold for efficient lipid mixing to be so large. How many SNAREs are then required to fuse small liposomes? Although at first sight the threshold would seem to suggest three (Figure 5.5A), it is important to note that this is an average. SNAREs are distributed on liposomes with a random poisson distribution, a feature that becomes more important to consider at very low SNARE densities (van den Bogaart, et al., 2010). It has been shown that on small liposomes with a lipid/protein ratio of $8,000: 1$ and 16,000:1 (when taking into account externally orientated synaptobrevin-2) $25 \%$ and $45 \%$ of liposomes are without any synaptobrevin-2, respectively. This correlates well with our observation that at a corrected lipid/protein of 9,500:1 and 17,000:1 (Figure 5.5A) the lipid mixing amount is $80 \%$ and $50 \%$ of the maximum, respectively. Thus, it is likely that the decrease in lipid mixing on small liposomes is not due to a diminishing cooperative effect as the SNARE density is reduced, but simply to the appearance of liposomes depleted of SNAREs. This strongly supports the conclusion that, at least for small liposomes, one SNARE complex is necessary and sufficient for fusion and is therefore non-cooperative (van den Bogaart, et al., 2010).

To evaluate whether SNARE depletion also affects the threshold on large liposomes, we simulated a random distribution of synaptobrevin-2 and found that the population of large liposomes without any synaptobrevin-2 is negligible at the lipid mixing efficiency threshold. 
Therefore, it cannot explain the drop in lipid mixing efficiency, Figure 5.6. Because the lipid mixing threshold occurs at around 23-30 synaptobrevin-2 per liposome (assuming an average diameter of $90 \mathrm{~nm}$ ), the question arises as to whether these synaptobrevin-2 are already locally present at initial membrane contact (either instantaneously or recruited by rapid diffusion before fusion is first observed (Bacia, et al., 2004)) or whether they are dispersed over a greater contact area. To gain more insight as to what may be occurring upon membrane contact, we constructed a simple geometric model to estimate the number of neighboring synaptobrevin- 2 that are present and within reach at the time when the first trans SNARE complex is formed Figure 5.7. To estimate the number of SNARE complexes at the docking interface, we considered the situation when the first SNARE complex nucleates at the $\mathrm{N}$ terminus, allowing docking between two membranes. aWe asked how many additional SNARE complexes would be readily available for SNARE complex formation in the vicinity of the first SNARE complex formed. Because in our experiments $\Delta \mathrm{N}$ complex is in excess, we make the assumption that any syb2 within this vicinity will readily form a complex. The determining factor of whether neighboring synapborevin-2 can engage in complex assembly is $h$, the minimum distance between opposing membranes needed for the $\mathrm{N}$ termini of synaptobrevin- 2 and $\Delta \mathrm{N}$ complex to be able to physically interact. Using surface force measurements, this has been determined to be $\sim 8 \mathrm{~nm}$ (Li, et al., 2007). To define the liposome area that contains all additional synaptobrevin-2 able to form a SNARE complex, we first consider the situation of a sphere of radius $r$ as it approaches a wall, so that its center is $r+d$ units of length from the wall (Figure 5.7). We wanted to know the surface area, $\mathrm{S}_{\mathrm{h}}$, of the sphere that is less than $h$ units away from the wall. We consider the surface area $\mathrm{S}$ of the entire sphere in polar coordinates: 

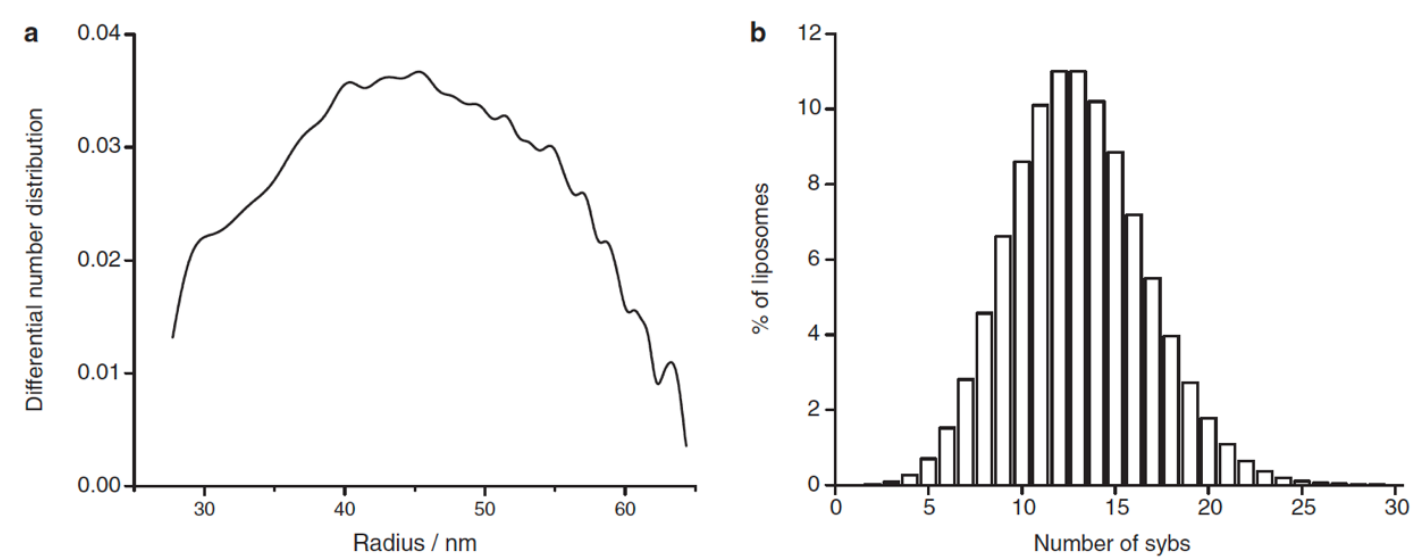

Figure 5.6: The population of large liposomes without any synaptobrevin-2 is negligible at the lipid mixing efficiency threshold and cannot explain the drop in lipid mixing on large liposomes. (A) Differential number distribution of a representative large synaptobrevin-2 liposome at lipid/protein = 400:1 obtained by FFF-MALLS. (B) To evaluate whether a population of large liposomes containing no synaptobrevin-2 could explain the lipid mixing decline on large liposomes, we assumed synaptobrevin-2 are distributed randomly and calculated a hypothetical Poisson distribution of the number of synaptobrevin-2 per liposome using the R-statistical software package (www.r-project.org). To be conservative, we used the corrected synaptobrevin-2/lipid ratio at the lower bound threshold (3.1 $\times 10^{-4}$, blue dashed line in Figure 5.5A) and assumed synaptobrevin-2 were reconstituted in $60 \mathrm{~nm}$-diameter liposomes, which is at the lower end of the size distribution in A. Assuming a bilayer thickness of $4 \mathbf{n m}$, the average number of synaptobrevin-2 per liposome is 13 (eq. 5.9). Even in this conservative scenario there are virtually no liposomes without synaptobrevin-2. Data was collected by Matias Hernandez. Figure is adapted from (Hernandez, et al., 2014). 


$$
S=4 \pi r^{2}=\iint_{0,0}^{2 \pi, \pi} r^{2} \sin \phi d \phi d \theta
$$

where $\theta$ belongs to the range $[0,2 \pi]$ and $\phi$ belongs to the range $[0, \pi]$. The surface area $\mathrm{S}_{\mathrm{h} \text {,wall }}$ is then given by

$$
S_{h, \text { wall }}=2 \pi r^{2} \int_{0}^{\phi_{h}} \sin \phi d \phi
$$

From the geometry shown in Figure 5.7, the angle $\phi_{\mathrm{h}}$ delimited by $h$ is given by

$$
\phi_{h}=\cos ^{-1} \frac{r-h+d}{r}
$$

Substituting and rearranging eqs. 5.11 and 5.12 givens

$$
S_{h, w a l l}=2 \pi r^{2}\left[\cos \left(\cos ^{-1} \frac{r-h+d}{r}\right)-1\right]=2 \pi r^{2} \frac{(h-d)}{r}=2 \pi r(h-d)
$$

To model the fusion between two liposomes, we used the geometry portrayed in Figure 5.7B to derive the expression for $\phi_{\mathrm{h}}$. In this case where the radii of two spheres of radius $r$ are separated by $2 r+d$ units, $\phi_{\mathrm{h}}$ is given by

$$
\phi_{h}=\cos ^{-1} \frac{r-\frac{h}{2}+\frac{d}{2}}{r}
$$

Substituting this expression into eq. 5.11 then gives the surface area $\mathrm{S}_{\mathrm{h} \text {,sphere: }}$ :

$$
S_{h, \text { sphere }}=2 \pi r^{2} \frac{(h-d)}{2 r}=\pi r(h-d)
$$

The number of additional synaptobrevin-2s confined within the area delimited by $h\left(\mathrm{syb}_{\mathrm{h}}\right)$ that are readily within reach for SNARE complex formation is then given by:

$$
s y b_{h}=\left(s y b_{\text {total }}-1\right) \times \frac{s_{h, \text { sphere }}}{s}=\left(s y b_{\text {total }}-1\right) \times \frac{(h-d)}{4 r}
$$

where syb $\mathrm{b}_{\text {total }}$ is the total number of synaptobrevin- 2 on a liposome as estimated according to eq. 5.9. Because in our model one synaptobrevin-2 is already engaged in SNARE complex assembly, which mediates the docking, the number of synaptobrevin-2 that would be required for efficient lipid mixing assuming a cooperative mechanism in $\mathrm{syb}_{\mathrm{h}}+1$. This leads to two to four 
synaptobrevin-2 (of the 23-30 synaptobrevin-2 present) would be instantly within reach to form SNARE complexes, Table 5.1. However, we cannot discard that more SNARE complexes may be recruited with or without expansion of the contact zone before fusion is experimentally observed.

Based on these considerations we suggest two models: (i) Fusion initiates at a single contact site and requires a localized and synchronous cooperative assembly of SNAREs requiring only a subset of the 23-30 synaptobrevin-2, Figure 5.8 A and B, or (ii) fusion involves and expanded contact site that forms over time as a larger number of cooperating SNAREs are recruited over larger distances, Figure 5.8 C and D. 


\begin{tabular}{|c|c|c|c|c|c|c|}
\hline Scenario & $\begin{array}{l}\text { Sybs per lipid at } \\
\text { efficiency threshold } \\
\text { (from Fig. 4A) }\end{array}$ & $\begin{array}{l}\text { Average } \\
\text { radius, } \mathrm{nm}\end{array}$ & $\begin{array}{c}\text { Bilayer } \\
\text { thickness, } \mathrm{nm}\end{array}$ & $\begin{array}{l}\text { No. of sybs } \\
\text { per liposome }\end{array}$ & $\begin{array}{c}\text { Maximum distance } \\
\text { SNARE interaction } \\
h, \mathrm{~nm}\end{array}$ & $\begin{array}{l}\text { No. of sybs at } \\
\text { fusion site } \\
\left(\text { syb }_{h}+1\right)\end{array}$ \\
\hline Lower boundary & $3.1 \times 10^{-4}$ & 40 & 4 & 18.2 & 5 & 1.5 \\
\hline Average & $3.6 \times 10^{-4}$ & 45 & 4 & 26.8 & 8 & 2.2 \\
\hline Upper boundary & $4.2 \times 10^{-4}$ & 50 & 5 & 47.7 & 12 & 3.8 \\
\hline
\end{tabular}

Table 5.1: Estimation of number of SNARE complexes that would readily form at the docking interface delimited by the maximum distance over which SNAREs can interact. We used eqs. 5.9 and 5.16 to evaluate how many synaptobrevin-2 would form trans SNARE complexes in a local and concerted fashion under three distinct scenarios. For lower- and upper-bound scenarios, we used the synaptobrevin-2/lipid lipid mixing thresholds from the lower and upper limits of the fitting (dashed lines in Figure 5.5A) and the average fitting for the average scenario (blue solid line in Figure 5.5A). In addition, we tested different model parameters appropriate for each scenario that include the radius $r$, the bilayer thickness $b$, and the maximum distance of SNARE interaction $h$ to evaluate the variability of the output of our model. Based on this analysis, we estimate that between two and four synaptobrevin-2 would form SNARE complexes assuming a localized and concerted cooperative mechanism. Therefore, between two and four SNARE complexes would be required for efficient lipid mixing. Calculations were performed by Matias Hernandez. Table is adapted from (Hernandez, et al., 2014). 

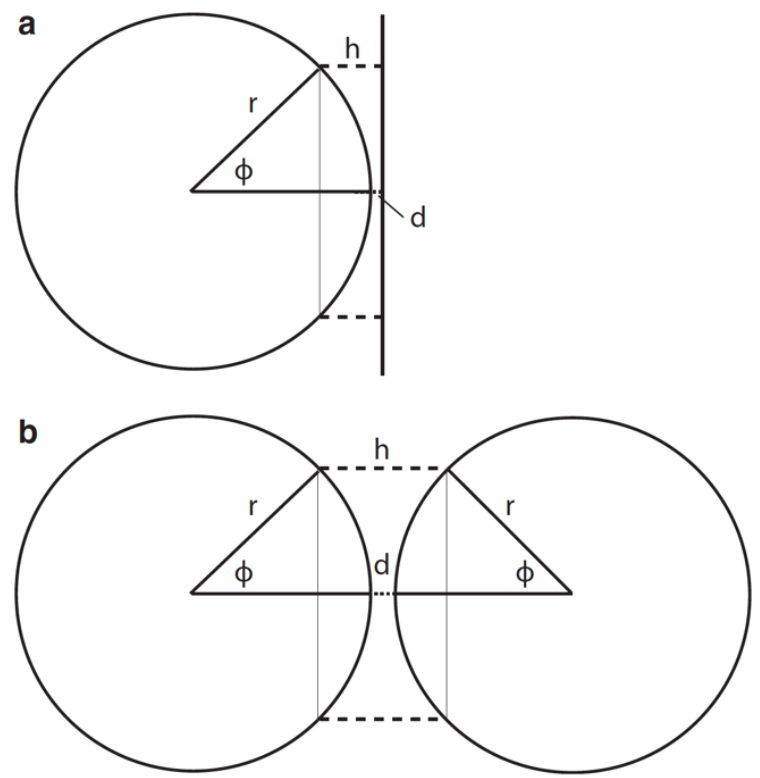

Figure 5.7: Geometric model for calculating the surface area of the syb2 liposomes that is deliminated by the maximum distance of the membranes by which SNARE partners can interact. (A) Geometry between a sphere and a wall and (B) between two spheres of equal radii $r$ whose surfaces are separated by a distance $d$. We designed the liposome fusion assay so that the $\Delta N$ complex is present in excess and covers a large portion of the surface. The estimated number of SNARE complexes that can potentially form in this model is based on the assumption that, as the distance between the membranes $d$ approaches zero, all synaptorevin-2 in the area confined by the parameters $h, r$, and $\phi$ will initiate SNARE complex assembly. However, the model is limited by dynamic and steric effects of the SNAREs themselves (which would decrease this estimate) or lateral diffusion (which would increase the estimated number), and as such should be treated as only a rough approximation. Geometric model was designed by Matias Hernandez. Figure is adapted from (Hernandez, et al., 2014). 


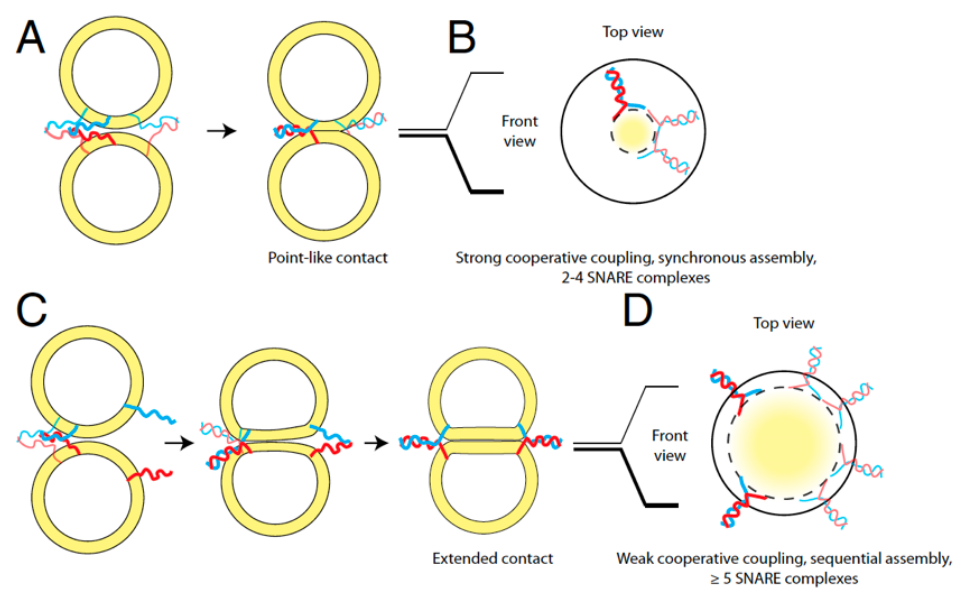

Figure 5.8: Cooperativity in SNARE-mediated fusion of large liposomes may be rationalized with two distinct coupling modes and may be further related to the morphology of the prefusion intermediated. (A) Strong cooperativity coupling. If a sufficient number of SNAREs are locally present at the moment of assembly of the first trans SNARE complex, additional SNARE complexes assemble in a near-synchronous manner. We refer to this scenario as strong coupling between SNARE complexes owing to the exerted force being focused on a point-like contact zone, which initiates membrane fusion. SNARE complexes drawn with bolder/fainter lines denote those oriented toward/away from the observer to give a 3D impression of the spatial arrangement. (B) Projection through membrane contact zone of A depicting the localized organization of the SNARE complexes in a strongly coupled mode. Based on the lipid mixing efficiency threshold on large liposomes and a simple geometric model, we estimate two to four SNARE complexes could assemble in this mode. This scenario results in the formation of local deformations or point-like protrusions of the membranes, as supported by very recent sparsely distributed in the membrane, the assembly of the first trans SNARE complex brings two extended bilayers together but does not immediately fuse them because it does not provide sufficient energy for surpassing the energy barrier. Additional SNAREs may be recruited from further away to assemble into complexes and exert force between the membranes over an extended contact area. We refer to this scenario as the weakly coupled cooperative mode. This weak coupling produces an extended contact zone between opposing membranes, a state that has been observed by cryo-electron microscopy of large liposomes (Hernandez, et al., 2012; Diao, et al., 2012). (D) Projection through the membrane contact zone of C depicting the spatial organization of the SNARE complexes in the weakly coupled cooperativity. The dashed circle denotes the projected borders of the contact area between the membranes, and the solid circle is the outer diameter of the liposomes. Model illustration was drawn by Matias Hernandez. Figure is adapted from (Hernandez, et al., 2014). 


\subsection{Discussion}

The systematic comparison of the fusion of small and large SNARE liposomes presented here leads us to propose mechanistic features of SNARE-mediated fusion that deserve further attention. We have uncovered a high variability in cooperativity in a controlled in vitro system where the curvature of the membranes is decreased only two- to threefold, leading us to presuppose that in vivo even greater variability is to be expected. Our results suggest that when the energy barrier is low, as in highly curved small liposomes, as few as one SNARE complex can suffice. However, fusion between large liposomes or in fusion of small liposomes with a planar membrane more SNAREs are needed to work in a cooperative manner to overcome the higher barrier. Thus, there seems to be no mechanistic requirement for a specific supramolecular rearrangement of SNAREs at the site of fusion. Because we observe this high variability with neuronal SNAREs at constant density but different curvature, a corollary of this conclusion is that we would expect that cooperativity of SNAREs involved in other trafficking pathways will also be highly variable, not because of their inherent biochemical properties, but rather owing to the particular biophysical characteristics of the trafficking vesicle. We therefore suggest that the reported estimates of numbers of involved SNARE complexes likely reflect the particular type of fusion studied and that there is no universally conserved number, as has been often assumed (van den Bogaart, et al., 2010; Sinha, et al., 2011; Shi, et al., 2012; Karatekin, et al., 2010; Domanska, et al., 2009; Hua \& Scheller, 2001).

Our results highlight the importance of correcting for docking rates to uncover cooperativity in bulk liposome fusion experiments. In a recent study, (Shi, et al., 2012), used a lipid mixing and a 
content release assay to investigate cooperativity in SNARE-mediated liposome-to-Nano disk fusion. As the authors pointed out, the rate-limiting step in these systems is docking due to slow initiation of SNARE complex assembly. (Shi, et al., 2012) found their lipid mixing unchanged (in both efficiency and speed) event after a ninefold change in synaptorevin-2 density, which is surprising because such behavior is incompatible with a docking rate-limited step. Content release, however, a roughly decreased as synaptobrevin-2 density was reduced, a finding that was attributed to a requirement for several synaptobrevin-2 to keep the fusion pore open. Although the observation that the lipid mixing dependence on SNARE density was unaltered remains to be explained, a simpler explanation for the SNARE density-dependent content release is that this behavior was due to slower docking rates at the lower SNARE densities.

Our observation that the efficiency threshold of 23-30 synaptobrevin-2 for the fusion of large liposomes leaves open questions about how cooperativity operates. What is, then, the mechanistic interpretation of this high number? The efficiency threshold definition we have used here refers to the total number of synaptobrevin-2 on a liposome at the point at which we observe a decrease in fusion (after taking docking into account). Our assumption has been that synaptobrevin-2 adjacent to the first trans SNARE complex would readily form additional SNARE complexes (because $\Delta \mathrm{N}$ complex is present in excess). However, at 23-30 synaptobrevin-2 this assumption is not straightforward to apply owing to steric factors. Furthermore, estimates of the number of SNAREs involved in the homotypic fusion of early endosomes and the exocytosis of chromaffin granules are much lower than the total number of 
SNAREs available, suggestion that only local subsets of SNAREs take part in fusion (Mohrmann, et al., 2010; Bethani, et al., 2009).

Based on these considerations, we have proposed two alternative mechanistic interpretations to explain our observations. In the first model, we suggest that although the threshold occurs at 2330 synaptobrevin- 2 on the entire surface of the liposome this is the total amount required to locally enrich SNARE complexes, a process that implies stronger coupling and time synchronization in the cooperative assembly of the SNARE complexes. We have modeled a possible scenario and found that two to four SNARE complexes assembled in synchrony would fit in between two large liposomes as contact between membranes is initiated, in agreement with in vivo studies (Mohrmann, et al., 2010; Sinha, et al., 2011; Hua \& Scheller, 2001). However, we caution that this is a lower bound estimate because we cannot rule out lateral diffusion of strong cooperative coupling between SNARE complexes implies that membrane fusion is confined to small contact areas leading to protrusions (Figure 5.5 A and B), a feature that has been recently identified by electron microscopy (Bharat, et al., 2014).

The second model we have considered involves SNARE complexes assembling at not one but multiple sites, which entails a weaker mode of cooperativity that operates over longer distances, Figure 5.8 C and D. Recent electron microscopy studies have depicted contact zones as large as $90 \mathrm{~nm}$ in diameter, which could accommodate the assembly of greater numbers of SNARE complexes (Hernandez, et al., 2012; Diao, et al., 2012). Interestingly, the morphology of this 
putative intermediate resembles very closely the vertex ring observed on yeast vacuoles, begging the question as to whether a feature of weak cooperative coupling is to generate extended docking intermediates such as those found in slower constitutive forms of fusion (Wang, et al., 2002). In this regard, cooperativity may also critically depend on the required speed of a particular fusion reaction, an idea that we have not explored in the current work but that has been conceived in the context of dense core vesicle fusion in chromaffin cells (Mohrmann, et al., 2010). It is plausible that, for example, synaptic vesicle fusion, which requires fast speed, might proceed via a mechanism requiring much stronger coupling and thus higher cooperativity between SNAREs than is observed for the slower vacuolar fusion.

We recognize possible limitations arising from the use of the $\Delta \mathrm{N}$ complex, which we used here to quantitatively fix the 1:1 syx:SNAP25 acceptor complex. In particular, the displacement of the syb49-96 peptide requires overcoming a high activation energy barrier that reduces the force transmitted to the membranes (Hernandez, et al., 2012). Although this does not alter our conclusion that cooperativity is variable, it does imply that the cooperativity threshold between large liposomes might be overestimated. Another factor to consider is that displacement of the fragment may be rate-limiting and thereby hide cooperativity with regards to the speed of lipid mixing. Thus, the cooperativity we have uncovered specifically refers to the efficiency of lipid mixing and it is understood that we cannot rule out another level of cooperativity with respect to speed. 
Despite these limitations, we also note that use of the $\Delta \mathrm{N}$ complex in our study may have unraveled an otherwise occluded cooperativity behavior. When using full-length SNAREs, only a very low percentage of large SNARE liposomes undergo fusion and instead remain either stably docked or hemi-fused even at very high lipid/protein ratios of 50 and 200:1 (Kyoung, et al., 2011; Lai, et al., 2013)., a finding we have also observed in our bulk assays. It is not clear why this is the case, but the observation that synaptotagmin- 1 and $\mathrm{Ca}^{2+}$ substantially enhances fusion by presumably lowering the activation energy of certain intermediates might suggest that the energy barriers in these systems are higher than in ours. However, an alternative explanation may be that synchronized cooperative assembly of SNAREs is jeopardized by the dynamic generation of off-pathway 2:1 syx:SN25 acceptor complexes. This would reduce the effective concentration of 1:1 acceptor complexes at the site of fusion and desynchronize the strong cooperative coupling between complexes required for the execution of fusion. 


\section{Chapter 6: High cholesterol obviates a prolonged}

\section{hemifusion intermediate in SNARE-mediated}

\section{membrane fusion}

Kreutzberger, A.J.B., V. Kiessling, and L.K. Tamm (2015) Biophysical Journal, 109:319-329.

Yang, S.T., A.J.B. Kreutzberger, J. Lee, V. Kiessling, L.K. Tamm (2016) Chem Phys Lipids, 199:136-143.

\subsection{Summary}

Cholesterol is essential for exocytosis in secretory cells, but the exact molecular mechanism by which it facilitates exocytosis is largely unknown. Distinguishing contributions from the lateral organization and dynamics of membrane proteins to vesicle docking and fusion and the promotion of fusion pores by negative intrinsic spontaneous curvature and other mechanical effects of cholesterol have been elusive. To shed more light on this process, we examined the effect of cholesterol on SNARE-mediated membrane fusion in a single-vesicle assay that is capable of resolving docking and elementary steps of fusion with millisecond time resolution. The effect of cholesterol on fusion pore formation between synaptobrevin-2 (VAMP-2)containing proteoliposomes and acceptor t-SNARE complex-containing planar supported bilayers was examined using both membrane and content fluorescent markers. This approach revealed that increasing cholesterol in either the t-SNARE or the v-SNARE membrane favors a mechanism of direct fusion pore opening, whereas low cholesterol favors a mechanism leading to a long-lived ( $>5 \mathrm{~s}$ ) hemifusion state. The amount of cholesterol in the target membrane had no 
significant effect on docking of syb2 vesicles. Comparative studies with $\alpha$-tocopherol (vitamin E) show that the negative intrinsic spontaneous curvature of cholesterol and its presumed promotion of a very short-lived $(<50 \mathrm{~ms})$ lipid stalk intermediate is the main factor that favors rapid fusion pore opening at high cholesterol. This study also shows that this single-vesicle fusion assay can distinguish between hemifusion and full fusion with only a single lipid dye, thereby freeing up a fluorescence channel for the simultaneous measurement of another parameter in fast time-resolved fusion assays.

\subsection{Introduction}

Membrane cholesterol is well known to be necessary for efficient exocytosis of synaptic vesicles in neurons (Linetti et al., 2010; Wasser, Ertunc, Liu, \& Kavalali, 2007; Zamir \& Charlton, 2006), endocrine cells (Hao \& Bogan, 2009), neuroendocrine cells (Koseoglu, et al., 2011; Zhang, et al., 2009), and cortical vesicles in sea urchins (Churchward, et al., 2005; Churchward, et al., 2008;

Churchward \& Coorssen, 2009). Cholesterol has also been shown to recruit SNAREs and other secretory proteins into clusters in the plasma membranes of secretory cells (Chamberlain, et al., 2001; Gil, et al., 2005; Lang, et al., 2001; Lang, 2007; Salaün, et al., 2005; Salaün, et al., 2005; Taverna, et al., 2004; Taverna, et al., 2007; van den Bogaart, et al., 2013). Although clearly dependent on cholesterol, these clustered protein domains differ from classical lipid rafts because they are not co-labeled with typical raft markers (Lang, 2007). Cholesterol-dependent clustering of synatixin-1a have also been found when syntaxin-1a was reconstituted into single-phase model membranes that were devoid of any lipid rafts (Murray \& Tamm, 2009; Murray \& Tamm, 2011). In addition, cholesterol is highly enriched in secretory vesicles (Deutsch \& Kelly, 1981; 
Thiele, et al., 2000; Takamori, et al., 2006) where is has been implicated in promoting membrane fusion (Churchward, et al., 2005; Churchward, et al., 2008). Previous reconstitution studies have shown that cholesterol accelerates SNARE mediated fusion in lipid mixing assays (Chang, et al., 2009; Tong, et al., 2009), but the reasons for this acceleration were only partially addressed.

Although it is clear that cholesterol plays an important role in SNARE-mediated membrane fusion, the molecular mechanism by which it facilitates fusion remains elusive. Possible explanations for cholesterol's role in fusion might include the segregation of membrane proteins into domains (Lang, et al., 2001; Taverna, et al., 2007; Taverna, et al., 2004; Salaün, et al., 2005; Salaün, et al., 2005; Gil, et al., 2005; Lang, 2007; van den Bogaart, et al., 2013; Lingwood \& Simons, 2010; Simons \& Gerl, n.d.) its ability to order membrane lipids (Yeagle, 1985; Sankaram \& Thompson, 1990) and changing membrane fluidity (Recktenwald \& McConnell, 1981), as well as its potential to stabilize negatively curved lipid structures that are thought to be intermediates on the path to membrane fusion (Coorssen \& Rand, 1990; Chen \& Rand, 1997; Wang, et al., 2007). A complete summary of the effects of cholesterol of membranes is illustrated in Figure 6.1. These different modes of action of cholesterol could potentially apply to either increase the rates of docking of $\mathrm{v}$ - and t-SNARE vesicles or they could increase the intrinsic rates of fusion after cognate SNARE vesicles have docked. 
a

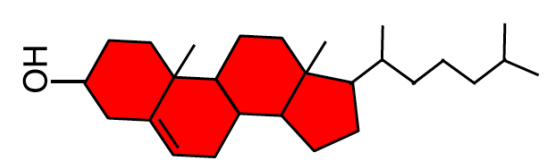

b

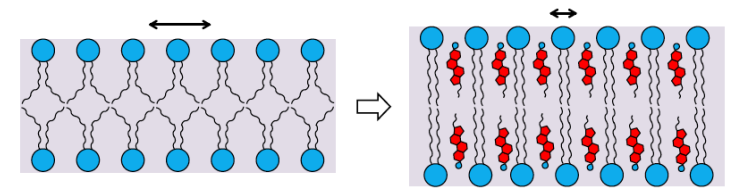

C

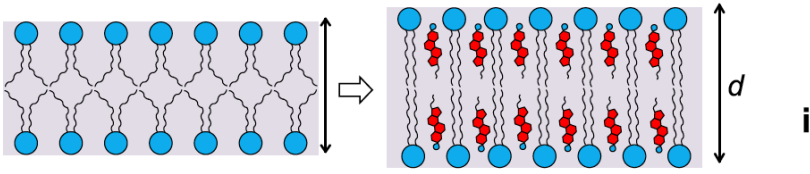

d

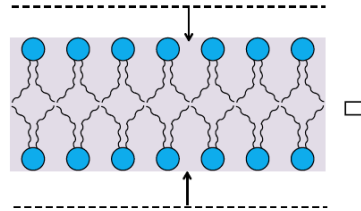

e
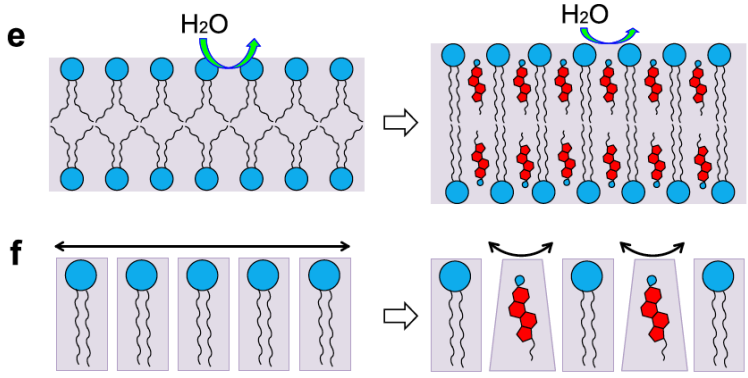

g

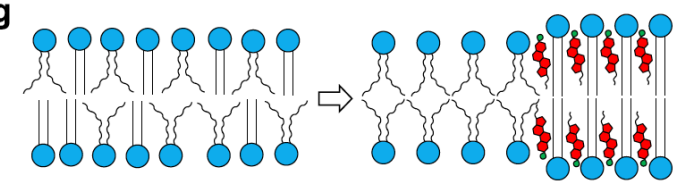

h

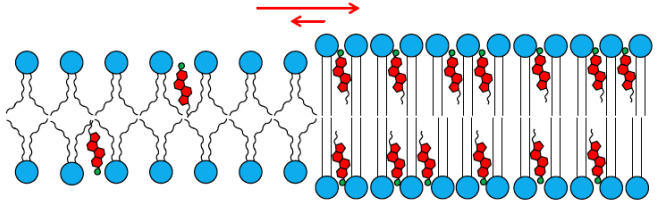

i

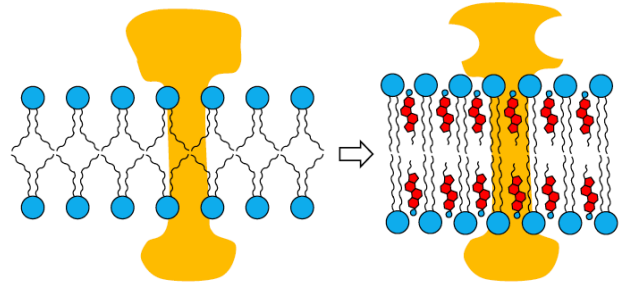

j

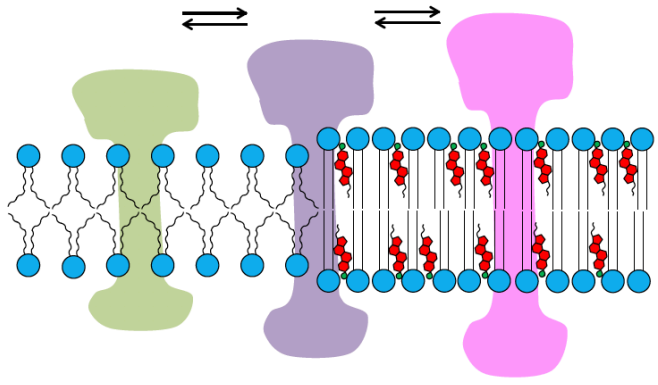

Figure 6.1: Cholesterol has multiple effects on lipid bilayers. Cholesterol (A) changes the fluidity (B), thickness (C), compressibility (D), water penetration (E), and intrinsic curvature (F) of lipid bilayers. Cholesterol also induces phase separations in multicomponent lipid mixtures (G), partitions selectively between different coexisting lipid phases $(\mathrm{H})$, and causes integral membrane proteins to respond by changing conformation (I) or redistribution $(J)$ in the membrane. This cartoon was drawn by Sung-Tae Yang. This figure is adapted from (Yang, et al., 2016). 


\subsection{Results}

Four characteristic events of fusion were distinguished by simultaneously imaging a membrane being (I) docking (without fusion), (II) hemi-fusion, (III) direct full fusion, and (IV) two-step fusions with a resolvable hemifusion intermediate. These characteristic fusion events are shown in Figure 3.3 and discussed in detail in Chapter 3. The fusion of syb2 proteoliposomes with a constant $20 \mathrm{~mol} \%$ concentration of cholesterol was monitored with increasing concentrations of cholesterol in the planar supported target membrane, Figure 6.2. Changes in target membrane cholesterol did not affect overall protein concentrations or their efficiencies of insertion into the proteoliposomes that were used to form the planar supported bilayers, Figure 6.3. In the absence of cholesterol, $\sim 80 \%$ of all fusion events were hemi-fusion events. However, when cholesterol was increased in the target membrane, direct full fusion events increased gradually until they reached $\sim 80 \%$ of all fusion events at $40 \mathrm{~mol} \%$ cholesterol (Figure 6.2A). We extracted the delay times between docking and the onset of fusion or hemi-fusion for all events. Normalized cumulative distribution functions of these time delays were constructed as shown in Figure 2B and $\mathbf{C}$, respectively. These are kinetic curves representing the fusion and hemi-fusion reactions, respectively, without any contribution from vesicle docking. The kinetic data were fit to several models including the following very simple first order rate law:

$$
\mathrm{A} \rightarrow \mathrm{B}
$$

and the following rate law with two consecutive steps:

$$
\mathrm{A} \rightarrow \mathrm{B} \rightarrow \mathrm{C}
$$

characterized by two different rate constants, $\mathrm{k}_{1}$ and $\mathrm{k}_{2}$. The single-step rate law did not result in good fits for all experimental data, whereas the two-step rate law reproduced the experimental 
data very well. More complex kinetic models were not needed to explain the data as was the case for the fast component of SNARE mediated fusion using synthetic lipids (Domanska, et al., 2009; Domanska, et al., 2010). A summary of all recorded events under different conditions is shown in Table 6.1. The rate constants derived from the kinetic fits of the two-consecutive step model are shown in Table 6.2. The processes are characterized by a slower rate of $\sim 1 \mathrm{~s}^{-1}$ that is quite independent of the type of fusion and the cholesterol concentration and a faster rate on the order of 3 to $20 \mathrm{~s}^{-1}$ that is a little more, but not strongly process- and cholesterol-dependent. The kinetic models do not a priori assign the order of the fast and slow processes in the above scheme. While cholesterol greatly altered single vesicle fusion efficiencies and delay kinetics between docking and fusion no effect on cholesterol and syb2 proteoliposome docking was observed, Figure 6.3. The data shown in Figure 6.2 was used to determine if the membrane dye was sufficient to distinguish hemi- and full- fusion, Figure 3.5, and is discussed in Chapter 3. 


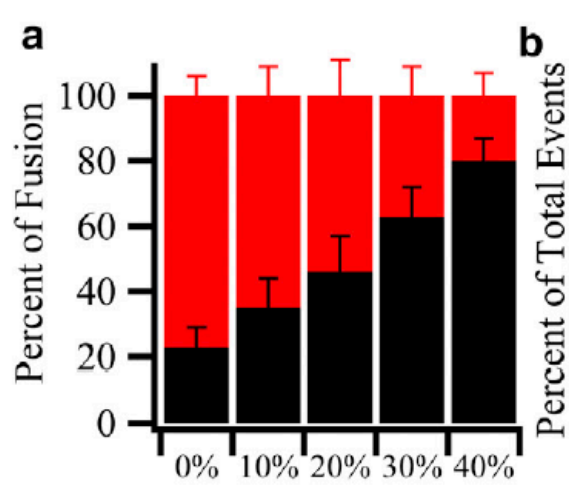

Cholesterol

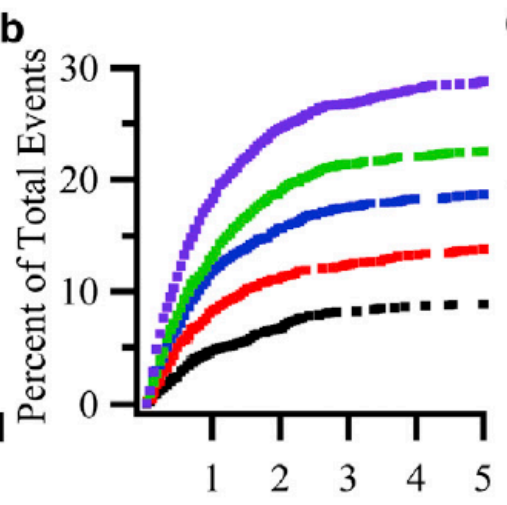

Time (s)

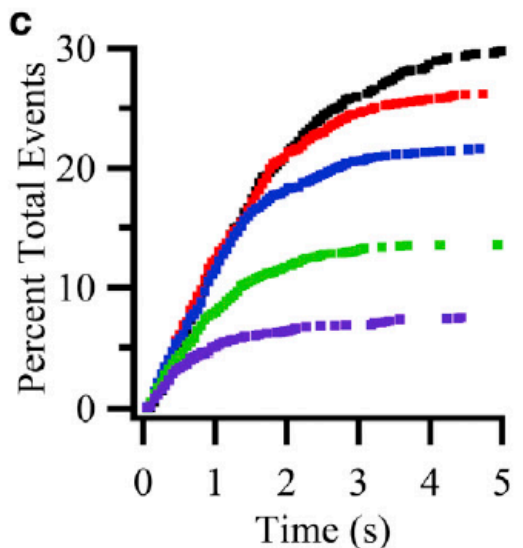

Time (s)

Figure 6.2: Summary of direct full fusion and hemi-fusion events obtained from single0vesicle fusion assays as a function of cholesterol in the planar supported target membrane. (a) Relative fractions of direct full fusion (black) and hemi-fusion (red) at different mol \% of cholesterol in the planar target membrane after 5 s. The numerical values are $23 \pm 6 \%, 35 \pm 9 \%, 46 \pm 11 \%, 63 \pm 9 \%$, and $80 \pm 7 \%$ full fusion and $77 \pm 6 \%, 65 \pm 9 \%, 54 \pm 11 \%, 37 \pm 9 \%$, and $20 \pm 7 \%$ hemi-fusion of the total number of fusion events (total numbers are shown in Table 6.1), respectively, at $0,10,20,30$, and $40 \mathrm{~mol} \%$ cholesterol. (b) Cumulative distribution functions of direct full fusion events normalized to the efficiencies of total docking events, which were $9 \pm 3,14 \pm 4 \%, 19 \pm 6 \%, 22 \pm 5 \%$, and $29 \pm 5 \%$ for (from bottom to top) 0 (black), 10 (red), 20 (blue), 30 (green), and 40 (purple) mol $\%$ cholesterol, respectively, in the planar target membrane. (c) Cumulative distribution function of hemi-fusion events normalized to the efficiencies of total docking events, which were $30 \pm 6 \%, 26 \pm$ $6 \%, 22 \pm 6 \%, 14 \pm 5 \%$, and $7 \pm 4 \%$ for (from top to bottom) 0 (black), 10 (red), 20 (blue), 30 (green), and 40 (purple) mol \% cholesterol, respectively, in the planar target membrane. This figure was adapted from (Kreutzberger, et al., 2015). 


\begin{tabular}{lcccc}
\hline $\begin{array}{l}\text { Cholesterol } \\
(\%)\end{array}$ & $\begin{array}{c}\text { Total No. of } \\
\text { Direct Full } \\
\text { Fusion Events }\end{array}$ & $\begin{array}{c}\text { Total No. of } \\
\text { Hemifusion } \\
\text { Events }\end{array}$ & $\begin{array}{c}\text { Total No. of } \\
\text { Docked } \\
\text { Liposomes }\end{array}$ & $\begin{array}{c}\text { No. of } \\
\text { Experiments }\end{array}$ \\
\hline 0 & 132 & 437 & 1418 & 8 \\
10 & 253 & 472 & 1855 & 12 \\
20 & 370 & 412 & 1973 & 7 \\
30 & 537 & 313 & 2520 & 8 \\
40 & 604 & 142 & 2105 & 7 \\
\hline
\end{tabular}

Table 6.1: Summary of events at different cholesterol concentrations in the planar supported bilayer. These data were used to generate Figure 6.2A and to normalize Figure 6.2B and C. The error bars in Figure $2 \mathrm{~A}$ represent the averages of all experiments under each condition. This data is adapted from (Kreutzberger, et al., 2015).

\begin{tabular}{lccccc}
\hline & \multicolumn{2}{c}{ Direct Full Fusion } & & \multicolumn{2}{c}{ Hemifusion } \\
\cline { 2 - 3 } \cline { 5 - 6 } Cholesterol $(\%)$ & $\mathrm{k}_{1}\left(\mathrm{~s}^{-1}\right)$ & $\mathrm{k}_{2}\left(\mathrm{~s}^{-1}\right)$ & & $\mathrm{k}_{1}\left(\mathrm{~s}^{-1}\right)$ & $\mathrm{k}_{2}\left(\mathrm{~s}^{-1}\right)$ \\
\hline 0 & $0.73 \pm 0.06$ & $18 \pm 7$ & & $0.75 \pm 0.06$ & $2.9 \pm 0.4$ \\
10 & $1.00 \pm 0.06$ & $16 \pm 4$ & & $0.80 \pm 0.05$ & $4.6 \pm 0.6$ \\
20 & $1.00 \pm 0.04$ & $22 \pm 5$ & & $0.91 \pm 0.05$ & $6.3 \pm 0.9$ \\
30 & $0.96 \pm 0.04$ & $15 \pm 2$ & & $0.97 \pm 0.06$ & $10 \pm 2$ \\
40 & $1.17 \pm 0.04$ & $13 \pm 2$ & & $1.4 \pm 0.1$ & $11 \pm 3$ \\
\hline
\end{tabular}

Table 6.2: Rate constants $\left.\left(\mathrm{k}^{-1} \mathrm{~s}^{-1}\right)\right)$ derived from fits of the data of Figure $2 \mathrm{~B}$ and $\mathrm{C}$ to the two-step model of direct full fusion and hemi-fusion. This data is adapted from (Kreutzberger, et al., 2015). 


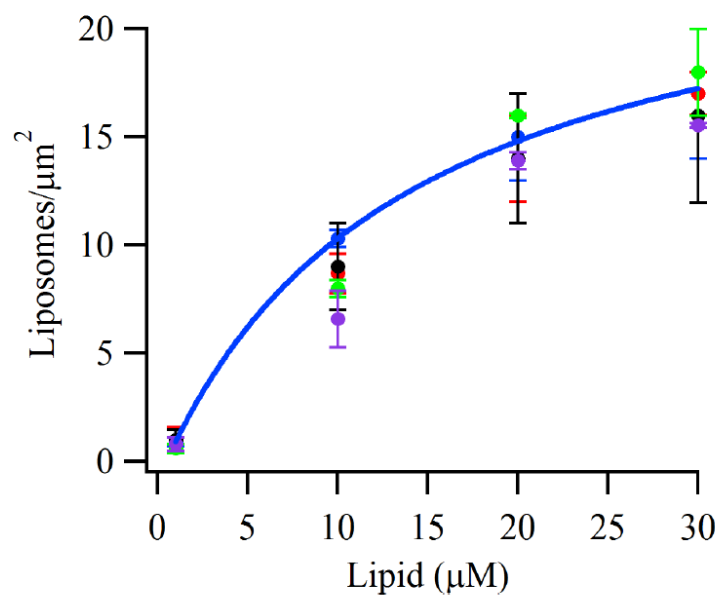

Figure 6.3: Docking of syb2 proteoliposomes on acceptor t-SNARE containing planar supported bilayers with different concentrations of cholesterol: 0 mol \% (black), 10 mol \% (red), 20 mol \% (blue), $30 \mathrm{~mol} \%$ (green), and $40 \mathrm{~mol} \%$ (purple). Error bars are standard deviations from 3 to 4 experiments. The data are plotted as number of synaptobrevin-2 liposomes bound per $\mu \mathrm{m}^{2}$ versus added syb2 proteoliposome lipid concentration. The blue curve shows the best fit of a Langmuir isotherm to $20 \%$ cholesterol data. This figure was adapted from (Kreutzberger, et al., 2015). 
a)

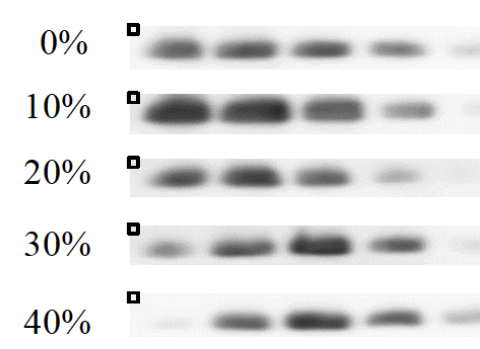

b)

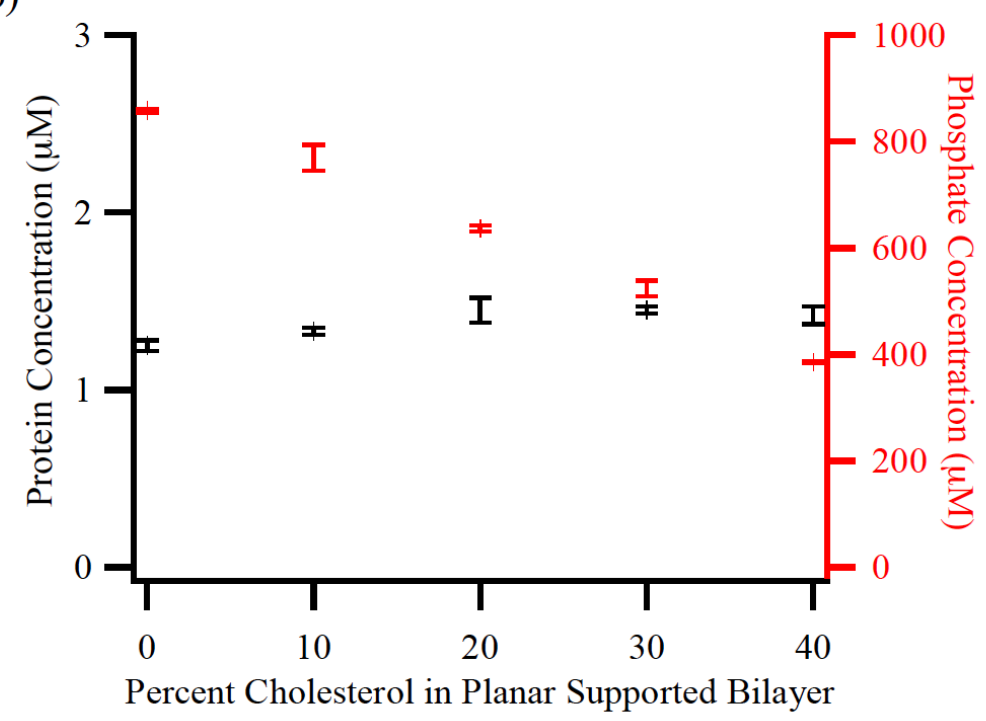

Figure 6.4: (A) Western blots of SNAP-25 from a co-floatation assay indicating full insertion of the acceptor t-SNARE complex for all cholesterol compositions used in the planar target membranes. Lanes from left to right indicated fractions from the top to the bottom of the leaflets of the planar supported target membranes as determined by the BCA and modified Bartlett assays, respectively. The results are from four independent reconstitutions of acceptor t-SNARE complex proteoliposomes. This figure was adapted from (Kreutzberger, et al., 2015). 
To determine if cholesterol in the vesicle membrane has similar effects on hemi- and full fusion as in the planar target membrane, we changed the cholesterol concentration in syb2 proteoliposomes from 0 to $40 \mathrm{~mol} \%$ while keeping the cholesterol concentration in the planar target membrane constant at $20 \mathrm{~mol} \%$. Because membrane curvature can influence the efficiency and kinetics of SNARE-mediated membrane fusion (Hernandez, et al., 2014), we first examined if cholesterol changed the size of the reconstituted syb2 vesicles. Indeed, when the cholesterol concentration was increased, the mean diameter of the proteoliposomes as determined by cryoelectron microscopy increased from $\sim 25 \mathrm{~nm}$ in the absence of cholesterol to $\sim 40 \mathrm{~nm}$ at 20 and $40 \mathrm{~mol} \%$ cholesterol (Figure 6.5). More examples of cryoelectron microscopy images are shown in Figure 6.6. The influence of cholesterol in the syb2 proteoliposomes on the fusion efficiencies was similar to the ones observed when cholesterol was increased in the acceptor complex-containing target membrane. The proportion of direct full fusion events increased substantially at the expense of long-lived hemi-fusion events when cholesterol was increased in the syb2 proteoliposomes (Figure 6.5D). The normalized cumulative distribution functions of the fusion delay times for direct full fusion and hemifusion are shown in Figure 6.5E and $F$, respectively. A summary of all recorded events under different conditions is shown in Table 6.3. The kinetic data could again be best fitted to the consecutive-step model and the derived kinetic rate constants are shown in Table 6.4. As with cholesterol in the planar target membrane, the two rates were on the orders of $1 \mathrm{~s}^{-1}$ and $10 \mathrm{~s}^{-1}$. 

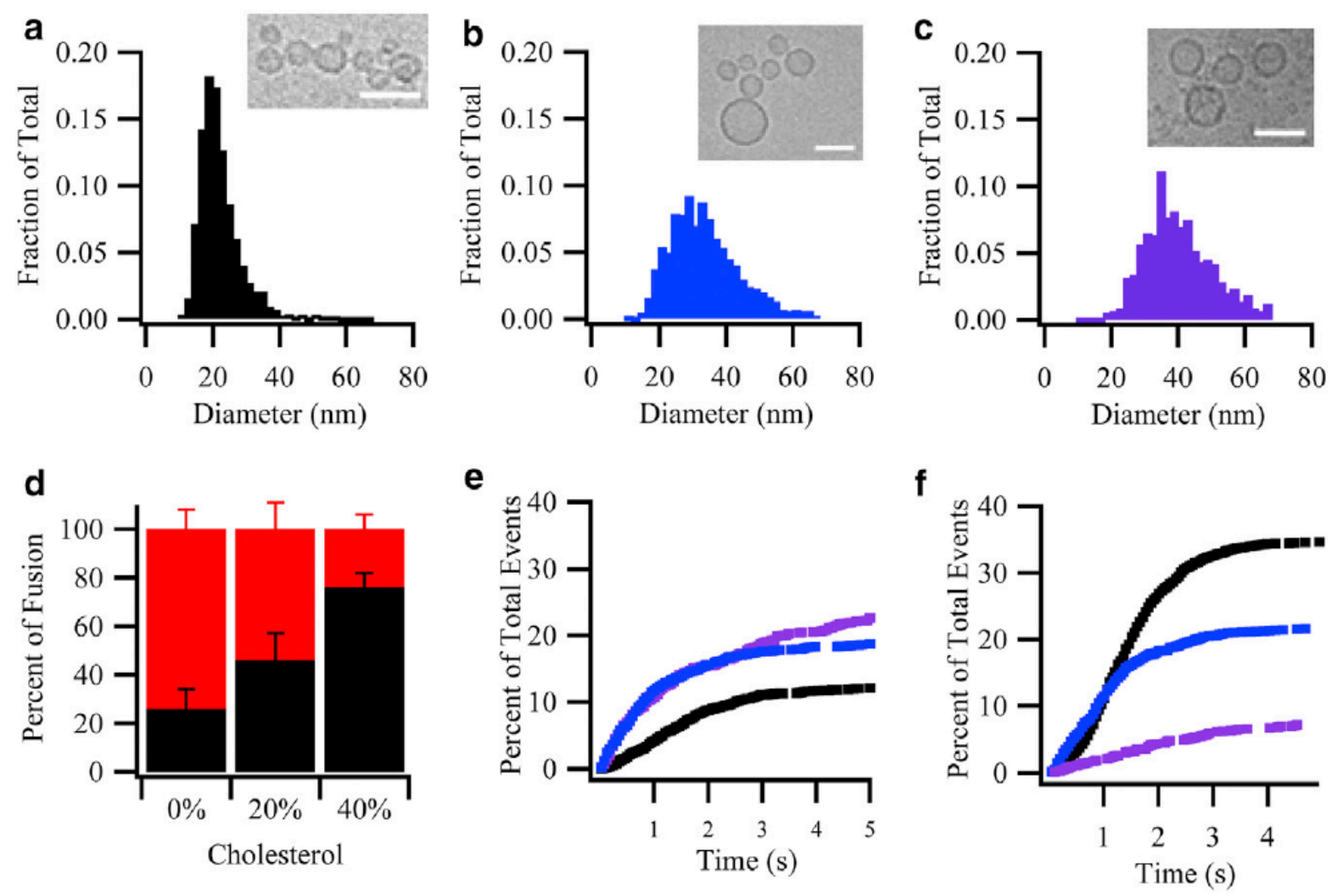

Figure 6.5: Summary of direct full fusio0n and hemifusion obtained from single-vesicle fusion assays as a function of cholesterol in syb2 proteoliposome membrane. (A-C) Size distributions of syb2 proteoliposomes containing (A) 0, (B) 20, and (C) $40 \mathrm{~mol} \%$ cholesterol. Insets are example images for each condition with scale bars of $50 \mathrm{~nm}$. (D) relative fractions of direct full fusion (black) and hemifusion (red) at different mol \% cholesterol in the syb2 proteoliposome membrane after $5 \mathrm{~s}$. The numerical values are $26 \pm 8 \%, 46 \pm 11 \%$, and $76 \pm 6 \%$ of direct full fusion and $74 \pm 8 \%, 54 \pm$ $11 \%$, and $24 \pm 6 \%$ hemi-fusion of the total number of fusion events (total numbers showing in Table 6.3), respectively, at 0,20 , and $40 \mathrm{~mol} \%$ cholesterol. (E) Cumulative distribution function of direct full fusion events normalized to the efficiencies of total docking events, which were $12 \pm 4 \%$, $19 \pm 6 \%$, and $23 \pm 2 \%$ of total number of events for 0 (black), 20 (blue), and 40 (purple) mol \% cholesterol, respectively, in the syb2 proteoliposome membrane. (F) Cumulative distribution function of hemifusion events normalized to the efficiencies of total docking events, which were $35 \pm$ $7 \%, 22 \pm 6 \%$, and $7 \pm 2 \%$ hemi-fusion of the total number of events for 0 (black), 20 (blue), and 40 (purple) mol \% cholesterol, respectively, in the syb2 proteoliposome membrane. This figure was adapted from (Kreutzberger, et al., 2015). 


\begin{tabular}{lcccc}
\hline $\begin{array}{l}\text { Cholesterol } \\
(\%)\end{array}$ & $\begin{array}{c}\text { Total No. of } \\
\text { Direct Full } \\
\text { Fusion Events }\end{array}$ & $\begin{array}{c}\text { Total No. of } \\
\text { Hemifusion } \\
\text { Events }\end{array}$ & $\begin{array}{c}\text { Total No. of } \\
\text { Docked } \\
\text { Liposomes }\end{array}$ & $\begin{array}{c}\text { No. of } \\
\text { Experiments }\end{array}$ \\
\hline 0 & 185 & 418 & 1524 & 5 \\
20 & 400 & 412 & 1973 & 7 \\
40 & 243 & 78 & 1073 & 5 \\
\hline
\end{tabular}

Table 6.3: Summary of events at different cholesterol concentrations in the syb2 proteoliposomes. These data were used to generate Figure 6.5D and to normalize Figure 6.5 E and F. There error bars in Figure $4 \mathrm{D}$ represent the averages of all experiments under each condition. This data was adapted from (Kreutzberger, et al., 2015).

\begin{tabular}{lcclcc}
\hline & \multicolumn{2}{c}{ Direct Full Fusion } & & \multicolumn{2}{c}{ Hemifusion } \\
\cline { 2 - 3 } \cline { 5 - 6 } Cholesterol $(\%)$ & $\mathrm{k}_{1}\left(\mathrm{~s}^{-1}\right)$ & $\mathrm{k}_{2}\left(\mathrm{~s}^{-1}\right)$ & & $\mathrm{k}_{1}\left(\mathrm{~s}^{-1}\right)$ & $\mathrm{k}_{2}\left(\mathrm{~s}^{-1}\right)$ \\
\hline 0 & $0.9 \pm 0.2$ & $1.7 \pm 0.4$ & & $1.16 \pm 0.03$ & - \\
20 & $1.00 \pm 0.04$ & $22 \pm 5$ & & $0.91 \pm 0.05$ & $6.3 \pm 0.9$ \\
40 & $0.70 \pm 0.04$ & $33 \pm 17$ & & $0.31 \pm 0.07$ & $8 \pm 4$ \\
\hline
\end{tabular}

Table 6.4: Rate constants $\left(\mathrm{k}^{\left.\left(\mathrm{s}^{-1}\right)\right)}\right.$ derived from fits of the data of Figure 6.5E and $\mathrm{F}$ to the two-step model of direct full fusion and hemi-fusion. This data was adapted from (Kreutzberger, et al., 2015). 
a)

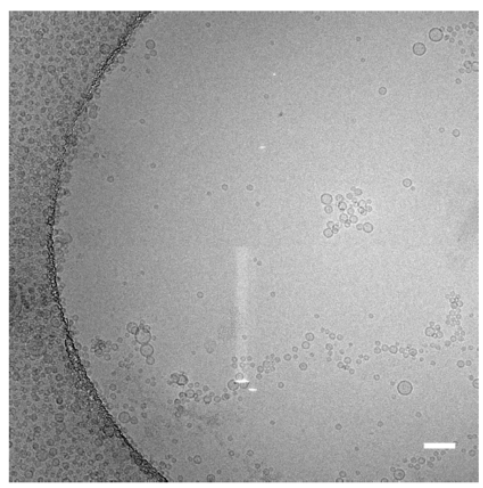

b)

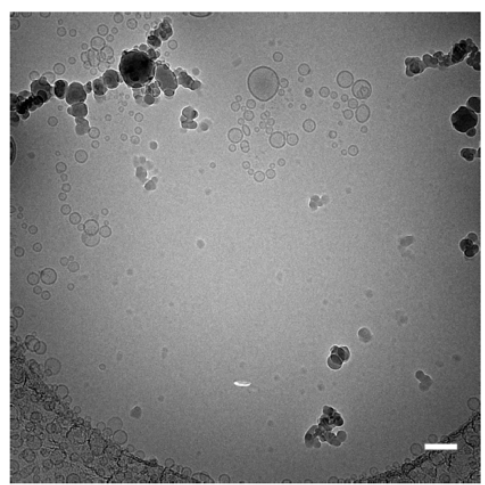

c)

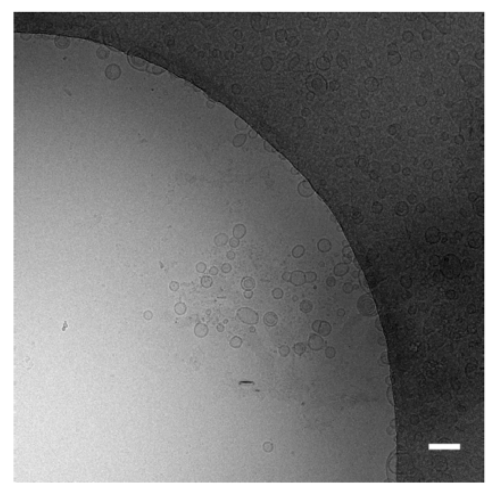

Figure 6.6: Representative overview cryo-electron microscopy images for (A) 0, (B) 20, and (C) 40 mol \% cholesterol in syb2 proteoliposomes. This figure was adapted from (Kreutzberger, et al., 2015).

Our result that increased cholesterol concentrations in the target and vesicle membranes promote direct full fusion vis-à-vis hemi-fusion indicates that cholesterol plays an important role in fusion pore opening. A large body of previous work has suggested that membrane components that alter the intrinsic spontaneous curvature of membranes are very important contributors of membrane fusion (Chernomordik \& Kozlov, 2008; Churchward, et al., 2008). Because cholesterol is well known to induce negative spontaneous curvature $\left(0.040 \AA^{-1}\right)$ in lipid bilayers (Chen \& Rand, 1997), we wanted to know if this property of cholesterol is critical for suppressing long-lived hemifusion and promoting direct full fusion events. $\alpha$-Tocopherol (vitamin $\mathrm{E}$ ) is an even stronger negative spontaneous curvature-promoting agent $\left(0.073 \AA^{-1}\right)$ in lipid bilayers than cholesterol (Churchward, et al., 2008; Brandford, et al., 2003). Because $\alpha$-tocopherol provides on a molar basis $\sim 1.82$ times more negative intrinsic spontaneous curvature to a lipid bilayer than cholesterol, we added 10 and $20 \mathrm{~mol} \% \alpha$-tocopherol to the supported 
a

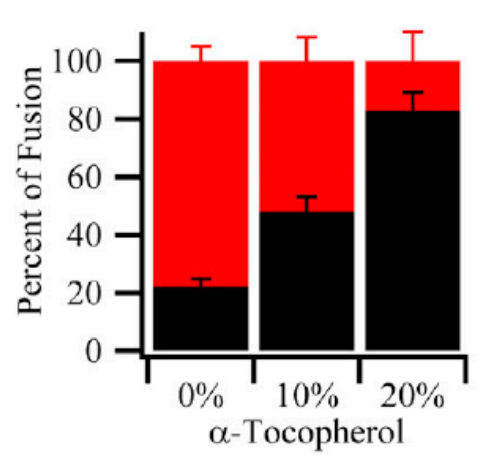

b

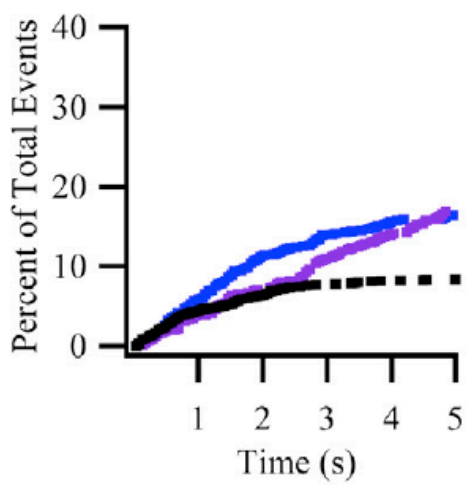

C

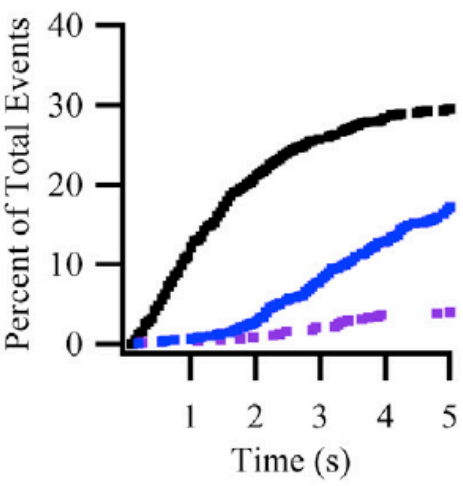

Figure 6.7: Summary of direct full fusion and hemi-fusion events obtained from single-vesicles fusion assay as a function of $\alpha$-tocopherol in the planar supported bilayer. Syb2 proteoliposomes were composed of bPC:bPE:bPS:chol:DiD (53:20:5:20:2) as in the experiments reported in Figure 6.2. (A) Relative fractions of direct full fusion (black) and hemifusion (red) of the total number of fusion events after $5 \mathrm{~s}$ (total numbers are shown in Table 6.5). The numerical values are $22 \pm 3 \%$, $50 \pm 5 \%$, and $79 \pm 5 \%$ direct full fusion and $78 \pm 6 \%, 50 \pm 3 \%$, and $21 \pm 3 \%$ for 0,10 , and $20 \mathrm{~mol}$ $\%$-tocopherol, respectively. (B) Cumulative distribution function of direct full fusion events normalized to the efficiencies of total docking events, which were $8 \pm 3 \%, 17 \pm 5$, and $15 \pm 5 \%$ for 0 (black), 10 (blue), and 20 (purple) mol \% $\alpha$-tocopherol, respectively. (C) Cumulative distribution function of hemi-fusion events normalized to the efficiencies of total docking events. Which were 29 $\pm 6 \%, 17 \pm 3 \%$, and $4 \pm 3 \%$ for 0 (black), 10 (blue), and 20 (purple) mol \% $\alpha$-tocopherol, respectively. This figure is adapted from (Kreutzberger, et al., 2015). 


\begin{tabular}{lcccc}
\hline$\alpha$-Tocopherol & $\begin{array}{c}\text { Direct Full } \\
\text { Fusion Events }\end{array}$ & $\begin{array}{c}\text { Hemifusion } \\
\text { Events }\end{array}$ & $\begin{array}{c}\text { Total } \\
\text { Events }\end{array}$ & $\begin{array}{c}\text { No. of Bilayers } \\
\text { Examined }\end{array}$ \\
\hline 0 & 92 & 332 & 1185 & 5 \\
10 & 131 & 134 & 890 & 5 \\
20 & 99 & 24 & 650 & 5 \\
\hline
\end{tabular}

Table 6.5: Summary of events at different $\alpha$-tocopherol concentrations in the planar supported bilayer. These data were used to generate Figure 6.7A and to normalize Figure 6.7B and $\mathrm{C}$. The error bars in Figure 5A represent the averages of all experiments under each condition. This data was adapted from (Kreutzberger, et al., 2015).

planar target membrane and used the single-vesicle fusion assay to measure the fusion of syb2 proteoliposomes that contained the standard $20 \mathrm{~mol} \%$ cholesterol. As with cholesterol, the proportions of direct full fusion to hemifusion increased as the amount of $\alpha$-tocopherol was about twice as powerful as cholesterol in favoring direct full fusion over long-lived hemifusion. Specifically, the direct full fusion fractions for $\alpha$-tocopherol over cholesterol were $\sim 1.4$ and $\sim 1.7$ at 10 and $20 \mathrm{~mol} \%$ of the two curvature agents, respectively. This result strongly suggests that the negative intrinsic spontaneous curvature of cholesterol is primarily responsible for its role in promoting direct full fusion. However, when the fusion kinetics are compared, the rates of hemifusion and direct full fusion were both slower in the presence of $\alpha$-tocopherol than in the presence of cholesterol (Figure 6.7 B and C), indicating that the more compact cholesterol fits better into transition states of fusion than the more flexible $\alpha$-tocopherol are not well described by the signal or two-consecutive-step kinetic models, and we did not attempt to fit these data. A summary of all recorded events under different conditions with $\alpha$-tocopherol is shown in Table 6.5. 


\subsection{Discussion}

Cholesterol is enriched in synaptic vesicle and presynaptic plasma membranes and modulates the localization and function of many secretory proteins including SNAREs (Lang, et al., 2001; Lang, 2007; Murray \& Tamm, 2009; Murray \& Tamm, 2011; Tong, et al., 2009; Mitter, et al., 2003) synaptophysin (Thiele, et al., 2000; Mitter, et al., 2003; Huttner \& Schmidt, 2000), synaptotagmins (Wan, et al., 2011; Lv, et al., 2008), and synaptic calcium channels (Taverna, et al., 2004; Kato, et al., 2003). Moreover, numerous reports have shown that cholesterol plays an important role in synaptic vesicle fusion (Zamir \& Charlton, 2006; Wasser, et al., 2007; Linetti, et al., 2010). However, the precise molecular mechanism, by which cholesterol acts on synaptic vesicle fusion, has not been elucidated. Because it is now well established that SNAREs are the central players in synaptic vesicle fusion, the role of cholesterol on the molecular mechanism of SNARE-mediated fusion is best studied in a reconstituted system. The reconstituted fast singlevesicle fusion system that we have developed is ideal for this purpose because it readily distinguishes between vesicle docking and fusion (Domanska, et al., 2009), their dependence on different lipid components (Domanska, et al., 2010), as well as its ability to distinguish between hemi- and full fusion (Kreutzberger, et al., 2015). This reconstituted system also recapitulates the fusion of purified synaptic vesicles (Kiessling, et al., 2013). Investigating the effects of cholesterol on fusion in a reconstituted system allowed us to directly assess its effects on SNARE-mediated membrane fusion without confounding additional effects on other proteins that may be present in cellular systems. As shown in Figure 6.3, we did not detect any significant differences in docking when cholesterol concentrations were changed in the target membrane and we do not anticipate that this would change by increasing cholesterol in the vesicle membrane. 
Using the reconstituted single-vesicle fusion assay, we found that hemi-fusion is the dominant mode of fusion at low cholesterol concentrations and that direct full fusion dominates at increased concentrations of cholesterol that best represent the physiological situations. Our results also show that physiological cholesterol is needed in both the syb2 vesicle and the tSNARE membrane to support direct full fusion. When cholesterol is lowered in either membrane, long-lived ( $>5 \mathrm{~s}$ ) hemi-fusion intermediates develop that only rarely proceed to a fully fused state. We thus consider most of these hemi-fused events as nonproductive side reactions that the cell avoids by incorporating enough cholesterol into the synaptic vesicle and presynaptic target membranes.

It is noteworthy that the fusion kinetics were best fit with a model of two consecutive steps for both the direct full fusion and the hemi-fusion cases. The kinetic fits of hemi-fusion and direct full fusion are both best characterized by a slow rate constant of $\sim 1 \mathrm{~s}^{-1}$ and a faster rate constant of $\sim 18 \mathrm{~s}^{-1}$ in direct full fusion or $\sim 8 \mathrm{~s}^{-1}$ in hemi-fusion (Table 6.2). We thus propose a model that combines the direct full fusion and hemi-fusion reactions into the following kinetic scheme:

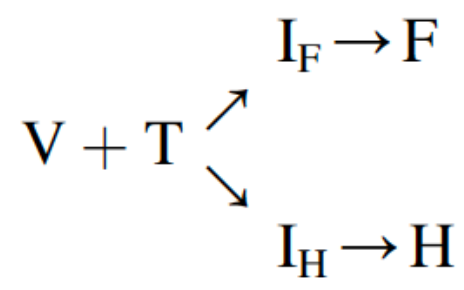

where vesicle $\mathrm{V}$ and target $\mathrm{T}$ membranes proceed to an intermediate I that then develops either into a full fusion pore $\mathrm{F}$ or a long-lived hemi-fusion state $\mathrm{H}$. Our experiments do not allow us to assign the faster or slower rates to the first or second steps in the above reaction scheme. 
However, irrespective of whether the process leading to the intermediates is represented by the faster or slower rates, the time constant for populating the intermediate is at least $100 \mathrm{~ms}$ and perhaps as long as $1 \mathrm{~s}$, i.e., long enough that we should see some lipid dye transfer if it had occurred during this time. Since our frame resolution in these experiments was $50 \mathrm{~ms}$ and we did not see any lipid transfer, the intermediates that we observed in either path cannot be a lipid stalk. More likely, it involves some rearrangement of the SNAREs that precedes the development of the stalk. However, once such a stalk has developed, it progresses directly into a full fusion pore at high cholesterol or into a dead-end hemi-fused state at low cholesterol on a timescale that must be faster than $50 \mathrm{~ms}$, i.e., the time resolution of our experiments.

(Chang, et al., 2009) measured the effects of cholesterol on fusion and SNARE assembly by bulk lipid mixing and a Förster resonance energy transfer assay, respectively. They concluded that cholesterol accelerated the kinetics of hemi-fusion but had only mild effects on fusion pore opening. These results are not directly comparable with ours because they measure the combination of docking and fusion on timescales of minutes and because they do not query the relative fractions of hemi- and full fusion. However, the different observations can be reconciled when one realizes that some fraction in the population of vesicles that are measured in the bulk fusion assay may proceed rapidly to hemi-fusion and eventually convert to full fusion after resting at a hemi-fused intermediate for a long time. In another study, (Tong, et al., 2009) showed that cholesterol in v-SNARE membrane caused a greater increase in the rate of lipid mixing than cholesterol in the t-SNARE membrane. The authors concluded that this increase was because of a structural rearrangement of syb2, but not the acceptor t-SNARE complex. We think 
that cholesterol might also change the partitioning of the N-terminal half of the SNARE motif of syb2 between the surface of the vesicle bilayer and the surrounding solution that we observed to be highly dependent on membrane curvature (Liang, et al., 2014). Based on fluorescence measurements, this partitioning is not expected to happen with the preassembled acceptor tSNARE complex in the target membrane (Liang, et al., 2013). It is also possible that the syb2 SNARE motif partitioning between the membrane surface and solution takes place on a timescale that would appear as two distinct populations in our fast single-vesicle fusion assay, but an average in the much slower bulk fusion assay. This timescale difference may well explain why the effects of cholesterol are symmetric in our single-vesicle fusion assay, but appear asymmetric in the bulk fusion assay.

Our result that $\alpha$-tocopherol causes very similar shifts in the relative proportions of direct full fusion and prolonged numerous reports have shown that hemi-fusion events at equivalent levels of induced negative spontaneous curvature argues strongly in favor of cholesterol causing this shift because of its ability to induce such curvature in lipid bilayers. Because $\alpha$-tocopherol does not order lipid, change their fluidity, or induce raft like domains in complex lipid mixtures, it is unlikely that these effects of cholesterol contributed significantly to the shift from hemi-fusion to the full fusion pathway. The requirement for negative intrinsic spontaneous curvature in membrane fusion is usually explained by the fact that this curvature stabilizes a lipid stalk intermediate on the way to forming a fusion pore (Chernomordik \& Kozlov, 2008; Yang \& Huang, 2002; Aeffner, et al., 2012). We consider it very likely that the lipid stalk promoted by cholesterol (and $\alpha$-tocopherol) represents a short-lived and kinetically invisible transition state 
on the path to open the fusion pore. Because the time resolution in our experiments was 50 to $100 \mathrm{~ms}$, the lifetime of the stalk must be less than $\sim 50 \mathrm{~ms}$ in our experiments. On the other hand, in the absence of cholesterol, the energy of the stalk transition state that leads to full fusion is high so that the membranes proceed to a different, relatively stable hemifusion state, which may not be an extended hemi-fusion diaphragm and which may or may not break to form a fusion pore at a later state (Chernomordik \& Kozlov, 2008; Chernomordik \& Kozlov, 2005). Because membranes with $\alpha$-tocopherol fuse more slowly than membranes with cholesterol, factors other than curvature also contribute to the energy barriers for transitioning directly into the fusion pore and the relatively stable hemifusion diaphragms. 


\section{Chapter 7: Complexin-1 interaction with plasma}

\section{membrane SNAREs}

Zdanowicz, R., A.J.B. Kreutzberger, B. Liang, V. Kiessling, L.K. Tamm, and D.S. Cafiso. (In

Press) Simultaneous Complexin Binding to Acceptor t-SNARE Complex and Membranes

Explains its Clamping Effect on v-SNARE Insertion and Fusion. Biophys $J$.

Fluorescent microscopy, fluorescent anisotropy, lipid mixing, and proteoliposomes preparations were performed by Alex Kreutzberger. EPR experiments were performed by Rafal Zdanowicz, and NMR experiments were performed by Binyong Liang.

\subsection{Summary}

Complexin-1 is a SNARE effector protein that decreases spontaneous neurotransmitter release and enhances evoked release. Complexin-1 binds to the fully assembled four helical neuronal SNARE core complex as revealed in competing molecular models derived from x-ray crystallography. Presently, it is unclear how complexin binding to the post-fusion complex accounts for its effects upon spontaneous and evoked release in vivo. Using a combination of spectroscopic and imaging methods, we characterize in molecular detail how complexin binds to the 1:1 plasma membrane t-SNARE complex of syntaxin-1a and SNAP-25 while simultaneously binding the lipid bilayer at both its $\mathrm{N}$ - and C-terminal ends. These interactions are cooperative and binding to the pre-fusion acceptor t-SNARE complex is stronger than to the post-fusion core 
complex. This complexin interaction reduces the affinity of synaptobrevin- 2 for the $1: 1$ complex for molecular models that account for the observed clamping effect of complexin beginning with the acceptor t-SNARE complex and the subsequent activation of the clamped complex by $\mathrm{Ca}^{2+}$ and synaptotagmin.

\subsection{Introduction}

In regulated exocytosis, an increase intracellular calcium triggers the fusion of secretory and synaptic vesicles with the plasma membrane thereby releasing the contents of the vesicle. This fusion process is controlled by a highly specialized fusion machinery, where SNARE proteins lie at the core of this machinery (Südhof, 2013; Tamm, et al., 2003; Wickner \& Schekman, 2008). Prior to fusion, the SNAREs are thought to be present in a partially assembled trans complex that is anchored through C-terminal transmembrane helical regions on synaptobrevin-2 and syntaxin-1a in the secretory vesicle and plasma membranes, respectively (Wickner \& Schekman, 2008). In this state, the vesicle and plasma membranes are in close proximity (Martens \& McMahon, 2008), and fusion occurs following the assembly of the SNAREs in an N- to Cterminal direction (Pobbati, et al., 2006). This highly exergonic process drives vesicle and plasma membranes into contact and overcomes the energy barriers to fusion (Jahn \& Scheller, 2006).

SNAREs are the minimal proteins required to reconstitute membrane fusion (Weber, et al., 1998); however, a number of additional proteins are required to regulate SNARE assembly, 
produce efficient fusion provide a response to calcium (Sorensen, 2009; Sudhof \& Rothman, 2009; Jahn \& Fasshauer, 2012; Sudhof, 2014). Complexin-1 is a highly-charged protein that is essential for calcium-mediated neurotransmission that appears to act by interacting with and regulating the SNAREs. Several studies have examined the role of complexin in membrane fusion and regulating the SNAREs. Several studies have examined the role of complexin in membrane fusion and yield seemingly contradictory results (Adberrahmani, et al., 2004; Brose, 2008; Huntwork \& Littleton, 2007; Itakura, et al., 1999; Roggero, et al., 2007; Tadokoro, et al., 2005). Complexin-1 exerts both positive and negative effects on vesicle exocytosis, facilitating synchronous neurotransmission while inhibiting spontaneous fusion events. Complexin-1 seems to act at a post-priming step of the fusion process, as neurons extracted from complexin-1, complexin-2 double knockout mice display a large reduction in calcium-triggered exocytosis, while still maintaining a wild-type number of primed vesicles (Reim, et al., 2001). Additionally, different regions within complexin appear to have different actions. The central $\alpha$-helix is critical for an inhibitory (clamping) effect of complexin on spontaneous neurotransmission, while the unstructured amino-terminus facilitates evoked exocytosis (Xue, et al., 2007). A long and flexible $\mathrm{C}$-terminal region is required for clamping and priming but not for calcium-triggering of fusion events (Kaeser-Woo, et al., 2012). In reconstituted systems, complexin reportedly modulates the docking and fusion events between membranes containing plasma membrane SNAREs (syntaxin-1a and SNAP-25) and the vesicle SNARE (synaptobrevin-2). Depending on the study, complexin has been reported to inhibit (Yoon, et al., 2008), facilitate (Diao, et al., 2013; Lai, et al., 2014), or not affect docking (Kim, et al., 2016) while suppressing spontaneous fusion and activating calcium-trigger fusion. 
Complexin-1 binds to the fully assembled post-fusion SNARE core complex (Ishizuka, et al., 1995) and a crystal structure of a fragment of complexin (residues 26-83) bound to this complex indicates that complexin associates as a fifth $\alpha$-helix (Chen, et al., 2002) in an antiparallel fashion between syntaxin-1a and synaptobrevin-2 (Figure 7.1). The interaction involves the central helix of complexin, leaving the more N-terminally localized accessory helix free while producing no significant conformational changes within the SNARE complex. In another crystal structure, the accessory helix bridges between two SNARE core complexes leading to the proposal that cross-linking of SNARE complexes accounts for the inhibitory activity of complexin (Kümmel, et al., 2011). Binding of complexin to defined prefusion SNARE complexes composed of syntaxin-1a and SNAP-25 (i.e. without synaptobrevin-2) has been difficult to establish, although such an interaction could be inferred from attenuated synaptobrevin-2 vesicle binding to t-SNARE vesicles that were preincubated with complexin (Gong, et al., 2016). Early work indicated that there were no interactions between complexin and the soluble, individual SNAREs or to plasma membrane t-SNARE complexes composed of syntaxin-1a and SNAP-25 (Pabst, et al., 2000). In one study using single molecule methods, complexin was reported to interact with and stabilize a 1:1 binary plasma membrane SNARE complex (Weninger, et al., 2008). However, the same group recently found that their SNARE complex preparations were heterogeneous resulting in unspecific sub-configurations unless a stabilizing synaptobrevin-2 peptide (residues 1-49) to assemble a well-defined SNARE acceptor complex (Choi, et al., 2016). Similarly, a different group demonstrate complexin binding to a plasma membrane SNARE complex by EPR and proposed that high micro molar concentrations of complexin might act to inhibit vesicle docking through a weak interaction with the plasma membrane SNAREs (Yoon, et al., 2008). However, a more recent study by this group claimed 
that complexin primes plasma membrane SNAREs by an unknown mechanism, and speculated that complexin might play a role in converting an inactive $2: 1$ syntaxin-1a/SNAP-25 complex to an active complex (Kim, et al., 2016).

Preparations of plasma membrane SNARES are frequently not homogenous and bulk assays may be complicated by signals from higher order oligomers of syntaxin-1a (Liang, et al., 2013) and 2:1 syntaxin-1a:SNAP-25 complexes that preferentially form and are known to be inefficient at fusion (Pobbati, et al., 2006). This may explain why complexin interactions with plasma membrane SNARES have been difficult to characterize. As a result, the mechanism by which complexin inhibits vesicle fusion in the absence of calcium and stimulates fusion in its presence remains highly debated, and no consensus regarding the importance of the observed complexinSNARE complex interactions has emerged (Trimbuch, et al., 2014; Krishnakumar, et al., 2015).

In order to better characterize the interaction of complexin with the prefusion acceptor plasma membrane SNARE complex, we took advantage of a new protocol that allows for the purification of monomeric syntaxin-1a (Liang, et al., 2013) and the assembly of a well-defined and highly active plasma membrane SNARE complex consisting of one copy of syntaxin-1a and one copy of SNAP-25 (Kreutzberger, et al., 2016). This 1:1 complex rapidly binds synaptobrevin-2 and promotes fusion at rates approaching that of an artificially stabilized plasma membrane SNARE complex (Pobbati, et al., 2006). In the present work, we use a range of biophysical methods to characterize the detailed molecular interactions of complexin to this new acceptor complex and we demonstrate that complexin strongly associates with the complex while 
simultaneously interacting with the lipid bilayer at both its $\mathrm{N}$ - and $\mathrm{C}$-terminal ends. The membrane interactions at both ends of complexin are membrane curvature dependent, confirming and extending previous work obtained in the absence of a plasma membrane SNARE complex (Gong, et al., 2016; Snead, et al., 2014), and both plasma membrane SNARE and membrane interactions act cooperatively to promote complexin association. Importantly the affinity of complexin for this complex is higher than for the ternary post-fusion core SNARE complex, suggesting that binding to the $1: 1$ acceptor complex is at least as important in regulating SNARE assembly as binding to the $1: 1: 1$ post fusion complex. We also find that binding of complexin to the binary plasma membrane SNARE complex dramatically reduces the rate of lipid mixing between reconstituted synaptobrevin-2 vesicles and plasma membrane SNARE vesicles and that this reduction is the result of a reduced affinity of synaptobrevin-2 for the plasma membrane SNARE complex. These data lead to a model for the inhibitory action of complexin on spontaneous fusion events that places this complexin/plasma membrane SNARE complex at the starting point for synchronous calcium and synaptotagmin triggered fusion events. 


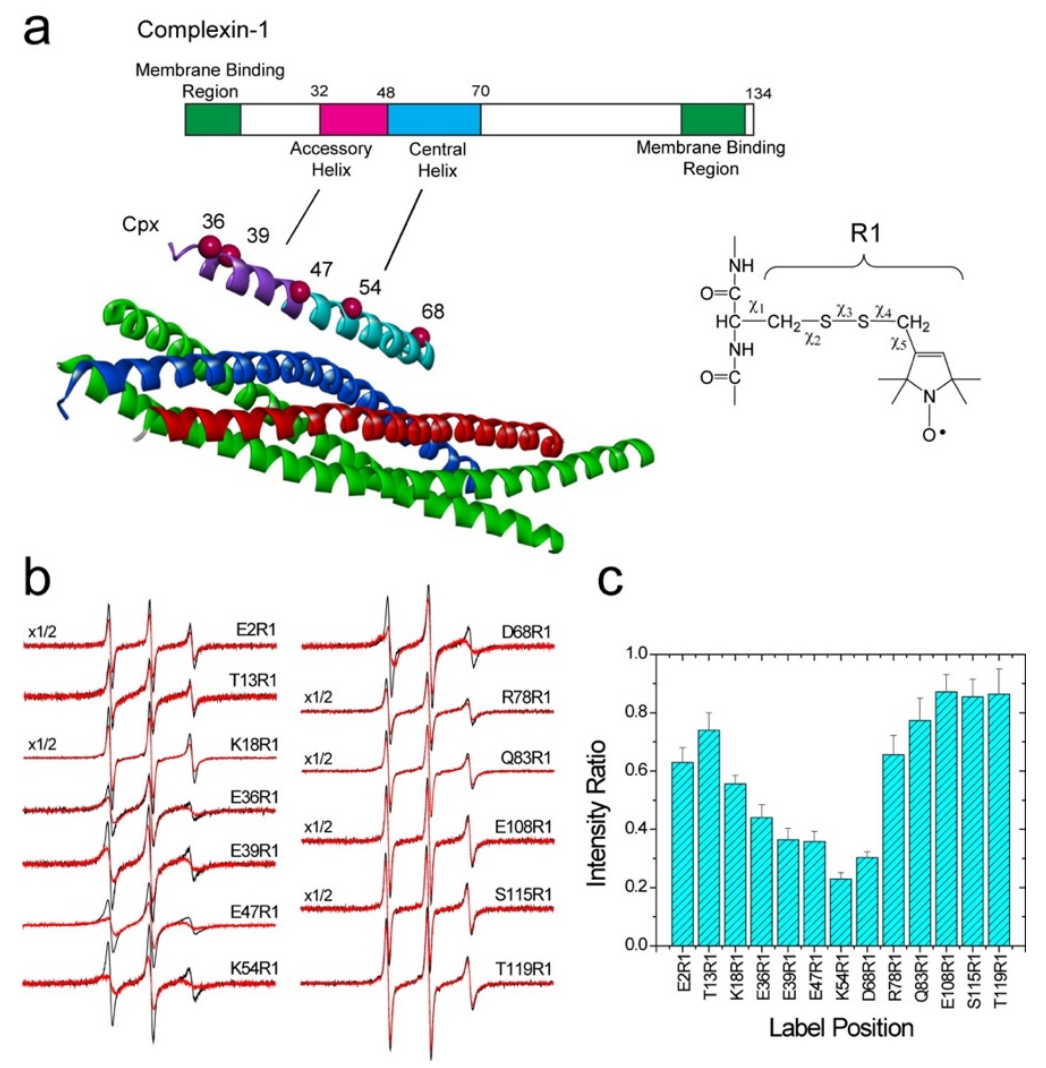

Figure 7.1: Complexin interacts with core SNARE complex: (a) complexin-1 contains regions reported to bind membranes and SNAREs. The crystal structure of a fragment of complexin bound to the core SNARE complex is shown (PDB ID: 1KIL) where SNAP-25, syntaxin-1a (183-288), and synaptobrevin-2 helices are indicated in green, red, and blue, respectively. In the present study, the spin labeled side chain $R 1$ was engineered into multiple sites in complexin, covering the $\mathrm{N}$ - and $\mathrm{C}$ termini, and the central helical region (magenta spheres indicate the position of several labeled sites on complexin in the accessory and central helices. (b) X-band CW-EPR spectra for 13 spin-labeled complexin mutants in solution (black trace) or incubated with the assembled core SNARE complex (red trace). Sites that interact with SNAREs exhibit a broadening of the EPR spectrum and a decrease in normalized amplitude. All spectra are100 Gauss scans with at least 10 averages taken. (c) Ratio of the normalized intensities for the high-field resonance line of each mutant in the presence/absence of the core SNARE complex. Error bars represent the uncertainty in the normalized intensity ratio due to normalization and measurement of peak-to-peak amplitudes. Experiments were performed by Rafal Zdanowicz. This figure was adapted from (Zdanowicz, et al., In Press). 


\subsection{Results}

Site directed spin labeling (SDSL) is well-suited to examine the molecular interactions of complexin to both soluble and membrane associated SNAREs, and the spin labeled sidechain R1

(Figure 7.1A) was introduced one at a time into full-length complexin by the reaction of the tiol specific MTSL spin label (1-oxyl-2,2,5,5-tetramethylpyrroline-3methyl) to single site directed cysteines.

As a starting point, we first probed the well-known interaction of complexin to an assembled 4helical soluble SNARE core complex (1:1:1 syntaxin-1a (180-253):SNAP-25:synaptobervin-2 (1-96) by EPR spectroscopy using 13 different spin labeled sites on complexin (Figure 1B). The addition of the soluble SNARE core complex resulted in a decrease in normalized amplitude of the complexin spectra, where positions in or near the central helix yielded the most dramatic line shape changes (e.g. E47R1, K54R1, and D68R1). Figure 1C plots these changes as a ratio of the normalized EPR intensity in the presence and absence of the 4-helix SNARE complex. Changes in EPR line shape may occur due to a reduction in the overall tumbling rate of complexin resulting from SNARE association; however, a comparison of the spectra in Figure 1B with those for complexin in sucrose (Figure 7.2) indicates that slowing the tumbling rate alone cannot account for the line shape changes at some sites. At several sites (E47R1, K54R1, and D68R1), the changes in normalized intensity reflect a reduction in local backbone dynamics that is due to the induction of stable secondary structure and a direct interactions with SNAREs. There is no indication in these spectra of tertiary contact of the label with the SNARE complex, consistent with the sites being outward facing in the complexin/SNARE complex structure (PDB ID:1KIL (Chen, et al., 2002). 

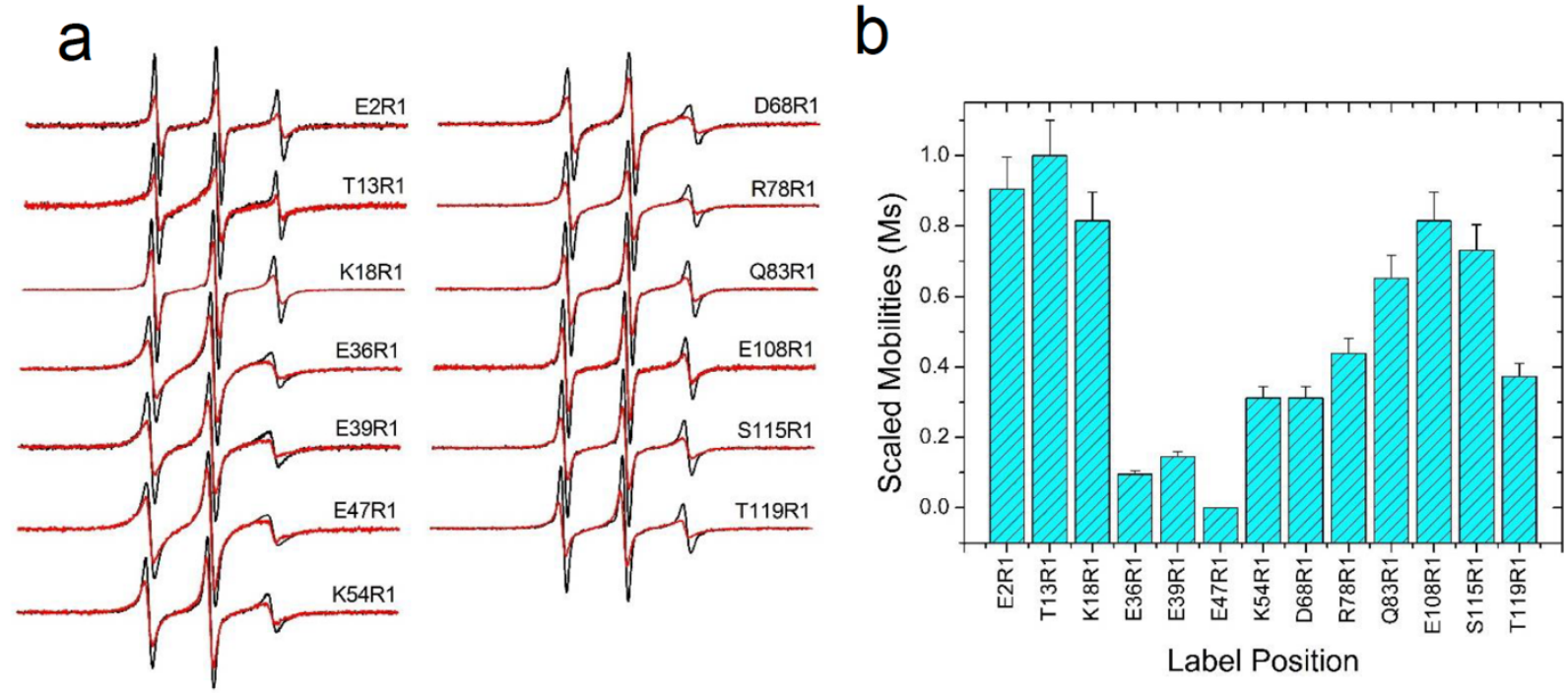

Figure 7.2: Addition of 30\% sucrose provides information on secondary structure within complexin-1 (a) X-band CW-EPR spectra of spin-labeled complexin-1 recorded in $30 \%$ sucrose to eliminated the effect of protein tumbling on spectral line shape. Black trace corresponds to complexin-1 in a physiological buffer (12 $\mathrm{mM} \mathrm{NaCl,} 139 \mathrm{mM} \mathrm{KCl,} 20 \mathrm{mM}$ MOPS, pH 7.4); the red trace corresponds to complexin in the physiological buffer supplied with $30 \%$ sucrose. (b) Scaled motilities (Ms) determined from spectra in panel (a) as described previously (Columbus \& Hubbell, 2002) range from 0 to 1 , where 0 represents the least mobile and 1 represents the most mobile spectra seen in complexin. Spin labels located within the accessory and central $\alpha$-helical domains are considerably less mobile than labels within the terminal domains indicating presence of a secondary structure. Data was collected by Rafal Zdanowicz. This figure was adapted from (Zdanowicz, et al., In Press). 

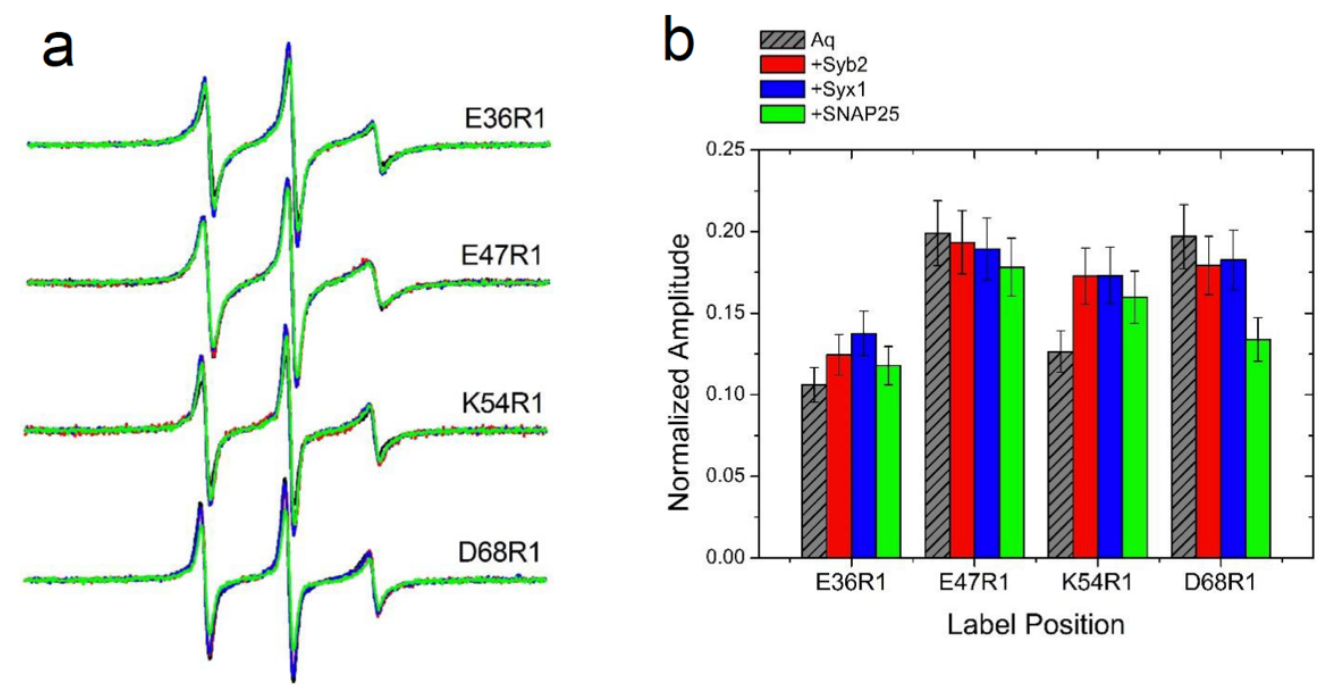

Figure 7.3: Complexin-1 does not interact with the individual, soluble SNAREs. (a) X-band CWEPR spectra of spin-labeled complexin recorded in the presence of individual SNARE proteins. Color-coding remains constant for each spin-labeled complexin: black, red, blue, and green traces correspond to free complexin, complexin mixed with synaptobrevin-2, syntaxin-1a (183-288), or SNAP-25, respectively. No spectral changes are observed indicating that there is no strong interaction between complexin and individual soluble SNAREs. (b) Normalized amplitudes of the central nitroxide resonance alone or in the presence of individual SNAREs. Complexin-1 and individual SNAREs were at a concentration of approximately $50 \mu M$. EPR spectra unless otherwise indicated are 100 Gauss scans. Experiments were performed by Rafal Zdanowicz. This figure was adapted from (Zdanowicz, et al., In Press). 
We also tested the binding of these complexin mutants to individual soluble SNAREs lacking their transmembrane segments. Upon the addition spin-labeled complexin to any of the three soluble individual SNAREs (synaptobrevin-2 (1-96), syntaxin-1a (180-253), or SNAP-25), no change in line shape was detected (see Figure 7.3), demonstrating that at the concentrations of complexin and SNAREs used here, no significant interaction takes place, consistent with previous findings (Pabst, et al., 2000).

The complexin/SNARE complex used for EPR (Figure 1) should resemble the previously published structure (PDB ID: 1KIL (Chen, et al., 2002)), and a comparison of the spectra in Figure 1B with this structure indicates that the resolved helical region of complexin in the crystal structure (sites 32 to 72 ) varies significantly in its backbone dynamics. Line shapes from sites E36R1 and E39R1 are consistent with helical structures (Columbus \& Hubbell, 2002; Columbus, et al., 2001) but indicate that the N-terminal end of this accessory helix is highly dynamic and is either fraying or undergoing significant rocking motions. This is consistent with the published structure (Chen, et al., 2002), where the resolved N-terminal end of complexin (residues 32-41) is not making efficient contact with the SNAREs. Near the C-terminal side of the central helix, D68R1 is also more dynamic than labels from the central region of this helix. These data indicate that sites from the C-terminal side of the accessory helix and N-terminal region of the central helix are largely responsible for the binding of complexin to the SNARE complex. EPR spectra from the N-terminal (E2R1, T13R1, and K18R1) and C-terminal (R78R1, Q83R1, E108R1, and T119R1) ends of full-length complexin indicate that these segments remain unstructured upon interaction with the soluble four-helical core SNARE complex. 
To determine whether complexin binds to a soluble binary acceptor plasma membrane SNARE complex, we used a fragment of syntaxin-1a (residues 191-253) lacking the transmembrane domain. This was purified and subsequently assembled with SNAP25 to form a binary complex as described (Kreutzberger, et al., 2016). We incubated this soluble binary complex with a complexin construct comprising the accessory and central helices, i.e. residues $26-83$, and measured their interaction by NMR. All resonances of free complexin could be assigned by hetero-nuclear experiments and yielded chemical shifts that were consistent with previous assignments (Pabst, et al., 2000), Figure 7.4A. After mixing with the soluble binary complex (sBC) in a 1:1 ration, many resonances of complexin became too broad to be observed (Figure 7.4B, C), particularly those towards the C-terminal end of the central helix. This indicates that these residues bind to a rather large complex possibly undergoing intermediate exchange on an approximately ms timescale. Significant signal attenuation was also observed for residues on the N-terminal side of central helix, whereas residues in the accessory helix were progressively less attenuated from the $\mathrm{C}$-terminal towards the $\mathrm{N}$-terminal end indicating a weaker interaction with the binary SNARE complex. In contrast, the extreme ends of the construct exhibit no signal attenuation, and are not likely interacting with the binary complex. When we changed the ratio of complexin (26-83):sBC from 1:1 to 2:1, 5:1, and 10:1, the respective attenuations decreased, but retained the same pattern with the intensities of the central helix residues showing higher degrees of attenuation compared to those of the accessory helix residues. The fact that significant attenuation persists even at these high molar ratios supports the notion of intermediate molecular exchange of the NMR time scale. The data also support the notion that the binding of complexin to the binary complex is initiated from the C-terminal half of the central helix and gradually extends towards both ends of complexin. Since we have not performed more detailed NMR 
exchange experiments on this system we cannot distinguish between thermodynamic and kinetic factors that might both contribute to the observed gradual binding patters.
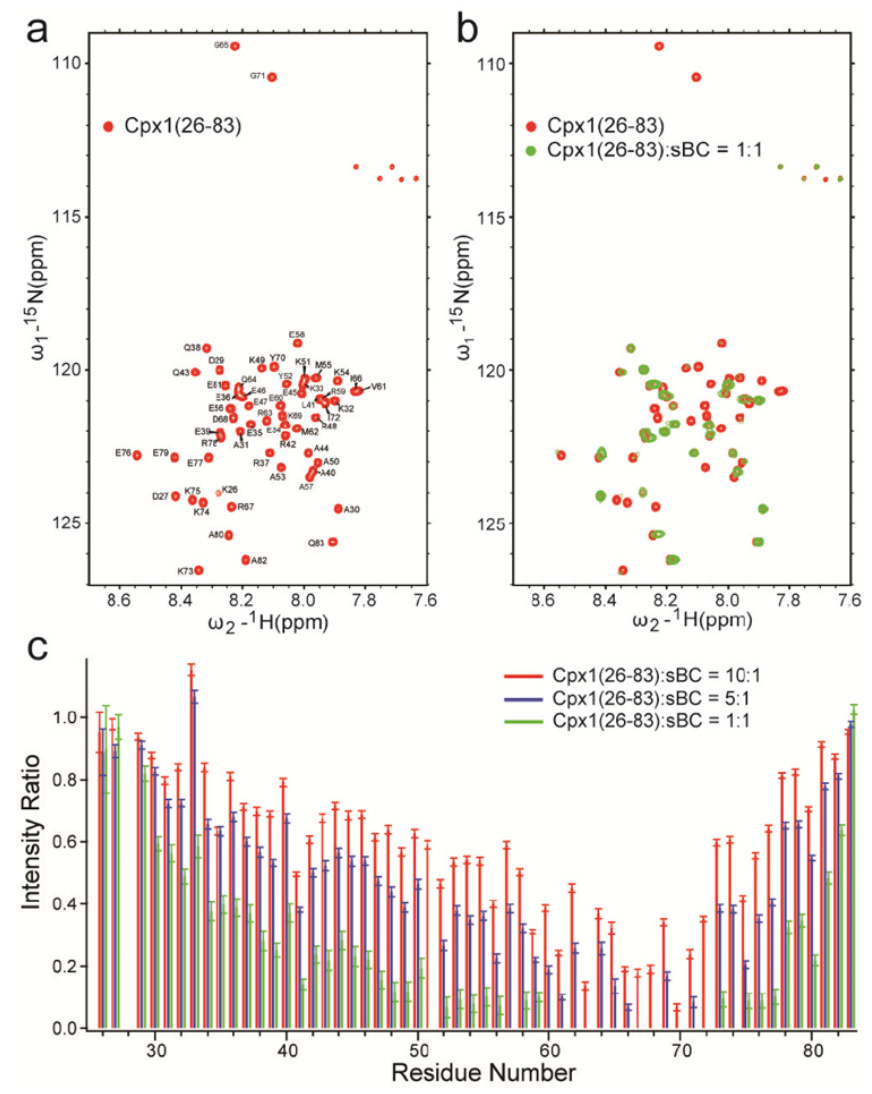

Figure 7.4: Complexin binds to soluble binary t-SNARE complex. (a) ${ }^{15} \mathrm{~N}-{ }^{1} \mathrm{H}$ HSQC spectrum of complexin (residues 26-83) in buffer. Assignments of backbone amides are denoted by one-letter amino acid abbreviations followed by their sequence numbers. (b) ${ }^{15} \mathrm{~N}-{ }^{1} \mathrm{H}$ HSQC spectrum of complexin (residues 26-83) mixed with an equimolar concentration of soluble binary complex (consisting of syntaxin-1a residues 191-253 and SNAP-25) (green), overlaid onto the HSQC spectrum of free complexin (residues 26-83): soluble binary complex, with ratios of 10:1, 5:1, and 1:1 represented in red, blue, and green bars, respectively. The intensity ratios are the intensities of complexin (residues 26-83)/soluble binary complex samples over the intensities of complexin (residues 26-83) alone and were calibrated using $\mathrm{N}$ - and $\mathrm{C}$-terminal residues to account for differences in sample concentrations. Only peaks with $\mathrm{S} / \mathrm{N}$ higher than 20 were analyzed, and error bars were propagated from $\mathrm{S} / \mathrm{N}$ levels of spectra and calibrated with redundant measurements. Experiments were performed by Binyong Liang. This figure was adapted from (Zdanowicz, et al., In Press). 
Complexin-1 has been reported to interact with lipid bilayers in its C-terminal region at two specific sites: A C-terminal motif and nearby amphipathic helix (Snead, et al., 2014; Seiler, et al., 2009). Binding of the N-terminal domain to membranes has also been reported (Gong, et al., 2016). Here, we used both NMR and continuous-wave EPR to examine these membrane interactions of full-length complexin and determine whether any structural changes occurred within complexin upon membrane binding.

Numerous backbone amide peaks undergo changes in chemical shift when phospholipidmimicking dodecylphosphocholine (DPC) micelles are added to complexin in solution. Based on resonance assignments obtained from respective NMR 3D backbone experiments, residues in the $\mathrm{N}$ - and C-terminal regions display large chemical shift differences, a few residues at the Cterminal end of central helix show moderate changes, and residues assigned to the accessory and central helix show almost no changes in chemical shift (Figure 7.5) In order to observe changes in a more lipid-like environment, we have also measured HSQC spectra of complexin in the presence of nanodics and liposomes. Shown in Figure 7.6 are the intensity ratios from HSQC spectra for full-length ${ }^{15} \mathrm{~N}$-labeled complexin in the presence and absence of vesicles or nanodiscs. Upon lipid binding, there is an increase in the rotational correlation time for segments directly interacting with the bilayer, and NMR line shapes in these regions broaden due to reduced averaging of chemical shift anisotropy and dipolar interactions. As seen in Figure 7.6A, the reductions in NMR peak intensity are not uniform but occur primarily for residues within either the $\mathrm{N}$ - and $\mathrm{C}$-terminal domains of complexin, while the more centrally localized sites 
show small or no levels of attenuation. The first 12 and the last 21 residues show the largest decreases, indicating that these sites are directly involved in simultaneous membrane binding.
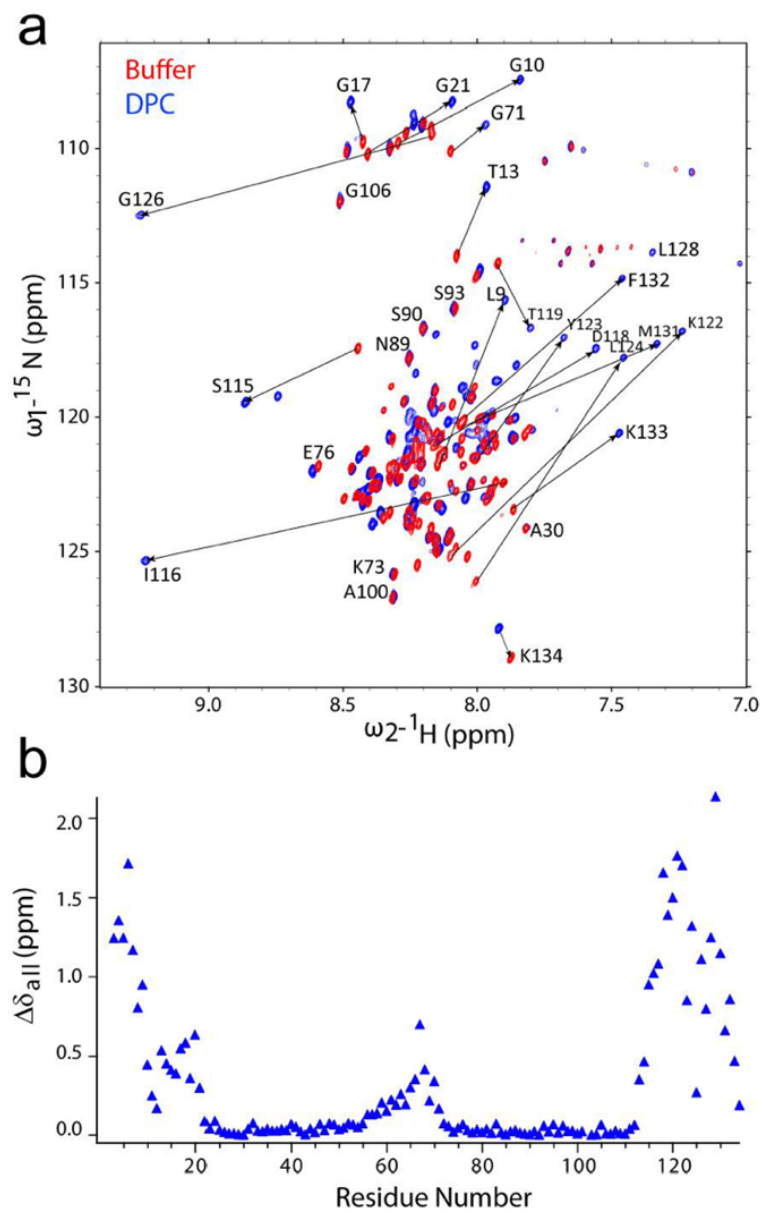

Figure 7.5: Complexin-1 changes conformation in a membrane mimetic environment. (a) Overlaid HSQC spectra of complexin in buffer (red) and with addition of $150 \mathrm{mM}$ DPC. Spectra were collected at $25^{\circ} \mathrm{C}$ and $800 \mathrm{MHz}$. (b) The combined chemical shift change $\Delta \delta_{\text {all }}(\mathrm{ppm})$ versus their respective residue numbers. Here, $\Delta \delta_{\text {all }}$ is defined as (Evenas, et al., 2001):

$\Delta \delta_{a l l}=\sqrt{\left(0.154 \cdot \Delta \delta_{N}\right)^{2}+\left(0.276 \cdot \Delta \delta_{C \alpha}\right)^{2}+\left(0.276 \cdot \Delta \delta_{C \beta}\right)^{2}+\left(0.341 \cdot \Delta \delta_{C o}\right)^{2}}$ eq. 7.1

Each $\Delta \delta$ value on the right side of the equation is the chemical shift difference of that particular nucleus. ${ }^{1} \mathrm{HN}$ chemical shifts were not included in this calculation since ${ }^{1} \mathrm{H}$ chemical shifts are more sensitive to surrounding local magnetic fields, and hence that value $\Delta \delta_{\text {all }}$ reflects principally changes in backbone $\phi / \psi$ angles. Experiments were performed by Binyong Liang. This figure was adapted from (Zdanowicz, et al., In Press). 
a

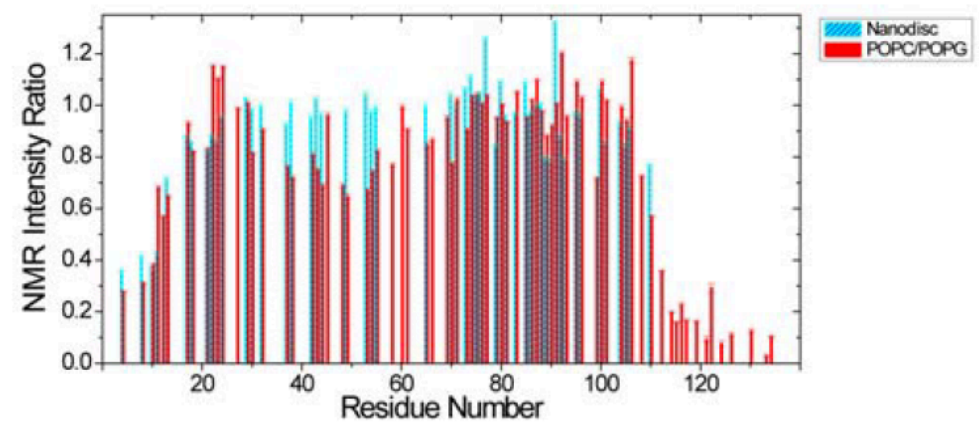

b

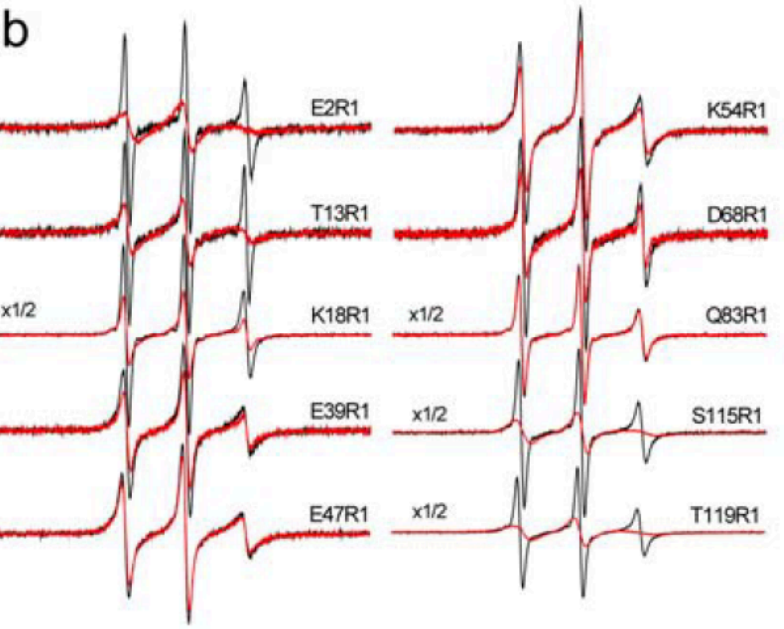

C

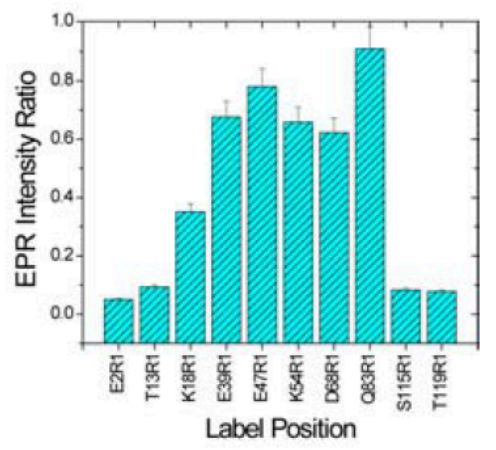

Figure 7.6: Complexin binds to membranes at both its $\mathrm{N}$ - and $\mathrm{C}$-terminal regions. (a) NMR intensity ratios of complexin bound to POPC nanodiscs (cyan bars) or POPC:POPG (90:10) bilayers (red bars). (b) EPR spectra in the absence (black trace) and presence (red trace) of POPC:POPS (70:30) liposomes and (c) intensity ratios for the high field resonance of spin labeled complexin mutants with/without POPC:POPS (70:30) liposomes. Error bars represent the uncertainty in the normalized intensity ratio due to normalized intensity ratio due to normalized intensity ratio due to normalization and measurement of peak-to-peak amplitudes. The data indicated the $\mathrm{N}$ - and $\mathrm{C}$ - termini of complexin are involved in direct membrane binding since the largest intensity drops both in the NMR data (a) and EPR data (b) are seen for the N- and Cterminal regions. Experiments were performed by Rafal Zdanowicz and Binyong Liang. This figure was adapted from (Zdanowicz, et al., In Press). 


\begin{tabular}{c|c|c|}
\hline Position & $\boldsymbol{\Phi}$ & Distance $(\AA)$ \\
\hline E2R1 & $-1.1 \pm 0.2$ & $1.7 \pm 0.9$ \\
\hline D68R1 & $-1.1 \pm 0.1$ & $1.6 \pm 0.5$ \\
\hline T119R1 & $-0.96 \pm 0.14$ & $2.2 \pm 0.6$ \\
\hline
\end{tabular}

Table 7.1: Depth parameters, $\Phi$, and distances from the phospholipid phosphate plane determined for R1-labeled complexin by power saturation of the EPR spectrum. Positive values of the membrane depth $(\AA)$ indicate the nitroxide $R 1$ side chain is positioned on the hydrocarbon side of the phosphate plane within the lipid bilayer. The errors in the depth are calculated based on standard deviation of $\Phi$. Experiments were performed by Rafal Zdanowicz. This figure was adapted from (Zdanowicz, et al., In Press).

To further test this result, EPR spectra (Figure 7.6B) were recorded for ten spin-labeled mutants covering the complexin sequence in the presence and absence of POPC:POPS (70:30 mol\%) small unilamellar vesicles (SUVs). The normalized intensity ratios for the high-field resonance peaks of complexin in the absence/presence of liposomes are shown in Figure 7.6C. Upon membrane association, the EPR line shapes broaden, due to a combination of changes in backbone dynamics and a change in the selection of R1 rotamers that occurs upon insertion of the spin label into the membrane (Freed, et al., 2011; Kroncke, et al., 2010). The data indicate that both the $\mathrm{N}$ - and C-terminal regions of complexin contact the membrane interface, consistent with the NMR result. Comlexin-E2R1, T13R1, K18R1, S115R1, and T119R1 yield highly averaged EPR line shapes in the absence of membrane, but upon addition of SUVs, all of these sites exhibit a significant increase in spectral linewidth and a drop-in signal amplitude. Smaller 
changes are observed for complexin-D68R1 and perhaps complexin-K54R1, indicating that they may also interact with the lipid interface. Other sites in complexin yield no or small changes in EPR line shapes in the presence of lipid bilayers.

To determine the position of complexin relative to the membrane interface, we power-saturated EPR spectra from sites E2R1, D68R1, and T119R1 and obtained membrane depth parameters. The membrane depth parameters, $\Phi$, and position of the spin label relative to the lipid phosphate plan are shown in Table 7.1. Values obtained for all three complexin mutants: E2R1, D68R1, and T1119R1 place the labels at a depth of 1.6-2.2 $\AA$ from the phosphate plane. The labels do not reach the acyl chain region of the bilayer, and the data demonstrate that complexin is peripherally associated with the membrane interface.

It has been previously reported that the binding of the C-terminus of complexin to lipid vesicles depends on membrane curvature (Gong, et al., 2016; Snead, et al., 2014). To compare the curvature-dependent binding at both ends of full-length complexin, we determined the equilibrium binding affinities of complexin to liposomes of different sizes, $\sim 100, \sim 50, \sim 25 \mathrm{~nm}$ in diameter (Figure 7.7) by EPR spectroscopy using both complexin-E2R1 and T119R1. As seen in Figures 7.8B and $\mathbf{C}$, labels significantly stronger binding to the smallest and most curved vesicles. The $\mathrm{C}$-terminal site, significantly stronger binding to the smallest and most curved vesicles. The C-terminal site, complexin-T119R1 (Figure 7.8C and Table 7.2) displays an 80fold difference in affinity between the largest and smallest vesicles, corresponding to a free 
energy difference of about $2.6 \mathrm{kcal} / \mathrm{mol}$. The $\mathrm{N}$-terminus shows a similar affinity to the smallest vesicles and a 40-fold weaker affinity towards the largest vesicles (Figure 7.8B and Table 7.2). The result for the C-terminus is generally consistent with previous findings (Gong, et al., 2016; Snead, et al., 2014) and the data for the N-terminus indicates that it has a similar curvaturedependent binding. We also tested to see if the $\mathrm{N}$-terminal and C-terminal interactions of complexin were cooperative, by measuring the affinity at site T119R1 when the $\mathrm{N}$-terminus was truncated (complexin residues 26-134). As seen in Figure 7.8D, when the N-terminus is truncated the membrane affinity of the C-terminal end is not significantly changed. Thus, both ends of complexin bind to membranes in a curvature-dependent manner and act independently of each other. This may be a result of long disordered segments being present between the $\mathrm{N}$ - and C-terminal regions of complexin.
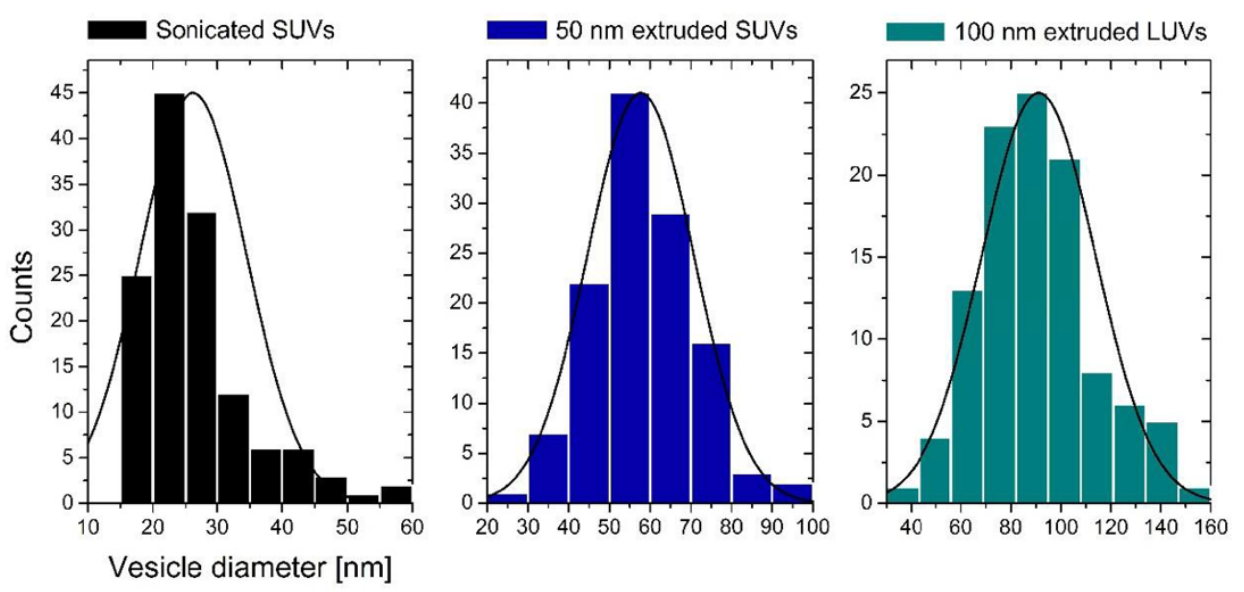

Figure 7.7: The size distribution of liposomes generated by sonication or extrusion through polycarbonate membranes. The vesicle diameters were measured from cryo-electron microscopy images of the vesicle preparations. Experiments were performed by Rafal Zdanowicz. This figure was adapted from (Zdanowicz, et al., In Press). 

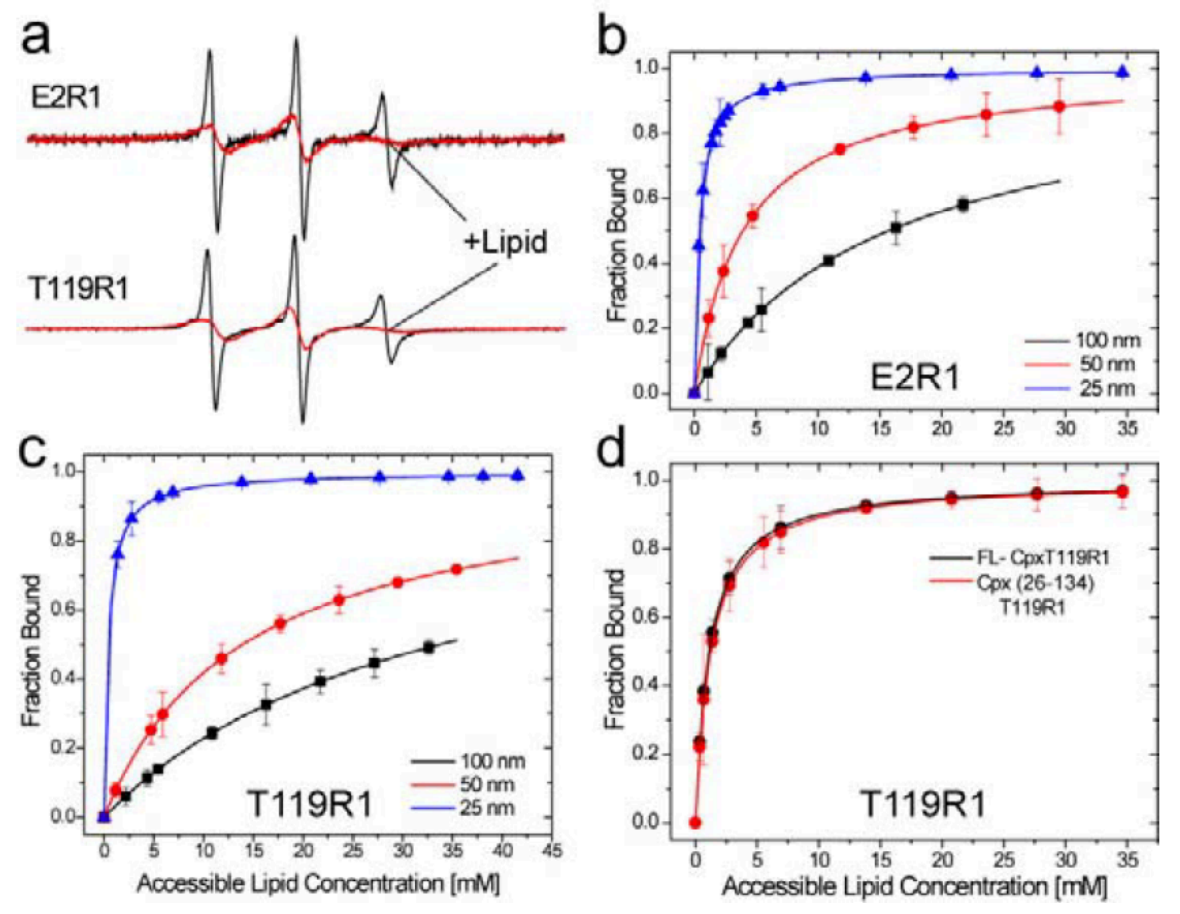

Figure 7.8: Complexin senses membrane curvature. (a) EPR spectra of E2R1 and T1119R1 show dramatic changes in line shape upon membrane binding, and these changes were used to determine complexin membrane affinity. (b) E2R1 and (c) T119R1 with unilamellar vesicles of varied size $(\sim 100, \sim 50$, and $\sim 25 \mathrm{~nm}$ in diameter) yield the fraction of membrane bound complexin as a function of accessible lipid. These data were fit (solid lines) to a simplex binding isotherm to yield the membrane binding partition coefficient shown in Table 7.2. In (d) the binding of complexin199R1 in full-length complexin was compared with that for an $\mathrm{N}$-terminally truncated version of complexin (residues 26-134) to small $25 \mathrm{~nm}$ diameter vesicles, and indicate the $\mathrm{N}$ - and C-terminal regions act independently. All data points are averages of three titrations. Error bars represent deviations from the best fit. Experiments were performed by Rafal Zdanowicz. This figure was adapted from (Zdanowicz, et al., In Press). 


\begin{tabular}{|c|c|c|c|c|}
\hline & $\begin{array}{l}\text { E2R1 partition } \\
\text { coefficient }\left[\mathbf{M}^{-1}\right]\end{array}$ & $\begin{array}{l}\text { E2R1 lipid binding } \\
\text { affinity [mM] }\end{array}$ & $\begin{array}{l}\text { T119R1 partition } \\
\text { coefficient }\left[\mathrm{M}^{-1}\right]\end{array}$ & $\begin{array}{l}\text { T119R1 lipid binding } \\
\text { affinity [mM] }\end{array}$ \\
\hline $\begin{array}{l}92 \pm 23 \mathrm{~nm} \\
\text { LUVs }\end{array}$ & $64 \pm 6$ & $16 \pm 1$ & $30 \pm 2$ & $34 \pm 2$ \\
\hline $\begin{array}{l}58 \pm 13 \mathrm{~nm} \\
\text { SUVs }\end{array}$ & $260 \pm 31$ & $3.9 \pm 0.4$ & $72 \pm 15$ & $14 \pm 2.5$ \\
\hline $26 \pm 8 \mathrm{~nm} \mathrm{SUVs}$ & $2,400 \pm 180$ & $0.42 \pm 0.03$ & $2,300 \pm 210$ & $0.43 \pm 0.04$ \\
\hline
\end{tabular}

Table 7.2: Partition coefficients and binding affinities for the binding of complexin-E2R1 and complexin-T119R1 to vesicles of different sizes. Phase partition coefficients and the respective binding affinities were determined for complexin-1 incubated with liposomes. The lipid binding affinities are equal to $\mathrm{K}^{-1}$ in $\mathrm{mM}$, where $\mathrm{K}$ is the reciprocal molar partition coefficient. The lipid binding affinity, $\mathrm{K}^{-1}$, represents the concentration of accessible lipid (in $\mathrm{mM}$ ) that produces halfmaximal membrane binding of complexin. Experiments were performed by Rafal Zdanowicz. This figure was adapted from (Zdanowicz, et al., In Press).

To determine whether complexin is capable of simultaneous interactions with both membranes and membrane-embedded SNAREs, spin-labeled full length complexin was added to membranereconstituted syntaxin-1a (183-288), a dodecylated version of SNAP-25a (d-SNAP-25), or a reconstituted 1:1 syntaxin-1a:d-SNAP-25 t-SNARE complex. The d-SNAP-25 was produced by chemical alkylation of the four native cysteines closely resembling the palmitoylated form of the protein, which is predominant in neurons or SNAP-25 expressing insect cells (Foley, et al., 2012; Viet, et al., 1996). This 1:1 syntaxin-1a:d-SNAP-25 acceptor complex is functionally similar to an artificial $\Delta \mathrm{N}$ complex that produces fast and efficient fusion events (Kreutzberger, et al., 2016), but it does not require the short synaptobrevin-2 (49-96) peptide needed to stabilize syntaxin-1a and SNAP-25 into a 1:1 state (Pobbati, et al., 2006; Domanska, et al., 2009). 


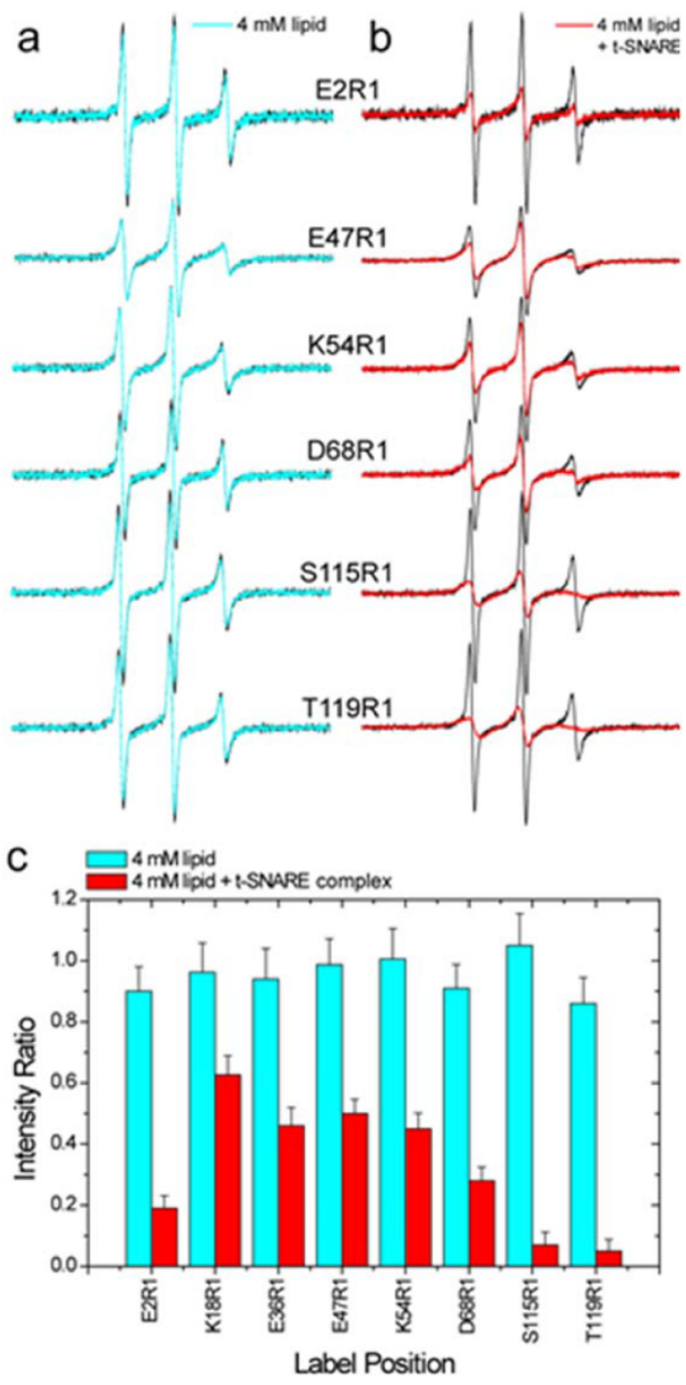

Figure 7.9: Membranes with the reconstituted acceptor t-SNARE complex enhance the membrane binding of complexin. EPR spectra from different spin-labeled complexin mutants in solution (black traces) or incubated with either (a) liposomes at $4 \mathrm{mM}$ lipid (70:30 POPC:POPS) (cyan trace) or (b) proteoliposomes with $4 \mathrm{mM}$ lipid and t-SNARE complex (70:30 POPC:POPS) at a protein:lipid ratio of 1:100 (red traces). (c) Normalized intensity ratios for spin-labeled complexin in the presence/absence of liposomes (4 mM lipid) (cyan bars) or in the presence/absence of proteoliposomes with t-SNAREs (red bars). The ratios are determined from normalized intensities measured from high-field nitroxide resonance line $\left(M_{1}=-1\right)$. The error range represents the uncertainty in the determination of the ratio. Protein free liposomes were prepared by dialysis in an identical manner to that for the proteoliposomes. Experiments were performed by Rafal Zdanowicz. This figure was adapted from (Zdanowicz, et al., In Press). 


\begin{tabular}{c|c|c} 
CpxT119R1 with: & Partition Coefficient $\left[\mathbf{M}^{-1}\right]$ & $\begin{array}{c}\text { Lipid Binding Affinity } \\
{[\mathbf{m M}]}\end{array}$ \\
\hline Liposomes $\uparrow$ (POPC/POPS) & $330 \pm 30$ & $3.0 \pm 0.3$ \\
\hline Syx proteoliposomes & $290 \pm 30$ & $3.5 \pm 0.3$ \\
\hline dSNAP25 proteoliposomes & $1,460 \pm 190$ & $0.69 \pm 0.08$ \\
\hline $\begin{array}{c}\text { Syx:dSNAP25 } \\
\text { proteoliposomes }\end{array}$ & $6,300 \pm 900$ & $0.16 \pm 0.02$ \\
\hline $\begin{array}{c}\text { Syx:SNAP25:Syb2 } \\
\text { proteoliposomes }\end{array}$ & $2100 \pm 240$ & $0.50 \pm 0.06$ \\
\hline
\end{tabular}

Table 7.3: Partition coefficients and binding affinities for the binding of complexin to membranes in the presence and absence of reconstituted SNAREs. Phase partition coefficients and the respective binding affinities were determined for complexin-T119R1 incubated with SNARE proteoliposomes. The lipid binding affinities are equal to $\mathrm{K}^{-1}$ in $\mathrm{mM}$, where $\mathrm{K}$ is the reciprocal molar partition coefficient. The lipid binding affinity, $\mathrm{K}^{-1}$, represents the concentration of accessible lipid (in $\mathrm{mM}$ ) that produces half-maximal membrane and SNARE binding of complexin. The protein:lipid ratio of SNARE containing proteoliposomes is 1:100. These protein-free liposomes are prepared by dialysis from cholate, as are the proteoliposomes; as a result, the binding affinities of complexinT119R1 are not directly comparable to those for the extruded liposomes in Table 7.2. Experiments were performed by Rafal Zdanowicz. This figure was adapted from (Zdanowicz, et al., In Press). 
Shown in Figure 7.9 are EPR spectra from six R1 labeled sites within complexin that interact with SNAREs or membranes (Figures 7.1, 7.4, and 7.6). In Figure 7.9A, $30 \mu \mathrm{M}$ complexin is added to protein-free liposomes prepared by dialysis at a lipid concentration of $4 \mathrm{mM}$. For these larger liposomes, $4 \mathrm{mM}$ is not a lipid concentration high enough to bind a significant fraction of complexin. As expected, no significant interaction is detected at any complexin site. In Figure 7.9B, these same complexin mutants are added to the binary plasma membrane SNARE complex (syntaxin-1a:dSNAP-25) reconstituted into membranes at this same lipid concentration (4 mM) and a protein concentration of $40 \mu \mathrm{M}$. In contrast to Figure 7.9A, significant line shape broadening is seen both at the $\mathrm{N}$ - and C-termini (positions 2,115, 119), which interact with membranes, and within the central helical region (positions 47, 54, 68), which interact with the core SNARE complex (Figure 7.1) (Chen, et al., 2002; Kümmel, et al., 2011) or the soluble 1:1 plasma membrane SNARE complex (Figure 7.4). The relative normalized EPR intensity changes upon the addition of pure lipid membranes or the membrane-reconstituted acceptor plasma membrane SNARE complex are shown in Figure 9C. The observed line shape changes at the N-and C-termini seen in Figure 9B resemble those that take place at sites on membranes (Figure 7.6). The line shapes for sites near the central helix of complexin (sites 46, 54, and 68) resemble those seen with the SNARE core complex (Figure 7.1) and are consistent with the R1 label at exposed helical sites (Columbus \& Hubbell, 2002). No significant changes in line shape occur when labeled complexin is added to membranes containing only reconstituted syntaxin-1a; however, line shape changes are observed when complexin is added to membranes containing only d-SNAP- 25 at the same protein:lipid ratio as in the experiments with the binary plasma membrane SNARE complex. These changes yield a different pattern of contact than that seen with the plasma membrane SNARE complex and were not further investigated (Figure 7.10). 


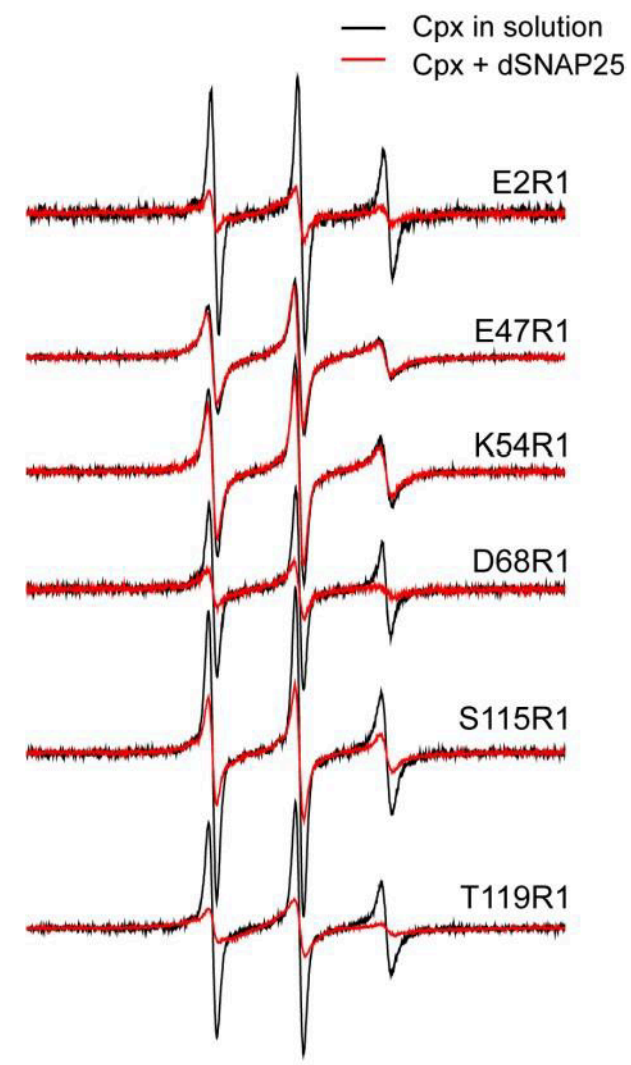

Figure 7.10: EPR spectra for spin labeled complexin in solution (black trace) or in the presence of membranes (red trace) with 4 mM d-SNAP-25 proteoliposomes (70:30 POPC:POPS) at a protein:lipid ratio of (1:100). This lipid concentration alone is insufficient to bind complexin. Experiments were performed by Rafal Zdanowicz. This figure was adapted from (Zdanowicz, et al., In Press). 
Figure 7.9 demonstrates that the presence of the binary SNARE promotes complexin binding under conditions where lipid interactions alone are insufficient to bind complexin. As a result, simultaneous membrane-SNARE interactions are contributing to the free energy of complexin binding, and the EPR spectra data provide strong evidence that complexin is simultaneously contacting both membranes and the 1:1 t-SNARE complex.

To estimate the contribution that the SNAREs make to the membrane binding of complexin, we used site T119R1 to measure complexin membrane affinity by titrating increasing lipid concentrations into a sample having a fixed concentration of labeled complexin. As seen in Figure 7.11A and Table 7.3, the affinity of complexin to membranes containing syntaxin-1a is not significantly different from lipid alone; however, the presence of the 1:1 plasma membrane SNARE complex dramatically increases the affinity of complexin. The partition coefficient is increased by 19-fold, indicating that interaction of complexin with the membrane-associated plasma membrane SNARE complex increases the free energy of membrane association by about $1.8 \mathrm{kcal} / \mathrm{mole}$. Complexin also binds the assembled membrane-reconstituted 1:1:1 SNARE core complex, but with a 3-fold weaker affinity than the membrane-associated binary SNARE complex.

To confirm these results using a different approach, we measured the fluorescence anisotropy of Alexa 546-labeled complexin in the presence of liposomes without or with syntaxin-1a, dSNAP-25, or the syntaxin-1a:d-SNAP-25 acceptor SNARE complex. As seen in Figure 7.11B, 
there is little anisotropy change in the presence of the protein-free liposomes, slight increases in the presence of syntaxin-1a, larger changes with d-SNAP-25 and the membrane-reconstituted SNARE core complex, and the largest changes with the acceptor SNARE complex. This roughly follows the order of membrane affinities measured by EPR. It is also important to note that there is a lower affinity of complexin to the artificial $\Delta \mathrm{N}$ complex (Pobbati, et al., 2006), and almost no binding to a syntaxin-1a:SNAP-25 complex assembled in CHAPS. The lower affinity to the $\Delta \mathrm{N}$ complex suggests that synaptobrevin-2 (49-96) competes with the complexin binding site on the plasma membrane SNAREs, and the complex assembled in CHAPS has a 2:1 syntaxin1a:SNAP-25 stoichiometry (Kreutzberger, et al., 2016).

We also employed Total Internal Reflection Fluorescence (TIRF) microscopy to estimate the binding of complexin to planar supported bilayers in the presence and absence of SNAREs, where plasma membrane SNARE complexes were incorporated into supported bilayers. Figure 7.11C shows the binding of fluorescently labeled complexin as a function of concentration to membranes with and without plasma membrane SNAREs. Complexin-1 displays only a weak interaction with the protein-free membrane, whereas the presence of the binary SNARE complex causes a dramatic increase in this interaction. As seen in Figure 7.11C, about $2 \mu \mathrm{M}$ complexin is sufficient to saturate the planar supported bilayer as higher complexin concentrations do not significantly increase the measured fluorescence. This result is also consistent with the EPR result, showing the SNARE and membrane interactions act cooperatively to promote complexin binding. 


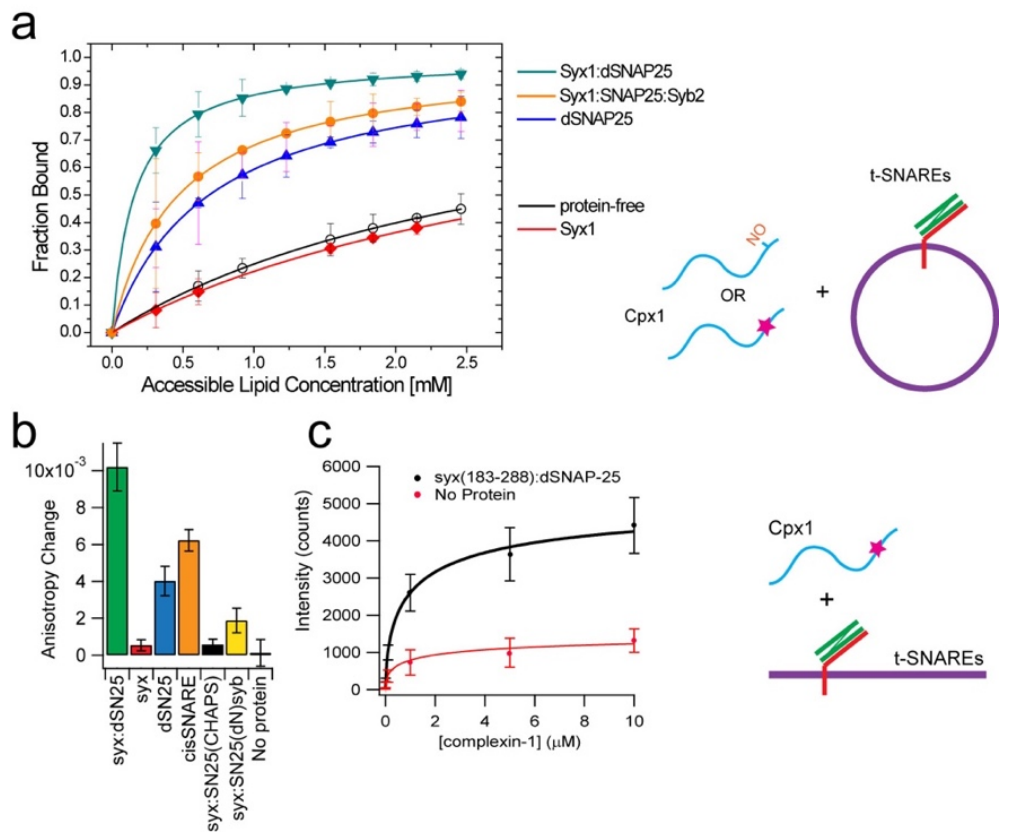

Figure 7.11: Complexin strongly binds to proteoliposomes with reconstituted acceptor t-SNARE complexes. (a) Binding curves for complexin using the spin-labeled mutant CpxT119R1 by recording EPR spectra with increasing concentrations of proteoliposomes that were either protein-free (black trace) or reconstituted with syntaxin-1a (red trace), dSNAP25 (blue trace), the Syx:dSNAP25 tSNARE complex (green trace), or the assembled cis-SNARE complex (orange trace). All proteins were present at a protein-lipid ratio of 1:100. Points are averages of three titrations, and errors represent deviations from the best fit. (b) fluorescence anisotropy measurements are consistent with EPR, where fluorophore-labeled complexin was mixed with either protein-free liposomes, dSNAP25, syntaxin-1a, or t-SNARE-containing proteoliposomes composed of 70:30 POPC:POPS at a lipid:protein ratio of 400:1. The effect of the interaction was quantified and plotted as a change in fluorescence anisotropy relative to free complexin. Anisotropy changes are the average of 4 experiments, and the error bars represent standard errors. (c) The binding of complexin to planar supported bilayers was measured with TIRF microscopy. Increasing concentrations of labeled Cpx were titrated into the planar bilayer and increases in fluorescence in the TIRF field were monitored. The fluorescence as a function of complexin concentration is shown for protein-free planar bilayers (70:30 POPC:POPS) (red trace) and for planar bilayers (70:30 POPC:POPS) reconstituted with syntaxin-1:dSNAP25 (lipid:protein of 3000) (black traces). For each condition, values are averages from three separate bilayer preparations. Error bars are standard errors. EPR experiments in (a) were performed by Rafal Zdanowicz. This figure was adapted from (Zdanowicz, et al., In Press). 
As reported previously, the reconstituted syntaxin-1a:d-SNAP-25 acceptor SNARE complex used here promotes fast membrane fusion with synaptobrevin-2-containing vesicles (Kreutzberger, et al., 2016). Here, we tested the effect of complexin on fusion in this system. We first employed a lipid mixing assay for fusion where vesicles containing synaptobrevin-2 or the binary plasma membrane SNARE complex are mixed at a 1:1 ratio in the presence of complexin. When lipid mixing occurs, rhodamine and 7-nitrobenzofurazan (NBD) in the vesicle SNARE membrane are diluted and the NBD fluorescence increases. As seen in Figure 7.12A, complexin has a potent inhibitory effect on lipid mixing in the absence of calcium. With no complexin added, proteoliposomes fused efficiently, whereas introduction of $8 \mu \mathrm{M}$ complexin blocks fusion almost entirely (Figure 7.12B), reaching only 5\% of the lipid mixing observed in the absence of complexin. These data demonstrate that complexin has an inhibitory effect on lipid mixing in the absence of calcium in this system.

The fusion rates observed in this lipid mixing assay may be due to the effects of complexin upon membrane docking or upon fusion itself. To distinguish between docking and fusion, we added complexin to planar supported bilayers containing reconstituted 1:1 acceptor plasma membrane SNARE complexes without the addition of calcium. Fluorophore-labeled synaptobrevin-2 proteoliposomes were added and the number of bound vesicles per $\mu \mathrm{m}^{2}$ area of the supported bilayer was determined at each time point using TIRF microscopy. Figure 7.12C shows the cumulated docking events that occur as a function of time. Synaptotavein-2 proteoliposomes bind to syntaxin-1a/SNAP-25 SNARE complexes reconstituted into the planar bilayers reaching a density of approximately 6 vesicles $/ \mu \mathrm{m}^{2}$ in a period of $1,200 \mathrm{~s}$. As seen in Figure 7.12C and D, 
complexin inhibits vesicle docking, where the inhibition saturates at about $2 \mu \mathrm{M}$ complexin. It is important to note that while complexin inhibits docking, it does not saturates at about $2 \mu \mathrm{M}$ complexin. It is important to note that while complexin inhibits docking, it does not completely prevent docking even at a concentration of $2 \mu \mathrm{M}$. These data indicate that the reduction in docked synaptobrevin-2 vesicles in the presence of complexin is due to a reduction in affinity of synaptobrevin-2 to the acceptor plasma membrane SNARE complex. To directly test this result, we measured the binding of a soluble fragment of synaptobrevin-2 (residues 1-96, with residues 55 mutated to a cysteine and labeled with Alexa546) to the supported bilayers containing acceptor t-SNARE complexes. As seen in 7.12E, the level of bound synaptobervin-2 peptide increases as the concentration is increased, but increasing the concentration of complexin inhibits binding. These data were fit to a simple 1:1 binding isotherm and the results are plotted in Figure 7.12F. As seen in Figure 7.12F, at the highest concentration used $(2 \mu \mathrm{M})$ the effect of complexin has saturated and the affinity of synaptobrevin-2 for the reconstituted t-SNARE complex has been reduced by over a factor of 10 .

To determine whether this effect of complexin on the association of synaptobrevin- 2 and the binary plasma membrane complex correlates with physiological results, we tested the binding of synaptobrevin-2 using this approach in the presence of both super-clamping and non-clamping mutants of complexin-1. These mutants either slightly depress (the super-clamping) or dramatically increase (the non-clamping) spontaneous fusion events in cultured cortical neurons (Yang, et al., 2010). As seen in Figure 7.12F, the super-clamping mutant produces a response close to wild-type complexin, and may be just slightly more effective at reducing the affinity of 
synaptobrevin-2 to this complex. In contrast, the non-clamping mutant is much less effective at reducing the affinity of synaptobrevin-2 to this complex. These results correlate well with the physiological data and indicate that complexin acts by reducing the affinity of synaptobrevin-2 for the plasma membrane acceptor complex. 


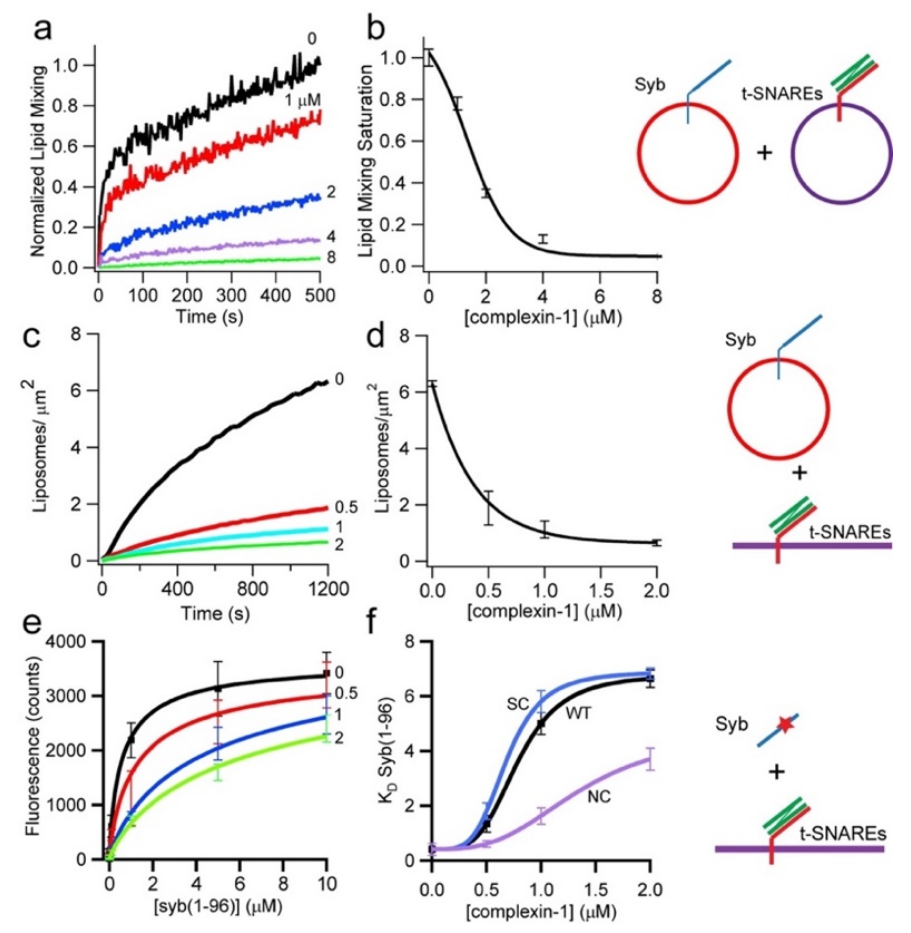

Figure 7.12: Complexin inhibits membrane fusion by reducing synaptobrevin-2 affinity for t-SNAREs. (a) Lipid mixing between synaptobrevin-2 and binary acceptor t-SNARE complex proteoliposomes (t-SNARE membrane composed of 70:30 POPC:POPS with a lipid:protein ratio of 400; v-SNARE membrane composed of 97:1.5:1.5 POPC:Rh-DOPE:NBD-DOPE, lipid:protein ratio of 400). Observed fluorescence as a function of time and presented as normalized signal intensity. After each fusion reaction, $0.1 \%$ Triton $\mathrm{X}-100$ was added to determine total fluorescence and normalize the data between different preparations. Traces correspond to lipid mixing in the absence of complexin (black) or in the presence of 1 (red), 2 (blue), 4 (violet), or $8 \mu \mathrm{M}$ complexin (green trace) as indicated. (b) Saturation of the lipid mixing taken at $500 \mathrm{sec}$ as a function of the concentration of added complexin. The inhibitory effect of complexin is concentration-dependent, and at $8 \mu \mathrm{M}$ complexin, complexin has reduced fusion by about 20 fold. Error bars are standard errors from 4 measurements. (c) Docking of synaptobrevin-2 proteoliposomes to SNARE acceptor complexes reconstituted into planar-supported bilayers (synaptobrevin-2 proteoliposomes composed of 99:1 POPC:Rh-DOPE) as a function of time. The docking was measured in the absence of complexin (black) and in the presence of $0.5 \mu \mathrm{M}$ (red), $1 \mu \mathrm{M}$ (cyan) and $2 \mu \mathrm{M}$ complexin (green) as indicated. (d) The number of bound synaptobrevin-2 proteoliposomes at $1200 \mathrm{sec}$ as a function of concentration of complexin. Values are averages from measurements on 3 bilayers, and errors are standard errors. (e) The binding of a fluorescently-labeled soluble synaptobrevin-2 (1-96) peptide following a 20 min incubation to a planar supported bilayer (70:30 POPC:POPS) containing the t-SNARE complex (SyxH3:dSNAP25) at a lipid:protein ratio of 3000. The binding of synaptobrevin-2 was measured without complexin (black), and at complexin concentrations of $0.5 \mu M$ (red), $1 \mu M$ (blue) and $2 \mu M$ (green). Values are averages of 3 measurements and errors are standard errors. (f) The Kd for synaptobrevin-2 binding calculated from the data in (e) is shown as a function of complexin concentration for wild type complexin (black trace) as well as the super-clamping (SC, blue trace) and non-clamping mutants (NC, purple trace). Error bars represent error in the fit. The mutations of SC are D27L, E34F, and R37A (Fasshauer, et al., 1997) and the mutations of NC are A30E, A31E, L41E, and A44E (Kümmel, et al., 2011). This figure was adapted from (Zdanowicz, et al., In Press). 


\subsection{Discussion}

Complexin-1 inhibits spontaneous release while at the same time synchronizing exocytosis when intracellular calcium levels rise. Some characteristics of the in vivo function of complexin have successfully been reconstituted (Lai, et al., 2014; Giraudo, et al., 2009; Malsam, et al., 2012; Lai, et al., 2016); however, biochemical studies of complexin and SNARE proteins have not revealed a clear mechanism by which complexin inhibits spontaneous fusion. The EPR and NMR spectroscopy, fluorescence anisotropy, and TIRF microscopy to characterize the interaction between complexin, SNAREs, and the lipid bilayer. We demonstrate that complexin not only binds to the assembled 4 helical coiled-coiled complex of syntaxin-1a:SNAP-25:synaptobrevin2, but also binds a binary (1:1) plasma membrane SNARE complex of syntaxin-1a:SNAP-25 with high affinity while simultaneously interacting with the lipid bilayer. These protein and membrane interactions of complexin are cooperative. The t-SNARE complex enhances the membrane affinity of complexin (Figure 7.9); and membrane associated SNAREs are also more likely to interact with complexin than their soluble counterparts. For example, complexin shows an affinity for d-SNAP-25 (Figure 7.11A, B and 7.10) that is not observed for SNAP-25 in solution (Figure 7.3). As shown previously (Kreutzberger, et al., 2016), this binary SNARE complex undergoes rapid fusion with synaptobrevin-2 vesicles. Using this system, we find that complexin inhibits calcium-independent fusion by inhibiting vesicle docking, and it does so by reducing the affinity of synaptobrevin-2 for the plasma membrane SNAREs. This reduction in synaptobrevin-2 affinity likely accounts for the decrease in spontaneous fusion events observed in the absence of calcium in vivo. 
As indicated above, previous reports have been controversial and inconsistent regarding the binding of complexin to the plasma membrane SNAREs. Part of the difficulty in characterizing this interaction may be due to the difficulty in producing plasma membrane SNARE complexes that are homogeneous and efficient at fusion. Syntaxin-1a and SNAP-25 efficiently form a 2:1 complex when reconstituted into membranes (Fasshauer, et al., 1997; Stein, et al., 2007). This off-pathway complex is slow to disassemble, and this disassembly accounts for the slow and inefficient fusion rates seen in some reconstituted systems. An artificially stabilized acceptor complex may be produced using the C-terminal end of synaptobrevin-2 (49-96), and this complex facilitates rapid and efficient fusion (Pobbati, et al., 2006). However, as seen in Figure 7.11B, the binding of complexin to this $\Delta \mathrm{N}$ complex is weak, as is the binding of complexin to a 2:1 (syntaxin-1a:SNAP-25) complex produced by a common reconstitution procedure using CHAPS. Binding to the fully assembled ternary cis-SNARE complex is stronger, but we observe the most efficient binding to the activated binary SNARE complex.

As seen in Figure 7.6 and 7.8, we observe efficient membrane binding at both the N- and Cterminal ends of complexin by EPR and NMR spectroscopy; moreover, both the N- and Cterminal interactions are dependent upon the curvature of the membrane, where the two interactions are independent of each other. These findings are generally consistent with recent reports. For example, curvature-dependent membrane binding at the $\mathrm{C}$-terminus has been reported for complexin from C. elegans (Snead, et al., 2014) and mammalian complexin (Gong, et al., 2016), and there is evidence that a fragment of complexin containing its $\mathrm{N}$-terminus participates in membrane binding (Lai, et al., 2016). In previous work, the strong curvature 
dependence of complexin binding was taken as evidence for an interaction of complexin with the vesicle membrane rather than an interaction with the plasma membrane (Snead, et al., 2014). However, whether complexin interacts with the vesicle membrane or the plasma membrane in vivo has not been established. From the dependence on curvature seen here (Figure 7.8), we estimate that either end of complexin will exhibit a roughly 10 -fold higher affinity towards the synaptic vesicle membrane; however, given the strong plasma membrane SNARE interactions of complexin, the $\mathrm{N}$ - and C-terminal domains may associate with the plasma membrane simply because of their proximity to this membrane. Of course, one can imagine that complexin binds to the acceptor plasma membrane SNARE complex and the vesicle membrane when the two are in close proximity, as they will be when synaptobrevin-2 inserts into the plasma membrane SNARE complex. Regardless of the sequence of events, both membrane and plasma membrane SNARE interactions of complexin will contribute to its overall association, and the removal of any region that contributes to either plasma membrane SNARE or membrane binding may reduce the effectiveness of complexin. This cooperativity likely explains why removal of the complexin Cterminus modulates complexin activity (Wragg, et al., 2013).

A number of model have been proposed to account for the inhibitory action of complexin on membrane fusion. In one, complexin binds to the SNAREs to yield a state that is "clamped" and not yet able to fully assemble and drive fusion (Tang, et al., 2006). In this state, the calcium sensor synaptotagmin-1 triggers synchronous release by binding the SNAREs and displacing complexin. However, work that is more recent shows that complexin and synaptotagmin may both simultaneously bind SNAREs, leading to an alternate model where synaptotagmin binding 
rearranges the conformation of complexin when bound to the SNAREs (Park, et al., 2015).

Complexin-1 has also been proposed to arrest SNARE assembly by crosslinking partially assembled SNAREs through its accessory helix (Kümmel, et al., 2011). This model has been the subject of some debate and whether the accessory helix interacts across SNAREs has not been resolved (Trimbuch, et al., 2014; Krishnakumar, et al., 2015). There is also a report that complexin can exist in two configurations, one where it interacts exclusively with the ternary post-fusion complex and a second where complexin bridges the ternary complex with a binary complex (Choi, et al., 2016). How such an interaction might regulate SNARE assembly is not clear. The data shown here (Figure 7.6) indicate that both the N- and C-termini of complexin simultaneously interact with membranes; as a result, interactions of the accessory helix across SNAREs may not be structurally feasible. There has also been a proposal that electrostatic repulsion between vesicle and target membranes by the complexin accessory helix accounts for the inhibitory activity of complexin (Trimbuch, et al., 2014). However, such a model requires that the repulsive interactions be sufficiently large to inhibit SNARE assembly, which requires that the physical dimensions over which the repulsive interactions act be less than the Debye length, or less than $1 \mathrm{~nm}$ under physiological buffer conditions. All these models are built on the premise of a rapid and precisely timed recruitment of complexin to the ternary SNARE complex where synaptobrevin-2 is in various demonstrate that complexin will associate with the binary target SNARE complex in the absence of vesicle SNAREs and potentially before the initiation of the vesicle and plasma membrane SNARE assembly, providing a plausible starting point for the inhibitory action of complexin. 
In summary, the data shown here clearly demonstrate that complexin inhibits the docking of vesicle SNARE vesicles and weakens the affinity of synaptobrevin-2 for an acceptor SNARE complex, and it does so by simultaneous membrane and SNARE binding. These observations support a model, similar to one previously proposed (Yoon, et al., 2008), in which complexin acts to lower the frequency of spontaneous fusion events in neuronal exocytosis by binding to the acceptor SNARE complex and delaying assembly of the ternary SNARE complex. Although many details regarding the state of the SNAREs and the role of synaptotagmin are still unknown, it is likely that syntaxin-1a and SNAP-25 are in at some stage a 1:1 complex with the regulatory protein Munc18 and perhaps Munc13 which regulate their assembly and allow synaptotagmin to trigger calcium mediated fusion. In the following chapter, these components will be examined alongside of complexin to better understand how fusion is coordinated to respond to calcium. 


\section{Chapter 8: Reconstitution of calcium dependent fusion}

Kreutzberger, A.J.B., V. Kiessling, B. Liang, P. Seelheim, S. Jakhanwal, R. Jahn, J.D. Castle, and L.K. Tamm (submitted).

The design of using dense core vesicles labeled with a fluorescent NPY in the planar supported bilayer fusion assay came from Volker Kiessling and David Castle. The purification of proteins was done by Binyong Liang, and the method for purifying the C1C2Mun construct of Munc13 came from Shrutee Jakhanwal in the lab of Reinhard Jahn. Plasmids for knockdowns and recoveries were designed and made by Patrick Seelheim.

\subsection{Summary}

Regulated exocytosis is a process by which neurotransmitters, hormones, and secretory proteins are released from the cell in response to elevated levels of calcium. In cells, secretory vesicles are targeted to the plasma membrane, where they dock, undergo priming, and then fuse with the plasma membrane in response to calcium. The specific roles of essential proteins and how calcium regulates progression through these sequential steps currently are incompletely resolved. Here we have used purified neuroendocrine dense core vesicles and artificial membranes to reconstruct in vitro the serial events that mimic SNARE-dependent membrane docking and fusion during exocytosis. Calcium recruits these vesicles to the target membrane aided by the protein CAPS while synaptotagmin catalyzes calcium-dependent fusion; both these processes are dependent on PI4,5 $\mathrm{P}_{2}$. The soluble proteins Munc18 and complexin-1 are necessary to arrest 
vesicles in a docked state in the absence of calcium, while CAPS and/or Munc13 are involved in priming the system for an efficient fusion reaction.

\subsection{Introduction}

Regulated exocytosis is used by cells to export stored secretory products - proteins, neurotransmitters, hormones and small molecules - in response to calcium-mediated signaling (Martens \& McMahon, 2008). In neurons and neuroendocrine cells, the final step of exocytosis is membrane fusion, which is catalyzed by the SNARE proteins syntaxin-1 and SNAP-25 in the plasma membrane and synaptobrevin-2/VAMP-2 in the secretory vesicle membrane (Martens \& McMahon, 2008; Jahn \& Fasshauer, 2012; Südhof, 2013). While SNARE proteins are sufficient to catalyze membrane fusion (Weber, et al., 1998), several other proteins are involved in regulating the assembly of SNARE complexes and in coupling the calcium signal to the fusion process (Jahn \& Fasshauer, 2012; Südhof, 2013). These include synaptotagmin, complexin, Munc18, Munc13, and CAPS (calcium active protein for secretion) (Jahn \& Fasshauer, 2012; Südhof, 2013; James \& Martin, 2013). Numerous studies have identified prospective roles for these proteins in the vesicle docking, priming, and triggering steps that contribute to exocytosis in situ (Ann, et al., 1997; Gerber, et al., 2008; Nguyen Truong, et al., 2014; Tang, et al., 2006; Borisovska, et al., 2005; Kabachinski, et al., 2016) while others have analyzed how their interactions affect reconstituted SNARE-mediated fusion in vitro (Diao, et al., 2012; Lai, et al., 2014; Kiessling, et al., 2013; Yoon, et al., 2008; Ma, et al., 2013). However, significant gaps remain in a precise molecular understanding of how and in what order the specific interactions among these proteins relate to the steps of the exocytotic process and interface with calcium's actions in triggering release. Most importantly, while several in vitro studies have reported an 
enhancement of fusion by calcium, complexin, and synaptotagmin under specialized conditions (Diao, et al., 2012; Kyoung, et al., 2011), it has not been possible so far to reconstitute a fusionarrested docked state in vitro in which a robust fusion response is triggered by calcium under conditions containing all known in situ requirements for exocytosis.

\subsection{Results}

DCV fusion was SNARE-dependent in all conditions tested in the planar supported bilayer assay (Figure 8.1 and Table 8.1). Lipid mixing in the ensemble fusion assay was accelerated by calcium, Figure 8.2 and Table 8.2, consistent with results for other purified secretory vesicles preparations (Park, et al., 2012; Park, et al., 2015). The amount of docking as well as the rate and probability of fusion of DCVs to supported bilayers were enhanced by calcium (Figure 8.3, Table 8.3, and Table 8.4). Calcium-dependent docking did not require SNARE proteins but fusion only occurred when both syntaxin-1a and SNAP25 were present (Figure 8.1). Calciumdependent docking and fusion in the presence of calcium were enhanced by $\mathrm{PI}, 5 \mathrm{P}_{2}$ and reduced in the absence of charged lipids (Figure 8.4, Table 8.5, and Table 8.6) which suggests that $\mathrm{PI} 4,5 \mathrm{P}_{2}$ could control the calcium sensitivity of fusion to have the high affinity observe in situ. This is in agreement with the necessity of PI4,5 $\mathrm{P}_{2}$ that has been observed in cells (Martin, 2012). 


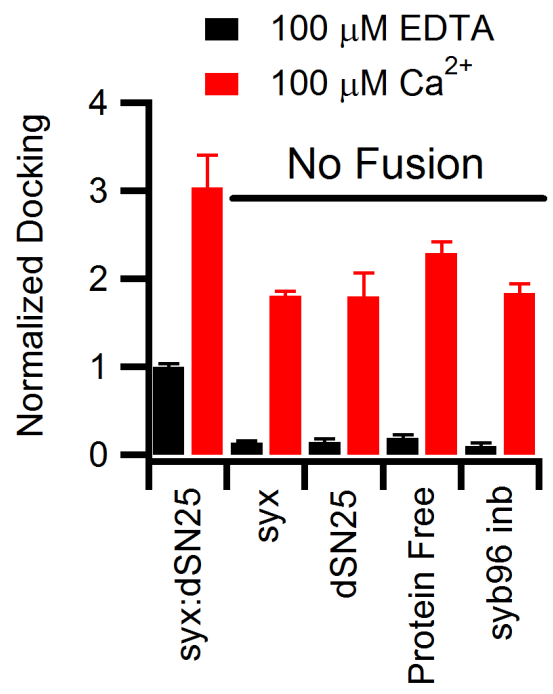

Figure 8.1: Docking of DCVs to planar supported bilayers that differ in t-SNARE content in the absence (black) and present (red) of $\mathrm{Ca}^{2+}$. Conditions are in the presence of syntaxin-1a (183288):d-SNAP25, syntaxin-1a (183-288) only, d-SNAP25 only, no proteins, or with syntaxin-1a(183288):d-SNAP25 in the presence of $2 \mu M$ synaptobrevin-2 (1-96) inhibitor peptide. Fusion was observed only in the presence of syntaxin-1a(183-288):d-SNAP25. Table 8.1 shows a summary of all observed events under these conditions. This figure was adapted from (Kreutzberger, et al., Submitted).

\begin{tabular}{cccccc}
\hline Condition & $\begin{array}{c}\text { Number of } \\
\text { Bilayers }\end{array}$ & $\begin{array}{c}\text { Normalized } \\
\text { Docking }\end{array}$ & $\begin{array}{c}\text { Percent } \\
\text { Fusion }\end{array}$ & $\begin{array}{c}\text { Total Number } \\
\text { of Docking } \\
\text { Events }\end{array}$ & $\begin{array}{c}\text { Total Number } \\
\text { of Fusion } \\
\text { Events }\end{array}$ \\
\hline syx(183-288):dSN25 EDTA & 14 & $1.0 \pm 0.1$ & $41.0 \pm 0.9$ & 823 & 340 \\
syx(183-288):dSN25 Ca ${ }^{2+}$ & 7 & $3.0 \pm 0.5$ & $64.4 \pm 3.0$ & 1753 & 1184 \\
syx(183-288) EDTA & 5 & $0.14 \pm 0.02$ & -- & 38 & 4 \\
syx(183-288) Ca ${ }^{2+}$ & 5 & $1.81 \pm 0.09$ & -- & 468 & 7 \\
dSN25 EDTA & 5 & $0.15 \pm 0.04$ & -- & 27 & 1 \\
dSN25 Ca ${ }^{2+}$ & 5 & $1.8 \pm 0.3$ & -- & 351 & 6 \\
Protein Free EDTA & 5 & $0.19 \pm 0.04$ & -- & 41 & 4 \\
Protein Free Ca ${ }^{2+}$ & 5 & $2.3 \pm 0.2$ & -- & 434 & 7 \\
syx(183-288):dSN25 syx(1-96) EDTA & 5 & $0.10 \pm 0.04$ & -- & 19 & 2 \\
syx(183-288):dSN25 syx(1-96) Ca ${ }^{2+}$ & 5 & $1.8 \pm 0.1$ & -- & 368 & 3 \\
\hline
\end{tabular}

Table 8.1: Event statistics for DCV docking and fusion to planar supported bilayers with syntaxin1a (183-288):d-SNAP25 (lipid:protein 3000), syntantaxin-1a(183-288) (lipid to protein of 3000), dSNAP25 (lipid:protein of 3000), no protein, or syntaxin-1a:d-SNAP25 (lipid:protein of 3000) with 2 $\mu \mathrm{M}$ synaptobrevin-2 (1-96) inhibitor peptide. All conditions were measured in the presence of 100 $\mu \mathrm{M}$ EDTA or $100 \mu \mathrm{M} \mathrm{Ca}{ }^{2+}$. This figure was adapted from (Kreutzberger, et al., Submitted). 
A)

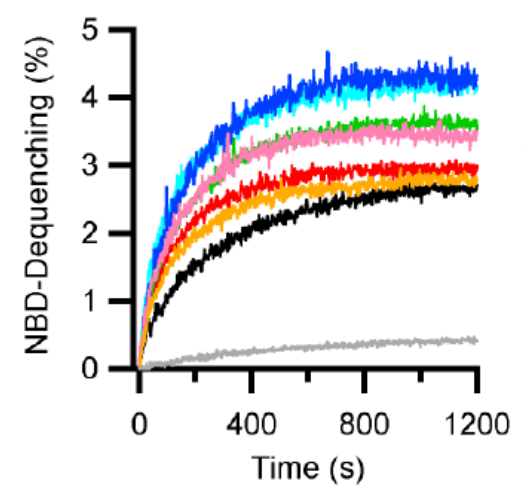

B)

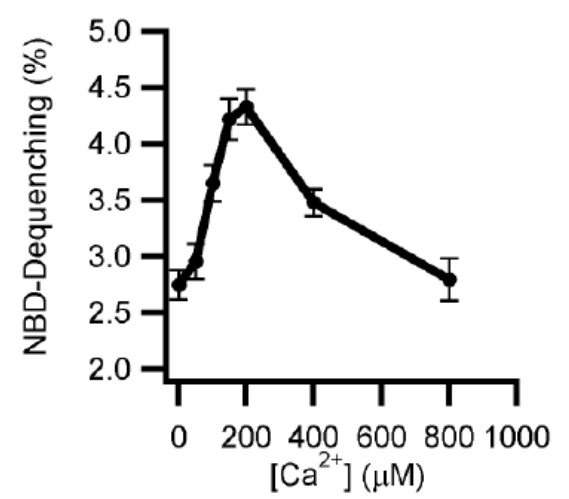

Figure 8.2: Ensemble lipid mixing of DCVs with reconstituted proteoliposomes containing syntaxin-1a (183-288):d-SNAP25 at a lipid to protein ratio of 500 with a lipid composition of bPC:bPE:bPS:Chol:PI:PI4,5P 2 :Rh-DOPE:NBD-DOPE (23.5:23.5:15:30:4:1:1.5:1.5) at increasing $\left[\mathrm{Ca}^{2+}\right]$. (A) Shows averaged lipid mixing traces (4 traces) at increasing $\left[\mathrm{Ca}^{2+}\right]$ or in the presence of 2 $\mu \mathrm{M}$ synaptobrevin-2 (1-96) inhibitor. (B) Saturation of lipid mixing at increasing $\left[\mathrm{Ca}^{2+}\right]$; error bars reflect standard error of repeated lipid mixing traces. Fitting the initial increase and saturation of the $\mathrm{Ca}^{2+}$ response results in a $\mathrm{K}_{1 / 2}\left[\mathrm{Ca}^{2+}\right]$ of $97 \pm 18 \mu \mathrm{M}$. Summary of lipid mixing data is shown in Table 8.2. This figure was adapted from (Kreutzberger, et al., Submitted).

\begin{tabular}{cccc}
\hline$\left[\mathrm{Ca}^{2+}\right]$ & Saturation & $\mathbf{k}_{\mathbf{1}}\left(\mathbf{s}^{-1}\right)$ & $\mathbf{k}_{\mathbf{2}}\left(\mathbf{s}^{-1}\right)$ \\
\hline 0 & $2.8 \pm 0.1$ & $0.0028 \pm 0.0001$ & $0.022 \pm 0.003$ \\
50 & $3.0 \pm 0.2$ & $0.0048 \pm 0.0001$ & $0.024 \pm 0.003$ \\
100 & $3.7 \pm 0.2$ & $0.0044 \pm 0.0001$ & $0.03 \pm 0.003$ \\
150 & $4.2 \pm 0.2$ & $0.0041 \pm 0.0001$ & $0.026 \pm 0.002$ \\
200 & $4.3 \pm 0.2$ & $0.0047 \pm 0.0001$ & $0.037 \pm 0.007$ \\
400 & $3.5 \pm 0.1$ & $0.0056 \pm 0.0001$ & $0.07 \pm 0.01$ \\
800 & $2.8 \pm 0.2$ & $0.0040 \pm 0.0001$ & $0.025 \pm 0.002$ \\
\hline
\end{tabular}

Table 8.2: Fits of ensemble lipid mixing data shown in Figure 8.2. Averages of 4 traces were taken for each condition. All curves were fit with a two-component model being $y=A_{1}\left(1-e^{-k 1^{*} t}\right)+A_{2}\left(1-e^{-}\right.$ $\left.{ }^{k 2 *}\right)$. This figure was adapted from (Kreutzberger, et al., Submitted). 
A)

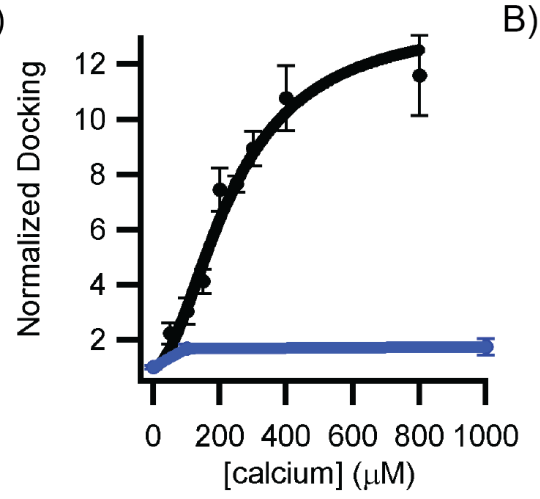

B)

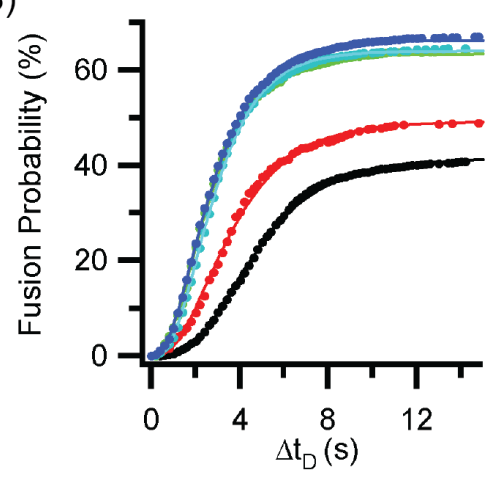

C)

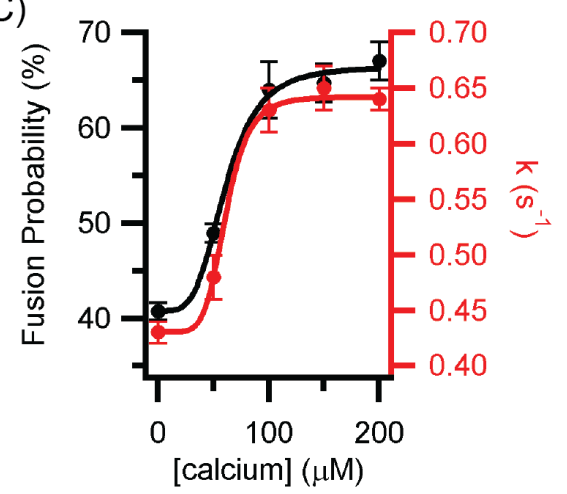

Figure 8.3: (A) Docking as a function of $\left[\mathrm{Ca}^{2+}\right]$ (black) or $\left[\mathrm{Mg}^{2+}\right]$ (blue) in the single vesicle planar supported bilayer assay with a $\mathrm{K}_{1 / 2}\left[\mathrm{Ca}^{2+}\right]$ of $236 \pm 46 \mu \mathrm{M}$. Table 8.3 contains a summary of total number of docking and fusion events. (B) Delay time between docking and fusion $\left(\Delta t_{D}\right)$ at different $\left[\mathrm{Ca}^{2+}\right]$ shown as cumulative distribution functions of single $\mathrm{DCV}$ fusion events normalized to the fusion probability. Summary of data is Table 8.3. The kinetics were fit with a parallel reaction model $N(t)=N\left(1-e^{-k t}\right)^{m}$ where $N$ is the fusion probability, $k$ is the rate, and $m$ is the number of parallel reactions occurring (Domanska, et al., 2009). Summary of fit values are shown in Table 8.4. The curves are for $100 \mu \mathrm{M}$ EDTA (black), $50 \mu \mathrm{M} \mathrm{Ca}^{2+}$ (red), $100 \mu \mathrm{M} \mathrm{Ca}^{2+}$ (cyan), $150 \mu \mathrm{M} \mathrm{Ca}^{2+}$ (blue), and $200 \mu \mathrm{M} \mathrm{Ca}^{2+}$ (green). (C) The fusion probabilities (black) and rate constants (red) for parallel reactions are shown as functions of $\left[\mathrm{Ca}^{2+}\right]$ with $\mathrm{K}_{1 / 2}\left[\mathrm{Ca}^{2+}\right]$ of $60 \pm 8 \mu \mathrm{M}$ and $61 \pm 8 \mu \mathrm{M}$, respectively. (A-C) All membranes contained syntaxin-1a(183-288):d-SNAP25 at lipid:protein of

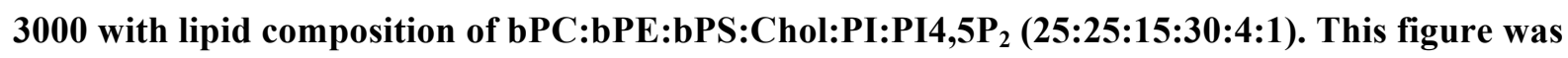
adapted from (Kreutzberger, et al., Submitted). 


\begin{tabular}{cccccc}
\hline Condition & $\begin{array}{c}\text { Number of } \\
\text { Bilayers }\end{array}$ & $\begin{array}{c}\text { Normalized } \\
\text { Docking }\end{array}$ & $\begin{array}{c}\text { Percent } \\
\text { Fusion }\end{array}$ & $\begin{array}{c}\text { Total Number } \\
\text { of Docking } \\
\text { Events }\end{array}$ & $\begin{array}{c}\text { Total Number } \\
\text { of Fusion } \\
\text { Events }\end{array}$ \\
\hline $\mathbf{1 0 0} \boldsymbol{\mu} \mathbf{M ~ E D T A}$ & 14 & $1.0 \pm 0.1$ & $40.1 \pm 0.9$ & 823 & 340 \\
$\mathbf{5 0} \boldsymbol{\mu} \mathrm{M} \mathrm{Ca}^{2+}$ & 5 & $2.2 \pm 0.4$ & $49 \pm 1$ & 817 & 394 \\
$\mathbf{1 0 0} \boldsymbol{\mu} \mathrm{M} \mathrm{Ca}^{2+}$ & 7 & $3.0 \pm 0.5$ & $64 \pm 3$ & 1753 & 1184 \\
$\mathbf{1 5 0} \boldsymbol{\mu} \mathrm{M} \mathrm{Ca}^{2+}$ & 5 & $4.1 \pm 0.4$ & $64.7 \pm 0.5$ & 1840 & 1188 \\
$\mathbf{2 0 0} \boldsymbol{\mu} \mathrm{M} \mathrm{Ca}^{2+}$ & 5 & $7.5 \pm 0.8$ & $67 \pm 1$ & 3233 & 2169 \\
$\mathbf{2 5 0} \boldsymbol{\mu} \mathbf{M ~ C a}^{2+}$ & 5 & $7.7 \pm 0.3$ & $60 \pm 1$ & 1150 & 693 \\
$\mathbf{3 0 0} \boldsymbol{\mu} \mathbf{M ~ C a}^{2+}$ & 5 & $9.0 \pm 0.6$ & $59 \pm 1$ & 1343 & 797 \\
$\mathbf{4 0 0} \boldsymbol{\mu} \mathbf{M ~ C a}^{2+}$ & 5 & $10.8 \pm 1.1$ & $54 \pm 2$ & 3264 & 1766 \\
$\mathbf{8 0 0} \boldsymbol{\mu} \mathbf{M ~ C a}^{2+}$ & 5 & $11.6 \pm 1.4$ & $50 \pm 1$ & 3965 & 1968 \\
$\mathbf{1 0 0} \boldsymbol{\mu} \mathbf{M ~ M g}^{2+}$ & 5 & $1.7 \pm 0.2$ & $45 \pm 2$ & 320 & 145 \\
$\mathbf{1 0 0 0} \boldsymbol{\mu} \mathbf{M ~ M g}^{2+}$ & 5 & $1.8 \pm 0.3$ & $50 \pm 2$ & 306 & 155 \\
\hline
\end{tabular}

Table 8.3: Event statistics for DCV docking and fusion to planar supported bilayers with syntaxin1a (183-288):d-SNAP25 (lipid:protein of 3000) with increasing concentration of divalent metals $\left(\mathrm{Ca}^{2+}\right.$ and $\left.\mathrm{Mg}^{2+}\right)$. This figure was adapted from (Kreutzberger, et al., Submitted).

\begin{tabular}{|c|c|c|c|}
\hline Condition & $\begin{array}{l}\text { Number of } \\
\text { Bilayers }\end{array}$ & $k\left(s^{-1}\right)$ & $m$ \\
\hline $100 \mu \mathrm{M}$ EDTA & 14 & $0.44 \pm 0.01$ & $4.8 \pm 0.2$ \\
\hline $50 \mu \mathrm{M} \mathrm{Ca}^{2+}$ & 5 & $0.48 \pm 0.02$ & $3.3 \pm 0.2$ \\
\hline $100 \mu \mathrm{M} \mathrm{Ca}^{2+}$ & 7 & $0.63 \pm 0.02$ & $3.1 \pm 0.1$ \\
\hline $150 \mu \mathrm{M} \mathrm{Ca}^{2+}$ & 5 & $0.65 \pm 0.02$ & $3.7 \pm 0.1$ \\
\hline $200 \mu \mathrm{M} \mathrm{Ca}^{2+}$ & 5 & $0.61 \pm 0.01$ & $3.0 \pm 0.1$ \\
\hline $250 \mu \mathrm{M} \mathrm{Ca}^{2+}$ & 5 & $0.54 \pm 0.02$ & $3.0 \pm 0.1$ \\
\hline $300 \mu \mathrm{M} \mathrm{Ca}^{2+}$ & 5 & $0.53 \pm 0.02$ & $3.2 \pm 0.1$ \\
\hline $400 \mu \mathrm{M} \mathrm{Ca}^{2+}$ & 5 & $0.49 \pm 0.01$ & $5.3 \pm 0.2$ \\
\hline $800 \mu \mathrm{M} \mathrm{Ca}{ }^{2+}$ & 5 & $0.43 \pm 0.01$ & $4.4 \pm 0.1$ \\
\hline $100 \mu \mathrm{M} \mathrm{Mg}^{2+}$ & 5 & $0.39 \pm 0.04$ & $3.4 \pm 0.4$ \\
\hline $1000 \mu \mathrm{M} \mathrm{Mg}^{2+}$ & 5 & $0.35 \pm 0.03$ & $2.6 \pm 0.2$ \\
\hline
\end{tabular}

Table 8.4: Fit values of a parallel reaction model $\left(N(t)=N\left(1-e^{-k t}\right)^{m}\right.$ where $N$ is the fusion probability, $k$ is the rate, and $m$ is the number of parallel reactions occurring) for the cumulative distribution function of delay times between docking and fusion for single particle DCV events with different concentrations of divalent metals. Experimental traces are shown in Figure 8.2B. Planar bilayers contained syntaxin-1a(183-288):d-SNAP25 (lipid:protein of 3000 and lipid composition 25:25:15:30:4:1 bPE:bPE:bPS:Chol:PI:PI4,5P 2 ). This figure was adapted from (Kreutzberger, et al., Submitted). 

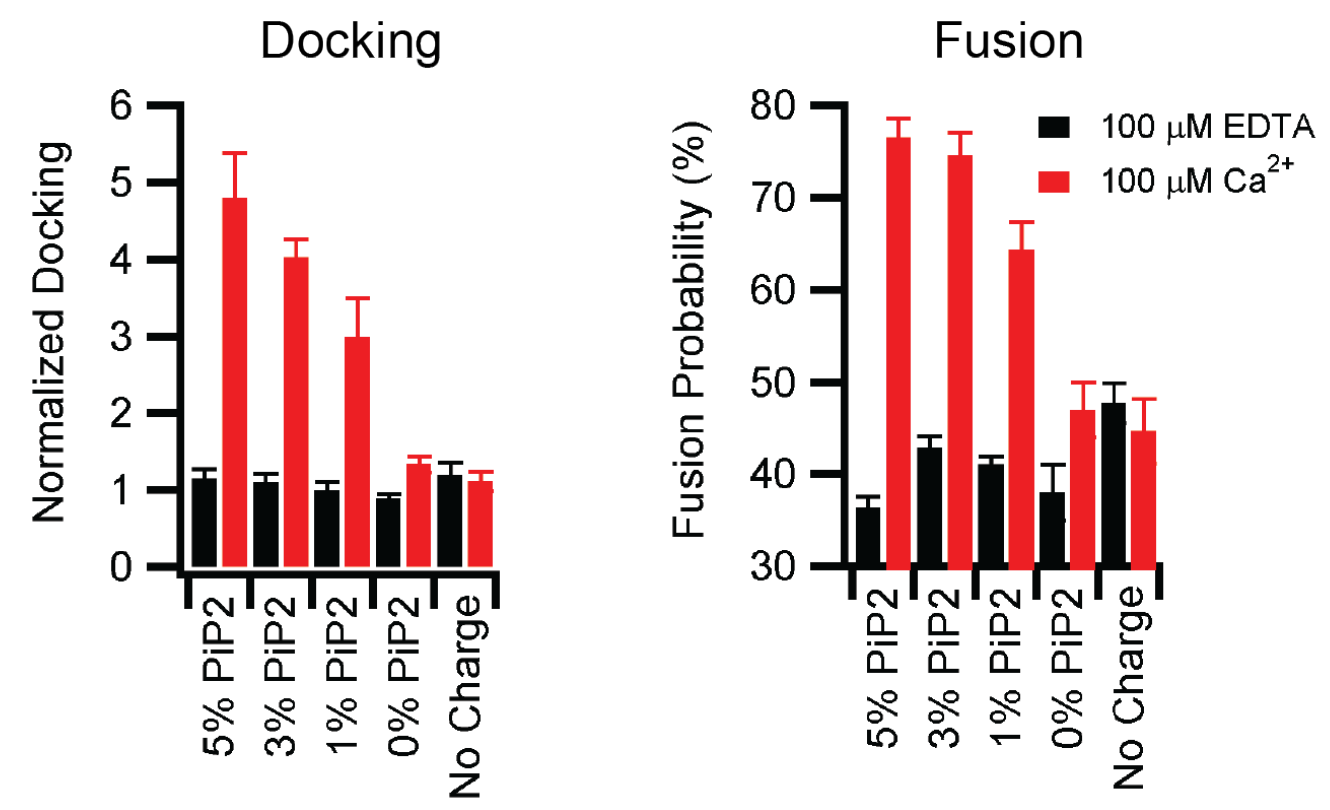

Figure 8.4: The effect on docking and fusion of DCVs with $\mathrm{PI} 4,5 \mathrm{P}_{2}$ is removed from planar bilayers containing bPC:bPE:bPS:Chol:(PI+PI4,5P $)(25: 25: 15: 30: 5)$ or in the absence of charged lipids (bPE:bPE:Chol, 35:35:30). Summaries of docking and fusion events and fitting results of fusion kinetics are shown in Tables 8.5 and 8.6, respectively. This figure was adapted from (Kreutzberger, et al., Submitted). 


\begin{tabular}{|c|c|c|c|c|c|}
\hline Condition & $\begin{array}{l}\text { Number of } \\
\text { Bilayers }\end{array}$ & $\begin{array}{l}\text { Normalized } \\
\text { Docking }\end{array}$ & $\begin{array}{l}\text { Percent } \\
\text { Fusion }\end{array}$ & $\begin{array}{l}\text { Total Number } \\
\text { of Docking } \\
\text { Events }\end{array}$ & $\begin{array}{l}\text { Total Number of } \\
\text { Fusion Events }\end{array}$ \\
\hline $5 \% \mathrm{Pl} 4,5 \mathrm{P}_{2}$ - EDTA & 5 & $1.2 \pm 0.1$ & $36 \pm 1$ & 294 & 108 \\
\hline $5 \% \mathrm{Pl} 4,5 \mathrm{P}_{2} \mathrm{Ca}^{2+}$ & 5 & $4.8 \pm 0.6$ & $76 \pm 2$ & 1223 & 932 \\
\hline $3 \% \mathrm{Pl}_{1}, 5 \mathrm{P}_{2}$ - EDTA & 5 & $1.1 \pm 0.1$ & $42 \pm 1$ & 280 & 120 \\
\hline $3 \% \mathrm{P} 44,5 \mathrm{P}_{2} \mathrm{Ca}^{2+}$ & 4 & $4.0 \pm 0.2$ & $75 \pm 2$ & 822 & 611 \\
\hline $1 \% \mathrm{P} 4,5 \mathrm{P}_{2}$ - EDTA & 14 & $1.0 \pm 0.1$ & $41 \pm 1$ & 823 & 340 \\
\hline $1 \% \mathrm{Pl} 4,5 \mathrm{P}_{2} \mathrm{Ca}^{2+}$ & 7 & $3.0 \pm 0.5$ & $64 \pm 3$ & 1753 & 1184 \\
\hline $0 \% \mathrm{Pl} 4,5 \mathrm{P}_{2}$ - EDTA & 5 & $0.9 \pm 0.1$ & $38 \pm 3$ & 211 & 82 \\
\hline $0 \% \mathrm{P} \mid 4,5 \mathrm{P}_{2} \mathrm{Ca}^{2+}$ & 6 & $1.3 \pm 0.1$ & $47 \pm 3$ & 386 & 183 \\
\hline No Charge - EDTA & 5 & $1.2 \pm 0.2$ & $47 \pm 2$ & 274 & 133 \\
\hline No Charge $-\mathrm{Ca}^{2+}$ & 5 & $1.1 \pm 0.1$ & $45 \pm 4$ & 259 & 117 \\
\hline
\end{tabular}

Table 8.5: Event statistics for docking and fusion of DCVs to planar supported bilayers with different concentrations of PI4,5 $\mathrm{P}_{2}$ with membrane composition of bPC:bPE:bPS:Chol: $\left(\mathrm{PI}+\mathrm{PI} 4,5 \mathrm{P}_{2}\right)(25: 25: 15: 30: 5)$ and in membranes without charge (bPC:bPE:Chol 35:35:30) in the presence of $100 \mu \mathrm{M}$ EDTA or $100 \mu \mathrm{M} \mathrm{Ca}{ }^{2+}$. This figure was adapted from (Kreutzberger, et al., Submitted).

\begin{tabular}{ccc}
\hline Condition & $\mathbf{k}\left(\mathbf{s}^{-\mathbf{1}}\right)$ & $\mathbf{m}$ \\
\hline $5 \% \mathrm{Pl}, 5 \mathrm{P}_{2}-\mathrm{EDTA}$ & $0.35 \pm 0.05$ & $3.5 \pm 0.5$ \\
$5 \% \mathrm{Pl} 4,5 \mathrm{P}_{2}-\mathrm{Ca}^{2+}$ & $0.63 \pm 0.05$ & $2.4 \pm 0.2$ \\
$3 \% \mathrm{Pl} 4,5 \mathrm{P}_{2}-\mathrm{EDTA}$ & $0.36 \pm 0.04$ & $2.8 \pm 0.3$ \\
$3 \% \mathrm{Pl} 4,5 \mathrm{P}_{2}-\mathrm{Ca}^{2+}$ & $0.61 \pm 0.05$ & $2.4 \pm 0.1$ \\
$1 \% \mathrm{Pl} 4,5 \mathrm{P}_{2}-\mathrm{EDTA}$ & $0.44 \pm 0.10$ & $4.8 \pm 0.2$ \\
$1 \% \mathrm{Pl} 4,5 \mathrm{P}_{2}-\mathrm{Ca}^{2+}$ & $0.63 \pm 0.02$ & $3.1 \pm 0.1$ \\
$0 \% \mathrm{Pl} 4,5 \mathrm{P}_{2}-\mathrm{EDTA}^{2}$ & $0.42 \pm 0.05$ & $4.9 \pm 0.8$ \\
$0 \% \mathrm{Pl} 4,5 \mathrm{P}_{2}-\mathrm{Ca}^{2+}$ & $0.35 \pm 0.03$ & $3.3 \pm 0.3$ \\
No Charge $-\mathrm{EDTA}^{2}$ & $0.42 \pm 0.03$ & $4.4 \pm 0.5$ \\
No Charge $-\mathrm{Ca}^{2+}$ & $0.43 \pm 0.04$ & $4.0 \pm 0.5$ \\
\hline
\end{tabular}

Table 8.6: Event statistics for docking and fusion of DCVs to planar supported bilayers with different concentrations of PI4,5P $\mathrm{P}_{2}$ with membrane composition of bPC:bPE:bPS:

Chol:(PI+PI4,5P $)(25: 25: 15: 30: 5)$ and in membranes without charge (bPC:bPE:Chol 35:35:30) in the presence of $100 \mu \mathrm{M}$ EDTA or $100 \mu \mathrm{M} \mathrm{Ca}^{2+}$. The fit is of a parallel reaction model being $\mathrm{N}(\mathrm{t})=$ $\mathrm{N}\left(1-\mathrm{e}^{-\mathrm{kt}}\right)^{\mathrm{m}}$ where $\mathrm{N}$ is the fusion probability, $\mathrm{k}$ is the rate, and $\mathrm{m}$ is the number of parallel reactions occurring. This figure was adapted from (Kreutzberger, et al., Submitted). 
Both synaptotagmin, a resident integral protein of synaptic vesicles and DCVs, and CAPS, a soluble but also vesicle associated protein (Figure 3.6), have been implicated in regulation of exocytosis by calcium (James \& Martin, 2013). Numerous studies have identified syaptotagmin as a calcium sensor that triggers fusion (Südhof, 2013; Brose, et al., 1992). Notably, synaptotagmin has been shown to accelerate fusion by interacting with PI4,5 $\mathrm{P}_{2}$ (Park, et al., 2012; Park, et al., 2015; Wang, et al., 2011). Some of these effects could be due to increased crosslinking of vesicles by synaptotagmin although single particle fusion assays have indicated that synaptotagmin may have little effect on docking in the presence of SNARE proteins (Kiessling, et al., 2013). CAPS, which was originally identified as a fusion trigger (Hay \& Martin, 1992), includes a PI4,5 $\mathrm{P}_{2}$-binding PH domain, a calcium binding $\mathrm{C} 2$ domain, and a syntaxin-1a interacting MUN domain (James \& Martin, 2013). While it has received less attention than synaptotagmin, and as been questioned previously as a prospective fusion trigger (Grishanin, et al., 2004), recent studies have shown convincing roles of CAPS in the more proximal steps of docking and priming of DCVs for fusion(James \& Martin, 2013; Nguyen Truong, et al., 2014; Kabachinski, et al., 2016). The abilities to examine both docking and fusion of DCVs to supported bilayers and to manipulate the levels and functions of DCV-associated synaptotagmin and CAPS have provided us a unique opportunity to examine the roles of these proteins comparatively. We have utilized two approaches toward this goal. The first is to add function-blocking antibodies to the in vitro assay. The second is to use shRNA-mediated protein depletion in PC12 cells prior to purification of DCVs. In the case of synaptotagmin, the antisynaptotagmin antibodies, which bind to the $\mathrm{C} 2$ domains, had no effect on the extent of calciumenhanced docking (Figure 8.6A). As well, shRNA-mediated knockdown of synaptotagmin-1 and -9 (the DCV-associated synaptotagmins that control exocytosis (Lynch \& Martin, 2007) to $>85 \%$ 
for both isoforms (Figure 8.5) did not affect the extent of docking (Figure 8.6A). However, both treatments profoundly inhibited calcium-accelerated fusion (Figure 8.6B and Figure 8.7A).

Notably, the effect on fusion was rescued by expressing RNAi-resistant synaptotagmin-1 during knockdown (Figure 8.6 A, B).

A)

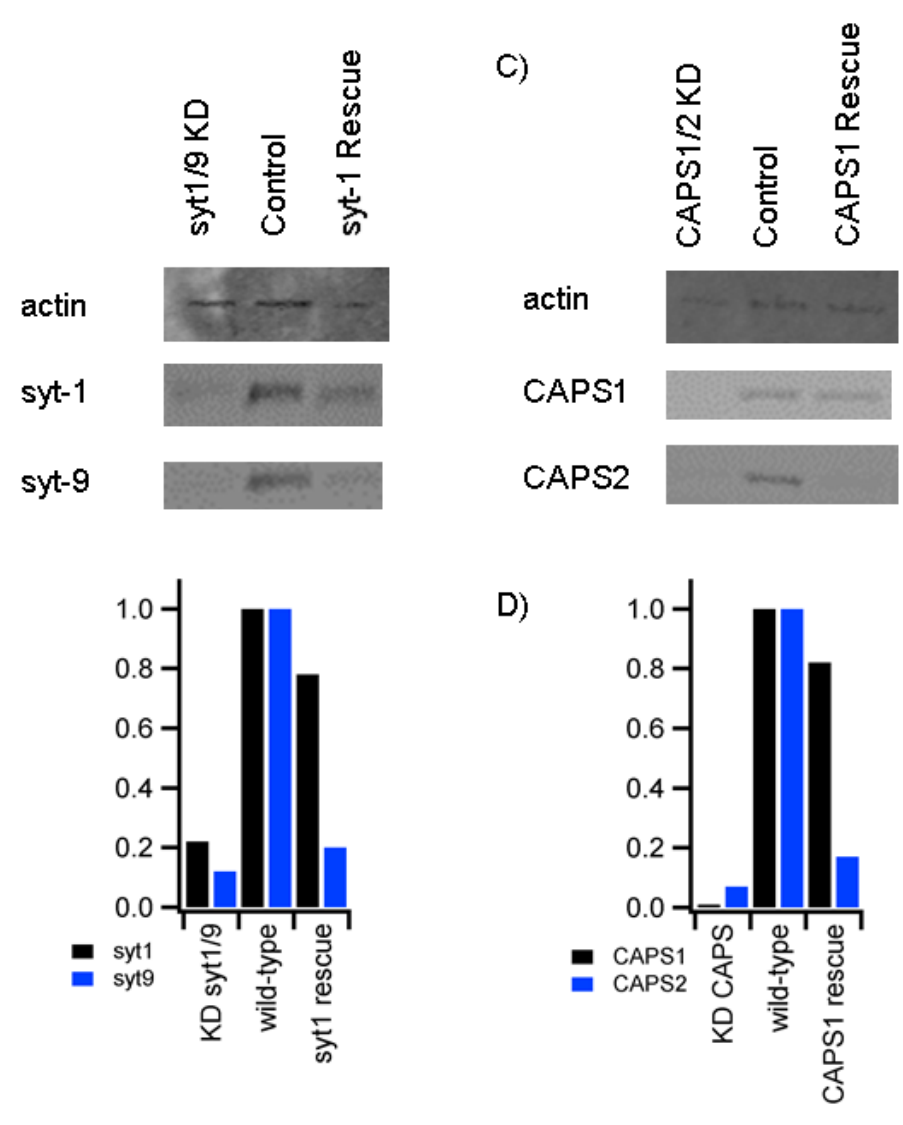

Figure 8.5: Western blots of shRNA-mediated knockdowns for (A) syt1/9 or (B) CAPS1/2. Blots were of total cell lysates with approximately equal amounts of protein loaded onto each gel which was then normalized to total amount of actin per lysate to account for slight differences in total number of cells (B and D). (B) Syt1 was knocked down by $\sim 80 \%$ while syt9 knockdown was $\sim 85 \%$. Rescue using a syt1 plasmid resulted in a recovery that was only $20 \%$ reduced from the wild-type. (D) CAPS1/2 knockdown depleted CAPS1 by $\sim 95 \%$ and CAPS2 by $\sim 90 \%$. A CAPS1 plasmid resulted in a rescue that was about $20 \%$ reduced from wild-type CAPS1 levels. This figure was adapted from (Kreutzberger, et al., Submitted). 
Synaptotagmin Effect

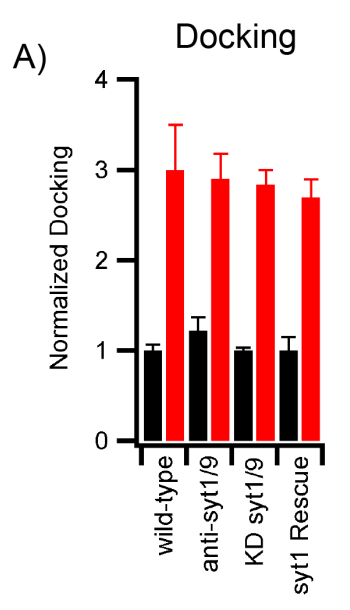

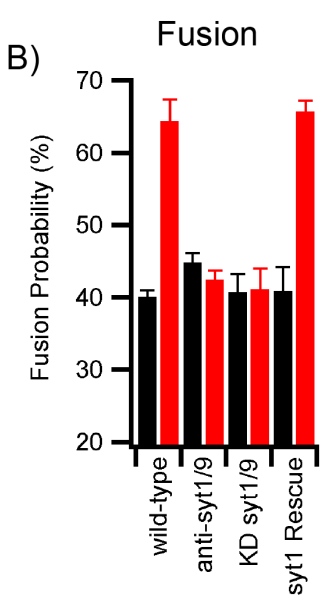

CAPS Effect

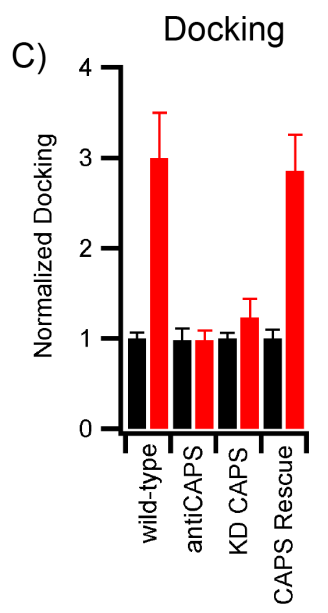

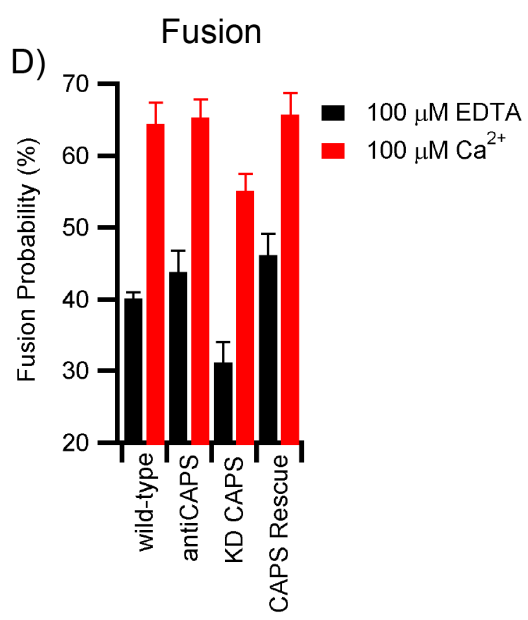

Figure 8.6: The role of synaptotagmin (A, B) and CAPS (C, D) on DCV docking and fusion in the presence of $100 \mu \mathrm{M}$ EDTA (black) or $100 \mu \mathrm{M} \mathrm{Ca}^{2+}$ (red). Wild-type preparations of DCVs were compared to those that were either treated with function blocking antibodies or to those that were purified from cells subjected to shRNA-mediated knockdown. In the latter case, expression of RNAi-resistant syt1 or CAPS-1 was used as a control. Black and red bar data were obtained in the absence and presence of $\mathrm{Ca}^{2+}$, respectively. Table 8.7 contains a summary of events and Table 8.8 contains fitting results. As indicated under Methods, docking values for preparations of DCVs from wild-type, knockdown, and RNAi rescue cell lines were individually normalized to the value obtained in the presence of $100 \mu \mathrm{M}$ EDTA enabling comparison of the relative effects elicited by calcium among the different preparations. This strategy does not enable us to rule out possible effects of synaptotagmin or CAPS knockdown on docking in the absence of calcium. For antibodytreated samples, we observed no significant effect on docking in the absence of calcium. This figure was adapted from (Kreutzberger, et al., Submitted). 
Strikingly, blocking CAPS function using an antibody that binds near the $\mathrm{PH}$ and $\mathrm{C} 2$ domains or shRNA-mediated CAPS depletion ( $>90 \%$ for both isoforms, Figure 8.5) gave results that significantly contrasted with those for synaptotagmin. Here the calcium-dependent increase of DCV docking was inhibited (Figure 8.6C and Table 8.7). For fusion, however, the CAPS antibody had no effect, whereas knockdown decreased the amount of fusion both in the absence and presence of calcium as compared to CAPS-containing DCVs (Figure 8.6D, Table 8.7, and Table 8.8). CAPS deficiency also substantially increased the delay time between docking and fusion (Figure 8.7B) likely indicating a role for CAPS in SNARE assembly. Co-expression of RNAi-resistant CAPS-1 during knockdown rescued the effects on both docking and fusion (Figure 8.6C, D, Table 8.7, and Table 8.8). Interestingly, as shown by the cumulative distribution for elapsed time between docking and fusion, synaptotagmin knockdown had no other kinetic effect through it blocked calcium-accelerated fusion (Figure 8.8A).
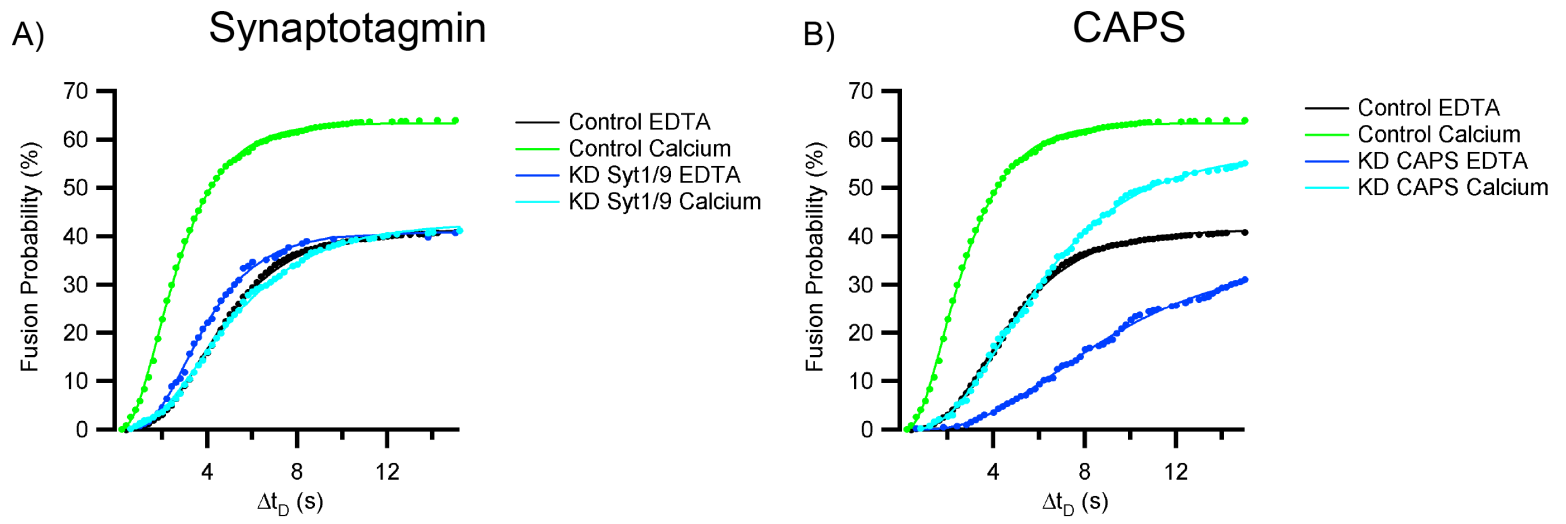

Figure 8.7: Cumulative distribution functions of the delay time between docking and fusion of DCVs from knockdowns that deplete synaptotagmin (A) and CAPS (B). Summary of fitting results are shown in Table 8.8. This figure was adapted from (Kreutzberger, et al., Submitted). 


\begin{tabular}{cccccc}
\hline Condition & $\begin{array}{c}\text { Number of } \\
\text { Experiments }\end{array}$ & $\begin{array}{c}\text { Normalized } \\
\text { Docking }\end{array}$ & $\begin{array}{c}\text { Percent } \\
\text { Fusion }\end{array}$ & $\begin{array}{c}\text { Total Number of } \\
\text { Docking Events }\end{array}$ & $\begin{array}{c}\text { Total Number of } \\
\text { Fusion Events }\end{array}$ \\
\hline Wild-type EDTA & 14 & $1.0 \pm 0.1$ & $41.0 \pm 0.9$ & 823 & 340 \\
Wild-type Ca ${ }^{2+}$ & 7 & $3.0 \pm 0.5$ & $64 \pm 3$ & 1753 & 1184 \\
Anti-syt1/9 EDTA & 5 & $1.2 \pm 0.2$ & $45 \pm 1$ & 244 & 108 \\
Anti-syt1/9 Ca ${ }^{2+}$ & 5 & $2.9 \pm 0.3$ & $42 \pm 1$ & 769 & 335 \\
KD syt1/9 EDTA & 5 & $1.00 \pm 0.03$ & $41 \pm 3$ & 234 & 98 \\
KD syt1/9 Ca ${ }^{2+}$ & 5 & $2.8 \pm 0.2$ & $41 \pm 3$ & 574 & 236 \\
syt1 rescue EDTA & 5 & $1.0 \pm 0.2$ & $41 \pm 3$ & 225 & 93 \\
syt1 rescue Ca ${ }^{2+}$ & 5 & $2.6 \pm 0.2$ & $66 \pm 2$ & 585 & 384 \\
Anti-CAPS1/2 EDTA & 5 & $1.0 \pm 0.1$ & $44 \pm 3$ & 214 & 95 \\
Anti-CAPS1/2 Ca ${ }^{2+}$ & 5 & $1.0 \pm 0.1$ & $65 \pm 3$ & 311 & 202 \\
CAPSKD EDTA & 5 & $1.0 \pm 0.2$ & $31 \pm 3$ & 410 & 127 \\
CAPS KD Ca ${ }^{2+}$ & 5 & $1.2 \pm 0.2$ & $55 \pm 2$ & 504 & 278 \\
CAPS rescue EDTA & 5 & $1.0 \pm 0.1$ & $46 \pm 3$ & 259 & 118 \\
CAPS rescue Ca ${ }^{2+}$ & 5 & $2.9 \pm 0.4$ & $66 \pm 3$ & 741 & 495 \\
\hline
\end{tabular}

Table 8.7: Event statistics for DCV docking and fusion to planar supported bilayers with syntaxin1a (183-288):SNAP-25 (lipid:protein 3000) with wild-type DCVs, DCVs inhibited with antibodies for either synaptotagmin or CAPS, or DCVs that have had synaptotagmin or CAPS knocked down using shRNA prior to purification. Graphs of data are shown in Fig. 2. Docking for all conditions was normalized to the docking observed in the wild-type EDTA sample. This figure was adapted from (Kreutzberger, et al., Submitted).

\begin{tabular}{cccc}
\hline Condition & $\begin{array}{c}\text { Number of } \\
\text { Experiments }\end{array}$ & $\mathbf{k}\left(\mathbf{s}^{-1}\right)$ & $\mathbf{m}$ \\
\hline Control EDTA & 14 & $0.40 \pm 0.10$ & $4.8 \pm 0.2$ \\
Control Ca ${ }^{2+}$ & 7 & $0.63 \pm 0.02$ & $3.1 \pm 0.1$ \\
Anti-syt1/9 EDTA & 5 & $0.45 \pm 0.04$ & $6.0 \pm 1.0$ \\
Anti-syt1/9 Ca ${ }^{2+}$ & 5 & $0.47 \pm 0.02$ & $6.0 \pm 0.5$ \\
KD syt1/9 EDTA & 5 & $0.54 \pm 0.06$ & $5.1 \pm 0.8$ \\
KD syt1/9 Ca ${ }^{2+}$ & 5 & $0.35 \pm 0.02$ & $3.5 \pm 0.3$ \\
syt1 rescue EDTA & 5 & $0.31 \pm 0.04$ & $2.6 \pm 0.3$ \\
syt1 rescue Ca ${ }^{2+}$ & 5 & $0.45 \pm 0.02$ & $2.1 \pm 0.1$ \\
Anti-CAPS1/2 EDTA & 5 & $0.44 \pm 0.05$ & $3.9 \pm 0.6$ \\
Anti-CAPS1/2 Ca ${ }^{2+}$ & 5 & $0.54 \pm 0.04$ & $2.9 \pm 0.3$ \\
CAPS KD EDTA & 5 & $0.23 \pm 0.02$ & $4.2 \pm 0.5$ \\
CAPSKD Ca ${ }^{2+}$ & 5 & $0.31 \pm 0.02$ & $3.7 \pm 0.3$ \\
CAPS rescue EDTA & 5 & $0.40 \pm 0.04$ & $3.8 \pm 0.5$ \\
CAPS rescue Ca ${ }^{2+}$ & 5 & $0.53 \pm 0.03$ & $2.2 \pm 0.2$ \\
\hline
\end{tabular}

Table 8.8: Fit values of a parallel reaction model $\left(N(t)=N\left(1-e^{-k t}\right)^{m}\right.$ where $N$ is the fusion probability, $k$ is the rate, and $m$ is the number of parallel reactions occurring) for the cumulative distribution function of delay times between docking and fusion for single particle DCV events for conditions described in Fig. 2. DCVs were inhibited using antibodies for synaptotagmin or CAPS or prepared from synaptotagmin- or CAPS-deficient cell lines generated by RNAi. This figure was adapted from (Kreutzberger, et al., Submitted). 
The protein machinery necessary for fusion to respond to calcium appears to be present on the secretory vesicle (Kiessling, et al., 2013; Park, et al., 2012; Scheuner, et al., 1992; Scheuner \& Holz, 1994), but a large amount of SNARE-dependent fusion occurs in the absence of calcium ( $40 \%$, Figure 8.3B), which is consistent with the SNAREs representing the minimal fusion machinery (Weber, et al., 1998). Other proteins are well known to regulate the interaction between fusion partners in situ and to affect SNARE complex assembly. Thus, we sought to modify the initial hybrid system by replacing the truncated version of syntaxin-1a (residues 183288, containing the transmembrane and SNARE domains) with the full-length protein containing in addition the $\mathrm{N}$-terminal and $\mathrm{H}_{\mathrm{abc}}$ domains that are thought to regulate SNARE complex function. Further, we included the SNARE regulatory proteins, Munc18 and complexin-1, to test the effects on docking and fusion both in the absence and presence of calcium. The overall goals of this strategy were to suppress calcium-independent fusion and to distinguish separate states of vesicle docking and calcium-triggered fusion analogous to those that occur in situ. The soluble proteins Munc18 and complexin-1 are known to be essential in vivo, but it is debated how they positively or negatively regulate fusion (Jahn \& Fasshauer, 2012; Südhof, 2013). Munc18 interacts with the regulatory $\mathrm{H}_{\mathrm{abc}}$ domain and N-peptide of syntaxin-1a (Dulubova, et al., 1999; Misura, et al., 2000; Toonen \& Verhage, 2007), and its deletion completely blocks exocytosis (Verhage, et al., 2000). Recently, it was proposed that a Munc18 homolog serves as a template for SNARE zippering (Baker, et al., 2015). Complexin-1 has been reported to block spontaneous synaptic vesicle exocytosis in the absence of calcium while increasing synchronized release (Yang, et al., 2010; Yang, et al., 2013), and earlier in vitro studies claimed that it binds the fully assembled synaptic SNARE complex and serves as a clamp to prevent fusion in advance of calcium signaling (Giraudo, et al., 2006; Giraudo, et al., 2009). However, very recent 
observations show that complexin-1 may act more proximally by binding a binary complex of syntaxin-1a and SNAP-25 thereby reducing assembly of synaptobrevin-2 into the fully zippered SNARE complex (Zdanowicz, et al., In Press). As a consequence of these observations, Munc18 and complexin-1 were our first priorities for addition during reconstitution.

In our hybrid assay, docking and fusion differed depending on whether the supported bilayers were reconstituted with a truncated syntaxin-1a (residues 183-288), or full-length syntaxin-1a (residues 1-288). As compared to truncated syntaxin-1a full-length syntaxin-1a reduced docking in the absence of calcium and reduced fusion in both the absence and presence of calcium (Figure 8.8 A-D, No Additions columns). When Munc18 was added to the assay, it had little effect on docking or fusion to bilayers containing truncated syntaxin-1a (Figure 8.8A, B). However, it increased docking in the absence of calcium and increased fusion in both the absence and presence of calcium when the bilayers contained full-length syntaxin-1a (Figure 8.8C, D), consistent with its ability to bind the full-length protein. When complexin-1 was added to our assay (without Munc18), docking was abolished in the absence of calcium regardless of which syntaxin-1a construct was present (Figure 8.8A, C). Inclusion of calcium with complexin1 enabled docking and fusion for both truncated and full-length syntaxin-1a, although fusion was decreased when the full-length syntaxin-1a was used (Figure 8.8B, D). When Munc18 and complexin-1 were added together in assays containing truncated syntaxin-1a, docking was blocked in the absence of calcium but both docking and fusion occurred in the presence of calcium (Figure 8.8A, B). Notably and most importantly, when full-length syntaxin-1a was used, addition of Munc18 and complexin-1 allows DCV docking but no fusion in the absence of 
calcium whereas the inclusion of calcium allows fusion to occur readily (Figure 8.8C, D). This last scenario reconstitutes docking and fusion as events that are separable by calcium dependence under full control of this key set of regulatory proteins and using conditions closely approximating those that occur physiologically. This accomplishment has not been achieved before.

Truncated syx (183-288)

A)

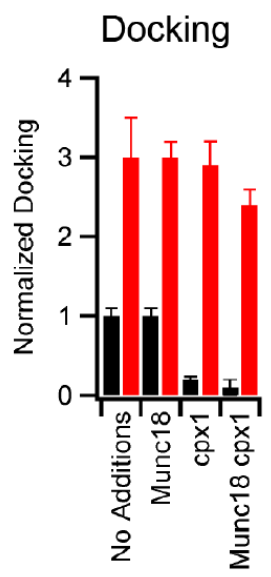

B)

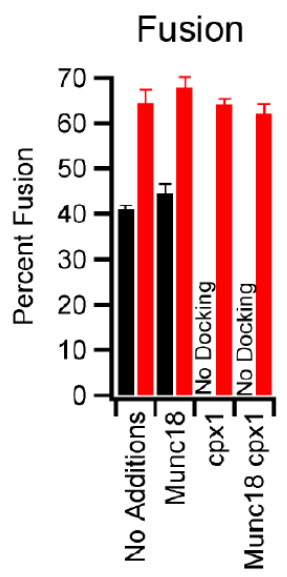

C)

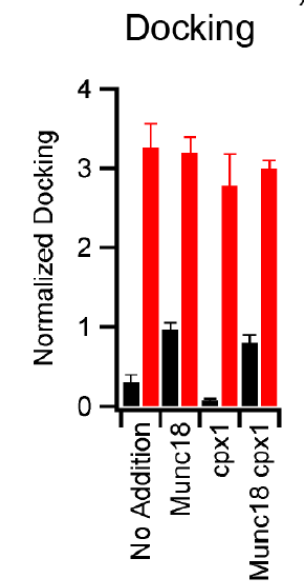

Full-length syx (1-288)

D)

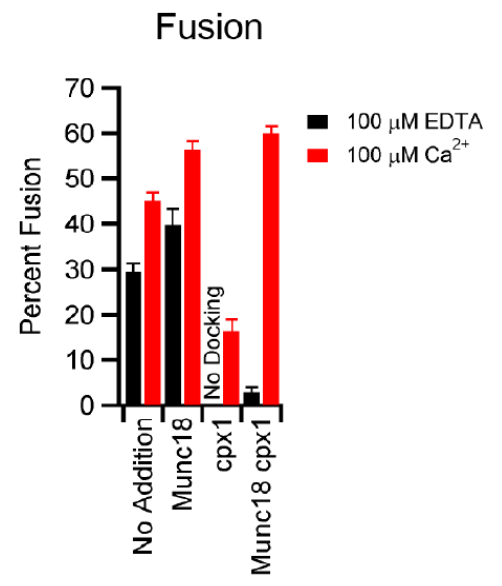

Figure 8.8: Docking and fusion probability for DCVs to planar supported bilayers containing truncated syntaxin-1a (183-288):SNAP-25 (A, B) or full-length syntaxin-1a (1-288):SNAP-25 (C, D) in the presence of $0.5 \mu \mathrm{M}$ Munc18, $2 \mu \mathrm{M}$ complexin-1, or both. Black and red bar data were obtained in absence and presence of $\mathrm{Ca}^{2+}$, respectively. Summary of events and fitting results are presented in Tables 8.9 and 8.10. Western blots show that Munc18 and complexin-1 were not detectable in DCV-enriched fractions prepared by centrifugation (Figure 8.9). This figure was adapted from (Kreutzberger, et al., Submitted). 


\begin{tabular}{|c|c|c|c|c|c|c|}
\hline & Condition & $\begin{array}{c}\text { Number of } \\
\text { Experiments }\end{array}$ & $\begin{array}{l}\text { Normalized } \\
\text { Docking }\end{array}$ & $\begin{array}{l}\text { Percent } \\
\text { Fusion }\end{array}$ & $\begin{array}{l}\text { Total Number of } \\
\text { Docking Events }\end{array}$ & $\begin{array}{l}\text { Total Number of } \\
\text { Fusion Events }\end{array}$ \\
\hline \multirow{8}{*}{ 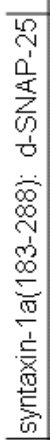 } & EDTA & 14 & $1.0 \pm 0.1$ & $40.1 \pm 0.9$ & 823 & 340 \\
\hline & $\mathrm{Ca}^{2+}$ & 7 & $3.0 \pm 0.5$ & $64 \pm 3$ & 1753 & 1184 \\
\hline & Munc18 EDTA & 5 & $1.0 \pm 0.1$ & $44 \pm 2$ & 376 & 167 \\
\hline & Munc18 Ca2+ & 5 & $3.0 \pm 0.2$ & $67 \pm 2$ & 957 & 651 \\
\hline & complexin-1 EDTA & 5 & $0.19 \pm 0.05$ & N/A & 38 & 2 \\
\hline & complexin-1 Ca2+ & 5 & $2.9 \pm 0.3$ & $64 \pm 1$ & 779 & 497 \\
\hline & $\begin{array}{c}\text { Munc18 complexin-1 } \\
\text { EDTA }\end{array}$ & 5 & $0.1 \pm 0.1$ & N/A & 39 & 8 \\
\hline & $\begin{array}{c}\text { Munc18 complexin-1 } \\
\text { Ca2+ }\end{array}$ & 5 & $2.4 \pm 0.2$ & $62 \pm 2$ & 680 & 422 \\
\hline \multirow{8}{*}{ 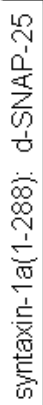 } & EDTA & 5 & $0.3 \pm 0.1$ & $30 \pm 2$ & 160 & 49 \\
\hline & $\mathrm{Ca}^{2+}$ & 5 & $3.3 \pm 0.3$ & $45 \pm 2$ & 766 & 342 \\
\hline & Munc18 EDTA & 5 & $1.0 \pm 0.1$ & $40 \pm 4$ & 333 & 142 \\
\hline & Munc18 Ca2+ & 5 & $3.2 \pm 0.2$ & $56 \pm 2$ & 473 & 268 \\
\hline & complexin-1 EDTA & 5 & $0.08 \pm 0.02$ & N/A & 22 & 1 \\
\hline & complexin-1 $\mathrm{Ca} 2+$ & 5 & $2.8 \pm 0.4$ & $16 \pm 3$ & 718 & 119 \\
\hline & $\begin{array}{c}\text { Munc18 complexin-1 } \\
\text { EDTA }\end{array}$ & 5 & $0.8 \pm 0.1$ & $2 \pm 1$ & 203 & 6 \\
\hline & $\begin{array}{c}\text { Munc18 complexin-1 } \\
\text { Ca2+ }\end{array}$ & 5 & $3.0 \pm 0.1$ & $60 \pm 2$ & 456 & 274 \\
\hline
\end{tabular}

Table 8.9: Event statistics for DCV docking and fusion to planar supported bilayers containing either syntaxin-1a (183-288) or syntaxin-1a (1-288) with SNAP-25 (at lipid:protein of 3000) with the addition of complexin-1 and/or Munc18 in the presence of $100 \mu \mathrm{M}$ EDTA or $100 \mu \mathrm{M} \mathrm{Ca}{ }^{2+}$. Graphs of data is shown in Figure 3. Docking for all conditions was normalized to the docking observed for the syntaxin-1a (183-288):SNAP25 in EDTA sample. This figure was adapted from (Kreutzberger, et al., Submitted). 


\begin{tabular}{|c|c|c|c|c|}
\hline & Condition & $\begin{array}{l}\text { Number of } \\
\text { Experiments }\end{array}$ & $k\left(s^{-1}\right)$ & m \\
\hline \multirow{8}{*}{ 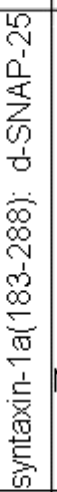 } & EDTA & 14 & $0.43 \pm 0.01$ & $4.4 \pm 0.2$ \\
\hline & $\mathrm{Ca}^{2+}$ & 7 & $0.63 \pm 0.02$ & $3.1 \pm 0.1$ \\
\hline & Munc18 EDTA & 5 & $0.38 \pm 0.03$ & $2.3 \pm 0.2$ \\
\hline & Munc18 $\mathrm{Ca}^{2+}$ & 5 & $0.56 \pm 0.02$ & $2.5 \pm 0.1$ \\
\hline & complexin-1 EDTA & 5 & -- & -- \\
\hline & complexin-1 $\mathrm{Ca}^{2+}$ & 5 & $0.57 \pm 0.02$ & $3.5 \pm 0.2$ \\
\hline & Munc18 complexin-1 EDTA & 5 & -- & -- \\
\hline & Munc18 complexin-1 $\mathrm{Ca}^{2+}$ & 5 & $0.58 \pm 0.02$ & $2.7 \pm 0.1$ \\
\hline \multirow{8}{*}{ 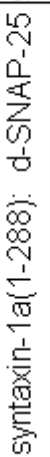 } & EDTA & 5 & $0.37 \pm 0.07$ & $4.0 \pm 0.9$ \\
\hline & $\mathrm{Ca}^{2+}$ & 5 & $0.43 \pm 0.02$ & $2.7 \pm 0.2$ \\
\hline & Munc18 EDTA & 5 & $0.51 \pm 0.04$ & $3.9 \pm 0.4$ \\
\hline & Munc18 $\mathrm{Ca}^{2+}$ & 5 & $0.56 \pm 0.03$ & $3.9 \pm 0.3$ \\
\hline & complexin-1 EDTA & 5 & -- & -- \\
\hline & complexin-1 $\mathrm{Ca}^{2+}$ & 5 & $0.36 \pm 0.04$ & $2.6 \pm 0.3$ \\
\hline & Munc18 complexin-1 EDTA & 5 & -- & -- \\
\hline & Munc18 complexin-1 $\mathrm{Ca}^{2+}$ & 5 & $0.59 \pm 0.03$ & $3.8 \pm 0.3$ \\
\hline
\end{tabular}

Table 8.10: Fit values of a parallel reaction model $\left(\left(N(t)=N\left(1-e^{-k t}\right)^{m}\right.\right.$ where $N$ is the fusion probability, $k$ is the rate, and $m$ is the number of parallel reactions occurring) for the cumulative distribution function of delay times between docking and fusion for single particle DCV events for conditions described in Figure 8.8. This figure was adapted from (Kreutzberger, et al., Submitted). 


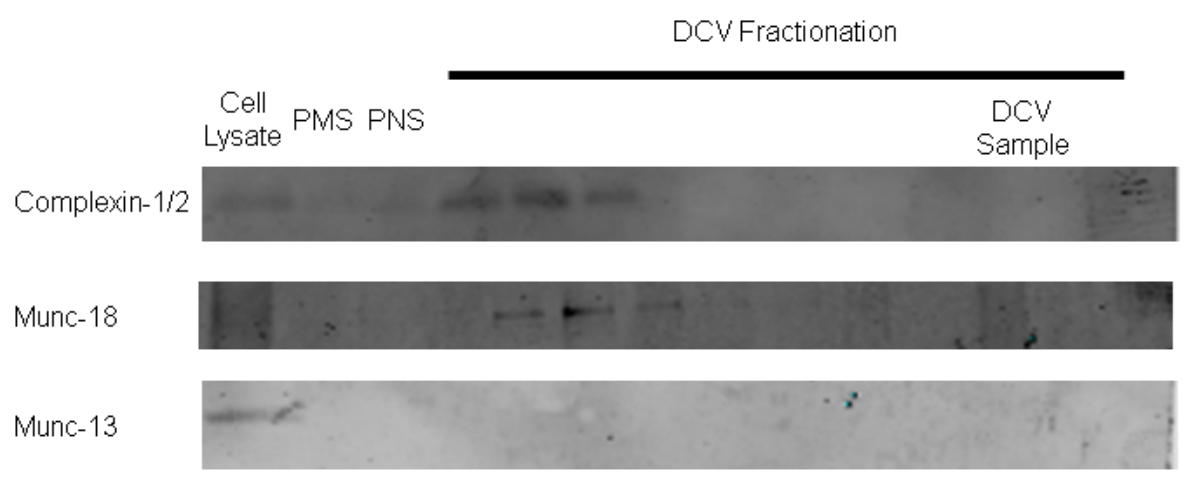

Figure 8.9: Western blots of complexin-1/2, Munc18, and Munc13 in fractions generated during DCV purification. DCV Sample identifies fraction 9 of the Optiprep gradient, which corresponds to fraction 9 in Figure 1A. Both complexin 1/2 and Munc18 are detected in lower density gradient fractions but not in the DCV Sample. Munc13 also is not present in the DCV Sample and is detected only in the original cell lysate (left-most lane). Most of Munc13 may have been pelleted with larger membranes during velocity sedimentation used to generate the PMS (post mitochondrial supernatant). PNS marks the post nuclear supernatant. This figure was adapted from (Kreutzberger, et al., Submitted).

In complementing this demonstration, we used various combinations of syntaxin-1a, SNAP-25, Munc18, complexin-1 to reveal that syntaxin-1a and Munc18 were sufficient to dock DCVs in the absence of SNAP-25, albeit at levels that were decreased compared to when both plasma membrane SNAREs were present. Further, we showed that the soluble synaptobrevin-2 (1-96) inhibitor peptide blocked this docking as well as that occurring with both Munc18 and complexin-1 present, thereby indicating that reconstituted docking is synaptobrevin-2 dependent (Figure 8.10). This outcome supports the prospective role of Munc18 in dual binding to syntaxin-1a and synaptobrevin-2, which may be analogous to the roles of Munc18's yeast homolog Vps33 (Baker, et al., 2015). 
Moreover, addition of complexin-1 in the absence of Munc18 and calcium inhibits docking under conditions with the truncated syntaxin-1a (183-288):SNAP-25 or the full-length syntaxin1a (1-288):SNAP-25 complexes (Figure 8.8), which is in agreement with recent results showing that complexin binds quite strongly to the plasma membrane SNAREs that are assembled in a strict 1:1 stoichiometry and thus blocks the interaction with synaptobrevin-2 (Zdanowicz, et al., In Press). Interestingly, in the presence of Munc-18 there is no effect of complexin-1 on docking to binary syntaxin-1a (1-288):SNAP-25 complexes, while fusion in the absence of calcium is dramatically inhibited by complexin-1 and this response depends strongly on increasing concentrations of complexin (Figure 8.8C and D, Figure 8.11). Therefore, Munc18 likely serves as a template for SNARE assembly allowing DCVs to be docked while complexin-1 acts within this complex to prevent SNARE zippering in the absence of calcium.

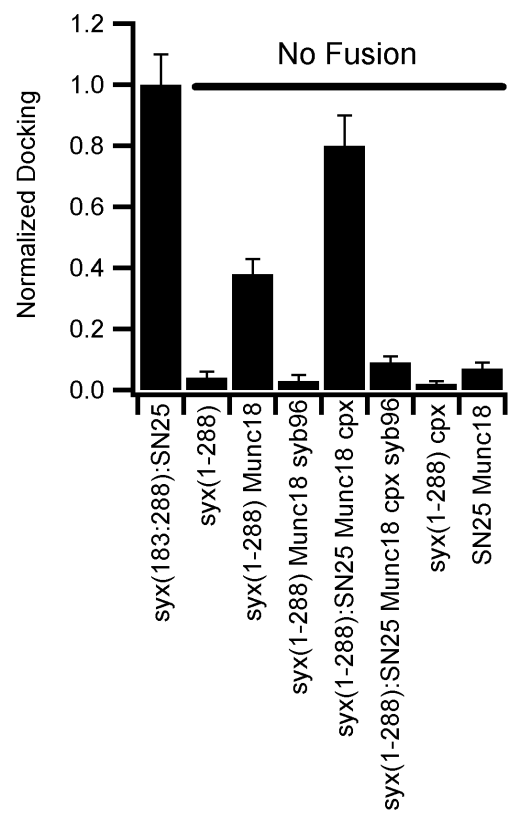

Figure 8.10: Controls to determine the effects of Munc18 and complexin-1 on docking to full-length syntaxin-1a (1-288) and SNAP-25 and to distinguish which of these conditions are inhibited by the soluble domain of synaptobrevin-2 (residues 1-96). Statistics are shown in Table 8.11. This figure was adapted from (Kreutzberger, et al., Submitted). 


\begin{tabular}{cccccc}
\hline Condition & $\begin{array}{c}\text { Number of } \\
\text { Experiments }\end{array}$ & $\begin{array}{c}\text { Normalized } \\
\text { Docking }\end{array}$ & $\begin{array}{c}\text { Percent } \\
\text { Fusion }\end{array}$ & $\begin{array}{c}\text { Total Number } \\
\text { of Docking } \\
\text { Events }\end{array}$ & $\begin{array}{c}\text { Total Number } \\
\text { of Fusion } \\
\text { Events }\end{array}$ \\
\hline $\begin{array}{c}\text { syx(183-288):dSN25 } \\
\text { syx(1-288) }\end{array}$ & 14 & $1.0 \pm 0.1$ & 41.0 & 823 & 340 \\
syx(1-288), Munc18 & 5 & $0.04 \pm 0.02$ & -- & 17 & 1 \\
$\begin{array}{c}\text { syx(1-288), Munc18, } \\
\text { Syb96 }\end{array}$ & 5 & $0.38 \pm 0.5$ & -- & 173 & 4 \\
$\begin{array}{c}\text { syx(1-288):dSN25, } \\
\text { Munc18, cpx }\end{array}$ & 5 & $0.03 \pm 0.02$ & -- & 15 & 1 \\
$\begin{array}{c}\text { Syx(1-288):dSN25, } \\
\text { Munc18, cpx, Syb96 }\end{array}$ & 5 & $0.09 \pm 0.02$ & -- & 203 & 6 \\
$\begin{array}{c}\text { syx(1-288), cpx } \\
\text { dSN25, Munc18 }\end{array}$ & 5 & $0.01 \pm 0.01$ & -- & 41 & 1 \\
\hline
\end{tabular}

Table 8.11: Event statistics for DCV docking and fusion to planar supported bilayers with the indicated combination of proteins syntaxin-1a (183-288), syntaxin-1a (1-288), and SNAP-25 (lipid:protein 3000) incubated, as indicated, with Munc18 (0.5 $\mu \mathrm{M})$, complexin-1 (2 $\mu \mathrm{M})$, and synaptobrevin-2 (1-96) inhibitor peptide. All conditions were measured in the presence of $100 \mu \mathrm{M}$ EDTA. Docking has been normalized to the docking observed in the syntaxin-1a (183-288):dSN25 sample. This figure was adapted from (Kreutzberger, et al., Submitted).

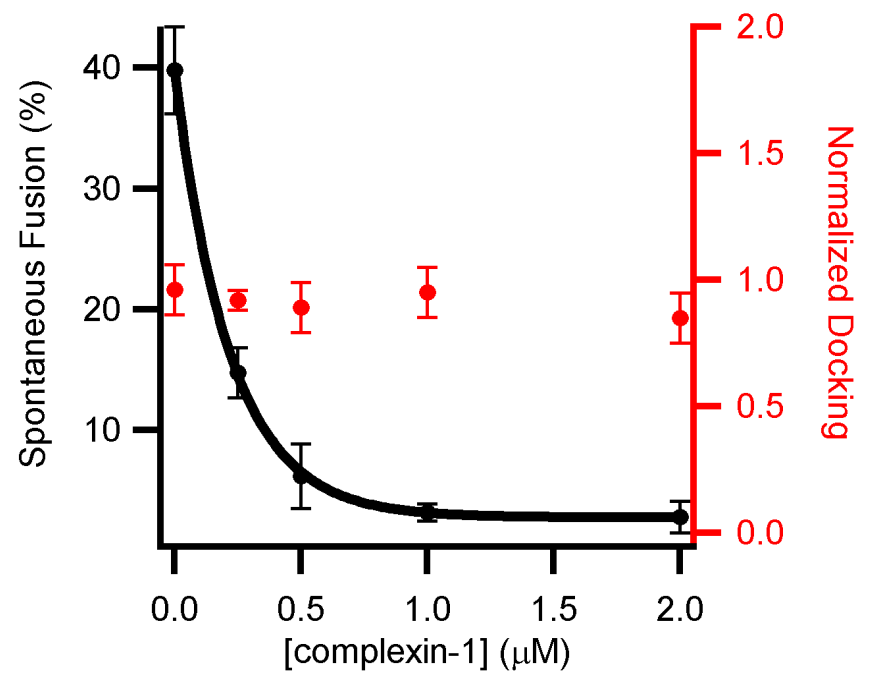

Figure 8.11: Complexin-1 inhibits fusion to planar supported bilayers containing syntaxin-1a (1288):SNAP-25 in the presence of $0.5 \mu M$ Munc18 in the absence of calcium while there is no effect on DCV docking. Table 8.12 contains a summary of events. This figure was adapted from (Kreutzberger, et al., Submitted). 


\begin{tabular}{cccccc}
\hline Condition & $\begin{array}{c}\text { Number of } \\
\text { Experiments }\end{array}$ & $\begin{array}{c}\text { Normalized } \\
\text { Docking }\end{array}$ & $\begin{array}{c}\text { Percent } \\
\text { Fusion }\end{array}$ & $\begin{array}{c}\text { Total Number } \\
\text { of Docking } \\
\text { Events }\end{array}$ & $\begin{array}{c}\text { Total Number } \\
\text { of Fusion } \\
\text { Events }\end{array}$ \\
\hline $0 \mu \mathrm{M} \mathrm{cpx}$ & 5 & $1.0 \pm 0.1$ & $40 \pm 5$ & 313 & 142 \\
$0.25 \mu \mathrm{M} \mathrm{cpx}$ & 4 & $0.92 \pm 0.04$ & $15 \pm 2$ & 147 & 22 \\
$0.5 \mu \mathrm{M} \mathrm{cpx}$ & 5 & $0.9 \pm 0.0 .1$ & $6 \pm 3$ & 235 & 15 \\
$1 \mu \mathrm{M} \mathrm{cpx}$ & 5 & $1.0 \pm 0.1$ & $3 \pm 1$ & 252 & 8 \\
$2 \mu \mathrm{M} \mathrm{cpx}$ & 5 & $0.8 \pm 0.1$ & $3 \pm 1$ & 203 & 6 \\
\hline
\end{tabular}

Table 8.12: Event statistics for DCV docking and fusion to planar supported bilayers containing syntaxin-1a (1-288):SNAP-25, $0.5 \mu M$ Munc18, and indicated amounts of complexin-1. Docking has been normalized to that observed in the $0 \mu \mathrm{M}$ complexin sample. This figure was adapted from (Kreutzberger, et al., Submitted).

Within cells, a subset of secretory vesicles exists in a readily releasable pool where vesicles are docked at the plasma membrane and primed to undergo rapid fusion in response to elevated levels of calcium (Martin, 2003; Sorensen, 2004). The condition where DCVs are docked on planar supported bilayers containing syntaxin-1a (1-288) and lipid-anchored SNAP-25 in the presence of Munc18 and complexin-1 but in the absence of calcium likely mimics this primed releasable state. To confirm that these DCVs are fusion competent, we first allowed them to dock under these conditions for $\sim 30$ minutes and then washed extensively with buffer containing the same amounts of Munc18 and complexin-1 to remove undocked DCVs. Then we added buffer containing calcium (concentration as indicated in figures) and a fluorescent dye (Cy5) to track calcium-containing buffer arrival, which allowed the ensuing calcium-dependent fusion kinetics to be monitored (Figure 8.12A). Calcium increased the rate and probability of docked DCV fusion thus serving as an authentic trigger (Figure 8.12B and Figure 8.13). The observed fusion kinetics from the arrested state (Figure 8.12B) was notably different from the kinetics 
observed between docking and fusion in the un-arrested pathway (fit of data in Figure 8.8D shown in Table 8.10). The kinetics of proceeding from docking to fusion showed a delay that is characteristic of the presence of intermediate(s) (Domanska, et al., 2009) while the calciumtriggered fusion followed first order kinetics indicating that the final intermediate had already been populated. 
A)

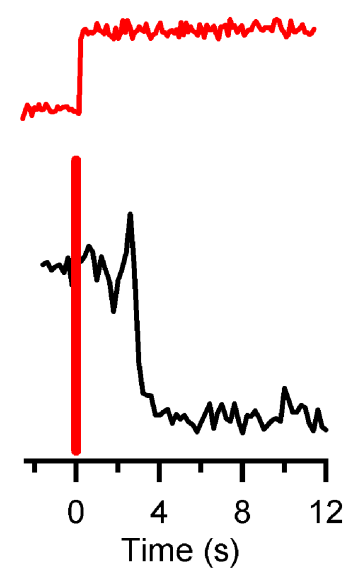

B)

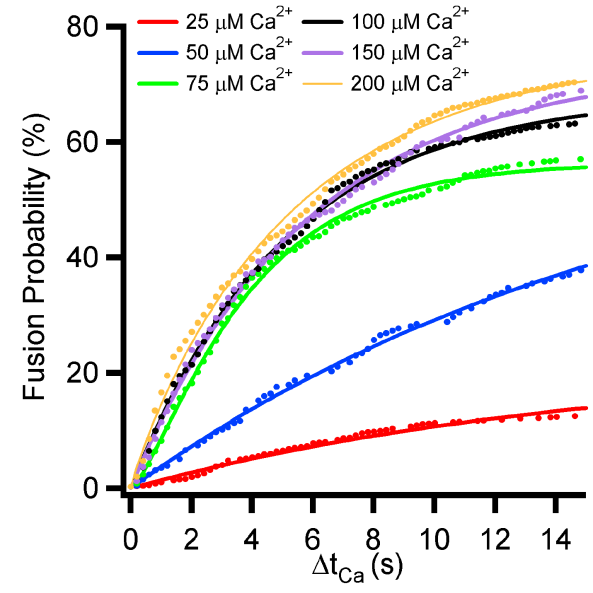

C)
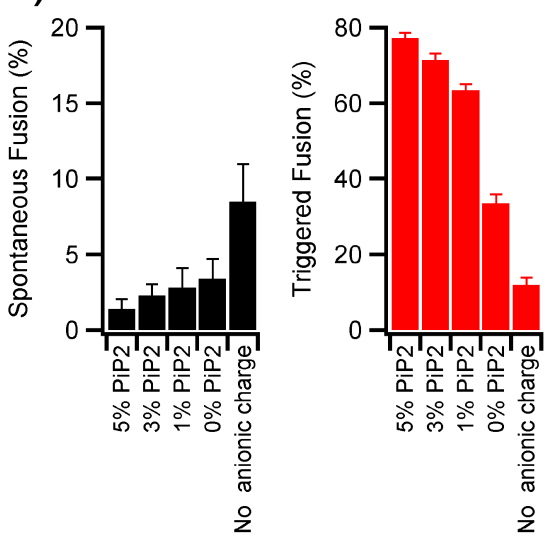

E)
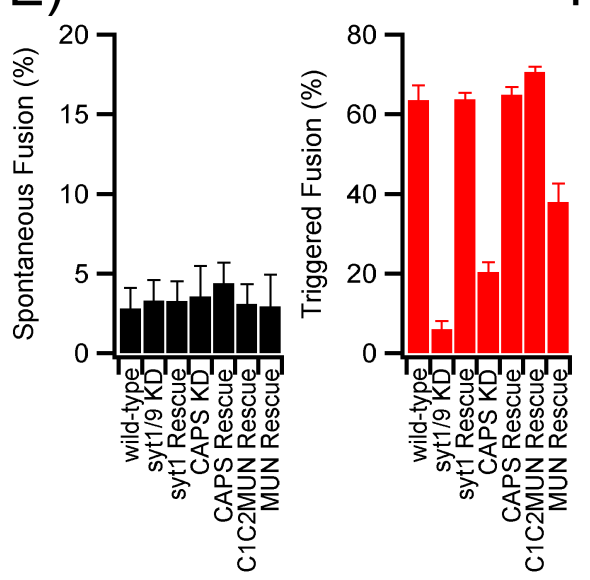

D)

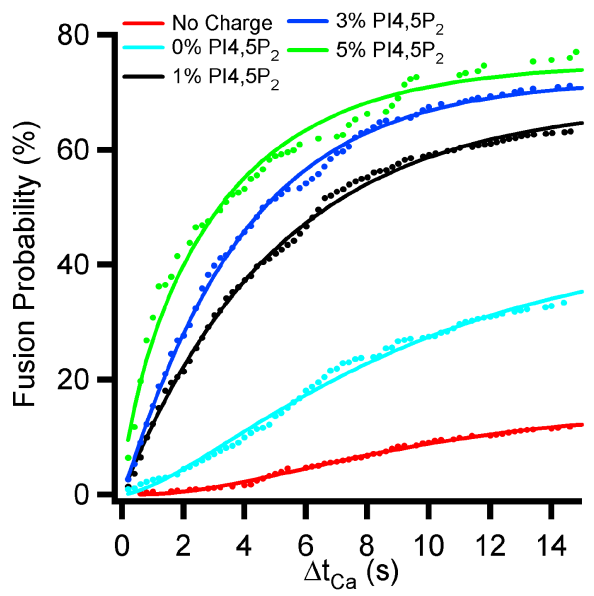

F)

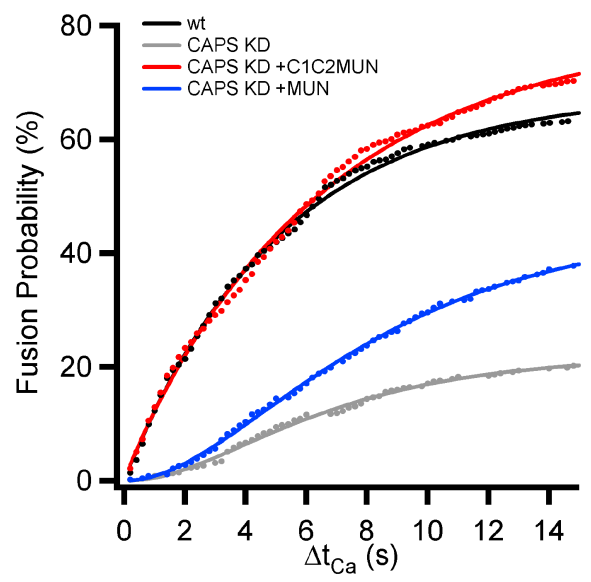


Figure 8.12: (A) Intensity trace of a single DCV calcium-triggered fusion event (black line) for a DCV docked to a planar supported bilayer in the presence of $0.5 \mu \mathrm{M}$ Munc18 and $2 \mu \mathrm{M}$ complexin1. Fusion was triggered with buffer $(120 \mathrm{mM}$ potassium glutamate, $20 \mathrm{mM}$ potassium acetate, 20 mM HEPES, pH 7.4) containing $\mathrm{Ca}^{2+}$. A soluble fluorescent dye (Cy5) was added to the buffer as an indicator for $\mathrm{Ca}^{2+}$ arrival (red line). (B) Cumulative distribution function for the time delay of fusion following the arrival of calcium $\left(\Delta \mathrm{t}_{\mathrm{Ca}}\right)$ at different $\left[\mathrm{Ca}^{2+}\right]$. Summary of Data is shown in Table 8.13. Probability of triggering DCV fusion as a function of $\left[\mathrm{Ca}^{2+}\right]$ with $\mathrm{K}_{1 / 2}\left[\mathrm{Ca}^{2+}\right]$ of $40 \mu \mathrm{M}$ is shown in Figure 8.13. (C) The effect of $\mathrm{PI} 4,5 \mathrm{P}_{2}$ and the absence of membrane anionic charge on spontaneous fusion of DCVs in the absence of $\mathrm{Ca}^{2+}$ (black bars) or upon triggering of fusion with $100 \mu \mathrm{M} \mathrm{Ca}^{2+}$ (red bars). (D) The cumulative distribution function for calcium-triggered fusion as a function of $\mathrm{PI}(4,5) \mathrm{P}_{2}$ is shown with the summary of data for spontaneous and triggered fusion in Tables 8.14 and 8.15, respectively. (E) The effects on spontaneous fusion (black bars) or fusion triggered by $100 \mu \mathrm{M} \mathrm{Ca}^{2+}$ (red bars) of RNAi-mediated knockdowns of synaptotagmin and CAPS, of corresponding knockdown/rescue using RNAi-resistant constructs and of addition of recombinant Munc13-derived C1C2-MUN or the MUN domain alone. Summary of data for spontaneous and triggered fusion of knockdowns is shown in Tables 8.16 and 8.17, respectively. (F) The cumulative distribution functions of fusion probability beginning at the time of $\mathrm{Ca}^{2+}$ arrival for DCVs from wild-type, CAPS knockdown, and CAPS knockdown rescued by the two Munc13 constructs. This figure was adapted from (Kreutzberger, et al., Submitted). 


\begin{tabular}{cccccc}
\hline Condition & $\begin{array}{c}\text { Number of } \\
\text { Experiments }\end{array}$ & Fusion Probability & $\begin{array}{c}\text { Docked } \\
\text { Events }\end{array}$ & $\begin{array}{c}\text { Triggered } \\
\text { Fusion }\end{array}$ & $\mathbf{k}\left(\mathbf{s}^{-1}\right)$ \\
\hline $25 \mathrm{M} \mathrm{Ca}^{2+}$ & 5 & $12 \pm 3$ & 541 & 61 & $0.06 \pm 0.01$ \\
$50 \mathrm{M} \mathrm{Ca}^{2+}$ & 5 & $38 \pm 4$ & 279 & 101 & $0.06 \pm 0.01$ \\
$75 \mathrm{M} \mathrm{Ca}^{2+}$ & 5 & $57 \pm 6$ & 913 & 534 & $0.110 \pm 0.006$ \\
$100 \mu \mathrm{M} \mathrm{Ca}^{2+}$ & 5 & $63 \pm 4$ & 723 & 462 & $0.190 \pm 0.007$ \\
$150 \mu \mathrm{M} \mathrm{Ca}^{2+}$ & 5 & $69 \pm 3$ & 425 & 291 & $0.194 \pm 0.008$ \\
$200 \mathrm{M} \mathrm{Ca}^{2+}$ & 5 & $70 \pm 2$ & 903 & 629 & $0.185 \pm 0.006$ \\
\hline
\end{tabular}

Table 8.13: Event statistics of triggered fusion of DCVs at different calcium concentrations from data shown in Figure 8.12B. The data are well described by a first order kinetic fit, $N(t)=N\left(1-e^{-k t}\right)$. This figure was adapted from (Kreutzberger, et al., Submitted). 


\begin{tabular}{lccccc}
\hline Condition & $\begin{array}{c}\text { Number of } \\
\text { Experiments }\end{array}$ & $\begin{array}{c}\text { Normalized } \\
\text { Docking }\end{array}$ & $\begin{array}{c}\text { Percent } \\
\text { Fusion }\end{array}$ & $\begin{array}{c}\text { Total Number of } \\
\text { Docking Events }\end{array}$ & $\begin{array}{c}\text { Total Number of } \\
\text { Fusion Events }\end{array}$ \\
\hline $5 \% \mathrm{Pl} 4,5 \mathrm{P}_{2}$ & 5 & $1.1 \pm 0.1$ & $1.4 \pm 0.6$ & 307 & 5 \\
$3 \% \mathrm{Pl} 4,5 \mathrm{P}_{2}$ & 5 & $1.0 \pm 0.1$ & $2.3 \pm 0.8$ & 264 & 6 \\
$1 \% \mathrm{Pl} 4,5 \mathrm{P}_{2}$ & 5 & $0.8 \pm 0.1$ & $3 \pm 1$ & 203 & 6 \\
$0 \% \mathrm{Pl} 4,5 \mathrm{P}_{2}$ & 5 & $0.8 \pm 0.1$ & $3 \pm 1$ & 208 & 7 \\
No Charge & 5 & $1.0 \pm 0.1$ & $8 \pm 3$ & 247 & 23 \\
\hline
\end{tabular}

Table 8.14: Event statistics of spontaneous fusion of DCVs docked in triggering conditions with planar supported bilayers of different lipid composition. Data are shown in Figure 8.12D. Docking has been normalized to the docking observed in the No Charge sample. This figure was adapted from (Kreutzberger, et al., Submitted).

\begin{tabular}{|c|c|c|c|c|c|c|}
\hline Condition & $\begin{array}{l}\text { Number of } \\
\text { Experiments }\end{array}$ & $\begin{array}{l}\text { Percent } \\
\text { Fusion }\end{array}$ & $\begin{array}{l}\text { Total Number of } \\
\text { Docking Events }\end{array}$ & $\begin{array}{l}\text { Total Number of } \\
\text { Fusion Events }\end{array}$ & $k\left(s^{-1}\right)$ & $m$ \\
\hline $5 \% \mathrm{Pl} 4,5 \mathrm{P}_{2}$ & 4 & $77 \pm 1$ & 196 & 151 & $0.26 \pm 0.04$ & $0.8 \pm 0.05$ \\
\hline $3 \% \mathrm{Pl} 4,5 \mathrm{P}_{2}$ & 5 & $71 \pm 2$ & 381 & 270 & $0.26 \pm 0.02$ & $1.0 \pm 0.1$ \\
\hline $1 \% \mathrm{Pl} 4,5 \mathrm{P}_{2}$ & 5 & $63 \pm 2$ & 723 & 462 & $0.23 \pm 0.02$ & $1.1 \pm 0.1$ \\
\hline $0 \% \mathrm{Pl} 4,5 \mathrm{P}_{2}$ & 4 & $33 \pm 2$ & 568 & 185 & $0.16 \pm 0.01$ & $1.9 \pm 0.1$ \\
\hline No Charge & 5 & $12 \pm 2$ & 804 & 103 & $0.17 \pm 0.03$ & $2.8 \pm 0.4$ \\
\hline
\end{tabular}

Table 8.15: Event statistics of triggered fusion of DCVs with planar supported bilayers of different lipid composition. All events were triggered with $100 \mu \mathrm{M} \mathrm{Ca}^{2+}$. Data are shown in Figure 8.12D. The data are well described by a first order kinetic fit, $N(t)=N\left(1-e^{-k t}\right)$. This figure was adapted from (Kreutzberger, et al., Submitted). 


\begin{tabular}{cccccc}
\hline Condition & $\begin{array}{c}\text { Number of } \\
\text { Experiments }\end{array}$ & $\begin{array}{c}\text { Normalized } \\
\text { Docking }\end{array}$ & $\begin{array}{c}\text { Percent } \\
\text { Fusion }\end{array}$ & $\begin{array}{c}\text { Total Number of } \\
\text { Docking Events }\end{array}$ & $\begin{array}{c}\text { Total Number of } \\
\text { Fusion Events }\end{array}$ \\
\hline Wild-type & 5 & $0.8 \pm 0.1$ & $3 \pm 1$ & 206 & 6 \\
syt1/9 KD & 5 & $0.9 \pm 0.1$ & $3 \pm 1$ & 227 & 8 \\
syt1 rescue & 5 & $0.8 \pm 0.1$ & $3 \pm 1$ & 335 & 12 \\
CAPSKD & 5 & $0.8 \pm 0.1$ & $4 \pm 2$ & 345 & 12 \\
CAPS1 rescue & 5 & $0.9 \pm 0.1$ & $4 \pm 1$ & 226 & 10
\end{tabular}

Table 8.16: Event statistics of spontaneous fusion of DCVs docked in triggering conditions with knockdowns of Syt1/9 or CAPS with subsequent recoveries. Data are shown in Figure 8.12E. This figure was adapted from (Kreutzberger, et al., Submitted).

\begin{tabular}{cccccc}
\hline Condition & $\begin{array}{c}\text { Number of } \\
\text { Experiments }\end{array}$ & $\begin{array}{c}\text { Fusion } \\
\text { Probability }\end{array}$ & $\begin{array}{c}\text { Docked } \\
\text { Events }\end{array}$ & $\begin{array}{c}\text { Triggered } \\
\text { Fusion }\end{array}$ & $\mathbf{k}\left(\mathbf{s}^{-\mathbf{1}}\right)$ \\
\hline Wild-type & 5 & $64 \pm 4$ & 723 & 462 & $0.190 \pm 0.007$ \\
syt1/9 KD & 5 & $6 \pm 2$ & 314 & 21 & -- \\
Syt1 rescue & 5 & $63 \pm 2$ & 227 & 145 & $0.189 \pm 0.005$ \\
CAPSKD & 5 & $20 \pm 3$ & 701 & 147 & $0.09 \pm 0.03$ \\
CAPS1 rescue & 5 & $65 \pm 2$ & 281 & 183 & $0.19 \pm 0.01$ \\
CAPSKD - C1C2Mun rescue & 5 & $70 \pm 2$ & 524 & 375 & $0.195 \pm 0.008$ \\
\hline
\end{tabular}

Table 8.17: Event statistics of triggered fusion of DCVs with knockdowns of syt1/9 or CAPS and subsequent recoveries. All events were triggered with $100 \mu \mathrm{M} \mathrm{Ca}^{2+}$. Data are shown in Figure 8.12E. The data are well described by a first order kinetic fit, $N(t)=N\left(1-e^{-k t}\right)$. This figure was adapted from (Kreutzberger, et al., Submitted). 


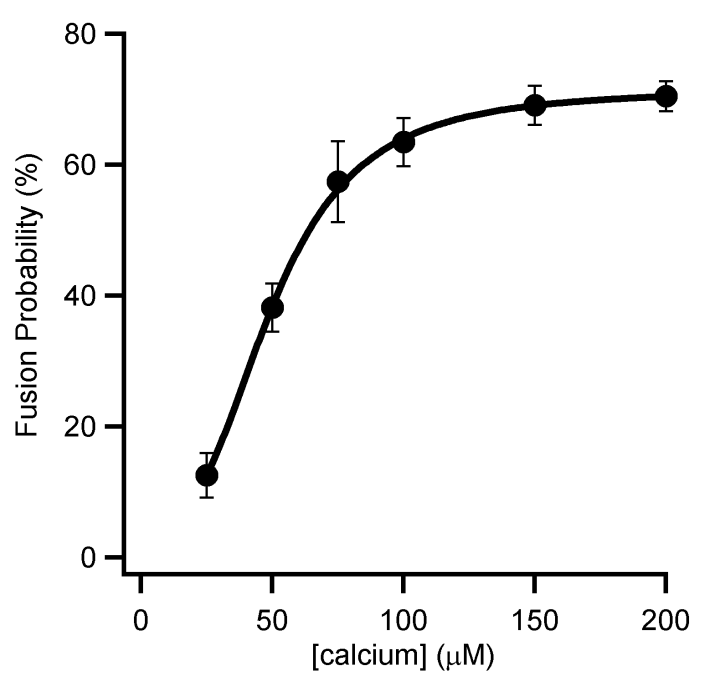

Figure 8.13: Fusion probability as a function of calcium for fusion of DCVs that were docked to planar supported bilayers with syntaxin-1a (1-288):SNAP-25 in the presence of Munc18 and complexin-1 upon triggering with calcium. The cumulative distribution functions, from which these data were derived, are shown in Figure 8.12B. This figure was adapted from (Kreutzberger, et al., Submitted).

Because PI4,5 $\mathrm{P}_{2}$ is a key constituent in priming of exocytosis (Holz \& Axelrod, 2002; Martin, 2012; Milosevic, et al., 2005), we investigated its effect on our reconstituted docking and triggered fusion as well as its influence on the function of the PI4,5 $\mathrm{P}_{2}$-binding proteins synaptotagmin and CAPS. The concentration of PI4,5 $\mathrm{P}_{2}$ included in the supported bilayer had no effect on spontaneous fusion in the absence of calcium but greatly enhanced triggered release

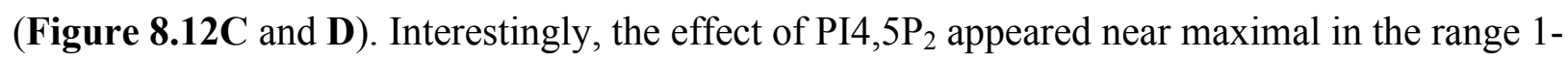
$5 \mathrm{~mol} \%$ of total lipid in the planar bilayer, which approximates the estimated plasmalemmal levels supporting DCV exocytosis in PC12 cells (James, et al., 2008). The absence of anionic charge (both PI4,5P $\mathrm{P}_{2}$ and PS) in the target membrane increased spontaneous fusion in the absence of calcium and abolished calcium-triggered fusion (Figure 8.12C). 
As a final issue in evaluating reconstituted two-step docking and fusion in the presence of regulatory proteins, we examined the effects of depleting synaptotagmin or CAPS from the DCVs. Loss of neither synaptotagmin nor CAPS appeared to affect spontaneous fusion (Figure 4E). However, without synaptotagmin, calcium-triggered fusion was completely inhibited while without CAPS, triggered fusion was greatly reduced (Figure 8.12E and $\mathbf{F}$ ). In the latter case, reduced fusion appears to be caused by a kinetic delay (evident in the cumulative distribution curve in Figure 8.12F) likely indicating a role of CAPS in priming of the fusion reaction. In situ, the function of CAPS may be closely coupled to or even partially overlap that of Munc13 (Kabachinski, et al., 2016), a protein that is essential for priming of synaptic fusion (Varoqueaux, et al., 2002). Thus, we were interested whether the reduced fusion incurred upon depletion of CAPS might be rescued by adding to our reconstitution a polypeptide containing the $\mathrm{C} 2$ and MUN domains that are common to CAPS and Munc13. For this purpose, we used purified recombinant polypeptides containing the Munc13 MUN domain alone or the Munc13 MUN domain preceded by the $\mathrm{C} 1$ and $\mathrm{C} 2$ domains (C1C2-MUN) of Munc13. As shown in Figure 4E and F, inclusion of MUN partially rescues, whereas C1C2-MUN fully rescues, the kinetic delay in fusion caused by the CAPS knockdown. This result indicates that we have repopulated the final (primed) step leading to fusion. While, under these precise conditions the Munc13 C1C2MUN domain seems to be interchangeable with CAPS in the kinetic priming of the docked DCV state, they are not redundant players in vivo and more detailed studies of the respective interactions and effects on priming will need to be addressed in the future. 


\subsection{Discussion}

Our hybrid system using neuroendocrine DCVs and planar supported bilayers has enabled us to reconstruct in vitro a stepwise docking, priming, and fusion process that bears a remarkable resemblance to exocytosis in situ and to clarify the roles of key supporting proteins. Using this approach, we have made the following new findings (see also Table 8:18 for more detail): 1) Munc18 and complexin-1 in the presence of syntaxin-1a (1-288) and SNAP-25 are able to arrest DCV in a docked state in the absence of calcium; 2) CAPS appears to kinetically increase the rate of the calcium response from this docked state while also being able to dock DCVs to $\mathrm{PI}, 5 \mathrm{P}_{2}$-containing membranes in response to calcium; 3) the $\mathrm{C} 1 \mathrm{C} 2-\mathrm{MUN}$ domain of Munc13 can replace CAPS priming under the conditions investigated here; and 4) the depletion of PI4,5 $\mathrm{P}_{2}$ kinetically reduces the rate of calcium triggering from the docked state in a manner that closely resembles the effects of CAPS knockdown.

These findings have led us to propose a model (Figure 8.14) where DCVs can dock to membranes in a stable non-fusing state when syntaxin-1a (1-288), SNAP-25, Munc18, and complexin-1 are present in a process that is dependent on synaptobrevin-2. CAPS can prime these docked DCVs to fuse efficiently when triggered with calcium. The actual fusion triggering is caused by synaptotagmin in a process that requires anionic lipid target membranes with a strong preference for PI4,5 $\mathrm{P}_{2}$. The calcium-dependent docking of DCVs facilitated by DCVassociated CAPS (Kabachinski, et al., 2016) could then function to recruit more DCVs to PI4,5P 2 containing membranes allowing more fusion events to occur (Figure 8.14). With respect to this last point, we are encouraged by earlier cellular studies showing calcium-dependent recruitment 
of synaptic vesicles after depletion of docked vesicles (Zenisek, et al., 2000) and similar behavior noted for DCVs (Karatekin, et al., 2008) that may depend at least in part on DCVassociated CAPS (Kabachinski, et al., 2016). The direct binding of calcium by CAPS $\left(\mathrm{K}_{\mathrm{d}} \sim 270\right.$ $\mu \mathrm{M})$ (Ann, et al., 1997) was similar for what was observed for calcium's $\mathrm{K}_{d}$ for docking in our reconstituted system $\left(\sim 240 \mu \mathrm{M}\right.$ in the presence of $\left.1 \% \mathrm{PI}, 5 \mathrm{P}_{2}\right)$. These calcium concentrations are well above the physiological level of intracellular calcium present under stimulatory conditions, but the docking had a large dynamic range with increases in DCV docking to planar supported bilayers observed under $100 \mu \mathrm{M}$ calcium. Previously, PI4,5P 2 was shown to be enriched around syntaxin-1a (van den Bogaart, et al., 2011) and act as a recruitment site for secretory vesicle docking (Honigmann, et al., 2013). Our results indicate that calcium-dependent DCV docking increased in the presence of higher concentrations of $\mathrm{PI} 4,5 \mathrm{P}_{2}$ suggesting that local enrichments of $\mathrm{PI} 4,5 \mathrm{P}_{2}$ around syntaxin-1 a could drive calcium-dependent CAPS-mediated docking to physiologically relevant calcium concentrations. Whether this is a meaningful correlation or the relatively high $\mathrm{K}_{\mathrm{d}}$ reflects a current limitation of our in vitro system will require further study. In the future, it will be important to determine if additional proteins that have been implicated in docking, e.g., granulphilin (Gomi, et al., 2005), rabphilin-3a, Munc13-4 (Chung, et al., 1998; Boswell, et al., 2012) as well as lipid micro domains will, when included in our analyses, reduce the calcium $\mathrm{K}_{\mathrm{d}}$ for vesicle docking to micromolar or even submicromolar levels.

The hybrid approach combining biological DCVs and reconstituted target membranes in a robust and efficient single vesicle fusion assay enabled us to dissect and assign precise mechanistic 
roles to a large number of molecules (and some of their sub-domains) that regulate calcium triggered exocytosis, which has previously eluded experimental confirmation. Our results have demonstrated that complexin, Munc18, and the regulatory Habc domain of syntaxin-1a are all necessary and sufficient to create a fusion-arrested acceptor SNARE complex capable of specifically docking secretory vesicles in the absence of calcium. CAPS or Munc13 coordinate the system to be primed for subsequent efficient calcium-triggered synaptotagmin-dependent fusion. The work also demonstrated that $\mathrm{PI} 4,5 \mathrm{P}_{2}$ in the target membrane plays critical roles in both enhancing CAPS/Munc13-dependent priming and synaptotagmin-dependent fusion and that the lipid context is of critical importance in defining the protein and lipid interactions that are physiologically relevant in exocytosis. Future work using this powerful system will certainly further refine the proposed model and permit further dissection of the specific interactions and interplays between the different protein modules and lipids that lead to calcium-triggered exocytosis. 


\begin{tabular}{|c|c|c|c|c|c|c|c|}
\hline \multirow{2}{*}{$\begin{array}{l}\text { Starting } \\
\text { Membrane } \\
\text { Condition }\end{array}$} & \multirow{2}{*}{$\begin{array}{l}\text { Vesicle } \\
\text { Condition' } \\
\text { Additions }\end{array}$} & \multicolumn{2}{|c|}{ Docking Probability } & \multicolumn{2}{|c|}{ Fusion Probability } & \multicolumn{2}{|c|}{$k_{\text {fusion }}$} \\
\hline & & EDTA & $\mathrm{Ca}^{2+}$ & EDTA & $\mathrm{Ca}^{2+}$ & EDTA & $\mathrm{Ca}^{2+}$ \\
\hline \multirow{5}{*}{ 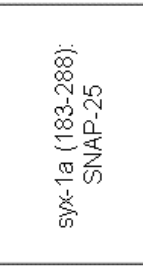 } & synaptotagmin & 0 & 0 & 0 & +++ & 0 & +++ \\
\hline & CAPS & 0 & +++ & + & + & + & + \\
\hline & complexin-1 & -- & 0 & no docking & 0 & no docking & 0 \\
\hline & Munc18 & 0 & 0 & 0 & 0 & + & + \\
\hline & $\mathrm{PI}(4,5) \mathrm{P} 2$ & 0 & +++ & 0 & +++ & 0 & +++ \\
\hline \multirow{2}{*}{ 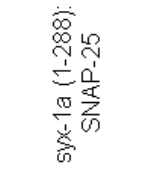 } & complexin-1 & -- & 0 & no docking & 0 & no docking & 0 \\
\hline & Munc18 & + & + & + & + & + & + \\
\hline \multirow{2}{*}{ 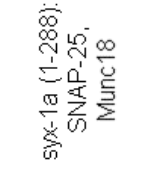 } & \multirow[b]{2}{*}{ complexin-1 } & \multirow[b]{2}{*}{0} & \multirow[b]{2}{*}{0} & \multirow[b]{2}{*}{---} & \multirow[b]{2}{*}{0} & \multirow[b]{2}{*}{--} & \multirow[b]{2}{*}{0} \\
\hline & & & & & & & \\
\hline \multirow{4}{*}{ 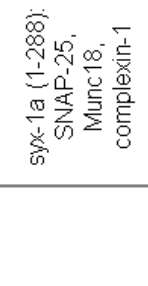 } & synaptotagmin & 0 & NiE & 0 & +++ & 0 & +++ \\
\hline & CAPS & 0 & NiE & 0 & + & 0 & +++ \\
\hline & $\mathrm{PI}(4,5) \mathrm{P} 2$ & 0 & NiE & 0 & + & 0 & +++ \\
\hline & $\begin{array}{ll}0: & \text { no } \\
+: & \text { pos } \\
+++: & \text { stro }\end{array}$ & $\begin{array}{l}\text { effect } \\
\text { itive effect } \\
\text { ng positive }\end{array}$ & & $\begin{array}{ll}-: & \text { ne } \\
---: & \text { str } \\
\text { NiE: : no } & \text { no }\end{array}$ & $\begin{array}{l}\text { ative effe } \\
\text { ng nega } \\
\text { examine }\end{array}$ & ve effect & \\
\hline
\end{tabular}

Table 8.18: Summary of results on the role of soluble and DCV-associated proteins and regulatory lipids obtained by the hybrid reconstitution approach of docking and fusion of single DCVs on planar supported bilayers with reconstituted SNAREs under different target membrane, vesicle membrane, and added protein conditions. This figure was adapted from (Kreutzberger, et al., Submitted). 


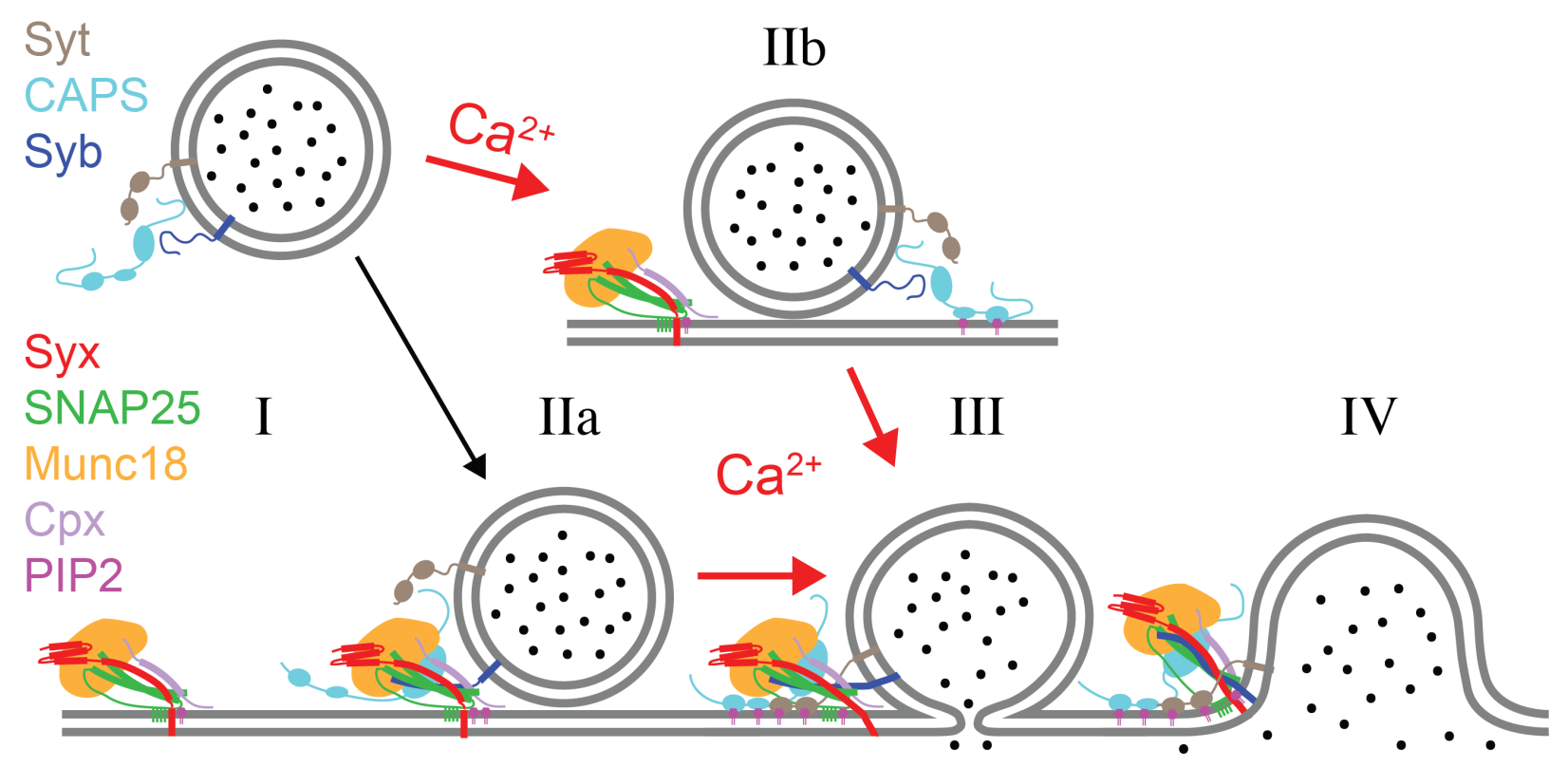

Figure 8.14: Model of DCV docking and fusion during exocytosis. Calcium-dependent release of DCV content requires an acceptor complex consisting of syntaxin-1a, SNAP25, Munc18, and complexin (complexin) as well as $\mathrm{PI} 4,5 \mathrm{P}_{2}$ in the plasma membrane. The complex interacts with synaptobrevin-2 and synaptotagmin in the vesicle membrane as well as CAPS that may or may not be vesicle-associated (I). DCVs are able to dock to the complex in the absence of calcium in a SNARE dependent fashion (IIa). The presence of complexin "clamps" the resulting pre-fusion (trans-SNARE) complex preventing progression to an open fusion pore and allows priming of the fusion machinery. Priming depends on CAPS and PI4,5 $\mathrm{P}_{2}$ and might involve a spatial organization of multiple copies of trans-SNARE complexes and accessory proteins as well as the organization of a specific local nanoscale lipid environment. The primed intermediate state resembles granules in the readily releasable pool in situ. Calcium influx triggers fusion pore opening catalyzed by synaptotagmin (III) and eventually the collapse of the vesicle membrane into the plasma membrane (IV). Calcium also facilitates CAPS-dependent docking of DCVs to the plasma membrane (IIb). These granules proceed through some intermediates that might resemble the priming steps in the absence of calcium to a fusion pore (III) and eventually to the complete merger of the two membranes (IV). Illustration was drawn by Volker Kiessling. This figure was adapted from (Kreutzberger, et al., Submitted). 


\section{Chapter 9: Conclusions and Outlook}

\subsection{Summary and Outlook}

The work presented in this thesis demonstrated the use of planar supported bilayers as a tool to study regulated exocytosis. Fusion of single proteoliposomes or physiological vesicles can be visualized using fluorescent membrane dyes or soluble content markers. These vesicles fuse with planar supported bilayers and release their content under the cleft of the planar bilayer. Using this assay, it was shown that preparing monomeric syntaxin-1a and getting it complexed with SNAP-25 in a 1:1 ratio is essential for efficient membrane fusion. This 1:1 plasma membrane SNARE complex can bind complexin-1 which inhibits the association of the vesicle SNARE synaptobrevin-2. When syntaxin-1a contains its regulatory Habc domain and N-peptide, Munc18 facilitates vesicle docking while complexin-1 inhibits fusion. Calcium stimulates these docked vesicles to fuse in a fashion that is strictly dependent on the protein synaptotagmin. Moreover, the MUN domain (from CAPS or Munc13) facilitates the kinetically fast response to calcium, which has been termed "priming" in vivo. The curvature of secretory vesicles likely plays a role in the speed and cooperativity of SNARE-dependent fusion, while cholesterol directly effects the probability of pore opening. Anionic lipids, especially $\mathrm{PI}(4,5) \mathrm{P}_{2}$ are required for coupling fusion to calcium through their interactions with synaptotagmin and possibly the priming proteins CAPS and/or Munc13.

Most studies of exocytosis focus on the central SNARE core molecular complex that together with the regulatory proteins facilitates membrane fusion in response to increases in calcium (Jahn \& Fasshauer, 2012). While the simplicity of this model is appealing, it likely cannot 
explain the diversity of secretory responses involved in cell-cell communication, which is necessary for multi-cellular organisms. Secretory vesicles are likely not a homogenous population of organelles but possess intrinsic molecular differences and differential interaction partners (Crawford \& Kavalali, 2015). In the studies presented in this thesis I focused on proteins such as synaptobrevin-2, syntaxin-1a, SNAP-25, synaptotagmin-1, and Mun13/CAPS. Several other SNARE proteins are known to function in regulated exocytosis in a functionally distinct manner. E.G. vesicle SNAREs such as synaptobrevin-1, VAMP4, and vtila function in synchronous release, asynchronous release, and spontaneous vesicle recycling, respectively (Schoch, et al., 2001; Raingo, et al., 2012; Walter, et al., 2014). The calcium sensor synaptotagmin is known to have 16 mammalian isoforms that bind calcium with different affinities which can lead to differential responses to calcium stimulation (Rao, et al., 2004), but a detailed understanding of the heterogeneity of calcium responses governed by synaptotagmin is still desired. The presence of many other proteins that can also bind calcium acting in similar or completely different roles complicates direct investigation of synaptotagmin's in cells which is a distinct advantage of the hybrid assay presented in this work. Chapter 8 showed the role of a kinetic priming that by CAPS or Munc13-1 (C1C2-MUN domain) to response rapidly to calcium but these proteins are not considered redundant in vivo (Martin, 2012). Complicating this matter is the fact that there are several more isoforms of Munc13 such as Munc13-2 and Munc13-4 that also play similar but not equivalent roles in vesicle priming (James \& Martin, 2013). A detailed comparison of these proteins in the reconstituted hybrid DCV assay would provide a great insight into the complexity of the exocytotic priming machinery. 


\section{A1: Membrane dye labeling of a physiological vesicle}

\section{Introduction}

The incorporation of physiological vesicles into ensemble and single particle fusion assays necessitates the need to labeled the vesicles membrane to allow observation/ read out of fusion. The incorporation of NPY-Ruby into the DCV assay removed the necessity of incorporating a membrane dye in these studies, but early attempts and failures at dye labeling occurred. Here, a discussion of methods attempted to labeled physiological vesicles will be discussed to assist future attempts of others in labeling physiological membranes.

\section{Observations}

Two classes of membrane dye were used in attempts to labeled physiological vesicles. The first was lipids with fluorescent head groups attached and the second was lipophilic tracers.

Fluorescently conjugated lipids (Rh-DOPE, R18, or DMPC-Alexa647) were placed glass tubes and dried down under a gentle stream of nitrogen gas then placed in a vacuum desiccator for $>1$ hour. After one hour vesicle samples were added to the tube and lightly vortexed. Steps of dialysis or centrifugation can be used to remove free dye, but if low concentration of dye is used these steps might not be necessary due to full incorporation of membrane dye into vesicle membrane. The lipophilic tracer dyes (DiI or DiD) only had marginal success with attempts to dry them down and solubilize the dye into the vesicle membrane. The Di-Dyes are not soluble in water and form aggregates/precipitate in solution when attempting to add them to physiological membranes. Some success was achieved by Sung-Tae Yang by adding a small volume of DiI or DiD in methanol directly to GPMV samples. Other possibilities include loading the Di-Dye into 
fatty acid depleted BSA which can bind the lipid like chains of the dye. Centrifugation at high speeds removed dye that was not successfully solubilized. Di-Dye's will spontaneously partition out of the BSA and into membranes.

\section{Attempts at membrane labeling DCVs}

All preliminary attempts to label DCVs by the BSA-DiD method and simultaneously monitory NPY-Ruby signal failed to observed fusion. The DiD did not appear to uniformly label vesicles and frequently it's signal would appear to dominate both the DiD channel and the Ruby channel of the optisplit, Figure A1.1. Decreasing the DiD added in the labeling process results in a few events where docking of DCVs could be observed with each fluorescent marker, Figure A1.2, but event at these concentrations no fusion events were observed. Personal communications from Bob Rawle (postdoctoral fellow of Peter Kasson and Steve Boxer) informed me that too much dye incorporation into influenza virus particles was inhibitory to fusion in his single particle fusion assay. Something similar could be occurring for DCVs and further systematic titration of DiD concentration might reveal how to solve labeling/fusion problem.

Attempts with lipids conjugated to fluorescent head groups yielded positive preliminary results. Drying down small amounts of Rh-DOPE and solubilizing it into DCVs resulted in observation of fusion events through the use of only the membrane dye, Figure A1.3. Using similar labeling conditions but switching the fluorescent lipid to DMPE-Alexa647 led to good labeling of the DCV sample, and simultaneous monitoring of both the membrane dye and NPY-Ruby (Figure 
A1.4) where docking and fusion could both be observed, Figure A1.5. Comparing the membrane dye label the DCV specific NPY-Ruby label revealed a large population of vesicles that were not labeled with NPY-Ruby. These are likely DCVs that were unsuccessfully labeled or contamination of other membrane compartments in the DCV preparation. The data used in the main body of this Thesis was only acquired with the specific content label making concerns of contamination irrelevant.

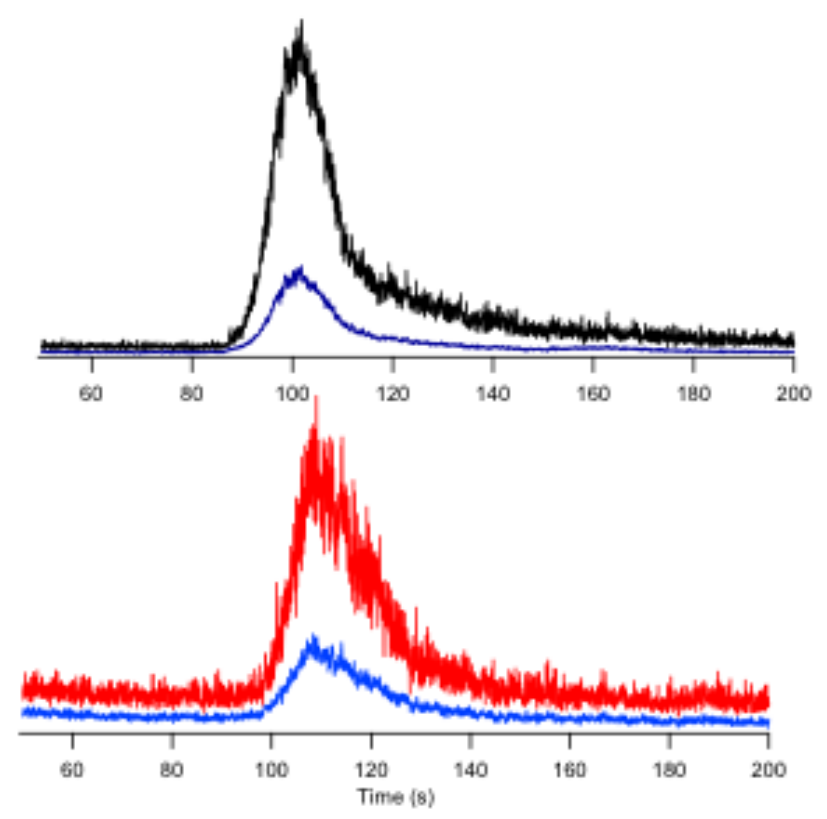

Figure A1.1: DCV transfected with NPY-Ruby (channel showed in lower panel) and labeled by the BSA-DiD method (DiD channel showed in the upper panel). The peak pixel (black traces for DiD or red traces for NPY-Ruby channel) and mean (lower traces) intensity traces are shown for both labeled channels. The DiD did not have uniform incorporation and some vesicles appear to have the DiD signal dominate both fluorescent channels as shown. $5 \mu \mathrm{L}$ of BSA-DiD was used for labeling. 

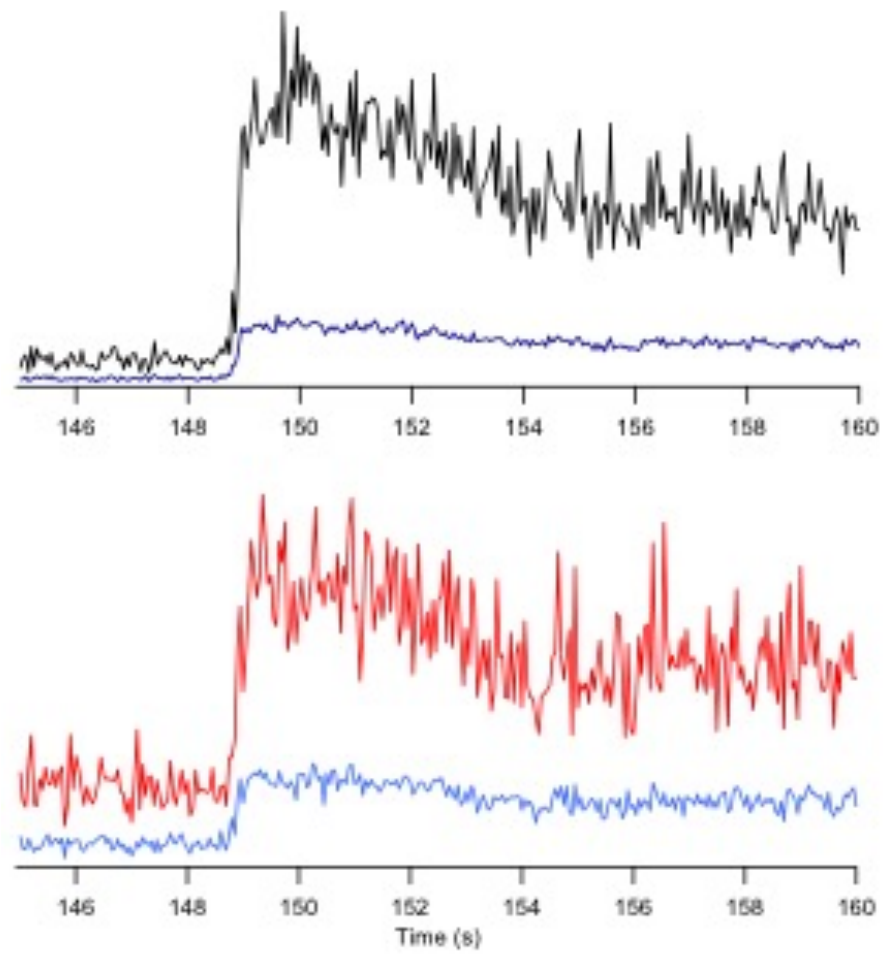

Figure A1.2: DCV transfected with NPY-Ruby (channel showed in lower panel) and labeled by the BSA-DiD method (DiD channel showed in the upper panel). The peak pixel (black traces for DiD or red traces for NPY-Ruby channel) and mean (lower traces) intensity traces are shown for both labeled channels. $0.5 \mu \mathrm{L}$ of BSA-DiD was used for labeling. 


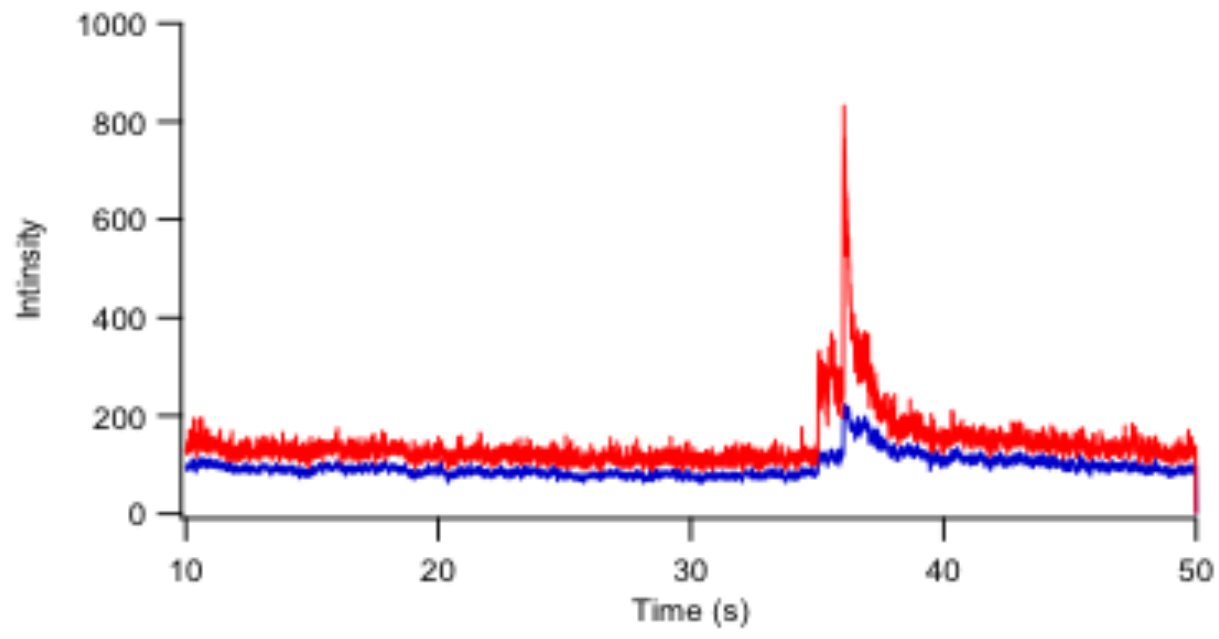

Figure A1.3: DCV labeled with Rh-DOPE. DCV were labeled by drying down $2 \mu \mathrm{L}$ of Rh-DOPE on glass and re-suspending in the presence of $100 \mu \mathrm{L}$ of DCV sample. 


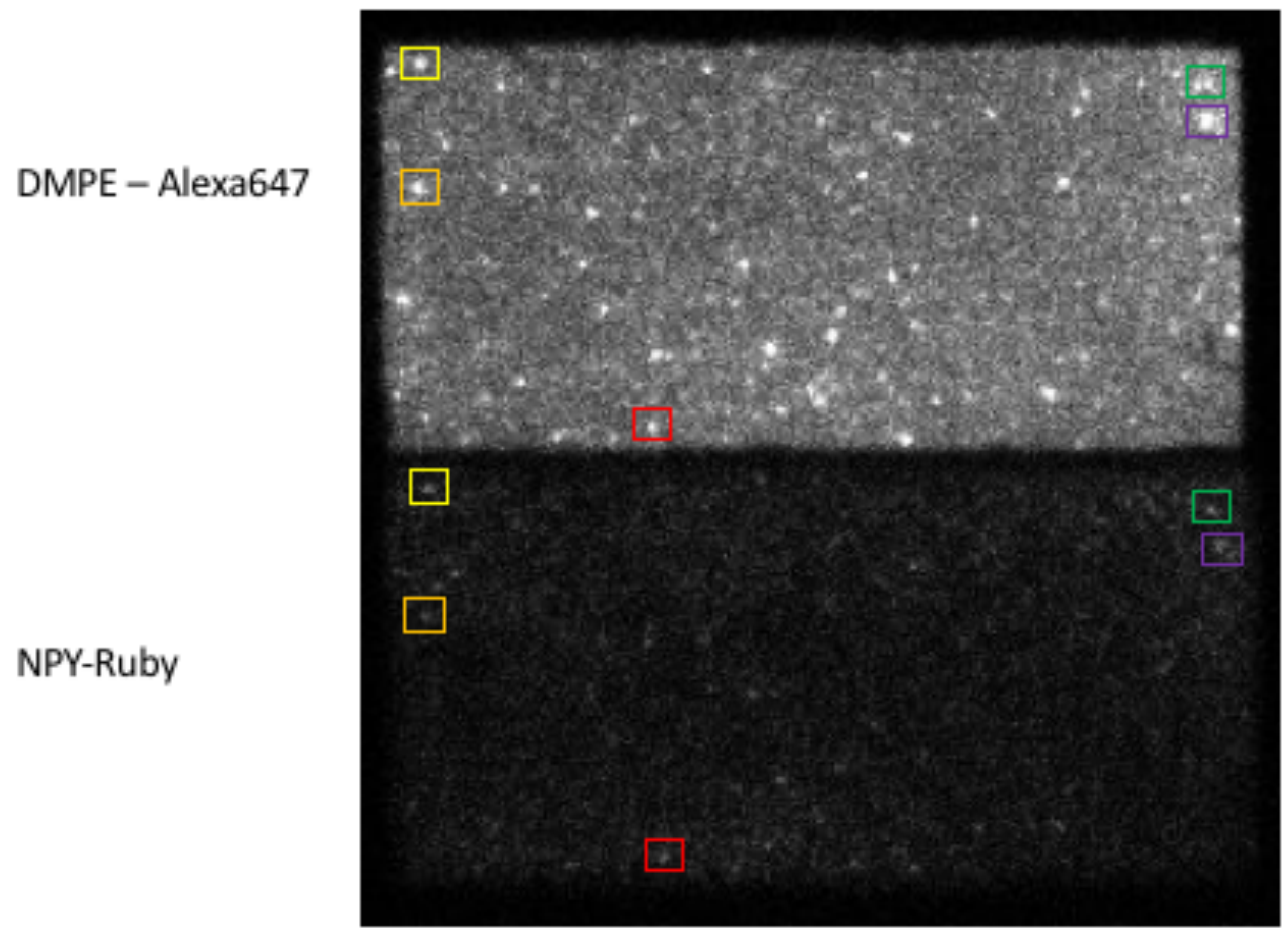

Figure A1.4: View of DCV sample when opti-split is used to separate fluorescence of DMPEAlexa647 from NPY-mCherry. Corresponding fluorescence between channels are shown by colored boxes.
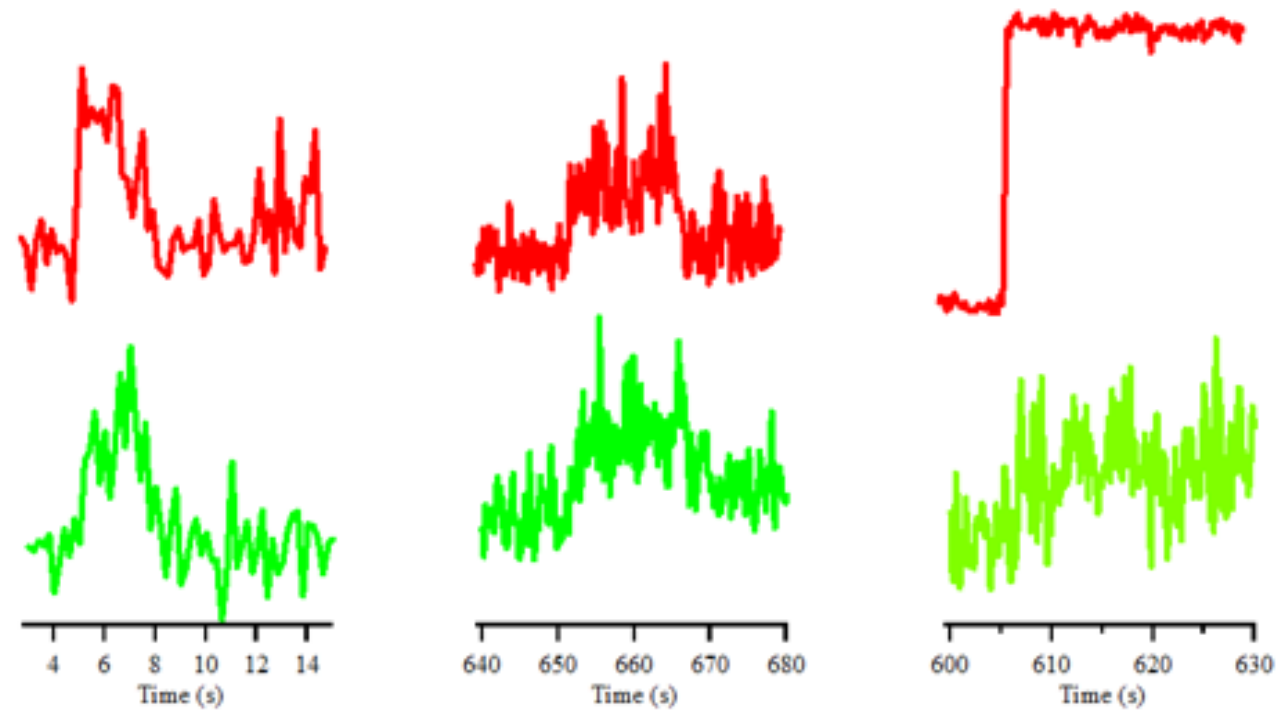

Figure A1.5: Fusion (left and center intensity traces) and docking (right intensity trace) of DCV labeled with DMPE-Alexa647 (red trace) and NPY-Ruby (green trace). 


\section{A2: Asymmetric PE Distribution Controls Fusion Pore Lifetime and Probability}

\section{Introduction}

Phosphatidylethanolamine (PE) is a lipid that has an asymmetric distribution in the plasma membrane and has been proposed to stabilize fusion pores. The role of the asymmetric distribution of PE in effecting SNARE mediated fusion as never been experimentally tested due to the difficulty of preparing asymmetric model membranes. Here, we will use the asymmetric planar supported bilayer that mimic's the plasma membrane of a cell to examine the effect on fusion of PE distribution between leaflets of a lipid bilayer. The fusion of DCVs with the planar supported bilayer was monitored using a fluorescently tagged neuropeptide Y (NPY-Ruby). Four planar supported bilayer conditions were examined being PE $(25 \mathrm{~mol} \%)$ in the outer planar supported bilayer leaflet (plasma membrane mimicking), PE (25 mol\%) in the inner planar supported bilayer leaflet, PE (25 mol\% per leaflet) in both planar supported bilayer leaflets, and planar supported bilayers without PE.

\section{Results}

Phosphatidylethanolamine (PE) lipids have a negative spontaneous curvature, which could stabilize fusion pores (Churchward, et al., 2008), and the plasma membrane has asymmetric lipid distributions where PE is enriched in the cytoplasmic leaflet (Calderon \& DeVries, 1997; van Meer, et al., 2008). This PE distribution could be highly relevant for regulated exocytosis were the asymmetric distribution of PE in the plasma membrane could assist in SNARE mediated 
fusion pore formation. Simulations have predicted that PE could enhance the fusion rate when distributed on the outward facing leaflet of liposomes (Kasson \& Pande, 2007) but a systematic study of PE distribution has not been experimentally tested due to difficulty in preparation of liposomes with asymmetric lipid composition.

The unique system of planar supported bilayer fusion, allows the role of asymmetric PE distribution to be investigated in SNARE mediated membrane fusion. This was done by observing DCV fusion with planar bilayers where the distribution of PE was varied. This was done by having cytosolic mimicking outer leaflet of the planar bilayer but varying the PE (to have $25 \mathrm{~mol} \%$ PE per leaflet) to be in the outer leaflet only, inner leaflet only, in both leaflets, and not present in the planar supported bilayer, Figure A2.1B.

Previously, the diffusion of the neuropeptide fluorophore (NPY-Ruby) was characterized by a decrease of a fluorescent indicating diffusion of the peptide through a fusion pore in the planar supported bilayer, the DCV then collapses into the planar supported bilayer resulting in an increase in fluorescence due to the TIRF field, and then the fluorescence continues to decrease as NPY-Ruby diffuses away from the fusion site, Figure A2.1A (A. J. Kreutzberger, et al. Submitted). The extent and length of the fluorescence decrease before the collapse of the DCV into the TIRF field changes based on the asymmetric distribution of PE, Figure A2.1A. PE in the outer leaflet (cytoplasmic mimicking) results in a substantial decrease in fluorescence prior to pore collapse, while PE in the inner leaflet has almost no decrease within the time resolution of 
the experiment ( $200 \mathrm{~ms})$. The change indicates PE in the outer leaflet stabilizes the DCV/planar supported bilayer fusion pore. Having PE present in both leaflets of the fusion pore results in a small, steep decrease in fusion before DCV collapse and no PE in the planar bilayer results in a slow, more gradual decrease before the collapse as compared to the condition with PE in the outer leaflet. The kinetics of delay times between docking and fusion are shown in Figure A2.1C with a summary of events shown in Table A2.1. PE present in the outer leaflet had the most efficient fusion while PE in the inner leaflet had the least, while PE in both leaflets or no PE present had intermediate fusion efficiencies, Figure A2.1C.

This result shows the advantages of this planar bilayer assay in being able to control the lipid distribution in both leaflets while the NPY-Ruby fluorescence in the DCV allows for a unique readout relating to fusion pore lifetime. The effect of PE asymmetry on DCV fusion implicate the importance PE in the cytoplasmic leaflet of the plasma membrane in stabilizing fusion pore while directly contributing to an efficient fusion reaction. 
A)

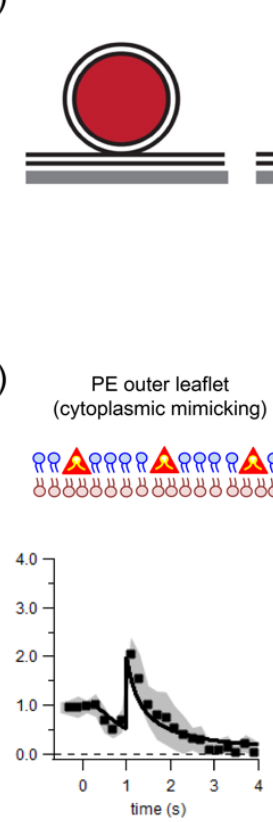

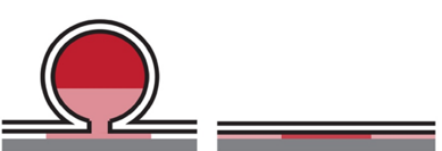

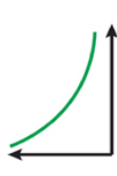

C)

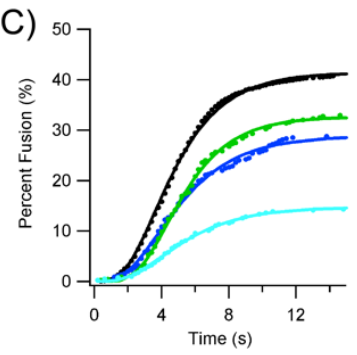

PE both leaflet RRARRRTARRRTART

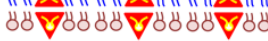

No PE
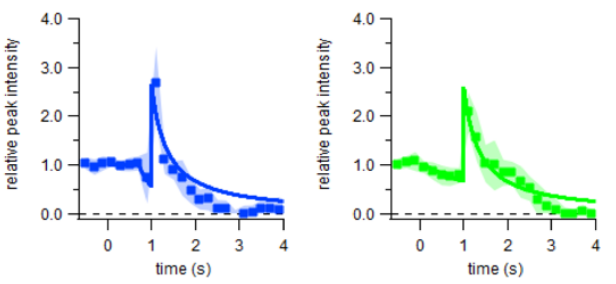

Figure A2.1: A) Two-step model of DCV fusion event in a TIRF field. DCV dock to planar supported bilayers, where a fusion pore opens releasing the fluorescence NPY-Ruby from the DCV, the DCV then collapse into the planar supported bilayer pulling NPY-Ruby forward in the TIRF field which causes an increase in fluorescence as observed in the characteristic intensity traces of DCV fusion events. B) Characteristic fusion intensity traces for different distribution of planar supported bilayer PE. Dots are normalized intensity from 10 averaged fusion events with standard error shown in shaded area. Solid line is a fit of the two-step diffusion model shown in A with pore lifetimes of $0.7 \mathrm{~s}$, $0 \mathrm{~s}, 0.3 \mathrm{~s}$, and $0.9 \mathrm{~s}$ for PE in the outer leaflet, inner leaflet, both leaflets and not present, respectively. The pore lifetime of $0 \mathrm{~s}$ for $\mathrm{PE}$ in the inner leaflet indicates that the pore lifetime is shorter than the frame rate of $200 \mathrm{~ms}$ used in these experiments. C) The kinetics of fusion are shown in a cumulative distribution function of the delay time between DCV docking and pore opening for PE in the outer leaflet (black), PE in the inner leaflet (cyan), PE in both leaflets (blue), and no PE present (green). 


\begin{tabular}{|c|c|c|c|c|c|c|c|c|}
\hline Condition & Inner Leaflet & Outer Leaflet & $\begin{array}{l}\text { Number of } \\
\text { Experiments }\end{array}$ & $\begin{array}{l}\text { Percent } \\
\text { Fusion }\end{array}$ & $\begin{array}{l}\text { Number of } \\
\text { Docking }\end{array}$ & $\begin{array}{l}\text { Number of } \\
\text { Fusion }\end{array}$ & $\mathbf{k}$ & $\mathbf{m}$ \\
\hline PE Outer & $70: 30 \mathrm{bPC}:$ Chol & $\begin{array}{c}\text { 25:25:15:30:4:1 } \\
\text { bPC:bPE:bPS:Chol:PI:PIP2 }\end{array}$ & 14 & $41 \pm 0.9$ & 823 & 340 & $0.44 \pm 0.1$ & $4.8 \pm 0.2$ \\
\hline NoPE & $70: 30 \mathrm{bPC}: \mathrm{Chol}$ & $\begin{array}{c}\text { 50:15:30:4:1 } \\
\text { bPC:bPS:Chol:PI:PIP2 }\end{array}$ & 5 & $33.2 \pm 3.4$ & 0 & 340 & $0.47 \pm 0.04$ & $4.8 \pm 1.1$ \\
\hline PE Inner & $\begin{array}{c}\text { 45:25:30 } \\
\text { bPC:bPE:Chol }\end{array}$ & $\begin{array}{c}\text { 50:15:30:4:1 } \\
\text { bPC:bPS:Chol:PI:PIP2 }\end{array}$ & 5 & $14.6 \pm 1.7$ & 0 & 419 & $0.38 \pm 0.06$ & $64.5 \pm 1.0$ \\
\hline PE Both & $\begin{array}{c}\text { 45:25:30 } \\
\text { bPC:bPE:Chol }\end{array}$ & $\begin{array}{c}\text { 25:25:15:30:4:1 } \\
\text { bPC:bPE:bPS:Chol:PI:PIP2 }\end{array}$ & 5 & $29.0 \pm 3.1$ & 0 & 455 & $0.38 \pm 0.03$ & $34.3 \pm 0.5$ \\
\hline
\end{tabular}

Table A2.2: Summary of statistics for DCV fusion with planar supported bilayer under different PE lipid conditions. All events were fit with a parallel reaction model $\left(N(t)=N\left(1-e^{-k t}\right)^{m}\right.$ where $N$ is the fusion probability, $k$ is the rate, and $m$ is the number of parallel reactions occurring) for the cumulative distribution function of delay times between docking and fusion for single DCV events under different PE lipid conditions. 


\section{A3 Examining fusion pores with electron microscopy}

Fusion intermediates have been observed previously by cryo-EM for SNARE mediated membrane fusion (Hernandez, et al., 2012; Bharat, et al., 2014) and influenza virus membrane fusion (Chlanda, et al., 2016). Here, a description of preliminary attempts to observed membrane fusion will be described.

\section{Preliminary Results}

To observe membrane fusion and to be able to differential between plasma membrane t-SNARE membranes and v-SNARE membranes, t-SNAREs were reconstituted into LUVs $(\sim 100 \mathrm{~nm}$ diameter) and v-SNAREs were reconstituted into SUVs ( $\sim 40 \mathrm{~nm})$. Liposomes were mixed and then rapidly frozen at different time points. Membrane contact sites between t- and v-SNARE membranes were observed, Figure A3.1. Initial these were thought to be possible intermediate stages leading to pore formation and these states were grouped qualitatively into different categories, Figure A3.1 and A3.2. To get more quantitative statistics the width of area of membrane contact was measured as well as the distance from the inner leaflet of each membrane from the center of the contact site, Figure A3.2. To better distinguish when or if pore formation was occurring encapsulation of a heavy metal into one proteoliposome population was desired but has not yet been successfully been accomplished. Tomography was also used to try to reconstruct fusion events in 3-dimensional space with a fusion event shown in Figure A3.3. The initial tomography data revealed different z- planes of the same fusion event would appear like there was no contact site, a fusion pore, or other membrane intermediates, Figure A3.3. This 
indicates the initial EM results in 2 dimensions are not conclusively able to distinguish membrane fusion.

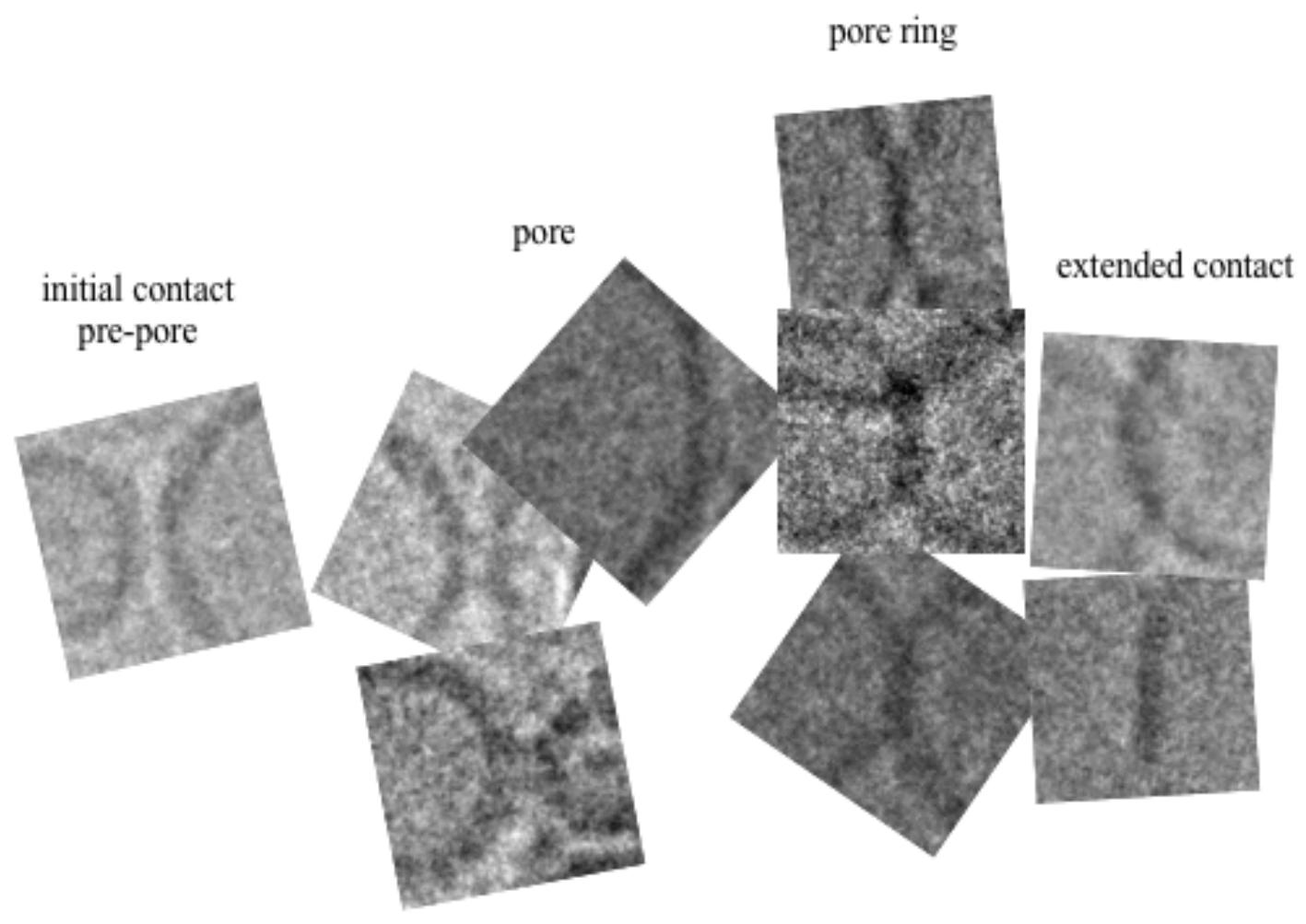

Figure A3.1: Individual vesicle contact sites of proteoliposomes containing the plasma membrane and vesicle SNARE proteins. Images from the left to the right are what is speculated to be snapshots of different intermediates leading to pore progression. The liposomes used in this study were POPC:Chol (80:20) which was shown by TIRF to have very little $(<2 \%$ stable off pathway hemifusion events (Kreutzberger, et al., 2016). 

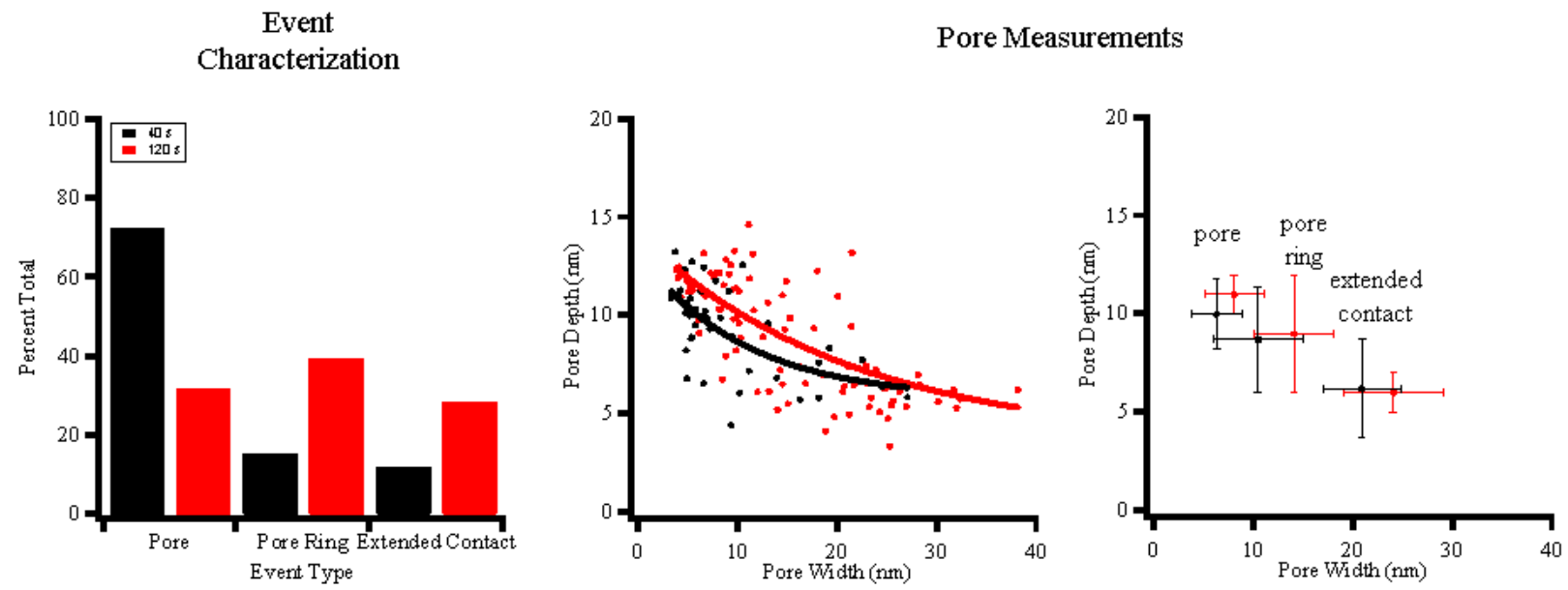

Figure A3.2: Preliminary attempts to quantify pore differences of EM grids frozen after different mixing times of corresponding plasma membrane and v-SNARE proteoliposomes $(40 \mathrm{~s}$ in black and $120 \mathrm{~s}$ in red). Left panel is qualitative classification. Middle and right panel are different plots of the measured length of the liposome contact site and the depth from the inner leaflet of each membrane. 

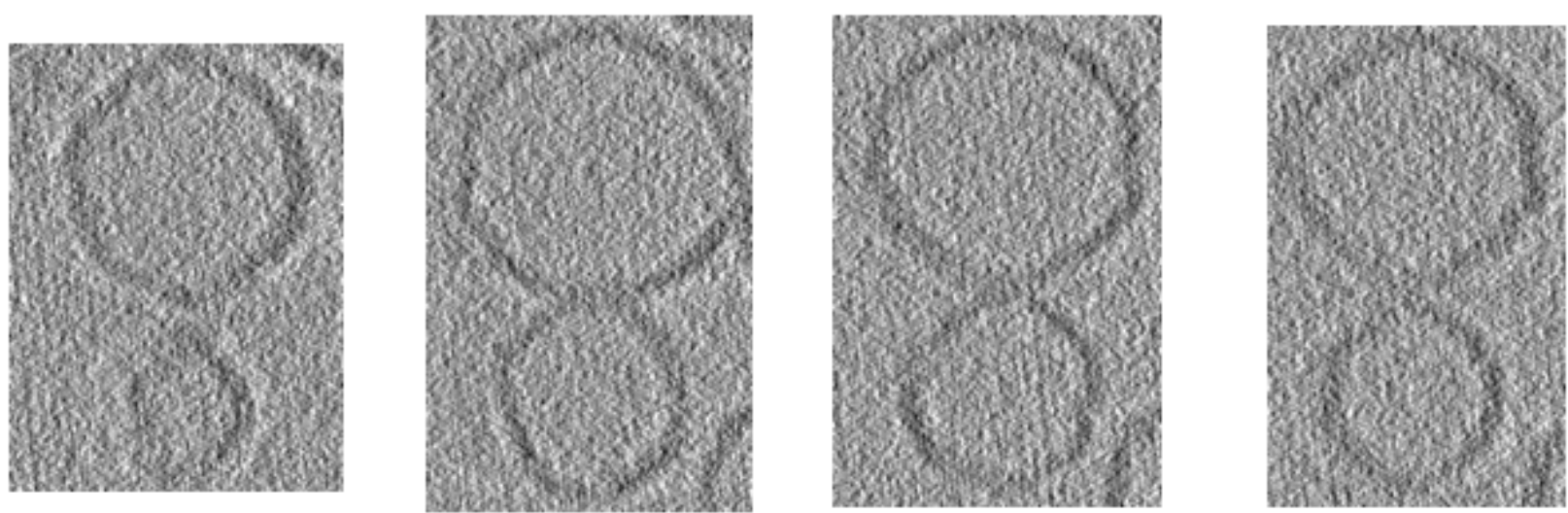

Figure A3.3: A single plasma membrane/v-SNARE fusion event tomogram where it is revealed that the position of the plane of the vesicles effects the observation of membrane contact sites. All four images are the same event put in different $z$ - planes. 


\section{A4: Imaging whole cell exocytosis by fluorescence microscopy}

Coupling results of whole cell exocytosis to planar supported bilayer experiments could add new biologically relevant insights into regulated exocytosis. Several groups observe exocytosis in PC12 cells by labeling soluble DCV proteins (such as NPY) or DCV membrane proteins with fluorescent proteins that have a low fluorescence in the acidic environment of the interior of a DCV but exhibit high fluorescence at neutral $\mathrm{pH}$ which is present when fusion occurs (Taraska, et al., 2003; Rao, et al., 2004). Here, a description of initial experiments of exocytosis of NPY labeled with a $\mathrm{pH}$ sensitive version of GFP (termed pHluorin) will be shown. NPY-pHluorin was transfected into PC12 cells which were the coated onto quartz slides coated in poly-Dlysine. After growing in medium for 3 days the cells were washed with a low potassium buffer (150 mM NaCl, $1.1 \mathrm{mM} \mathrm{KCl,} 1 \mathrm{mM} \mathrm{NaH}_{2} \mathrm{PO}_{2}, 0.7 \mathrm{mM} \mathrm{CaCl}_{2}$, and $10 \mathrm{mM} \mathrm{HEPES}, \mathrm{pH}$ 7.4) and the mounted into a chamber and placed on the TIRF microscopy. Cells were stimulated by replacing the buffer with buffer containing higher amounts of $\mathrm{KCl}$. Characteristic fusion traces of NPY-pHluorin are shown, Figure A4.1. Adding increasing amounts of potassium increased the total number of exocytosis events observed, Figure A4.2. A transient knock-down of synaptotagmin-1 appeared to decrease stimulation of intermediated potassium concentrations, but this result should be repeated Figure A4.2.

Attempts were made to quantify NPY-pHluorin exocytosis. Timing of exocytosis from stimulation was down to make kinetics of events, Figure A4.3. To better quantitatively analyze the type of event the decay of fluorescence signal was fitted with an exponential equation to 
determine rates of decays which were then plotted as histograms for different stimulations of potassium, Figure A4.3.
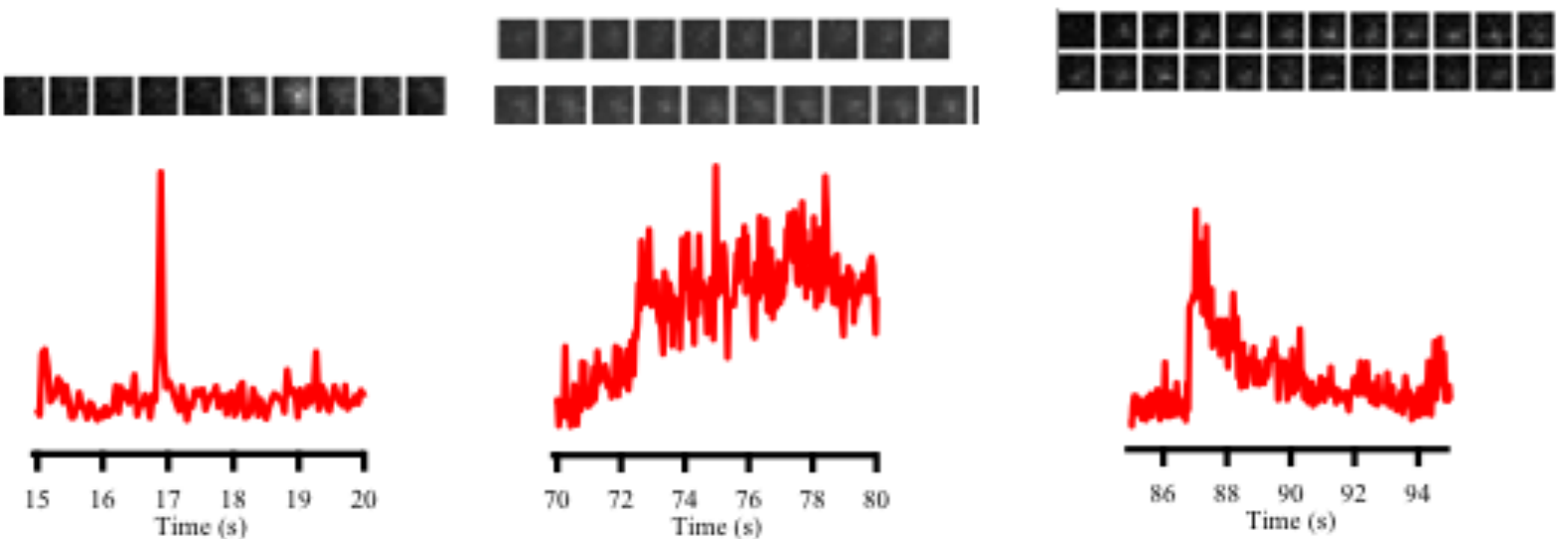

Figure A4.1: Different NPY-pHluorin intensity profiles after the stimulation of fusion. Events of rapid increase in fluorescence that then immediately decreased, events that increased fluorescence then stayed constant, and events where fluorescence increased but then had a slower decrease in fluorescence were observed.

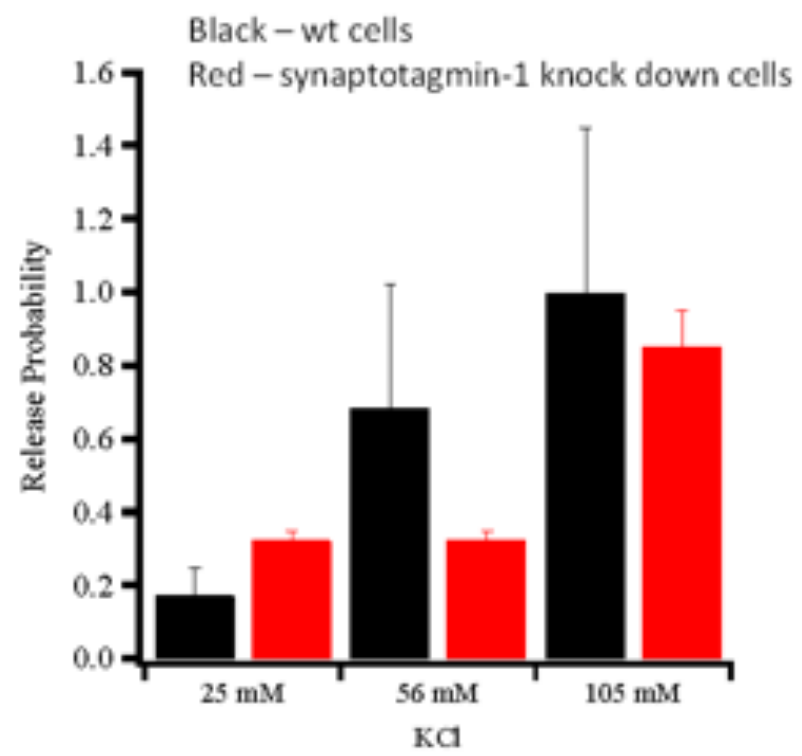

Figure A4.2: Total number of observed NPY-pHluorin appearance events at different concentrations of potassium depolarizations for wild-type PC12 cells and cells that had a transient knockdown of synaptotagmin-1 (done by electroporation). These results come from 5 to 10 cells and need to be repeated. 
$56 \mathrm{mM} \mathrm{KCl}$
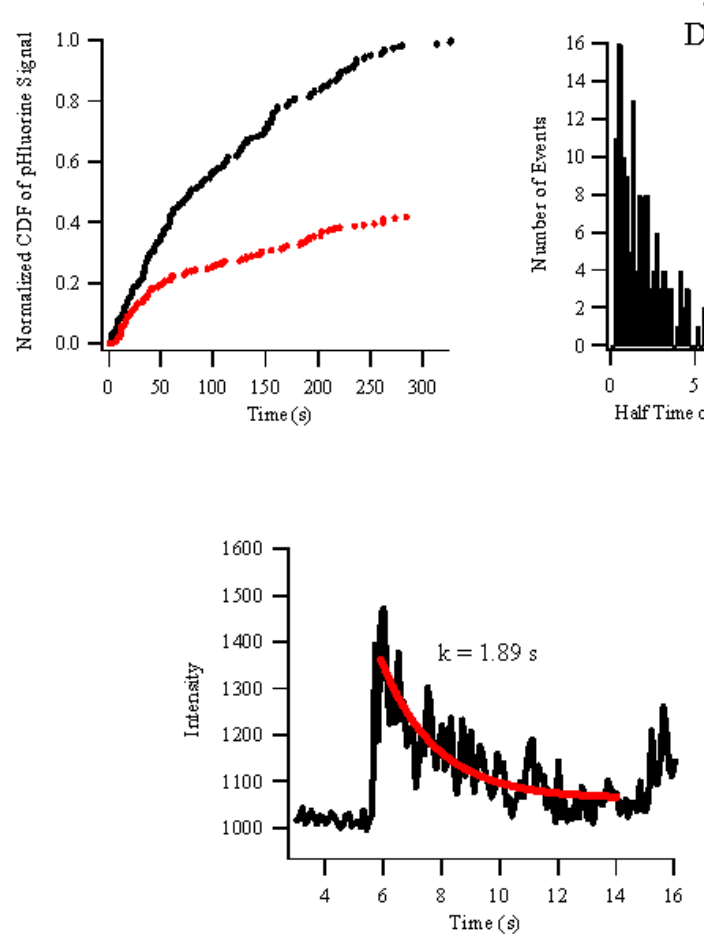

Depolarization

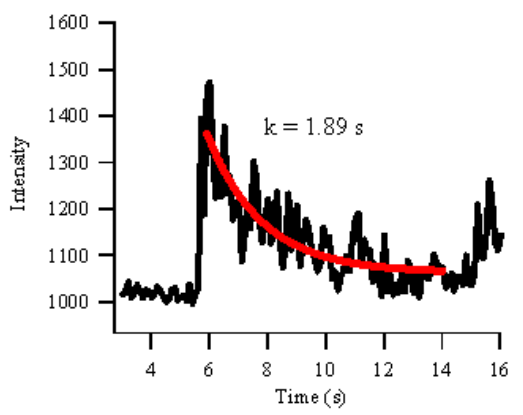

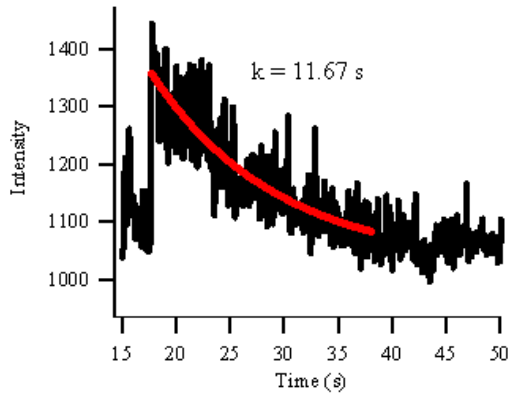

Figure A4.3: Preliminary attempts at quantifying whole cell exocytosis events. Top left panel: is delay times of pHluorin appearance from time of depolarization. Bottom panels are individual events that were fit to determine the decay time of fluorescence intensity decreases. The top middle and right panel show histograms of fluorescence intensity decays. 


\section{References}

Abderrahmani, A., Niederhause, G., Plaisance, V., Roehrch, M.E., Lenain, V., Coppola, T., Regazzi, R., Waeber, G. (2004). Complexin I regulates glucos-induced secretion in pancreatic beta-cells. J Cell Sci 117, 2239-2247.

Aeffner, S., Reusch, T., Weinhausen, B., Salditt, T. (2012). Energetics of stalk intermediates in membrane fusion are controlled by lipid composition. Proc Natl Acad Sci USA 109, 1609-1618.

Albillos, A., Dernick, G., Horstmann, H., Almers, W., Alvarez de Toledo, G., Lindau, M. (1997). The exocytotica event in chromaffin cells revealed by patch amperometry. Nature 389, 509-512.

Altenbach, C., Greenhalgh, D.A., Khorana, G., Hubbell, W.L. (1994). A collision gradient method to determine the immersion depth of nitroxides in lipid bilayers: application to spinlabeledmutants of bacteriorhodopsin. Proc Natl Acad Sci USA 91, 1667-1671.

Ann, K., Kowalchyk, J.A., Loyet, K.M., Martin, T.F. (1997). Novel Ca2+ binding protein (CAPS) related to UNC-31 required for Ca2_activated exocytosis. J Biol Chem 272, 1963719640.

Bacia, K., Schuette, C.G., Kahya, N., Jahn, R., Schwille, P. (2004). SNAREs prefer liquiddisordered over "raft" (liquid ordered) domains when reconstituted into giant unilamellar vesicles. J Biol Chem 279, 37951-37955. 
Bader, M.F., Holz, R.W., Kumakura, K., Vitale, N. (2002). Exocytosis: the chromaffin cell as a model system. Ann N Y Acad Sci 971, 178-183.

Baker, R.W., Jeffrey, P.D., Zick, M., Phillips, B.P., Wickner, W.T., Hughson, F.M. (2015). A direct role for the Sec1/Munc18-family protein Vps33 as a template for SNARE assembly. Science 349, 1111-1114.

Bartlett, G.R. (1959). Phosphorus assay in column chromatography. J Biol Chem 234, 466-468.

Bethani, I., Werner A., Kadian, C., Geumann, U., Jahn R., Rizzoli, S.O. (2009). Endosomal fusion upon SNARE knockdown is maintained by residual SNARE activity and enhanced docking. Traffic 10, 1543-1559.

Bharat, T.A., Malsam, J., Hagen, W.J., Scheutzow, A., Söllner, T.H., Briggs, J.A. (2014).

SNARE and regulatory proteins induce local membrane protrusions to prime docked vesicles for fast calcium-triggered fusion. EMBO Rep 15, 308-314.

Borisovska, M., Zhao, Y., Tsytsyura, Y., Glyvuk, N., Takamori, S., Matti, U., Rettig, J., Südhof, T.C., Bruns, D. (2005). v-SNAREs control exocytosis of vesicles from priming to fusion. EMBO $\mathrm{J} 24,2114-2126$. 
Boswell, K.L., James, D.J., Esquibel, J.M., Bruinsma, S., Shirakawa, R., Horiuchi, H., Martin, T.F. (2012). Munc13-4 reconstitutes calcium-dependent SNARE-mediated membrane fusion. J Cell Biol 197, 301-312.

Bowen, M.E., Weninger, K., Brunger, A.T., Chu, S. (2004). Single molecule observation of liposomes-bilayer fusion thermally induced by soluble N-ethyl maleimide sensitive-factor attachment protein receptors (SNAREs). Biophys J 87, 3569-3584.

Brandford, A., Atkinson, J., Fuller, N., Rand, R.P. (2003). The effect of vitamin E on the structureof membrane lipid assemblies. J Lipid Res 44, 1940-1945.

Brose, N. (2008). For better or for worse: complexins regulate SNARE function and vesicle fusion. Traffic, 9, 1403-1413.

Brose, N., Petrenko, A.G., Südhof, T.C., Jahn, R. (1992). Synaptotagmin: A calcium sensor on the synaptic vesicle surface. Science 256, 1021-1025.

Calderon, R., DeVries, G.H. (1997). Lipid composition and phospholipid asymmetry of membranes from a Schwann cell line. J Neurosci Res 49, 372-380.

Chamberlain, L.H., Burgoyne, R.D., Gould, G.W. (2001). SNARE proteins are highly enriched in lipid rafts in PC12 cells: implications for the spatial control of exocytosis. Proc Natl Acad Sci USA 98, 5619-5624. 
Chang, J., Kim, S.A., Lu, X., Su, Z., Kim, S.K., Shin, Y.K. (2009). Fusion step-specific influence of cholesterol on SNARE-mediated membrane fusion. Biophys J 96, 1839-1846.

Chasserot-Golaz, S., Coorssen, J.R., Meunier, F.A., Vitale, N. (2010). Lipid dynamics in exocytosis. Cell Mol Neurobiol 30, 1335-1342.

Chen, X., Arac, D., Want, T.M., Gilpin, C.J., Zimmerberg, J., Rizo, J. (2006). SNARE-mediated lipid mixing depends on the physical state of the vesicles. Biophys J 90, 2062-2074.

Chen, X., Tomchick, D.R., Kovrigin, E., Arac, D., Machius, M., Südhof, T.C., Rizo, J. (2002). Three-dimensional structure of the complexin/SNARE complex. Neuron 33, 397-409.

Chen, Z., Rand, R.P. (1997). The influence of cholesterol on phospholipid membrane curvature and bending elasticity. Biophys J 73, 267-276.

Chernomordik, L.V., Kozlov, M.M. (2005). Membrane hemifusion: crossing a chasm in two leaps. Cell 123, 375-382.

Chernomordik, L.V., Kozlov, M.M. (2008). Mechanics of membrane fusion. Nat Struct Mol Biol $15,675-683$.

Chlanda, P., Mekhedov, E., Waters, H., Schwartz, C.L., Fischer, E.R., Ryham, R.J., Cohen, F.S., Blank, P.S., Zimmerberg, J. (2016). The hemifusion structure induced by influenza virus 
haemagglutinin is determined by physical properties of the target membranes. Nat Microbiol 1, 16050.

Choi, U.B., Zhao, M., Zhang, Y., Lai, Y., Brunger, A.T. (2016). Complexin induces a conformational change at the membrane-proximal C-terminal end of the SNARE complex. Elife $5, \mathrm{e} 16886$.

Chung, S.H., Song, W.J., Kim, K., Bednarski, J.J., Chen, J., Prestwich, G.D., Holz, R.W. (1998). The C2 domains of Rabphilin3A specifically bind phosphatidylinositol 4,5-bisphosphate containing vesicles in a $\mathrm{Ca} 2+$ dependent manner in vitro characteristics and possible significance. J Biol Chem 273, 10240-10248.

Churchward, M.A., Coorssen, J.R. (2009). Cholesterol, regulated exocytosis and the physiological fusion machine. Biochem J 413, 1-14.

Churchward, M.A., Rogasevskaia, T., Brandman, D.M., Khosravani, H., Nava, P., Atkinson, J.K., Coorssen, J.R. (2008). Specific lipids supply critical negative spontaneous curvature -- an essential component of native Ca2+-triggered membrane fusion. Biophys J 94, 3976-3986.

Churchward, M.A., Rogasevskaia, T., Höfgen, J., Bau, J., Coorssen, J.R. (2005) Cholesterol facilitates the native mechanism of Ca2+-triggered membrane fusion. J Cell Sci 118, 4833-4848. 
Columbus, L., Hubbel, W.L. (2002). A new spin on protein dynamics. Trends Biochem Sci 27, 288-295.

Columbus, L., Kalai, T., Jakö, J., Hideg, K., Hubbell W.L. (2001). Molecular motion of spin labeled side chains in $\alpha$-helices: analysis by variation of sidechain structure. Biochem 40, 32283846.

Coorssen, J.R., Rand, R.P. (1990). Effects of cholesterol on the structural transitions induced by diacylglycerol in phosphatidylcholine and phosphatidylethanolamine bilayer systems. Biochem Cell Biol, 68, 65-69.

Crane, J.M., Kiessling, V., Tamm, L.K. (2005). Measuring lipid asymmetry in planar supported bilayers by fluorescence interference contrast microscopy. Langmuir 21, 1377-1388.

Crawford, D.C. Kavalali, E.T. (2015). Molecular underpinnings of synaptic vesicle pool heterogeneity. Traffic 16, 338-364.

Cypionka, A., Stein, A., Hernandez, J.M., Hippchen, H., Jahn, R., Walla, P.J. (2009).

Discrimination between docking and fusion of liposomes reconstituted with neuronal SNAREproteins using FCS. Proc Natl Acad Sci USA 106, 18575-18580. 
Dean, P.M., Matthews, E.K. (1968). Electrical activity in pancreatic islet cells. Nature 290, 389390.

Delaglio, F., Grzesiek, S., Vuister, G.W., Zhu, G., Pfeifer, J., Bax, A. (1995). NMRPipe: a multidimensional spectral processing system based on UNIX pipes. J Biomol NMR 6, 277-293.

Deutsch, J.W., Kelly, R.B. (1981). Lipids of synaptic vesicles: relevance to the mechanism of membrane fusion. Biochem 20, 378-385.

Diao, J., Cipriano, D.J., Zhao, M., Zhang, Y., Shah, S., Padolina, M.S., Pfuetzner, R.A., Brunger, A.T. (2013). Complexin-1 enhances the on-rate of vesicle docking via simultaneous SNARE and membrane interactions. J Am Chem Soc 135, 15274-15277.

Diao, J., Grob, P., Cipriano, D.J., Kyoung, M., Zhang, Y., Shah, S., Nguyen, A., Padolina, M., Srivastava, A., Vrljic, M., Shah, A., Nogates, E., Chu, S., Brunger, A.T. (2012). Synaptic proteins promote calcium-triggered fast transition from point contact to full fusion. eLife 1, e0009.

Domanska, M.K., Kiessling, V., Stein, A., Fasshauer, D., Tamm, L.K. (2009). Single vesicle millisecond fusion kinetics reveals number of SNARE complexes optimal for fast SNAREmediated membrane fusion. J Biol Chem 284, 32158-32166. 
Domanska, M.K., Kiessling, V., Tamm, L.K. (2010). Docking and fast fusion of synaptobrevin vesicles depends on the lipid compositions of the vesicle and the aceptor SNARE complexcontaining target membrane. Biophys J 99, 2936-2946.

Dulubova, I., Sugita, A., Hill, S., Hosaka, M., Fernandez, I., Südhof, T.C., Rizo, J. (1999). A conformational switch in syntaxin during exocytosis: role of Munc18. EMBO J 18, 4372-4382.

Evenas, J., Tugarinov, V., Skrynnikova, N.R., Goto, N.K., Muhandiram, R., Kay, L.E. (2001). Ligand-induced structural changes tomaltodextrin-binding protein as studied by solution NMR spectroscopy. J Mol Biol 309, 961-974.

Fasshauer, D. (2003). Structural insights into the SNARE mechanism. Biochim Biophys Acta $1641,87-97$.

Fasshauer, D., Antonin, W., Subramaniam, V., Jahn, R. (2002). SNARE assembly and disasembly exhibit a pronounced hysteresis. Nat Struct Biol 9, 144-151.

Fasshauer, D., Margittai, M. (2004). A transient N-terminal interaction of SNAP-25 and syntaxin nucleates SNARE assembly. J Biol Chem 279, 7613-7621.

Fasshauer, D., Otto, H., Eliason, W.K., Jahn, R., Brünger, A.T. (1997). Structural changes are associated with soluble $\mathrm{N}$-ethylmaleimide-sensitive fusion protein attachment protein receptor complex formation. J Biol Chem 272, 28036-28041. 
Fasshauer, D., Sutton, R.B., Brunger, A.T., Jahn, R. (1998). Conserved structural features of the synaptic fusion complex: SNARE proteins reclassified as Q- and R-SNAREs. Proc Natl Acad Sci USA 95, 15781-15786.

Fernandez, I., Dulubova, I., Zhang, X., Südhof, T.C., Rizo, J. (1998). Three-dimensional structure of an evolutionarily conserved N-terminal domain of syntaxin 1A. Cell 94, 841-849.

Fix, M., Melia, T.J., Jaiswal, J.K., Rappoport, J.Z., You, D., Söllner, T.H., Rothman, J.E., Simon, S.M. (2004). Imaging single membrane fusion events mediated by SNARE proteins. Proc Natl Acad Sci USA 101, 7311-7316.

Foley, T.D., Clark, A.R., Stredny, E.S. Wierbowski, B.M. (2012). SNAP-25 contains nonacylated thiol pairs that can form intra chain disulfide bonds: possible sites for redox modulation of neurotransmission. Cell Mol Neurobiol 32, 201-208.

Frazier, A.A., Malmberg, N.J., Victor, K.G., Fanucci, G.E., Nalefski, E.A., Falke, J.J., Cafiso, D.S. (2002). Membrane orientation and position of the C2 domain from cPLA2 by site-directed spin labeling. Biochem 41, 6282-6292.

Freed, D.M., Khan, A.K, Horanyi, P.S. Cafiso, D.S. (2011). Molecular origin of electron paramagnetic resonance line shapes on beta-barrel membrane proteins: the local solvation environment modulates spin-label configuration. Biochem 50, 8792-8803. 
Fromherz, P., Kiessling, V., Kottig, K., Zeck, G. (1999). Membranetransistor with giant lipidvesicles touching a silicon chip. App Phys A 69, 571-576.

Gao, Y., Zorman, S., Gundersen, G., Xi, Z., Ma, L., Sirinakis, G., Rothman, J.E., Zhang, Y. (2012). Single reconstituted neuronal SNARE complexes zipper in three distinct stages. Science $337,1340-1343$.

Gendron, P.O., Avaltroni, F., Wilkinson, K.J. (2008). Diffusion coefficients of several rhodamine derivatives as determined by pulsed field gradient-nuclear magnetic resonance and fluorescence correlation spectroscopy. J Fluoresc 18, 1093-1101.

Gerber, S.H., Rah, J.C., Min, S.W., Liu, X., de Wit, H., Dulubova, I., Meyer, A.C., Rizo, J., Arancillo, M., Hammer, R.E., Verhage, M., Rosenmund, C., Südhof, T.C. (2008).

Conformational switch of syntaxin-1 controls synaptic vesicle fusion. Science 321, 1507-1510.

Gil, C., Soler-Jover, A., Blasi, J., Aguilera, J. (2005). Synaptic proteins and SNARE complexes are localized in lipid rafts from rat brain synaptosomes. Biochem Biophys Res Commun 329, $117-124$.

Giraudo, C.G., Eng, W.S., Melia, T.J., Rothman, J.E. (2006). A clamping mechanism involved in SNARE-dependent exocytosis. Science 313, 676-680. 
Giraudo, C.G., Garcia-Diaz, A., Eng, W.S., Chen, Y., Hendrickson, W.A., Melia, T.J., Rothman, J.E. (2009). Alternative zippering as an of-off switch for SNARE-mediated fusion. Science 323, $512-516$.

Goddard, T.D., Kneller, D.G. (n.d.). SPARKY 3. University of California, San Fransisco. https://www.cgl.ucsf.edu/home/sparky/.

Gomi, H., Mizutani, S., Kasai, K., Itohara, S., Izumi, T. (2005). Granuphilin molecularly docks insulin granules to the fusion machinery. J Cell Biol 171, 99-109.

Gondre-Lewis, M.C., Park, J.J., Loh, Y.P. (2012). Cellular mechanisms for the biogenesis and transport of synaptic and dense-core vesicles. Int Rev Cell Bol Biol. 299, 27-115.

Gong, J., Lai, Y., Li, X., Wang, M., Leitz, J., Hu, Y., Zhang, Y., Choi, U.B., Cipriano, D., Pfuetzner, R.A., Südhof, T.C., Yang, X., Brunger, A.T., Diao, J. (C-terminal domain of mammalian complexin-1 localizes to highly curved membranes. Proc Natl Acad Sci USA 113, 7590-7599.

Greenwood, A.I., Tristram-Nagle, S., Nagle, J.F. (2006). Partial molecular volumes of lipids and cholesterol. Chem Phys Lipids 143, 1-10. 
Grishanin, R.N., Kowalchyk, J.A., Klenchin, V.A., Ann, K., Earles, C.A., Chapman, E.R., Gerona, R.R., Martin, T.F. (2004). CAPS acts at a prefusion step in dense-core vesicle exocytosis as a PIP2 binding protein. Neuron 43, 551-562.

Gustavsson, N. Wu, B., Han, W. (2012). Calcium sensing in exocytosis. Adv Exp Med Biol 740, 731-757.

Hagn, F., Etzkorn, M., Raschle, T., Wagner, G. (2013). Optimized phospholipid bilayer nanodiscs facilitate high-resolution structure determination of membrane proteins. J Am Chem Soc $135,1919-1925$.

Hanson, P.I., Heuser, J.E. Jahn, R. (1997). Neurotransmitter release - four years of SNARE complexes. Curr Opin Neurobiol 7, 305-310.

Hao, M., Bogan, J.S. (2009). Cholesterol regulates glucose-stimulated insulin secretion through phosphatidylinositol 4,5-bisphosphate. J Biol Chem 284, 29489-29498.

Hay, J.C., Martin, T.F. (1992). Resolution of regulated secretion into sequential MGATPdependent and calcium-dependent stages mediated by distinct cytosolic proteins. J Cell Biol 119, $139-151$. 
Hernandez, J.M, Kreutzberger, A.J., Kiessling, V., Tamm, L.K., Jahn, R. (2014). Variable cooperativity in SNARE-mediated membrane fusion. Proc NatlAcad Sci USA 111, 1203712042.

Hernandez, J.M., Stein A., Behrmann, E., Riedel, D., Cypionka, A.,Farsi, Z., Walla, P.J., Raunser, S., Jahn, R. (2012). Membrane fusion intermediates via directional and full assembly of the SNARE complex. Science 336, 1581-1584.

Hinterdorfer, P., Baber, G., Tamm, L.K. (1994). Reconstitution of membrane fusion sites. A total internal reflection fluorescence microsocpy study of influenza hemagglutinin-mediated membrane fusion. J Biol Chem. 269, 20360-20368.

Hodgkin, A.L., Huxley, A.F., Katz, B. (1952). Measurement of current-voltage relations in the membrane of the giant axon of Loligo. J physiol 116, 424-448.

Holt, M., Riedel, D., Stein, A., Schuette, C., Jahn, R. (2008). Synaptic vesicles are constitutively active fusion machines that function independently of Ca2+. Curr Biol 18, 715-722.

Holz, R.W., Axelrod, D. (2002). Localization of phosphatidylinositol 4,5-P(2) important in exocytosis and a quantitative analysis of chromaffin granule motion adjacent to the plasma membrane. Ann NY Acad Sci 971, 232-243. 
Honigmann, A., van den Bogaart, G., Iraheta, E., Risselada, H.J., Milovanovic, D., Mueller,V., Müllar, S., Diederichsen, U., Fasshauer, D., Grubmüller, H., Hell, S.W., Eggeling, C., Kühnel, K., Jahn, R. (2013). Phosphatidylinositiol 4,5-bisphosphate clusters act as molecular beacons for vesicle recruitment. Nat Struct Mol Biol 20, 679-686.

Hua, Y., Scheller, R.H. (2001). Three SNARE complexes cooperate to mediate membrane fusion. Proc Natl Acad Sci USA 98, 8065-8070.

Huntwork, S., Littleton, J.T. (2007). A complexin fusion clamp regulates spontaneous neurotransmitter release and synaptic growth. Nature neuroscience 10, 1235-1237.

Huttner, W.B., Schmidt, A. (2000). Lipids, lipid modification, and lipid-protein interaction in membrane budding and fission- insights from the roles of endophilin A1 and synaptophysin in synaptic vesicle endocytosis. Curr Opin Neurobiol 10, 543-551.

Ishizuka, T., Saisu, H., Odani, S., Abe, T. (1995). Synaphin: a protein associated with the docking/fusion complex in presynaptic terminals. Biochem Biophys Res Commun 213, 11071114.

Itakura, M., Misawa, H., Sekiguchi, M., Takahashi, S., Takahashi, M. (1999). Transfection analysis of functional roles of complexin I and II in the exocytosis of two different types of secretory vesicles. Biochem and Biophys Res Commun 265, 691-696. 
Jahn, R., Fasshauer, D. (2012). Molecular machines governing exocytosis of synaptic vesicles. Nature 490, 201-207.

Jahn, R., Scheller, R.H. (2006). SNAREs--engines for membrane fusion. Natures Reviews 7, $631-643$.

James, D.J., Khodthong, C., Kowalchyk, J.A., Martin, T.F. (2008). Phosphatidylinositol 4,5bisphosphate regulates SNARE-dependent membrane fusion. J Cell Biol 182, 355-366.

James, D.J. Martin, T.F. (2013). CAPS and Munc13: CATCHRs that SNARE vesicles. Front Endocrinol 4, 187.

Ji, H., Coleman, J., Yang, R., Melia, T.J., Rothman, J.E., Tareste, D. (2010). Protein determinants of SNARE-mediated lipid mixing. Biophys J 99, 553-560.

Kümmel, D., Krishnakumar, S.S., Radoff, D.T., Li, F., Giraudo, C.G., Pincet, F., Rothman, J.E., Reinisch, K.M. (2011). Complexin cross-links prefusion SNAREs into a zigzag array. Nat Struct Mol Biol 18, 927-933.

Kabachinski, G., Kielar-Grevstad, D.M., Zhang, X., James, D.J., Martin, T.F. (2016) Resident CAPS on dense-core vesicles docks and primes vesicles for fusion. Mol Biol Cell 27, 654-668. 
Kaeser-Woo, Y.J., Yang, X., Südhof, T.C. (2012). C-terminal complexin sequence is selectively required for clamping and priming but not for $\mathrm{Ca} 2+$ triggering of synaptic exocytosis. J Neurosci $32,2877-2885$.

Kalb, E., Frey, S., Tamm, L.K. (1992). Formation of supported planar bilayers by fusion of vesicles to supported phospholipid monolayers. Biochim Biophys Acta 1103, 307-316.

Karatekin, E., Di Giovanni, J., Iborra, C., Coleman, J., O'Shaughnessy B., Seagar, M., Rothman, J.E. (2010). A fast, single-vesicle fusion assay mimics physiological SNARE requirements. Proc Natl Acad Sci USA 107, 3517-3521.

Karatekin, E., Tran, V.S., Huet, S., Fanget, I., Cribier, S., Henry, J.P. (2008). A 20-nm step toward the cell membrane preceding exocytosis may correspond to docking of tethered granules. Biophys J 94, 2891-2905.

Kasson, P.M., Pande, V.S. (2007). Control of membrane fusion mechanism by lipid composition: predictions from ensemble molecular dynamics. PLoS Comput Biol. 3

Kato, N., Nakanishi, M., Hirashima, N. (2003). Cholesterol depletion inhibits store-operated calcium currents and exocytotic membrane fusion in RBL-2H3 cells. Biochem 42, 11808-11814.

Katz, A.M., Messineo, F.C., Herbette, L. (1982). Ion channels in membranes. Circulation 65, 2 10. 
Kiessling, V., Ahmed, S., Domanska, M.K., Holt, M.G., Jahn, R., Tamm, L.K. (2013). Rapid fusion of synaptic vesicles with reconstituted target SNARE membranes. Biophys J 104, 19501958.

Kiessling, V., Bernt, M., Fromherz, P. (2000). Extracellular resistance in cell adhesion measured with a transistor probe.Langmuir16, 3516-3521.

Kiessling, V., Crane, J.M., Tamm, L.K. (2006) Transbilayer effects of raft-like lipiddomains in asymmetric planar bilayers measured by single molecular tracking. Biophys J 91, 3313-3326.

Kiessling, V., Tamm, L.K. (2003). Measuring distances in supported bilayers by fluorescence interference-contrast microscopy: Polymer supports and SNARE proteins. Biophys J 84, 408418.

Kim, J., Zhu, Y., Shin, Y.K. (2016). Preincubation of t-SNAREs with complexin I increases content-mixing efficiency. Biochem 55, 3667-3673.

Kloepper, T.H., Kienle, C.N., Fasshauer, D. (2007). An elaborate classification of SNARE proteins sheds light on the conservation of the eukaryotic endomembrane system. Mol Biol Cell $18,3463-3471$. 
Koseoglu, S., Love, S.A., Haynes, C.L. (2011). Cholesterol effects on vesicle pools in chromaffin cells revealed by carbon-fiber microelectrode amperometry. Anal Bioanal Chem 400, 2963-2971.

Kreutzberger, A.J., Kiessling, V., Liang, B., Seelheim, P., Jakhanwal, S., Jahn, R., Castle, J.D., Tamm, L.K. (Submitted). Reconstitution of calcium-mediated exocytosis of dense core vesicles.

Kreutzberger, A.J., Kiessling, V., Tamm, L.K. (2015). High cholesterol obviates a prolonged hemifusion intermediate in fast SNARE-mediated membrane fusion. Biophys J 109, 319-329.

Kreutzberger, A.J., Liang, B., Kiessling, V., Tamm, L.K. (2016). Assembly and comparision of plasma membrane SNARE acceptor complexes. Biophys J 110, 2147-2150.

Krishnakumar, S.S., Li, F., Coleman, J., Schauder, C.M., Kümmel, D., Pincet, F., Rothman, J.E., Reinisch, K.M. (2015). Re-visiting thetrans insertion model for complexin clamping. Elife 4.

Kronchke, B.M., Horanyi, P.S., Columbus, L. (2010). Structural origins of nitroxide side chain dynamics on membrane protein alpha-helical sites. Biochem 49, 10045-10060.

Kyoung, M., Srivastava, A., Zhang, Y., Diao, J., Vrljic, M., Grob, P., Nogales, E., Chu, S., Brunger, A.T. (2011). In vitro system capable of differentiating fast Ca2+-triggered content mixing from lipid exchange for mechanistic studies of neurotransmitter release. Proc NatlAcad Sci USA 108, 304-313. 
Lai, Y., Choi, U.B., Zhang, Y., Zhao, M., Pfuetzner, R.A., Wang, A.L., Diao, J., Brunger, A.T. (2016). N-terminal domain of complexin independently activates calcium triggered fusion. Proc Natl Acad Sci USA 113, 4698-4707.

Lai, Y., Diao, J., Cipriano, D.J., Zhang, Y., Pfuetzner, R.A., Padolina, M.S., Brunger, A.T. (2014). Complexin inhibits spontaneous release and synchronizes Ca2+-triggered synaptic vesicle fusion by distinct mechanisms. Elife 3.

Lai, Y., Diao, J., Liu, Y., Ishitsuka, Y., Su, Z., Schulten, K., Ha, T., Shin, Y.K. (2013). Fusion pore formation and expansion induced by $\mathrm{Ca} 2+$ and synaptotagmin 1 . Proc Natl Acad Sci USA 110, 1333-1338.

Lang, T. (2007). SNARE proteins and 'membrane rafts'. J Physiol 585, 693-698.

Lang, T., Bruns, D., Wenzel, D., Riedel, D., Holroyd, P., Thiele, C., Jahn, R. (2001). SNAREs are concentrated in cholesterol-dependent clusters that define docking and fusion sites for exocytosis. EMBO J 20, 2202-2213.

Lerman, J.C., Robblee, J., Fairman, R., Hughson, F.M. (2000). Structural analysis of the neuronal SNARE protein syntaxin-1A. Biochem 39, 8470-8479. 
Liang, B., Dawidowski, D., Ellena, J.F., Tamm, L.K., Cafiso, D.S. (2014). The SNARE motif of synaptobrevin exhibits an aqueous-interfacial partitioning that is modulated by membrane curvature. Biochem 53, 1485-1494.

Liang, B., Kiessling, V., Tamm, L.K. (2013). Prefusio structure of syntaxin-1A suggests pathway for folding into neuronal trans-SNARE complex fusion intermediate. Proc Natl Acad Sci USA 110, 19384-19389.

Li, F., Pincet, F., Perez, E., Eng, W.S., Melia, T.J., Rothman, J.E., Tareste, D. (Energetics and dynamics of SNAREpin folding across lipid bilayers. Nat Struct MolBiol 14, 890-896.

Linetti, A., Fratangeli, A., Taverna, E., Valnegri, P., Francolini, M., Cappello, V., Matteoli, M., Passafaro, M., Rosa, P. (2010). Cholesterol reduction impairs exocytosis of synaptic vesicles. J Cell Sci 123, 595-605.

Lingwood, D., Simons, K. (2010). Lipid rafts as a membrane organizing principle. Science 327 , 46-50.

Lv, J.H., He, L., Sui, S.F. (2008). Lipid rafts association of synaptotagmin I on synaptic vesicles. Biochem 73, 283-288.

Lynch, K.L., Martin, T.F. (2007). Synaptotagmins I and IX function redundantly in regulated exocytosis but not endocytosis in PC12 cells. J Cell Sci 120, 617-627. 
Ma, C., Su, L., Seven, A.B., Xu, Y., Rizo, J. (2013). Reconstitution of the vital functions of Munc18 and Munc13 in neurotransmitter release. Science 339, 421-425.

Malsam, J., Parisotto, D., Bharat, T.A., Scheutzow, A., Krause, J.M., Briggs, J.A., Söllner, T.H. (2012). Complexin arrests a pool of docked vesicles for fast Ca2+-dependent release. EMBO J $31,3270-3281$.

Martens, S., McMahon, T.H. (2008). Mechanisms of membrane fusion: Disparate players and common principles. Nat Rev Mol Cell Biol 9, 543-556.

Martin, T.F. (2003). Tuning exocytosis for speed: fast and slow modes. Biochim Biophys Acta $1641,157-165$.

Martin, T.F. (2012). Role of PI(4,5)P(2) in vesicle exocytosis and membrane fusion. Biochem $59,111-130$.

Milosevic, I., Sørensen, J.B., Lang, T., Krauss, M., Nagy, G., Haucke, V., Jahn, R., Neher, E. (2005). Plasmalemmal phosphatidylinositol-4,5-bisphosphate level regulates the releasable vesicle pool size in chromaffin cells. J Neurosci 25, 2557-2565.

Misura, K.M.,Scheller,R.H., Weis, W.I. (2000). Three-dimensional structure of the neuronalSec1-syntaxin-1a complex. Nature 404, 355-362. 
Mitter, D., Reisinger C, Hinz B, Hollmann S, Yelamanchili SV, Treiber-Held S, Ohm TG, Herrmann A, Ahnert-Hilger G. (2003). The synaptophysin/synaptobrevin interaction critically depends on the cholesterol content. J Neurochem 84, 35-42.

Mohrmann, R., de Wit H, Verhage M, Neher E, Sørensen JB. (2010). Fast vesicle fusion in living cells requires at least three SNARE complexes. Science 330, 502-505.

Montecucco, C., Schiavo, G., Pantano, S. (2005). SNARE complexes and neuroexocytosis: How many, how close? Trends Biochem Sci 30, 367-372.

Murray, D.H., Tamm, L.K. (2009). Clustering of syntaxin-1a in model membranes is modulated by phosphatidylinositol 4,5-bisphosphate and cholesterol. Biochem 48, 4617-4615.

Murray, D.H., Tamm, L.K. (2011). Molecular mechanism of cholesterol- and polyphosphoinositide mediated syntaxin clustering. Biochem 50, 9014-9022.

Neher, E., Marty, A. (1982). Discrete changes of cell membrane capacitance observed under conditions of enhanced secretion in bovine chromaffin cells. Proc Natl Acad Sci USA 79, 67126716.

Nguyen Truong, C.Q., Nestvogel, D., Ratai, O., Schirra, C., Stevens, D.R., Brose, N., Rhee, J., Rettig, J. (2014). Secretory vesicle priming by CAPS isindependentof its SNARE-binding MUN domain. Cell Rep 9, 902-909. 
Pabst, S., Hazzard, J.W., Antonin, W., Südhof, T.C., Jahn, R., Rizo, J., Fasshauer, D. (2000). Selective interaction of complexin with the neuronal SNARE complex. J Biol Chem 275, 1980819818.

Pan, J., Mills, T.T., Tristram-Nagle, S., Nagle, J.F. (2008). Cholesterol perturbs lipid bilayers nonuniversally. Phys Rev Lett 100, 1981103.

Park, Y., Hernandez, J.M., van den Bogaart, G., Ahmed, S., Holt, M., Riedel, D., Jahn, R. (2012). Controlling synaptotagmin activity by electrostatic screening. Nat Struct Mol Biol 19, 991-997.

Park, Y., Seo, J.B., Fraind, A., Pérez-Lara, A., Yavuz, H., Han, K., Jung, S.R., Kattan, I., Walla, P.J., Choi, M., Cafiso, D.S., Koh, D.S., Jahn, R. (2015). Synaptotagmin-1 binds to PIP(2)containing membrane but not to SNAREs at physiological ionic strength. Nat Struct Mol Biol 22, $815-823$.

Parlati, F., Weber, T., McNew, J.A., Westermann, B., Söllner, T.H., Rothman, J.E. (1999). Rapid and efficient fusion of phospholipid vesicles by the alpha-helical core of a SNARE complex in the absence of an N-terminal regulatory domain. Proc Natl Acad Sci USA 96, 12565-12570.

Pobbati, A.V., Stein, A., Fasshauer, D. (2006). N- to C-terminal SNARE complex assembly promotes rapid membrane fusion. Science 313, 673-676. 
Pokorny, A., Birkbeck, T.H., Almeida, P.F. (2002). Mechanism and kinetics of delta-lysin interaction with phospholipid vesicles. Biochem 41, 11044-11056.

Raingo, J., Khvotchev, M., Liu, P., Darios, F., Li, Y.C., Ramirez, D.M., Adachi, M., Lemieux, P., Toth, K., Davletov, B., Kavalali, E.T. (2012). VAMP4 directs synaptic vesicles to a pool that selectively maintains asynchrons neurotransmission. Nat Neurosci 15, 738-745.

Rao, T.C., Passmore, D.R., Peleman, A.R., Das, M., Chapman, E.R., Anantharam, A. (2014). Distinct fusion properties of synaptotagmin-1 and synaptotagmin-7 bearing dense core granules. Mol Biol Cell 25, 2416-2427.

Rawle, R.J., van Lengerich, B., Chung, M., Bendix, P.M., Boxer, S.G. (2011). Vesicle fusion observed by content transfer across a tethered lipid bilayer. Biophys J 18, 1093-1101.

Recktenwald, D.J., McConnell, H.M. (1981). Phase equilibria in binary mixtures of phosphatidylcholine and cholesterol. Biochem 20, 4505-4510.

Reim, K., Mansour, M., Varoqueaux, F., McMahon, H.T., Südhof, T.C., Brose, N., Rosenmund C. (2001). Complexins regulate a late step in Ca2-dependent neurotransmitter release. Cell 104, $71-81$. 
Roggero, C.M., De Blas, G.A., Dai, H., Tomes, C.N., Rizo, J., Mayorga, L.S. (2007).

Complexin/synaptotagmin interplay controls acrosomal exocytosis. J Biol Chem 282, 2633526343.

Rouser, G., Fkeischer, S., Yamamoto, A., (1970). Two dimensional then layer chromatographic separation of polar lipidsand determination of phospholipids by phosphorus analysis of spots. Lipids 5, 494-496.

Ryu, J.K., Jahn,R., Yoon, T.Y. (2016). Progresses in understanding N-ethylmaleimide sensitive factor mediated disassembly of SNARE complexes. Biopolymers 105, 518-531.

Ryu, J.K., Min, D., Rah, S.H., Kim, S.J., Park, Y., Kim, H., Hyeon, C., Kim, H.M., Jahn, R., Yoon, T.Y. (2015). Spring-loaded unraveling of a single SNAREcomplex by NSFinone roundofATP turnover. Science 347, 1485-1489.

Söllner, T.H. (2003). Regulated exocytosis and SNARE function. Mol Membr Biol 20, 209-220.

Südhof, T.C. (2013). Neurotransmitter release: The last millisecondin the life of synaptic vesicle. Neuron 80, 675-690.

Salaün, C., Gould, G.W., Chamberlain, L.H. (2005). Lipid raft association of SNARE proteins regulates exocytosis in PC12 cells. J Biol Chem 280, 19449-19453. 
Salaün, C., Gould, G.W. Chamberlain, L.H. (2005). The SNARE proteins SNAP-25 and SNAP23 display different affinitiesfor lipid rafts in PC12 cells. Regulation by distinct cysteine-rich domains. J Biol Chem 280,1236-1240.

Salzmann, M., Pervushin, K., Wider, G., Senn, H., Wüthrich, K. (1998). TROSY in tripleresonance experiments: new perspective for sequential NMR assignment of large proteins. Proc Natl Acad Sci 95, 13585-13590.

Sankaram, M.B., Thompson, T.E., (1990). Modulation of phopholipid acyl chain order by cholesterol. A solid-state 2H nuclear magnetic resonance study. Biochem 28, 10676-10684.

Scheuner, D., Holz, R.W. (1994). Evidence that the ability to respond to a calcium stimulus in exocytosis is determined by the secretory granule membrane: Comparison of exocytosis of injected bovine chromaffin granule membranes and endogenous cortical granules in xenopus laevis oocytes. Cell MolNeurobiol 14, 245-257.

Scheuner, D., Logsdon, C.D., Holz, R.W. (1992). Bovine chromaffin granule membranes undergo $\mathrm{Ca}(2+)$-regulated exocytosisin frogoocytes. J Cell Biol 116, 359-365.

Schoch, S., Deák, F., Königstorfer, A., Mozhayeva, M., Sara, Y., Südhof, T.C., Kavalali, E.T. (2001). SNARE function analyzed in synaptobrevin/VAMP knockout mice. Science 294, 11171122. 
Schuette, C.G., Hatsuzawa, K., Margittai, M., Stein, A., Riedel, D., Küster, P., König, M., Seidel, C., Jahn, R. (2004). Determinants of liposome fusion mediated by synaptic SNARE proteins. Proc Natl Acad Sci USA 101, 2858-2863.

Seiler, F., Malsam, J., Krause, J.M., Sollner, T.H. (2009). A role of complexin-lipid interactions in membrane fusion. FEBS Letters 583, 2343-2348.

Shen, J., Tareste, D.C., Paumet, F., Rothman, J.E., Melia, T.J. (2007). Selective activation of cognate SNAREpins by Sec1/Munc18 proteins. Cell 128, 183-195.

Shi, L., Shen, Q.T., Kiel, A., Wang, J., Wang, H.W., Melia, T.J. Rothman, J.E., Pincet, F. (2012). NARE proteins: One to fuse and three to keep the nascent fusion pore open. Science 335, 1355-1359.

Simons, K., Geri, M.J. (2010). Revitalizing membrane rafts: new tools and insights. Nat Rev Mol Cell Biol 11, 688-699.

Sinha, R., Ahmed, S., Jahn, R., Klingauf, J. (2011). Two synaptobrevin molecules are sufficient for vesicle fusion in central nervous system synapses. Proc Natl Acad Sci USA 108, 1431814323.

Smith, E.A., Weisshaar, J.C. (2011). Docking, not fusion, as the rate-limiting step in a SNARE driven vesicle fusion assay. Biophys J 100, 2141-2150. 
Snead, D., Wragg, R.T., Dittman, J.S., Eliezer, D. (2014). Membrane curvature sensing by the Cterminal domain of complexin. Nat Comm 5, 4955.

Sorensen, J.B. (2004). Formation, stabilization, and fusion of the readily releaseable pool of secretory vesicles. Pflugers Arch 448, 347-362.

Sorensen, J.B. (2009). Conflicting views on the membrane fusion machinery and thefusion pore. Annu Rev Cell Dev Biol 25, 513-537.

Stein, A., Radhakrishnan, A., Riedel, D., Fasshauer, D., Jahn, R. (2007). Synaptotagmin activates membrane fusion through a $\mathrm{Ca} 2+$-dependent trans interaction with phospholipids Nat Struct Mol Biol 14, 904-911.

Stein, A., Weber, G., Wahl, M.C., Jahn, R. (2009). Helical extension of the neuronal SNARE complex into the membrane. Nature 460, 525-528.

Südhof, T.C. (2014). The molecular machinery of neurotransmitter release (Nobel Lecture). Angew Chem Int Ed Engl 53, 12696-12717.

Südhof, T.C., Rothman, J.E. (2009). Membrane fusion: grappling with SNARE and SM proteins. Science 323, 474-477. 
Sutton, R.B., Fasshauer, D., Jahn, R., Brunger, A.T. (1998). Crystal structure of a SNARE complex involved in synaptic exocytosis at 2.4 A resolution. Nature 395, 347-353.

Tadokoro, S., Nakanishi, M., Hirashima, N. (2005). Complexin II Facilitates exocytotic release in mast cells by enhancing Ca2+ sensitivity of the fusion process. J Cell Sci 118, 2239-2246.

Takamori S, Holt M, Stenius K, Lemke EA, Grønborg M, Riedel D, Urlaub H, Schenck S, Brügger B, Ringler P, Müller SA, Rammner B, Gräter F, Hub JS, De Groot BL, Mieskes G, Moriyama Y, Klingauf J, Grubmüller H, Heuser J, Wieland F, Jahn R. (2006). Molecular anatomy of a trafficking organelle. Cell 127, 831-846.

Tamm, L.K., Crane, J., Kiessling, V. (2003). Membrane fusion: a structural perspective on the interplay of lipids and proteins. Curr Opin Struct Biol 13, 453-466.

Tang, J., Maximov, A., Shin, O.H., Dai, H., Rizo, J., Südhof, T.C. (2006). A complexin/synaptotagmin 1 switch controls fast synaptic vesicle exocytosis. Cell 126, 11751187.

Taraska, J.W., Perrais, D., Ohara-Imaizumi, M., Nagamatsu, S., Almers, W. (2003). Secretory granles are recaptured largely intact after stimulated exocytosis in cultured endocrine cells. Proc Natl Acad Sci USA 100, 2070-2075. 
Taverna, E., Saba, E., Rowe, J., Francolini, M., Clementi, F., Rosa, P. (2004). The role of lipid microdomains in $\mathrm{P} / \mathrm{Q}$-type calcium channel (Cav2.1) clustering and function in presynaptic membranes. J Bio Chem 279, 5127-5134.

Thiele, C., Hannah, M.J., Fahrenholz, F., Huttner, W.B. (2000). Cholesterol binds to synaptophysin and is required for biogenesis of synaptic vesicles. Nat Cell Biol 2, 42-49.

Thorn, P., Zorec, R., Rettig, J., Keating, D.J. (2016). Exocytosis in non-neuronal cells. J Neurochem 137, 849-859.

Tong, J., Borbat, P.P., Freed, J.H., Shin, Y.K. (2009). A scissors mechanism for stimulation of SNARE-mediated lipidmixing by cholesterol. Proc Natl Acad Sci USA 106, 5141-5146.

Tooner, R.F., Verhage, M. (2007). Munc18-1 in secretion: lonely munc joins SNARE team and takes control. Trends Neurosci 40, 564-572.

Trimbuch, T., Xu, J., Flaherty, D., Tomchick, D.R., Rizo, J., Rosenmund, C. (2014). Reexamining how complexin inhibits neurotransmitter release. Elife 3, e02391.

van den Bogaart, G., Holt, M.G., Bunt, G., Riedel, D., Wouters, F.S., Jahn, R. (2010). One SNARE complex is sufficient for membrane fusion. Nat Struct Mol Biol 17, 358-364. 
van den Bogaart, G., Lang, T., Jahn, R., (2013). Microdoamins of SNARE proteins in the plasma membrane. Curr Top Membr 72, 193-230.

van den Bogaart, G., Meyenberg, K., Risselada, H.J., Amin, H., Willig, K.I., Hubrich, B.E., Dier, M., Hell, S.W., Grubmüller, H., Diederichsen, U., Jahn, R. (2011). Membrane protein sequestering by ionic protein-lipid interactions. Nature 479, 552-555.

van den Ent, F., Lowe, J. (2006). RF cloning: A restriction-free methods forinserting target genesintoplasmids.J Biochem Biophys Methods 67, 67-74.

van den Hoff, M.J., Moorman, A.F., Lamers, W.H. (1992). Electroporation in intracellular buffer increases cell survival. Nucleic Acids Res 20, 2902.

van Meer, G., Voelker, D.R., Feigenson, G.W. (2008). Membrane lipids: where they are and how they behave. Nat Rev Mol Cell Biol 9, 112-124.

Vardjahn, N., Jorgacevski, J., Zorec, R. (2013) Neuroscientist 19, 160-174.

Varoqueaux, F., Sigler, A., Rhee, J.S., Brose, N., Enk, C., Reim, K., Rosenmund, C. (2002). Total arrest of spontaneous and evoked synaptic transmission but normal synaptogenesis in the absence of Munc13-mediated vesicle priming. Proc Natl Acad Sci USA 99, 9037-9042.

Verhage, M., Maia, A.S., Plomp, J.J., Brussaard, A.B., Heeroma, J.H., Vermeer, H., Toonen, R.F., Hammer, R.E., van den Berg, T.K., Missler, M., Geuze, H.J., Südhof, T.C. (2000). 
Synaptic assembly of the brain in the absence of neurotransmitter secretion. Science 287, 864869.

Victor, K.G., Cafiso, D.S. (2001). Location and dynamics of basic peptides at the membrane interface: electron paramagnetic resonance spectrosocopy of tetramethyl-piperidine-N-oxyl-4amino-4-carboxylic acid-labeled peptides. Biophys J 81, 2241-2250.

Viet, M., Söllner, T.H., Rothman, J.E. (1996). Multiple palmitoylation of synaptotagmin and the t-SNARE SNAP-25. FEBS Lett 385, 119-123.

Wagner, M.L., Tamm, L.K. (2001). Reconstituted syntaxin-1a/SNAP-25 interacts with negatively charged lipids as measured by lateral diffusion by lateral diffusion in planar supported bilayers. Biophys J 81, 266-275.

Walter, A.M., Kurps, J., de Wit, H., Schöning, S., Toft-Bertelsen, T.L., Lauks, J., Ziomkiewicz, I., Weiss, A.N., Schulz, A., Fischer von Mollard, G., Verhage, M., Sorensen, J.B. (2014). The SNARE protein vtila functions in dense-core vesicle biogenesis. EMBO J 33, 1681-1697.

Wan, C., Kiessling, V., Cafiso, D.S., Tamm, L.K. (2011). Partitioning of synaptotagmin I C2 domains between liquid-ordered and liquid-disordered inner leaflet lipid phases. Biochem 50, $2478-2485$. 
Wang, L., Seeley, E.S., Wickner, W., Merz, A.J. (2002). Vacuaole fusion at a ring of vertex docking sites leaves membrane fragments within the organelle. Cell 108, 357-369.

Wang, T., Smith, E.A., Chapman, E.R., Weisshaar, J.C. (2009). Lipid mixing and content release in single-vesicle, SNARE driven fusion assay with 1-5 ms resolution. Biophys J 96, 4122-4131.

Wang, W., Yang, L., Huang, H.W. (2007). Evidence of cholesterol accumulated in high curvature regions: implication to the curvature elastic energy for lipid mixtures. Biophys J 92, 2819-2830.

Wang, Z., Liu, H., Gu, Y., Chapman, E.R. (2011). Reconstituted synaptotagmin I mediates vesicle docking, priming, and fusion. J Cell Biol 195, 1159-1170.

Wasser, C.R., Ertunc, M., Liu, X., Kavalali, E.T. (2007). Cholesterol-dependent balance between evoked and spontaneous synaptic vesicle recycling. J Physiol 579, 413-429.

Weber, T., Zemelman, B.V., McNew, J.A., Westermann, B., Gmachl, M., Parlati, F., Söllner, T.H., Rothman, J.E. (1998). SNAREpins: minimal machinery for membrane fusion. Cell 92, 759-772.

Weninger, K., Bowen, M.E., Choi, U.B., Chu, S., Brunger, A.T. (2008). Accessory proteins stabilize the acceptor complex for synaptobrevin, the 1:1 syntaxin/SNAP-25 complex. Structure $16,308-320$. 
Wickner, W., Schekman, R. (2008). Membrane fusion. Nat Struct Mol Biol 15, 658-664.

Widerhold, K., Fasshauer, D. (2009). Is assembly of the SNARE complex enough to fule membrane fusion? J Biol Chem 284, 13143-13152.

Wragg, R.T., Snead, D., Dong, Y., Ramlall, T.F., Menon, I., Bai, J., Eliezer, D., Dittman, J.S. (2013). Synaptic vesicles position complexin to block spontaneous fusion. Neuron 77, 323-334.

Xiao, W., Poirier, M.A., Bennett, M.K., Shin, Y.K. (2001). The neuronal t-SNARE complex is a parallel four-helix bundle. Nat Struct Biol 8, 308-311.

Xue, M., Reim, K., Chen, X., Chao, H.T., Deng, H., Rizo, J., Brose, N., Rosenmund, C. (2007). Distinct domains of complexin I differentially regulate neurotransmitter release. Nat Struct Mol Biol 14, 949-958.

Xu, X.M., Yoo, M.H., Carlson, B.A., Gladyshev, V.N., Hatfield, D.L. (2009). Simultaneous knockdown of the expression of two genes using multiple shRNAs and subsequent knock-in of their expression. Nat Protoc 4, 1338-1348.

Yang, L., Huang, H.W. (2002). Observation of a membrane fusion intermediate structure. Science 297, 1877-1879. 
Yang, S.T., Kreutzberger, A.J., Lee, J., Kiessling, V., Tamm, L.K. (2016). The role of cholesterol in membrane fusion. Chem Phys Lipids 199, 136-143.

Yang, X., Cao, P., Südhof, T.C. (2013). Deconstructing complexin function inactivating and clamping Ca2+-triggered exocytosis by comparing knockout and knockdown phenotypes. Proc Natl Acad Sci USA 110, 20777-20782.

Yang, X., Kaeser-Woo, Y.J., Pang, Z.P., Xu, W., Südhof, T.C. (2010). Complexin clamps asynchronous release by blocking a secondary $\mathrm{Ca}(2+)$ sensor via its accessory alpha helix. Neuron 68, 907-920.

Yeagle, P.L. (1985). Lanosterol and cholesterol have different effects on phospholipid acyl chain ordering. Biochim Biophys Acta 815, 33-36.

Yoon, T.Y., Lu, X., Diao, J., Lee, S.M., Ha, T., Shin, Y.K. (2008). Complexin and Ca2+ stimulated SNARE-mediated membrane fusion. Nat Struct Mol Biol 15, 707-713.

Zamir, O., Charlton, M.P. (2006). Cholesterol and synaptic transmitter release at crayfish neuromuscular junctions. J Physiol 571, 83-99.

Zareh, S.K., DeSantis, M.C., Kessler, J.M., Li, J.L., Wang, Y.M. (2012). Single-image diffusion coefficient measurements of proteins in free solution. Biophys J 102, 1685-1691. 
Zbili, M., Rama, S., Debanne D. (2016). Dynamic control of neurotransmitter release by presynaptic potential. Front Cell Neurosci 10, 278.

Zdanowicz R, Kreutzberger, A.J., Liang, B., Kiessling, V., Tamm, L.K., Cafiso, D.S. (2017). Simultaneous complexin binding to acceptor t-SNARE complex and membranes explains its clamping effect on v-SNARE insertion and fusion. Biophys J, in press.

Zenisek, D., Steyer, J.A., Almers, W. (2000). Transport, capture, and exocytosis of single synaptic vesicles at active zones. Nature 406, 849-854.

Zhang, J., Xue, R., Ong, W.Y., Chen, P. (2009). Roles of cholesterol in vesicle fusion and motion. Biophys J 22, 433-466.

Zhang, Z., Wu, Y., Wang, Z., Dunning, F.M., Rehfuss, J., Ramanan, D., Chapman, E.R., Jackson, M.B. (2011). Release mode of large and sall dense-core vesicles specified by different synaptotagmin isoforms in PC12 cells. Mol Biol Cell 22, 2324-2336.

Zhao, M., Wu, S., Zhou, Q., Vivona, S., Cipriano, D.J., Cheng, Y., Brunger, A.T. (2015). Mechanistic insights into the recycling machine of the SNARE complex. Nature 518, 61-67.

Zheng, L., Baumann, U., Reymond, J.L. (2004). An efficient one-step site-directed and sitesaturation mutagenesis protocol. Nucleic Acids Res 32, e115. 